Site-Specific Propagation Prediction for Wireless

In-Building Personal Communication System Design

$$
\text { by }
$$

Scott Y. Seidel

Dissertation submitted to the Faculty of the

Virginia Polytechnic Institute and State University

in partial fulfillment of the requirements for the degree of

Doctor of Philosophy

in

Electrical Engineering

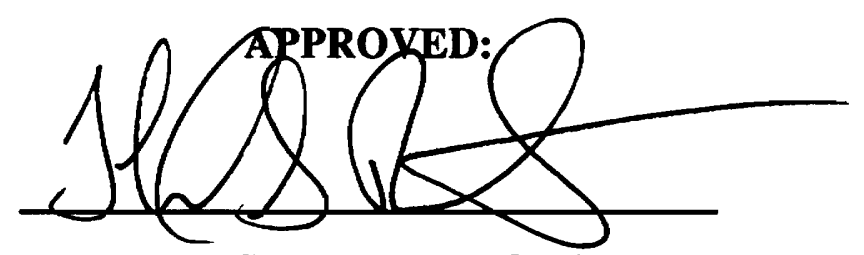

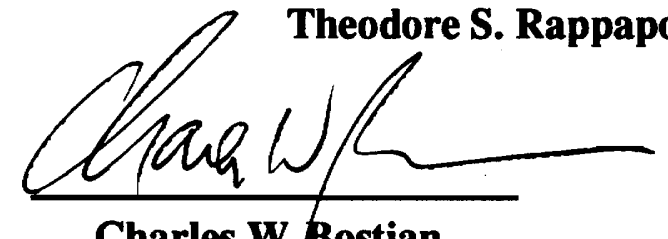

Charles W. Bostian

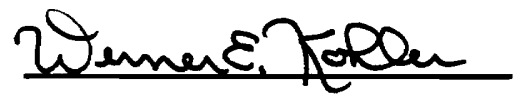

Werner E. Kohler

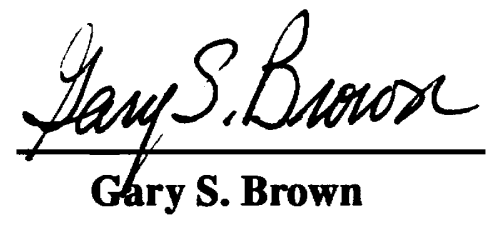

Bricen P. Noermer

Brian D. Woerner

February 7, 1993 
c. 2

5452 


\title{
Site-Specific Propagation Prediction for Wireless In-Building Personal Communication System Design
}

\author{
by \\ Scott Yates Seidel \\ Theodore S. Rappaport, chairman \\ Electrical Engineering \\ (ABSTRACT)
}

This dissertation describes a geometrical optics model to predict propagation in buildings for personal communication system (PCS) design.Background on the growth of wireless communications is given, and the importance of accurate propagation models is discussed. The peculiarities of propagation in mobile and portable radio environments, particularly multipath propagation and its effects on transmitted signals, are described. Current in-building propagation models are presented and the progression from statistical models to site-specific models is outlined. Theoretical models for radiowave propagation in the presence of scattering bodies are merged with a site-specific description of the propagation environment to improve upon the accuracy of existing propagation models.

A geometrical optics ray tracing model for predicting propagation based on a building blueprint representation is developed for a transmitter and receiver located on the same floor inside a building. The measured and predicted propagation data are presented as power delay profiles that contain the amplitude and arrival time of individual multipath components. Measured and predicted power delay profiles are compared on a location-bylocation basis to provide both a qualitative and a quantitative measure of the model accuracy. The concept of effective building material properties is developed and the effective building material properties are derived for two dissimilar buildings based upon comparing measured and predicted power delay profiles. Time delay comparison shows that the amplitudes of many significant multipath components are accurately predicted by this model. Path loss between a transmitter and receiver is predicted-with a standard deviation of less than $5 \mathrm{~dB}$. Ideas for improving the accuracy and expanding the applicability of the models applied here to wireless in-building propagation prediction are suggested. 


\section{Foreword}

This work has developed in response to the growth of wireless communications systems. The success of cellular radio indicates that ubiquitous personal communications can become a reality. As part of a complete Personal Communications System (PCS), wireless coverage will be required inside buildings. The propagation conditions severely affect the quality of communications over a radio link. These propagation conditions are highly dependent upon the location of major objects such as walls and partitions inside buildings.

To date, in-building propagation models have incorporated only a limited amount of site-specific information concerning the propagation environment. Yet, it is the specific geometry that influences the propagation. Hence, the major contribution of this dissertation is that the propagation characteristics may be accurately predicted by incorporating information about the location, size, and electrical properties of major building features. Two different contributions to site-specific propagation prediction are presented. First, a soft-partition and concrete wall attenuation factor path loss model for a single floor system is given. Also, a floor attenuation factor path loss model for multi-floored office buildings that contain large open areas where individual offices are cubicles separated by cloth-covered soft partitions is presented.

The second model is a more general ray tracing method that can be used to predict power delay profiles for transmitters and receivers located on the same floor within a building. The power delay profile contains both path loss and time delay information. Innovative contributions to ray tracing include a novel way to determine the source ray directions in three dimensions, the development of a partially automated ray tracing computer code that includes the use of a standard computer aided design (CAD) program for the building database, and an optimization routine for determining effective building material electrical properties. This work presents the first ray tracing for propagation prediction where measured and predicted power delay profiles are compared on a location-by-location basis as a function of time delay. An error curve as a function of excess delay is defined, and the area under the squared error curve is minimized by varying the reflection 
coefficients of the building materials. Important propagation channel statistics that include path loss, rms delay spread, and delay interval are compared on a location-by-location basis to quantify the model accuracy.

A thorough literature review is included throughout this work. First, a background for understanding propagation in mobile and portable radio environments is discussed. Measurement-based propagation models are compiled from the literature. These works start from statistical descriptions of how fast mean path loss increases with distance and median and maximum rms delay spread, and lead into site-specific path loss models in multi-floored and soft-partitioned environments developed by the author. In order to develop more accurate propagation models, electromagnetic theory concerning the interaction of radio waves with scattering objects is presented. This includes the theory of geometrical optics, implemented by the automated propagation prediction tool.

The chapter on scattering covers fundamental aspects of a propagation mechanism that are often misunderstood. A description of physical optics and the geometrical theory of diffraction are given, and these electromagnetics models are applied to the scattering from a smooth rectangular flat plate. These models can be used to compute the scattering from interior and exterior building walls in either an indoor or an outdoor microcellular radio environment. These models are compared and contrasted with a heuristic model for scattering from similar surfaces given in [Sch92]. Limitations for the applicability of each model in a site-specific propagation prediction tool are given.

This dissertation presents a thorough background of the mobile and portable radio propagation environment. Using knowledge of this environment, theoretical electromagnetic scattering models are merged with site-specific information about the physical propagation environment to predict the propagation channel characteristics as a function of location. This represents a major shift in the development and application of propagation models. Previously developed models for propagation in buildings have only incorporated limited amounts of site-specific information, and these models then rely on statistical results of radio propagation measurements. With the advent of increased computational power and more efficient coding algorithms, the models developed in this dissertation can 
be used to rapidly design and install wireless personal communications systems in a wide variety of buildings. 


\section{Acknowledgments}

My sincere appreciation goes to my academic advisor, Professor Theodore S. Rappaport. His guidance and inspiration have provided an invaluable experience during my tenure as a research associate with the Mobile and Portable Radio Research Group (MPRG). I am continually amazed by his enthusiasm and dedication to wireless personal communications. I have been honored to have Charles Bostian, Gary Brown, Werner Kohler and Brian Woerner as members of my graduate committee. Their support has been exceptional, and I extend my appreciation to each of them.

This work was supported in part by a National Science Foundation Graduate Research Fellowship. I am also indebted to the MPRG Industrial Affiliates program for providing the funding for the research group as a whole. Without this funding, it would not have been possible to measure in-building propagation or implement the prediction models on the MPRG network of SUN SparcStations.

The support of the research staff, Linda Solowiej, Prab Koushik, and Mike Keitz have proven helpful in many situations. I wish to thank my friends in the research group for providing the support and encouragement which I very much needed at times during the course of this research. A special thanks are to those persons whom I have been fortunate enough to share an office with, Dwayne Hawbaker, Joe Liberti, Kurt Schaubach, Ken Blackard, Carl Dietrich, and Peter Ho. The informal discussions relating to research and other topics are an unforgettable ingredient in the research experience.

I wish to acknowledge the support, encouragement, and love of my wife Debbie. Her support has been incredible. I do not know how I could have completed this work without her help. I also wish to give a special thank you to my parents for raising me to be who I am. Above all, I give thanks and glory to God for blessing me with the many gifts given me. I truly feel blessed to have had the opportunity for this experience. 


\section{Table of Contents}

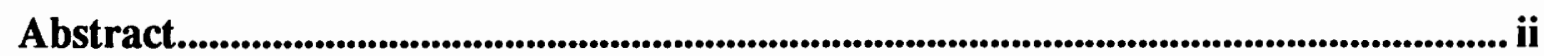

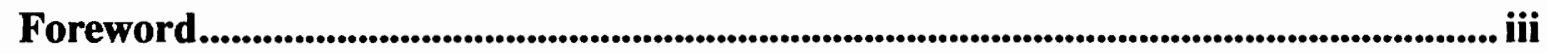

Acknowledgements ............................................................................................................... vi

Table of Contents ............................................................................................................... vii

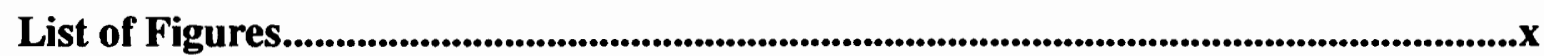

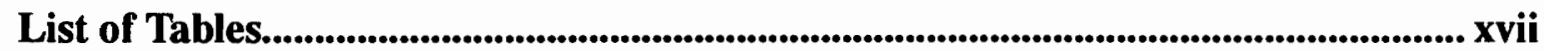

1 Introduction .................................................................................................................

1.1 The Growth of Wireless Communications in Recent Years...............................

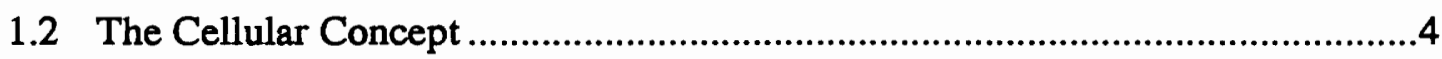

1.3 The Importance of Accurate Propagation Models ..............................................5

2 The Mobile and Portable Radio Propagation Environment ....................................8

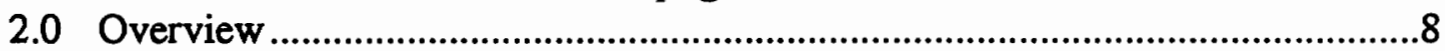

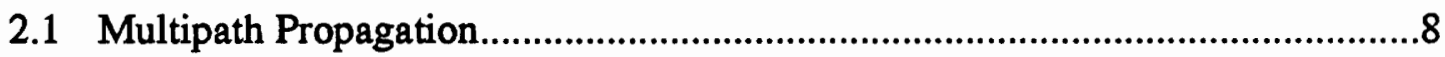

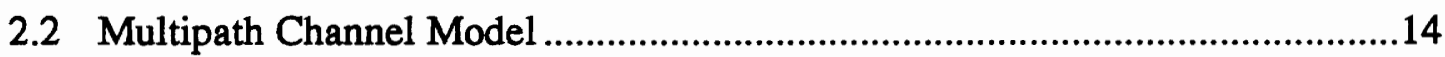

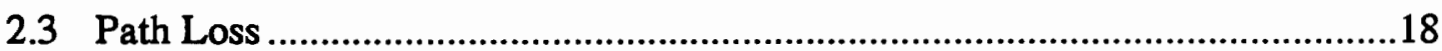

2.3.1 Computing Path Loss ..................................................................18

2.3.2 Statistical Mean Path Loss Exponent..................................................18

2.4 Time-Dispersive Multipath Channel Parameters ...............................................

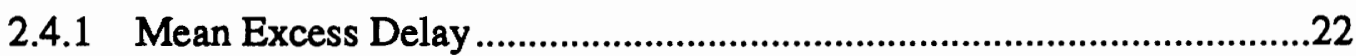

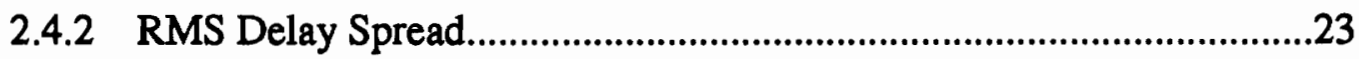

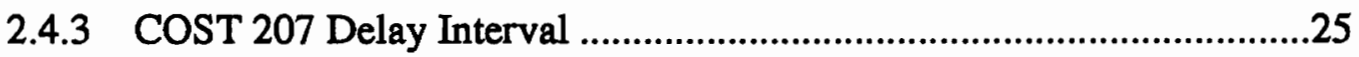

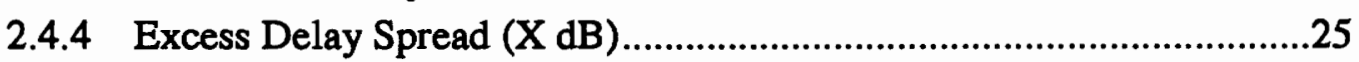

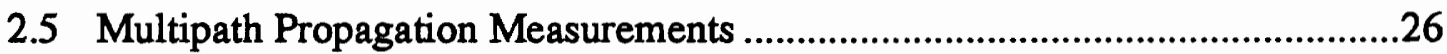

2.5.1 CW Measurements and Data Processing ..........................................27

2.5.2 Direct Pulse Measurements and Data Processing ..................................29

2.5.3 Spread Spectrum Measurements ..........................................................33

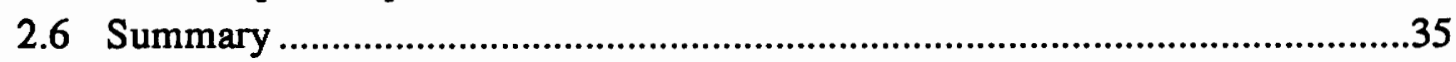

3 Propagation Models Based on Measurements ......................................................37

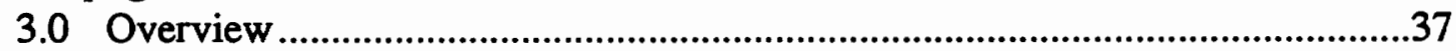

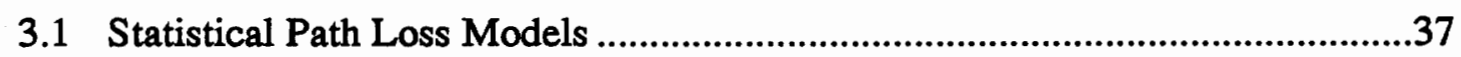

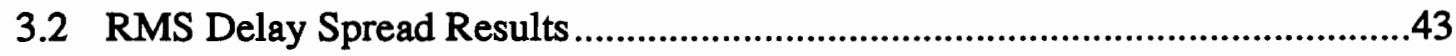

3.3 The Effects of Carrier Frequency on Propagation Characteristics.....................45

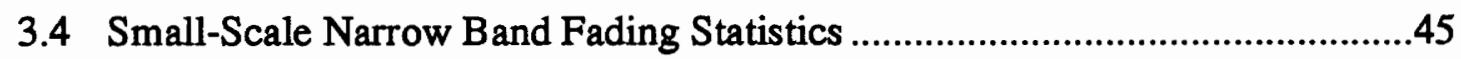

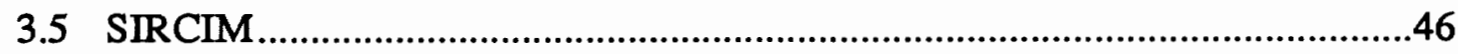


3.6 Site-Specific Path Loss Models......................................................................48

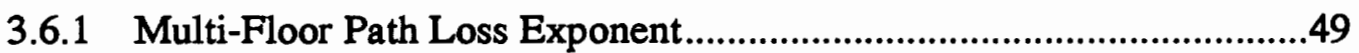

3.6.2 Floor Attenuation Factor (FAF) Path Loss Model ..............................50

3.6.3 Application of Path Loss Models in Multi-Floored Environments.......52

3.6.4 Path Loss Contour Plots ...............................................................53

3.6.5 Soft Partition and Concrete Wall Attenuation Factor Model .................54

3.6.6 Predicted Path Loss for Soft Partitioned Environments.........................57

3.6.7 Shadowing Caused by Typical Objects Found in Buildings.................59

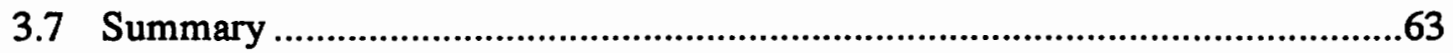

4 Geometrical Optics, Scattering, and Diffraction ..................................................64

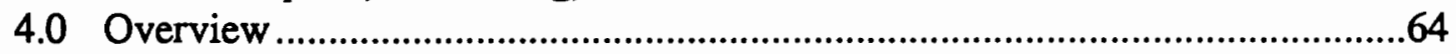

4.1 Electromagnetic Scattering by Arbitrarily Shaped Bodies ..............................64

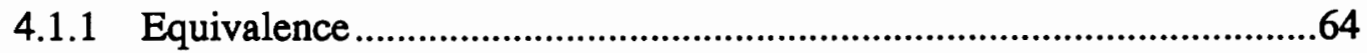

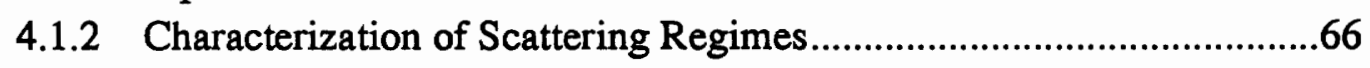

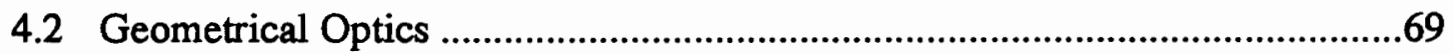

4.2.1 Properties of the Geometrical Optics Fields ......................................72

4.2.2 Free Space Path Loss ..........................................................................73

4.2.3 Plane Wave Obliquely Incident Upon a Planar Boundary ....................76

4.2.4 Plane-Earth Reflection Path Loss Model ..........................................82

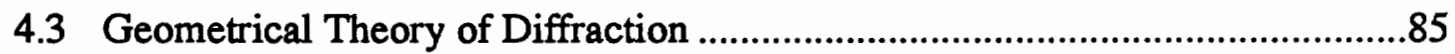

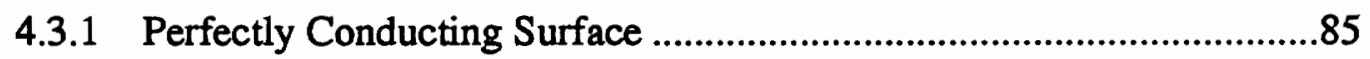

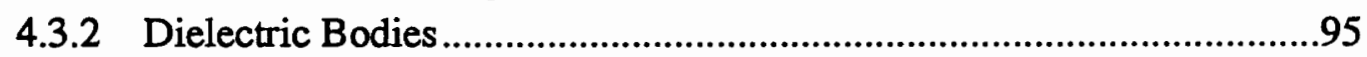

4.3.3 Application of Uniform GTD to a Perfectly Conducting Flat

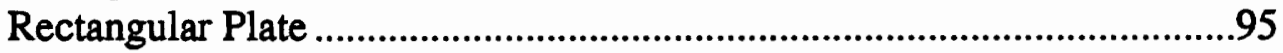

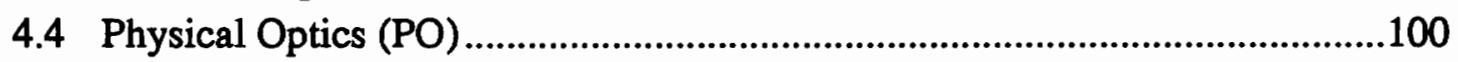

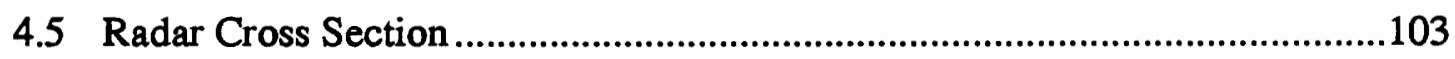

4.5.1 The Bistatic Radar Equation in Multipath Environments...................103

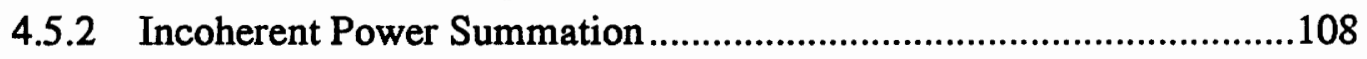

4.6 Computed Scattering and Diffraction Patterns ............................................111

4.6.1 Numerical Comparison of Scattering and Diffraction Models ...........111

4.6.2 Implementation and Complexity Issues..........................................117

4.6.3 Scattering Patterns as a Function of Different Parameters...................118

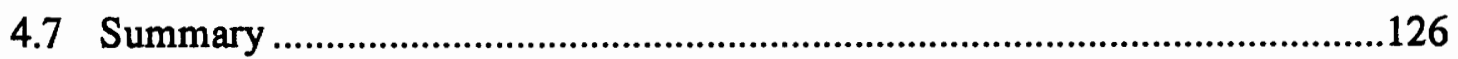

5 Site-Specific In-Building Propagation Prediction.....................................................129

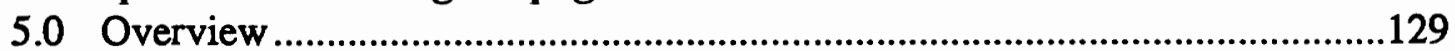

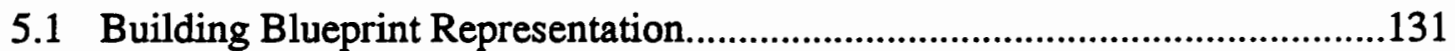

5.1.1 Conversion of AutoCAD Data Format to Ray Tracing Input .............131

5.1.2 Transmitter and Receiver Locations .............................................134 
5.1.3 Ray Tracing Input File (.ray) .........................................................134

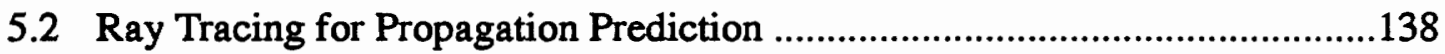

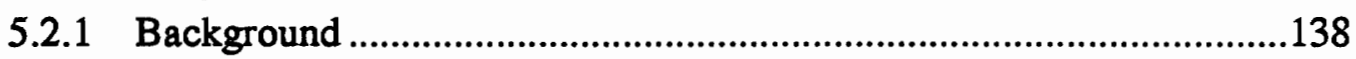

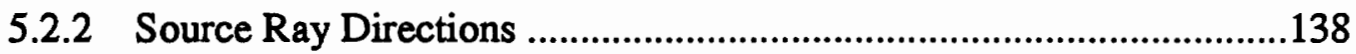

5.2.3 Tracing The Rays at a Boundary ..................................................142

5.2.4 Representation of Propagation Models in the Ray Tracing Program .144

5.2.5 Identification of Specular Rays .........................................................147

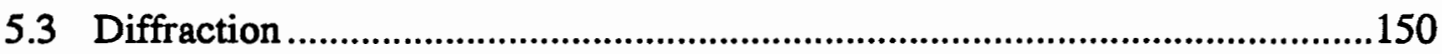

5.4 Processing Raw Ray Tracing Program Output ..............................................151

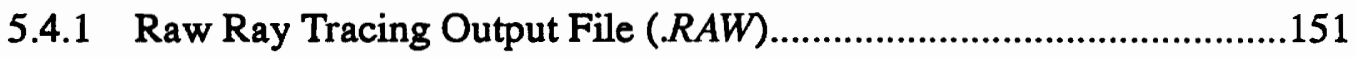

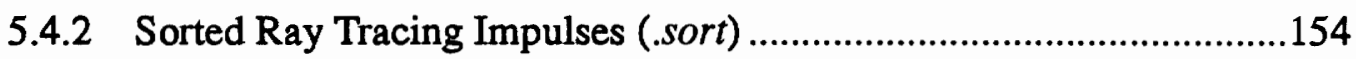

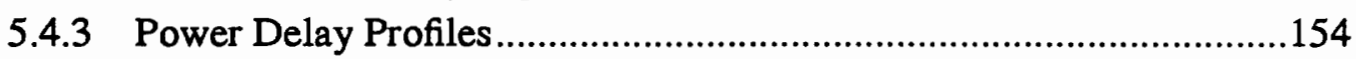

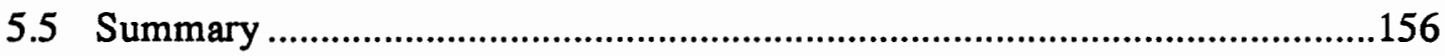

6 Comparison of Measured and Predicted Propagation...............................................157

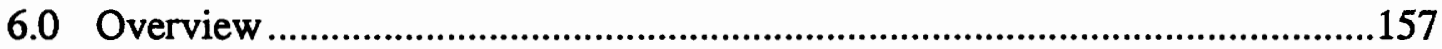

6.1 Comparison Criteria and Error Function Definition .....................................159

6.2 Optimization of Effective Building Material Properties ....................................161

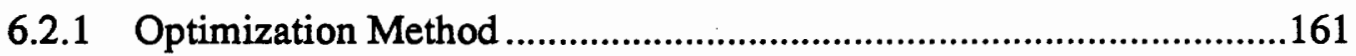

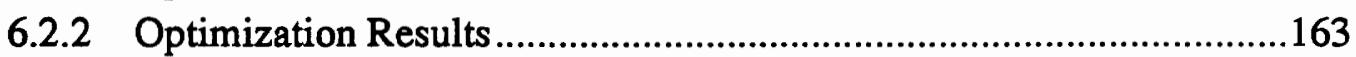

6.2.3 Analysis of Optimized Error Function With Excess Delay .................164

6.3 Comparison of Measured and Predicted Power Delay Profiles .......................166

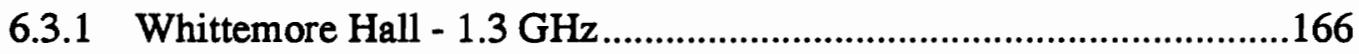

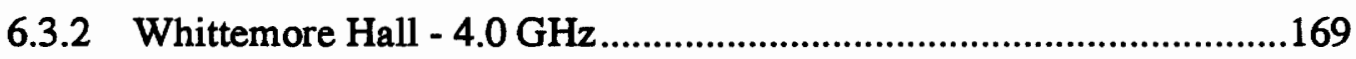

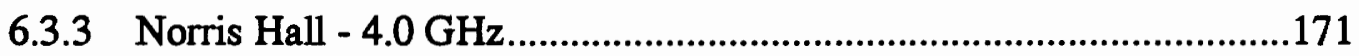

6.4 Comparison of Measured and Predicted Channel Parameters ........................175

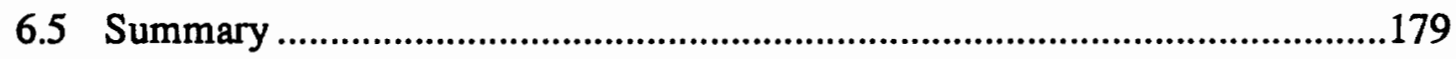

7 Conclusions and Future Work ......................................................................182

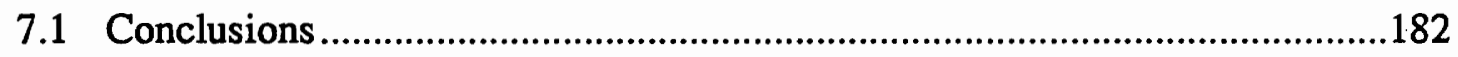

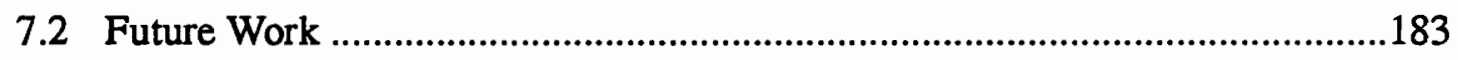

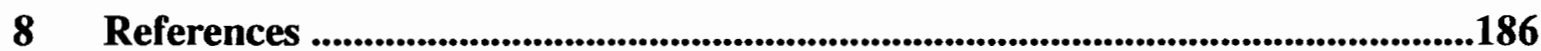

APPENDIX A: Fresnel Plane Wave Reflection Coefficients ................................197

APPENDIX B: Physical Optics ......................................................................202

APPENDIX C: Measured and Predicted Power Delay Profiles...........................206

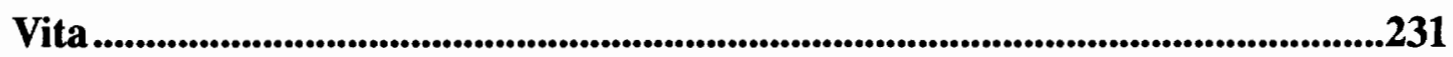




\section{List of Figures}

FIGURE 1.2-1. A seven frequency re-use pattern used in cellular radio. The same frequencies are used in all cells marked with the letter ' $A$ '.

FIGURE 2.1-1. A transmitter and a receiver located in a multipath scattering environment (from [Dev87c]).

FIGURE 2.1-2. The effects of multipath propagation on the received signal for different types of transmitted signals (from [Dev87c]).

FIGURE 2.1-3. Multipath channel impulse response and frequency spectrum.

FIGURE 2.1-4. Complex envelope of a pulsed RF signal. The complex envelope is the low-pass representation of the amplitude variations on an RF carrier.

FIGURE 2.1-5. Impulse response estimate for various probing bandwidths. Part a.) shows a perfect impulse response, and parts b.), c.), and d.) show the effect of decreasing bandwidth on time resolution.

FIGURE 2.3-1. Example of CW fading. The local mean averaged over $20 \lambda$ is also shown.

FIGURE 2.4-1. Measured power delay profile and statistical parameters used to describe the measurements.

FIGURE 2.4-2. Measured power delay profile and COST 207 delay interval for $90 \%$ of the received power.

FIGURE 2.4-3. Measured power delay profile and excess delay $10 \mathrm{~dB}$......................26

FIGURE 2.5-1. Block diagram of a CW measurement system.................................28

FIGURE 2.5-2. Typical CW fading run. The moving average over a $20 \lambda$ distance is also shown in the figure.

FIGURE 2.5-3. Block diagram of a typical pulsed transmitter.

FIGURE 2.5-4. Block diagram of a typical wide band direct pulse receiver. The receiver is a tuned $R F$ receiver.

FIGURE 2.5-5. Continuous spread spectrum transmitter.............................................34

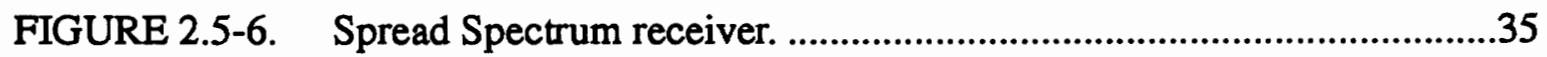

FIGURE 3.1-1. Scatter plot of CW path loss as a function of distance in office building 1 . The symbols indicate the number of floors between the transmitter and receiver (from [Sei92a]).

FIGURE 3.1-2. Scatter plot of CW path loss as a function of distance in office building 2. Notice the clustering of data as a function of the number of floors between transmitter and receiver (from [Sei92a]).

FIGURE 3.6-1. Contour plot of measured path loss for the fifth floor of office building 1 in [Sei92a].

FIGURE 3.6-2. Contour plot of measured path loss for the fourth floor of office building 1 in [Sei92a] 
FIGURE 3.6-3. Scatter plot of measured and predicted path loss in soft-partitioned environments as measured and predicted in [Sei92a]. The results were optimized over the combination of measurement locations in all three buildings.

FIGURE 3.6-4. Error contour plot of predicted path loss for the soft partitioned West wing of the 5 th floor of office building 1 in [Sei92a].

FIGURE 3.6-5. Error contour plot for the soft partitioned West wing on the 4th floor of office building 1 in [Sei92a].

FIGURE 4.1-1. An electromagnetic wave in the presence of an arbitrarily shaped scatterer.

FIGURE 4.1-2. Equivalent problem in the exterior region for an electromagnetic wave in the presence of an arbitrarily shaped scatterer.

FIGURE 4.1-3. Shadow zone for a scattering object.

FIGURE 4.1-4. Concave scatterer that induces multiple scattering from a single scattering object.

FIGURE 4.2-1. A tube of rays diverging from a point source.

FIGURE 4.2-2. Transmitter and receiver antenna located in free space (from [Col85]). . 73

FIGURE 4.2-3. Reflection and transmission field polarization of a perpendicularly (horizontally) polarized plane wave obliquely incident upon a planar dielectric boundary. Note that the entire region below the boundary is made up of the dielectric material and that the interface extends infinitely in the positive and negative $z$ direction.

FIGURE 4.2-4. Reflection and transmission field polarization of a vertically polarized plane wave incident upon a planar dielectric boundary. Note that the entire surface below the interface is made up of the dielectric medium and that the surface boundary extends to positive and negative infinity in the $\mathrm{z}$ direction.

FIGURE 4.2-5. Magnitude of the reflection and transmission coefficient of plicene cement and marble for both vertical and horizontal polarization at 4.0 $\mathrm{GHz}$. The dielectric properties are found in [Von54].

FIGURE 4.2-6. Pictoral representation of plane-Earth reflection.

FIGURE 4.2-7. Received signal as the receiver is moved radially away from the transmitter. Received power decreases with distance at a rate of $10 n$ $\mathrm{dB} /$ decade.

FIGURE 4.3-1. Canonical problem of diffraction by a perfectly conducting wedge of interior angle (2-n) $\pi$ (from [Bal89]). The angles are indicated in Figure 4.3-2. The points $Q_{R}$ and $Q_{D}$ are the points of reflection and diffraction on the wedge. 
FIGURE 4.3-2. Wedge geometry for a perfectly conducting wedge. The source is a distance $\rho^{\prime}=r_{1}$ from the tip of the wedge, and the observation point is at a distance $\rho=r_{2}$ from the tip of the wedge.

FIGURE 4.3-3. Field distribution of various components of a soft polarized plane wave incident normally on a conducting half-plane (from [Bal89]). The geometrical optics field, the incident diffracted field, and the reflected diffracted field are shown along with the total field which is the phasor sum of the geometrical optics, incident diffracted, and reflected diffracted fields. The observation angle is the angle of the receiver as measured from the face of the wedge as indicated in Figure 4.3-2.

FIGURE 4.3-4. Geometry for scattering from an infinite strip of finite width $b$ .93

FIGURE 4.3-5. Individual UTD field components scattered from an infinitely long finite width strip shown in Figure 4.3-4.

FIGURE 4.3-6. GO, GTD, and UTD diffraction solutions for scattering from a finite width strip. Notice that the Keller GTD solution is singular at the shadow boundaries.

FIGURE 4.3-7. Two-dimensional view of diffraction geometry for scattering from an infinite length strip of finite width $b$.

FIGURE 4.3-8. Transmitter and image source for scattering from a flat plate. The specular point is the intersection of line $A D$ and a straight line drawn between the receiver and the image source. The specular point lies on the surface if the intersection point is between points $B$ and $C$

FIGURE 4.4-1. Geometry for scattering by a perfectly conducting flat plate with area $a b$. An electromagnetic wave is incident at an angle $\theta_{\mathrm{i}}$ from the surface normal, and $\phi_{i}$ from the $x$-axis as indicated in the figure. .....101

FIGURE 4.4-2. Incident and scattered field polarization of a perpendicularly (TE) polarized plane wave incident upon a finite flat plate scatterer $\left(\phi_{i}=270\right.$, $\phi_{\mathrm{s}}=90$ ).

FIGURE 4.5-1. Bistatic radar used to detect the presence of aircraft. 105

FIGURE 4.5-2. Scatter plot of observed radar cross-sections of buildings. 107

FIGURE 4.5-3. Geometry for non-coherent 'Mini-RCS' scattering model (from [Sch92].

FIGURE 4.5-4 Incident and scattering angles for the two non-coherent scattering models in equations (4.5-2) and (4.5-3).

FIGURE 4.6-1. Assumed geometry for computing scattering patterns in the principle plane as a function of scattering angle from the surface normal. ......114

FIGURE 4.6-2. Scattered power as a function of angle with respect to the surface normal for four different scattering models. Both the transmitter and receiver are in the far-field of the scatterer. 
FIGURE 4.6-3. Scattered power as a function of angle with respect to the surface normal for four different scattering models. Both the transmitter and receiver are an order of magnitude closer to the scatterer than in Figure 4.6-2.

FIGURE 4.6-4. Scattered power as a function of angle with respect to the surface normal for four different scattering models. Both the transmitter and receiver are in the near field of the scatterer.

FIGURE 4.6-5. Backscattering as a function of incidence and scattering angle $\left(\theta_{\mathrm{i}}=\theta_{\mathrm{s}}\right)$ for three different scattering models.

FIGURE 4.6-6. Scattering patterns of a PEC plate as a function of observation distance and scattering angle for incidence angles of $0,30,60$, and 80 degrees computed with physical optics. Increasing intensity denotes increasing scattered power. Note that for $\Delta>1$, the maximum scattered power is in the specular direction $\left(\theta_{\mathrm{s}}=\theta_{\mathbf{i}}\right)$.

FIGURE 4.6-7. Scattering patterns of a PEC plate as a function of observation distance and scattering angle for incidence angles of $0,30,60$, and 80 degrees computed by the uniform theory of diffraction. Increasing intensity denotes increasing scattered power. Note that for $\Delta>1$, the maximum scattered power occurs in the specular direction $\left(\theta_{\mathrm{i}}=\theta_{\mathrm{S}}\right) .123$

FIGURE 4.6-8. Scattering patterns of a PEC plate as a function of observation distance and scattering angle for incidence angles of $0,30,60$, and 80 degrees. computed by the non-coherent scattering model. Increasing intensity denotes increasing scattered power. Note that the maximum scattered power always occurs in the specular direction $\left(\theta_{\mathrm{i}}=\theta_{\mathrm{s}}\right)$.......124

FIGURE 4.6-9. Reflection coefficient correction factor for slightly rough surfaces as a function of surface roughness in wavelengths and incidence angle. For slightly rough surfaces $\left(\sigma_{\mathrm{h}} / \lambda<0.2\right)$, this factor can be used to calculate the reduction of coherent power in the specular direction. 126

FIGURE 5.1-1. Example of the AutoCAD file as obtained from Facilities Planning and Construction.

FIGURE 5.1-2. Two-dimensional representation of major building features drawn on the BUILDING later in AutoCAD.

FIGURE 5.1-3. Three-dimensional AutoCAD representation of the second floor of Whittemore Hall on the Virginia Tech campus.

FIGURE 5.1-4. Sample ray tracing input file (.ray) ..............................................135

FIGURE 5.1-5. A building wall represented as a parallelogram. The location vector $l o c$ and parallel edge vectors $v 1$ and $v 2$ define the wall in the building database.

FIGURE 5.2-1. Spherical coordinate system used to reference the ray departure and arrival angles at the transmitter and receiver.

FIGURE 5.2-2. Ideal wavefront represented by each source ray (diagram from [Sch92]. 
FIGURE 5.2-3. Two views of a regular icosahedron that is inscribed inside a unit sphere. Rays are launched at icosahedron vertices.

FIGURE 5.2-4. Tessellation of icosahedron face. Ray wavefronts are hexagonal for edge and interior vertices.

FIGURE 5.2-5. Nearly hexagonal ray wavefront projected onto the surface of the unit sphere.

FIGURE 5.2-6. Maximum and minimum ray separation between nearest neighbors as a function of the number of rays traced. The difference between the maximum and minimum is caused by the difference between the pentagonal and hexagonal shaped wavefronts.

FIGURE 5.2-7. Difference between maximum and minimum ray separation between nearest neighbors as a function of the number of rays traced.

FIGURE 5.2-8. Ray tree that shows how one source ray can be decomposed into many transmitted, reflected, and scattered rays from intersections with planar boundaries.

FIGURE 5.2-9. Transmitted, reflected, and diffracted ray paths.

FIGURE 5.2-10. Transmitter and receiver in the presence of a specularly reflecting surface. A ray tracing solution must include only one specularly reflected ray for a given surface.

FIGURE 5.2-11. Two dimensional view of the reception sphere. The total ray path length is $\mathrm{d}$ producing a reception sphere radius of $\mathrm{ad} / \sqrt{3}$ (from [Sch92]).

FIGURE 5.2-12. Illustration of assumed wavefront for the purposes of the reception sphere. There is slight overlap between adjacent rays.

FIGURE 5.4-1. Sample ray tracing output file (.RAW).

FIGURE 5.4-2. Sample ray tracing sorted impulse file (.sort).................................154

FIGURE 5.4-3. Sample ray tracing power delay profile file (.pdp file). .155

FIGURE 6.1-1. Measured and predicted power delay profiles and the error curve as a function of excess delay measured on the second floor of Whittemore Hall on the Virginia Tech campus.

FIGURE 6.2-1. Magnitude of the Fresnel transmission and reflection coefficients at $4.0 \mathrm{GHz}$ for the effective building material properties of $\varepsilon_{\mathrm{r}}=4.4$ in Whittemore and $\varepsilon_{\mathrm{r}}=7.4$ in Norris.

FIGURE 6.2-2. Average and square root of the mean square error function of the difference between measured and predicted power delay profiles as a function of excess delay.

FIGURE 6.3-1. Measurement locations on the second floor of Whittemore Hall at 1.3 $\mathrm{GHz}$. The transmitter is indicated by a ' $\mathrm{Tx}$ ', and the receiver locations are indicated by the letters ' $A$ ' through ' $I$ '. 
FIGURE 6.3-2. Measured and predicted power delay profiles at location $G$ in

Whittemore Hall at $1.3 \mathrm{GHz}$. The path loss error is $1.8 \mathrm{~dB}$ and the rms delay spreads are nearly identical.

FIGURE 6.3-3. Measured and predicted power delay profiles for obstructed location ' $\mathrm{H}$ ' at $1.3 \mathrm{GHz}$ in Whittemore Hall. The path loss error is $0.3 \mathrm{~dB}$, and the arrival times of significant individual multipath components are predicted at total delays of up to $200 \mathrm{~ns}$.

FIGURE 6.3-4. Building blueprint of the second floor of Whittemore Hall that shows where power delay profile measurements were made at $4.0 \mathrm{GHz}$ with a spread spectrum channel sounder with a $8 \mathrm{~ns}$ pulse resolution.

FIGURE 6.3-5. Measured and predicted power delay profiles at receiver measurement location W251 in Whittemore Hall at $4.0 \mathrm{GHz}$. The path loss error is less than $1.0 \mathrm{~dB}$ and the rms delay spreads are quite similar.

FIGURE 6.3-6. Comparison of measured and predicted power delay profiles in Whittemore Hall at $4.0 \mathrm{GHz}$ at receiver location WJO.

FIGURE 6.3-7. Measured and predicted power delay profiles for the obstructed location WDA in Whittemore Hall at $4.0 \mathrm{GHz}$.

FIGURE 6.3-8. Building blueprint of Norris Hall that shows the transmitter and receiver locations where power delay profiles were recorded at 4.0 $\mathrm{GHz}$. At the end of the hallway near measurement location ' $\mathrm{H}$ ' was a large metal door. Measurements were made with the transmitter at locations ' $\mathrm{J}$ ' and ' $\mathrm{B}$ '

FIGURE 6.3-9. Measured and predicted power delay profiles for transmitter location ' $\mathrm{J}$ ' and receiver location ' $\mathrm{F}$ ' in Norris Hall at $4.0 \mathrm{GHz}$.

FIGURE 6.3-10. Measured and predicted power delay profiles for transmitter location

' $J$ ' and receiver location ' $H$ ' in Norris Hall at $4.0 \mathrm{GHz}$.

FIGURE 6.3-11. Measured and predicted power delay profiles in Norris Hall at 4.0 $\mathrm{GHz}$. The transmitter was at location ' $\mathrm{B}$ ', and the receiver was at location ' $\mathrm{H}$ '.

FIGURE 6.4-1. Scatter plot of measured and predicted path loss for the three sets of measurements in Whittemore Hall at 1.3 and $4.0 \mathrm{GHz}$ and in Norris Hall at $4.0 \mathrm{GHz}$. The standard deviation of path loss prediction error is $4.8 \mathrm{~dB}$ over all measurement locations.

FIGURE 6.4-2. Scatter plot of measured and predicted rms delay spread for all three measurement combinations.

FIGURE 6.4-3. Measured and predicted power delay profiles for location WHL1EVV in Whittemore Hall. Notice that the predicted component at $110 \mathrm{~ns}$ total delay is below the threshold leading to a large discrepancy between the measured and predicted rms delay spread. 178

FIGURE 6.4-4. Scatter plot of the measured and predicted COST 207 delay interval for $90 \%$ of the received energy. 
FIGURE 6.4-5. Scatter plot of the measured and predicted COST 207 delay interval for $75 \%$ of the received energy.

FIGURE 6.4-6. Scatter plot of the measured and predicted COST 207 delay interval for $50 \%$ of the received energy. 


\section{List of Tables}

TABLE 2.2-1: Table of the variables and the descriptions used to explain a discrete multipath channel model

TABLE 2.3-1: Summary of the variables used to describe the mean path loss exponent.

TABLE 2.4-1: Summary of variables used in the calculation of mean excess delay and $\mathrm{rms}$ delay spread.

TABLE 2.5-1: Table of variables and descriptions used to describe the computation of path loss from a wide band power delay profile.

TABLE 3.1-1: Summary of path loss and delay spread measured in a variety of buildings.

TABLE 3.5-1: Values of mean path loss exponent $n$ and standard deviation $\sigma$ for SIRCIM [Rap90b].

TABLE 3.6-1: Multi-floor path loss exponents measured in [Sei92a]. Path loss values from both buildings were combined to provide average multi-floor path loss exponents.

TABLE 3.6-2: Table of same-floor mean path loss exponents from [Sei92a].............50

TABLE 3.6-3: Table of Floor Attenuation Factor measured in five buildings in [Sei92a] and [Sei92c]. Note that the method used to determine the FAF was slightly different in [Sei92c] than in [Sei92a].

TABLE 3.6-4: Summary of the variables used to describe the soft partition and concrete wall attenuation factor model.

TABLE 3.6-5: Shadowing effects of common factory equipment measured at 1300 $\mathrm{MHz}$ in [Rap89a].

TABLE 3.6-6: Shadowing effects of typical factory obstructions measured at 1300 $\mathrm{MHz}$ in [Haw91].

TABLE 3.6-7: Shadowing effects of typical office building obstructions measured at $1300 \mathrm{MHz}$ in [Haw91].

TABLE 4.2-1: Table of variables and descriptions for geometrical optics.

TABLE 4.2-2: Table of variables and definitions for the description of free space propagation.

TABLE 4.2-3: $\quad$ Summary of variables used to describe plane wave reflection in equations (4.2-16) through (4.2-23).

TABLE 4.2-4: Table of loss tangent and relative permittivity for dry cement over a frequency range of $1 \mathrm{kHz}$ to $10 \mathrm{GHz}$.

TABLE 4.2-5: Table of variables and descriptions used to describe the two-ray planeEarth reflection model.......................................................................83

TABLE 4.5-1: Table of variables used in the bistatic radar equation........................104 
TABLE 5.2-1: Summary of the variables used to describe the ray tracing propagation model.

TABLE 6.3-1: Measured and predicted path loss, rms delay spread, and $90 \%$ delay interval at $1.3 \mathrm{GHz}$ on the second floor of Whittemore Hall. 168

TABLE 6.3-2: $\quad$ Measured and Predicted path loss, rms delay spread, and $90 \%$ delay interval at $4.0 \mathrm{GHz}$ on the second floor of Whittemore Hall. 171

TABLE 6.3-3: Measured and Predicted path loss, rms delay spread, and $90 \%$ delay interval at $4.0 \mathrm{GHz}$ on the first floor of Norris Hall. 


\section{Introduction}

This dissertation describes a geometrical optics model to predict propagation in buildings for personal communication system (PCS) design. It begins with a discussion of the growth of wireless communications and the importance of accurate propagation models in Chapter 1. The peculiarities of propagation in mobile and portable radio environments, particularly multipath propagation and its effects on transmitted signals, are discussed in Chapter 2. Multipath propagation measurements are also discussed in Chapter 2. Chapter 3 describes the state of the art of statistical and site-specific propagation models that can be used to design wireless communication systems. Chapter 4 presents some theoretical models for radiowave propagation in the presence of scattering bodies. The application of these models to a mobile and portable radio propagation environment is discussed, and scattering patterns are computed for smooth rectangular flat plates that represent building (wall) faces.

The geometrical optics ray tracing model which is the focus of this dissertation is described in Chapter 5. A graphical ray tracing program has been modified to predict multipath impulse responses based on building blueprints. The measured and predicted propagation characteristics are compared in Chapter 6. Chapter 6 presents a method for computing the effective building material properties for the walls in two different buildings. These effective building material properties lead to the reflection coefficient models that give the 'best fit' between measured and predicted propagation as determined from an error function that includes both multipath component amplitudes and arrival times. The ray tracing prediction model is shown to predict path loss with a standard deviation of less than $5 \mathrm{~dB}$. Time delay comparison shows that the amplitudes and time delays of measured power delay profiles can be predicted via ray tracing. The dissertation concludes with suggestions for improving the accuracy and expanding the applicability of the models presented here to wireless in-building propagation prediction. 


\subsection{The Growth of Wireless Communications in Recent Years}

In the $20^{\text {th }}$ century, mobile communications has experienced tremendous growth. The advent of wireless dispatch helped police and fire departments, and taxicab companies provide more efficient services to their customers. The use of paging transmissions provides a simple one-way message service to active persons such as medical personnel whose presence may be required on short notice. The use of pagers has increased 30 to $70 \%$ per year for the past three years [Rap91b].

In the 1970's, citizens band (CB) radio became a popular form of mobile communications. However, the allocated radio spectrum quickly became crowded, and radio coverage was limited since a user can only talk to persons within range of his or her own transmitter. There is no system infrastructure to transfer calls over long distances or to specific persons. The only way to receive a message is for the user to continuously monitor a specific radio channel.

Established in the U.S. in 1983, cellular telephone systems have provided mobile access to the fixed telephone network for users in automobiles. Hand-held portable devices have become extremely popular. In the past three years, cellular telephone companies have experienced growth rates of 33 to 50\% [Rap91b]. From late 1989 to late 1991, the number of users in the U.S. grew from 2.5 million to 6.3 million. This growth has been rapid as there were only twenty-five thousand users in 1984 . In Sweden, $6.6 \%$ of the population own cellular telephones [Rap91b].

Recently, cordless telephones have become a pervasive technology in many homes. Over sixty-five million cordless telephones have been sold, although it is believed that over half have been discarded and are no longer used. These handsets allow a limited range of portability as they must remain within range of a particular base station that is connected to the fixed telephone network.

The future of wireless personal communications consists of merging the best features of cordless telephones and cellular radio. A small low power handset with a long talk time that operates in a variety of environments with continuous access to a fixed network 
is envisioned [Rap91b]. This concept is termed a personal communications network (PCN) or personal communications system (PCS). Each user would have his or her own private telephone number. In this way, it would be possible to telephone a person instead of a place where that person is expected to be. Recently, large corporations have expressed intense interest in research and development of personal communications systems that will be available in a few years. The development of such systems requires engineering tools and techniques that allow rapid accurate propagation prediction and system design [Rap91b]. The Federal Communications Commission (FCC) has recently issued many experimental licences for corporations to perform propagation and system experiments in the PCS area [Te191]. The FCC has even proposed reallocating under-utilized existing fixed line-of-sight microwave link spectrum to be shared with PCS [Mic92]. For successful application of this concept, mutual interference levels must be kept to a minimum. Hence, accurate propagation models are required to determine interference levels for a given base station placement so that an optimum design may be achieved. With the extreme growth of existing wireless communications systems, it is believed that billions of dollars could be made from the deployment of wireless personal communications systems. In order to make these systems a reality, the propagation of radio signals must be well understood. The transition from a wireline communications system to a wireless one consists of replacing a wire that has a slowly varying known impulse response with an unknown highly varying impulse response.

Wireless communications systems have an advantage over wired communications systems. This advantage is mobility. With mobility, a person can make or receive phone calls when and where he or she desires. In an increasingly information-based service-oriented society, rapid communications is at a premium. The ability to make a call anywhere and anytime can increase productivity and improve public safety as emergency help can be summoned quickly. Although cellular radio was at one time viewed as a toy for the rich, rapid growth has meant more common acceptance of cellular technology. With a widely deployed personal communications system, infrastructure costs can be spread out over many more users thus making PCS affordable for the masses. Accurate propagation prediction techniques are required to reduce installation costs and time dramatically. 


\subsection{The Cellular Concept}

In order to realize the full potential of PCS, it is essential that accurate propagation models are developed which consider the specific mobile environment. In conventional mobile radio systems, a single high power base transmitter mounted on a tall tower or on top of a tall building serves a large coverage area. Coverage areas are generally several tens of kilometers in diameter. This keeps system costs down since few base stations are required. However, the number of users that can be served is small since only a limited number of radio channels (frequencies) are available. In cellular radio systems, a coverage region is divided into smaller areas. Figure 1.2-1 shows a coverage region divided into smaller contiguous hexagonal shaped regions called cells. Each cell has its own base transmitter. The advantage that cellular radio systems have over conventional mobile radio

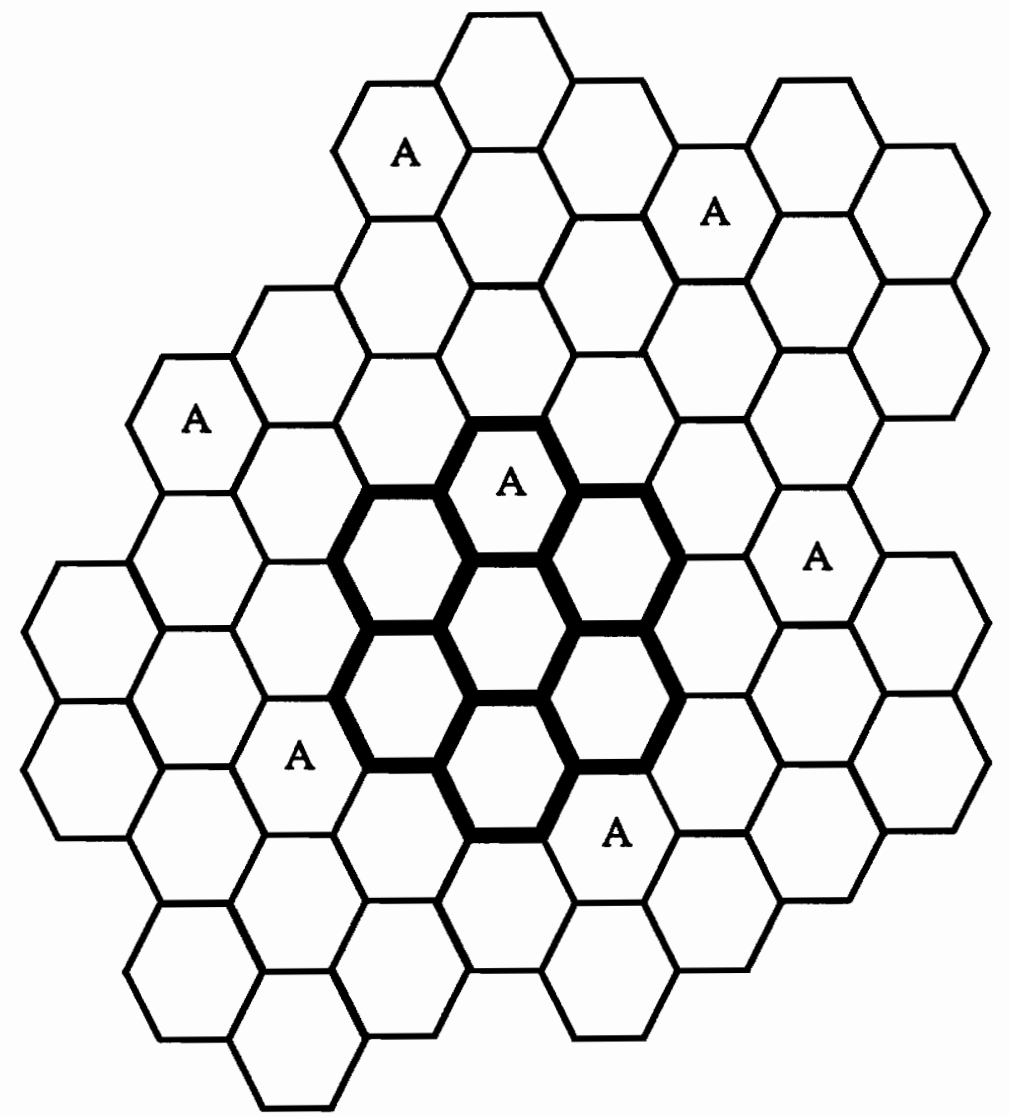

FIGURE 1.2-1. A seven frequency re-use pattern used in cellular radio. The same frequencies are used in all cells marked with the letter ' $A$ '. 
systems is that the same radio channels can be used for more than one base station in a coverage area. For example, in Figure 1.2-1, the cells marked with the letter ' $A$ ' all use the same set of radio channels. This pattern is known as a seven cell frequency re-use pattern where the same channels are used every seven cells [Lee89]. Although a cellular system is much more complex than a conventional mobile radio system, the result is that many more mobile subscribers can be served. For example, suppose the region in Figure 1.2-1 represents a city. With the cellular layout shown, up to seven different calls may be active at any one time on a given radio channel. A conventional mobile radio system can only support one call per radio channel. Thus, a cellular radio system that re-uses frequencies, uses a finite natural resource (spectrum) more efficiently than a conventional mobile radio system. A microcellular system employs smaller cells and lower antenna heights to increase the number of cells in a coverage area.

In order for a cellular system to operate efficiently, the radio propagation between cells must be limited since the radio signals from one cell are interference signals in another. Future personal communications systems will likely use low power and low antenna heights to limit the propagation outside the desired coverage area. In future personal communications systems which utilize the cellular concept, it will be essential that the propagation characteristics be known in advance so that systems may be designed to provide service comparable to that of the wireline telephone system.

\subsection{The Importance of Accurate Propagation Models}

Hard wired communications systems use transmission lines to connect communications terminals. The effects of transmission lines on the transmitted signal are well known [Lia85]. As tetherless personal communications systems evolve, the transmission line with a known impulse response is replaced with a radio interface with an impulse response that is constantly changing as the user roams throughout the coverage area of the system. This impulse response is often unknown.

A received signal in a multipath environment can change significantly with relatively small changes in the surroundings. The locations of objects that surround transmit- 
ters and receivers severely affect the propagation characteristics of any radio channel. The propagation characteristics of mobile and portable radio channels are discussed in more detail in Chapter 2. The performance of in-building high-capacity wireless communications is limited by the propagation characteristics. Thus, it is important to understand how the physical surroundings affect the propagation environment. Once the propagation is understood, systems may be designed more efficiently in terms of site layout, frequency planning, and system performance. The economic success of a commercial mobile radio service is closely related to the price/performance ratio of the service. If system costs such as site design and installation are high, these large fixed costs will decrease the net profit. If service quality is poor, subscribers will leave the system, and there will be little or no income.

Accurate propagation models can allow researchers to determine the appropriate modulation, equalization, and multiple access techniques that are most suited for wireless systems in multipath propagation environments. System throughput and acceptable bit error rate will likely be application specific, and the appropriate propagation models will be needed to efficiently design these systems [Ste91].

In current cellular systems, accurate site-specific propagation models do not exist. Either exhaustive measurements throughout a coverage region are performed, or crude statistical models are used to determine propagation characteristics. Systems must be over-designed and hence are less efficient in terms of frequency re-use. This leads to increased system installation costs and decreased system capacity. Future installation techniques will rely on site-specific theoretical and experimental computer propagation models that incorporate the particular environment in the propagation prediction.

In future wireless systems, it is likely that all cells will use the same frequency and wide band code division multiple access (CDMA) technique. In CDMA, all users share the same bandwidth, but each user has a specific pseudo-noise code that is used to access the communications channel. Signals other than the desired signal contribute to the background noise. In current cellular systems, interference comes from users who are using the same channel in a different area. This is called co-channel interference. Systems are 
designed to reduce the interference from a few users in known (to within a cell radius) locations. With CDMA, all users are interferers, but the interference caused by each user is smaller than in a narrow band system. More users can be accommodated for a given amount of spectrum in a certain coverage area [Rap90c]. In CDMA systems, interferers are located in all cells, not just a few specific ones. Thus, the propagation from each cell to every other must be known so that system designers can reduce interference between neighboring cells.

For a personal communications system in a building, knowledge of propagation characteristics a priori could allow evaluation of proposed building changes or construction based on the radio properties of the building. Since it is not likely that major building design changes will be determined according to radio specifications, at least the required changes in the radio system could be known before construction began.

This chapter has described the rapid changes in wireless communications systems over the past few years. Currently, system design and installation is an expensive timeconsuming process. As wireless personal communication systems evolve, rapid inexpensive deployment is necessary for system operators to make a profit providing high quality affordable service. For this to happen, accurate propagation prediction tools must be developed. This dissertation presents a geometrical optics propagation model to accurately predict wireless impulse responses in buildings. Results will show that the power delay profile can be predicted with a standard deviation of path loss error of less than $5 \mathrm{~dB}$ based on site-specific knowledge of wall locations and building materials. Successful application of such a tool would make PCS both affordable for the user and profitable for the system operator. 


\section{The Mobile and Portable Radio Propagation Environment}

\subsection{Overview}

In a mobile and portable radio environment, one communications terminal is allowed to roam throughout a coverage area with tetherless access to the communications system through a network of fixed base stations. Mobile and portable radio channels suffer from multipath propagation. This chapter discusses multipath propagation, its effects on transmitted signals, and methods used to measure and model the propagation.

This chapter begins with a discussion of multipath propagation and the effects of multipath propagation on signals as a function of bandwidth. These effects are then considered in the development of a discrete multipath channel model. The definition of radio path loss between a transmitter and receiver is given, and a common statistical model for path loss as a function of distance is presented. Multipath channel parameters that quantify the time dispersion of the transmitted signal are determined from a power delay profile representation of the channel impulse response. Methods for measuring multipath propagation and determining the multipath channel parameters from the received data are described.

\subsection{Multipath Propagation}

In buildings, radio waves are attenuated and redirected as they are scattered by walls, partitions, and other obstacles. Consider a radio transmitter and receiver located in a multipath scattering environment as shown in Figure 2.1-1. The signal leaves the transmitter antenna and arrives at the receiver antenna via multiple paths caused by scattering from objects that surround the transmitter and receiver. This is known as multipath propagation. Each individual multipath signal is attenuated and time delayed as it travels through and scatters off of the objects in the environment. Thus, the received signal is a series of attenuated, time-delayed replicas of the transmitted signal. Figure 2.1-1 illustrates two possible paths between the transmitter and receiver. One is the direct path which is the shortest distance between the transmitter and receiver. The other path is scattered by a surrounding 


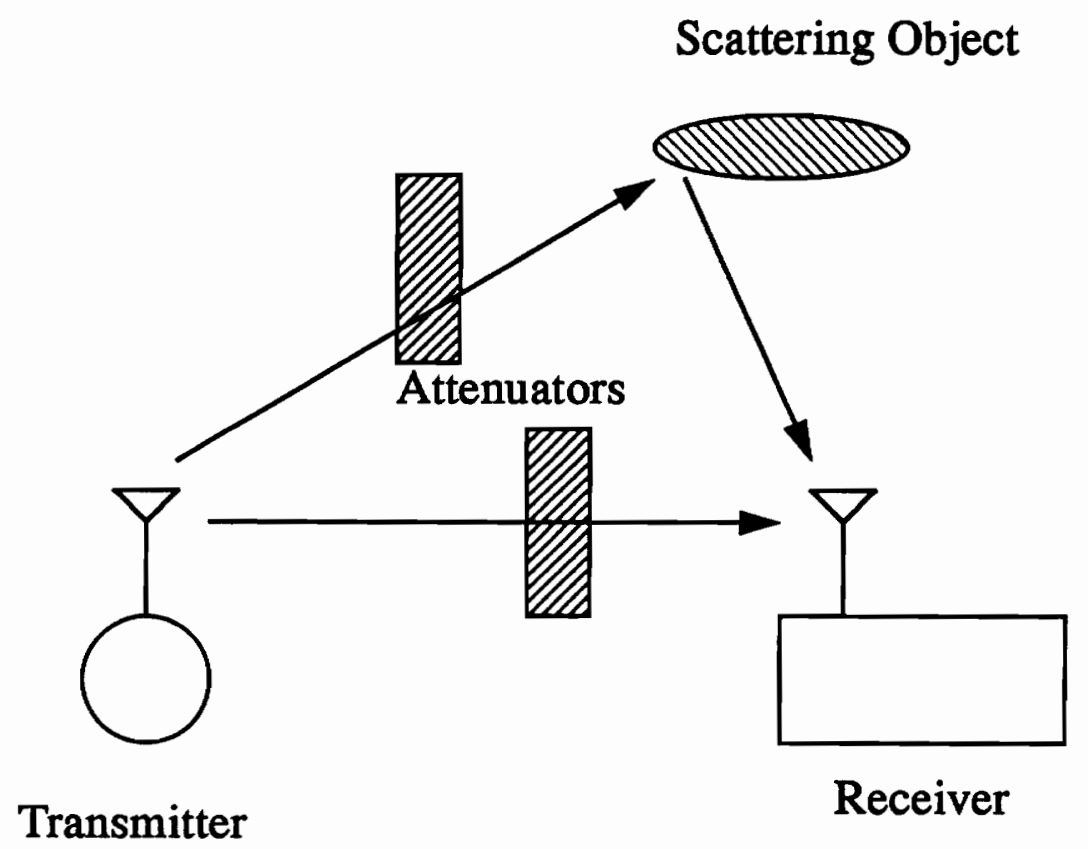

FIGURE 2.1-1. A transmitter and a receiver located in a multipath scattering environment (from [Dev87c]).

object. Both paths are attenuated by passing through obstructing objects that are not transparent to radio signals. In buildings, such objects may be static such as walls, partitions, furniture, and factory equipment, or time-varying like people moving around. Multipath propagation is not limited to radio terminals located inside buildings, but is a characteristic of all mobile and portable radio propagation environments. Cellular radio signals undergo multipath propagation caused by reflections and scattering from buildings and mountains. Diffraction around street corners can also create multiple paths between transmitters and receivers. Line-of-sight (LOS) point-to-point microwave radio links also suffer from multipath propagation due to a ground reflected path and multiple ray paths through the atmosphere due to a change in the refractive index with altitude in addition to the direct path. Ionospheric radio channels suffer from multipath propagation caused by scattering from millions of ionized particles in the ionosphere.

When a narrow band (CW) signal is transmitted in a multipath environment, the amplitude of the received signal undergoes many rapid variations as either the transmitter 
or receiver is moved, or as the transmitter and receiver remain stationary and objects in the channel move. Figure 2.1-2a shows the amplitude variation with motion. The received sig-

a.)

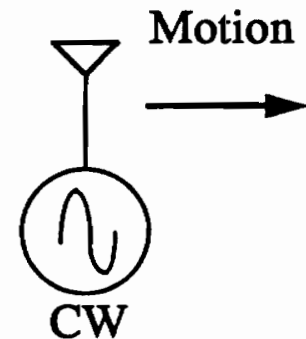

Transmitter

b.)<smiles>CC12C=CC(CC1)C(C1CC1)C(C1CC1)CC2</smiles>

Swept CW

Transmitter

c.)<smiles>c1ccc(C2CC2)cc1</smiles>

Pulse

Transmitter

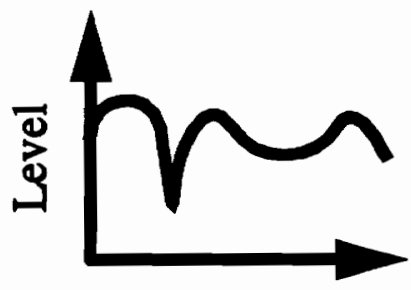

Motion
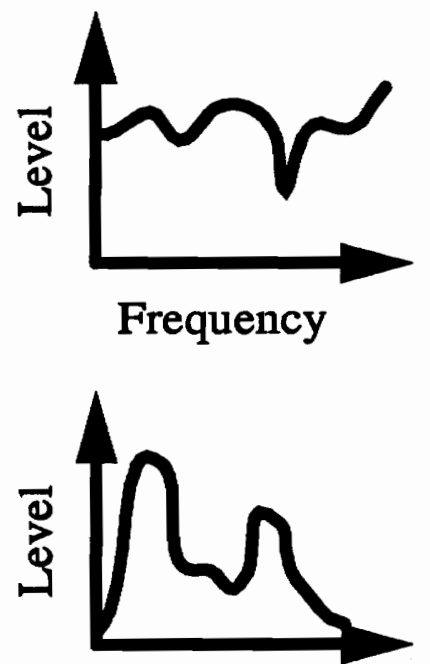

Time

FIGURE 2.1-2. The effects of multipath propagation on the received signal for different types of transmitted signals (from [Dev87c]).

nal is the phasor sum of the individual multipath components incident upon the receiver antenna. The rapid amplitude variation is due to the phase variations of each received multipath signal as one terminal is moved or objects in the channel move. The phase $(\phi)$ of each individual multipath component is

$$
\phi=\phi_{0}+\frac{2 \pi l}{\lambda}
$$


where $\phi_{0}$ is a random phase uniformly distributed over $[0,2 \pi), l$ is the path length, and $\lambda$ is the carrier wavelength. As the path length of each multipath component changes with motion, the phases change proportionately and the phasor sum changes rapidly.

If both terminals are stationary, and the transmitted CW signal is swept across a frequency range, amplitude variations are observed as the frequency is changed. Figure 2.1-2b shows amplitude variations as the carrier frequency is swept over a finite range. In this case, the rapid phase variations are caused by the change in carrier wavelength in equation (2.1-1) as the frequency is swept.

The maximum width in the frequency band in Figure 2.1-2b in which the statistical properties of the received signal are highly correlated is called the coherence bandwidth $\left(B_{C}\right)$ [Gan72]. Two sinusoids will be affected similarly by a multipath channel if their frequency difference is less than the coherence bandwidth [Rap87]. If the bandwidth of a transmitted signal is less than the coherence bandwidth, the signal is impaired only by multipath amplitude fading. If the bandwidth is greater than the coherence bandwidth, adjacent symbols (in digital transmissions) overlap and cause additional bit errors from intersymbol interference (ISI). If two signals are separated by the coherence bandwidth or greater, one signal may be strong and another may be weak. This is termed frequencyselective fading.

Consider the transmission of a narrow pulse. If the pulse width is less than the difference in arrival times between individual multipath components, then the individual components can be distinguished by their time delays. A wide bandwidth is required to transmit a narrow pulse. Multiple attenuated time-delayed versions of the transmitted signal can be seen in the received signal. Figure 2.1-2c shows the amplitude variations with time when a narrow pulse is transmitted.

The probing signals in Figure 2.1-2b and $\mathrm{c}$ are Fourier transform pairs. The inverse Fourier transform of the swept CW signal is a pulse, and the Fourier transform of the pulse is a swept CW spectrum. Hence, the received signals are also duals of one another. The Fourier Transform of a multipath impulse response leads to nulls in the frequency 
response. Figure 2.1-3 shows a multipath impulse response and the corresponding frequency spectrum. The time impulse response is denoted as $P(\tau)$, and the frequency response is denoted as $S(f)$.
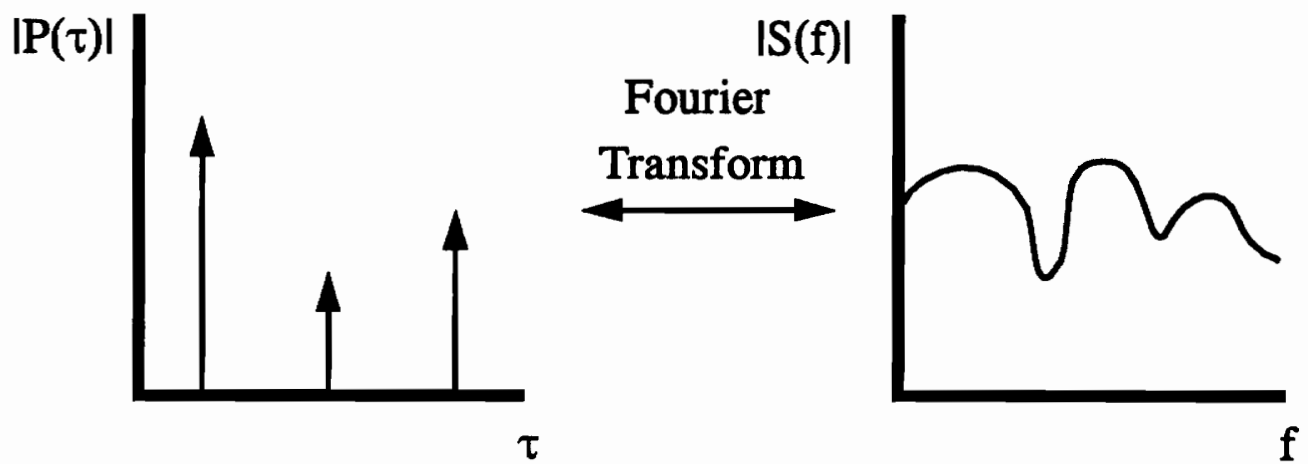

FIGURE 2.1-3. Multipath channel impulse response and frequency spectrum.

It is important to understand the effects of bandwidth on propagation in mobile and portable radio environments. The RF bandwidth of a probing signal determines the baseband time resolution, $\mathrm{T}_{\mathrm{bb}}$, of propagation measurements. $T_{b b} \approx \frac{2}{B_{R F}}$ with the approximation due to real-world filters. For a narrow band signal ( $\mathrm{B}_{\mathrm{RF}}$ on the order of $15 \mathrm{kHz}$ ), there is essentially no time resolution since the pulse resolution is much larger than the excess delays found in a mobile radio environment. The instantaneous amplitude and phase can be continuously measured. The receiver performs a phasor sum of all $\vec{E}$ fields incident upon the receiver antenna. The resultant signal amplitude fades rapidly due to the constructive and destructive interference of the $\vec{E}$ fields and is highly dependent upon the exact small-scale location of the transmitter, receiver, and surrounding objects (recall Figure 2.1-2a). This interference is caused by the rapid phase change of each received multipath component. For a wide band signal $\left(B_{R F}>B_{C}\right)$, the receiver is able to distinguish the time delays of individual multipath components to a resolution of $T_{b b}$. Figure 2.1-4 shows a pulsed RF signal in the time domain. A sine wave at the RF carrier frequency is turned on for $\mathrm{T}_{\mathrm{bb}}$ seconds and is then turned off. The complex envelope tracks the low frequency variations of the signal envelope. 


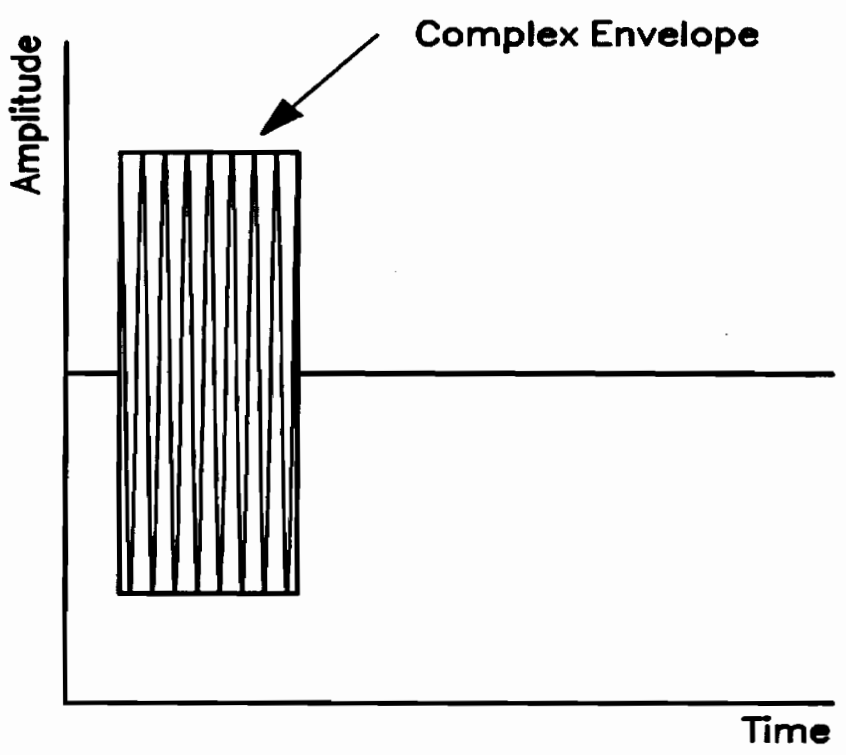

FIGURE 2.1-4. Complex envelope of a pulsed RF signal. The complex envelope is the low-pass representation of the amplitude variations on an RF carrier.

Consider Figure 2.1-5a to represent an impulse response measurement with an infinite bandwidth (perfect impulse). Suppose Figure 2.1-5a represents the true impulse response of a propagation channel. If propagation measurements are made with $250 \mathrm{MHz}$ $\mathrm{RF}$ bandwidth, the impulse response has an $8 \mathrm{~ns}$ resolution as shown in Figure 2.1-5b and the individual multipath components are resolvable. In Figure 2.1-5c, the first two pulses are resolvable but start to run together, and the third pulse is nearly lost with the 30 ns pulse width. Figure 2.1-5d shows that with a pulse width of $85 \mathrm{~ns}$, the three multipath components are indistinguishable. Note that the received pulses are rounded due to the finite bandwidth of receiver filters.

The propagation time delay is proportional to the path length traveled by the multipath component. The path length $l=c \tau$, where $\tau$ is the time delay, and $c=3 \times 10^{8}$ is the speed of light in a vacuum (also free space). A rule of thumb used to distinguish path length from received power delay profiles is $1 \mathrm{ft}$. $\approx 1 \mathrm{~ns}$. Thus, a pulse width of $8 \mathrm{~ns}$ can resolve multipath components to within about $8 \mathrm{ft}$. and a pulse width of $85 \mathrm{~ns}$ can only resolve path length differences to within $85 \mathrm{ft}$. In an indoor environment, transmitters and receivers are generally within 100 meters of each other. Outdoors, transmitters and receivers may be separated by several kilometers. Since the transmitter and receiver are closer 

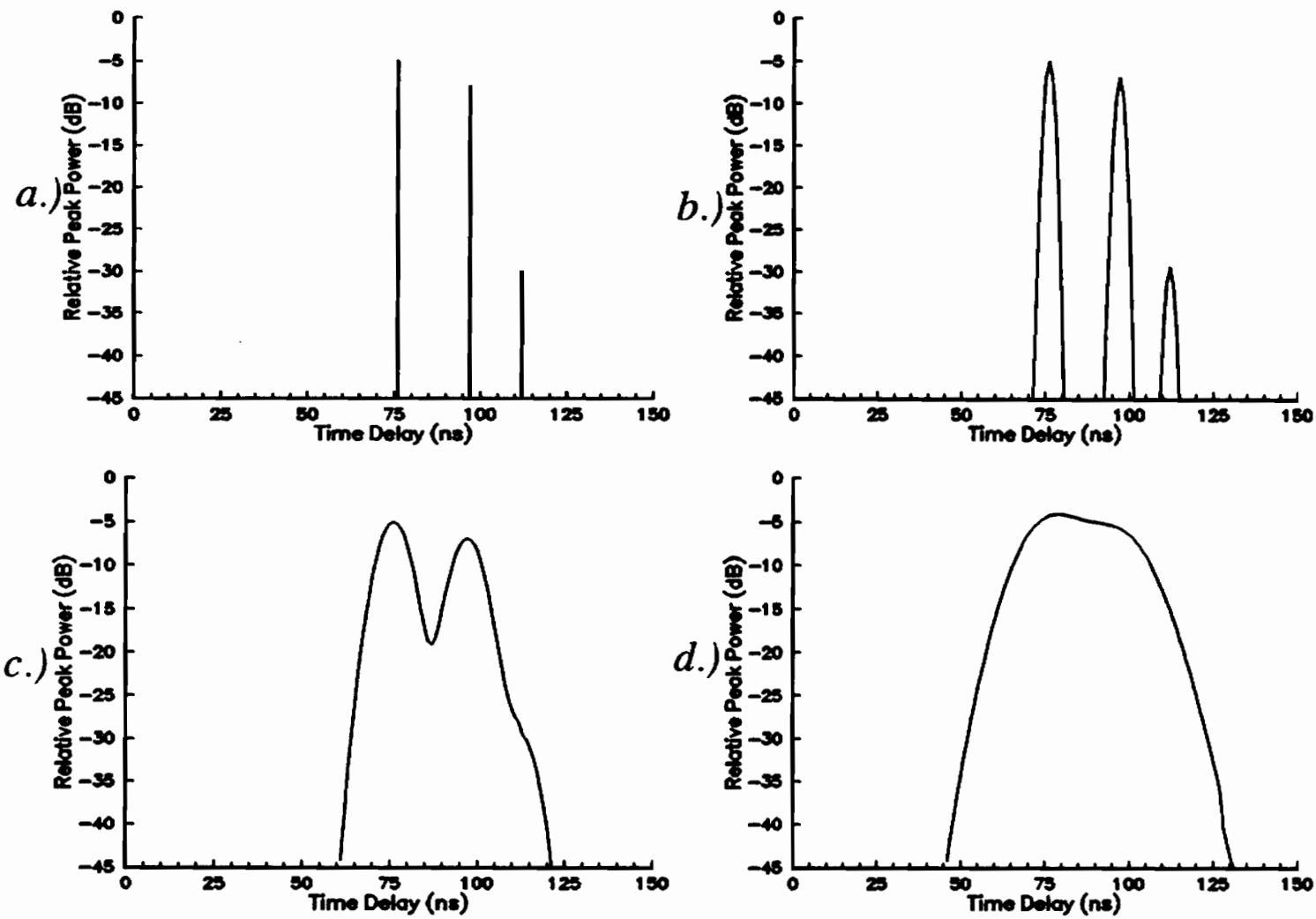

FIGURE 2.1-5. Impulse response estimate for various probing bandwidths. Part a.) shows a perfect impulse response, and parts b.), c.), and d.) show the effect of decreasing bandwidth on time resolution.

together in buildings, scattering objects that affect signal propagation are located closer to the transmitter and receiver. Hence, the time delays and time delay differences of multipath components are also generally smaller. In order to resolve individual multipath components a narrower pulse (wider bandwidth) should be used for characterizing indoor environments than outdoor ones. For example, a 500 ns pulse width may be acceptable for determining channel characteristics in large cell environments [Rap90a], [Sei91b]. However, in indoor environments pulse widths of $50 \mathrm{~ns}$ are common [Dev86], [Bul89], [Dev90b], and pulse widths as small as 5-10 ns have been used [Rap89b], [Haw91].

\subsection{Multipath Channel Model}

This section considers a discrete impulse response model for multipath channels. Consider a multipath channel to be a time-varying filter [Cou90]. Table 2.2-1 summarizes the variables used in the description of the multipath channel model. 
TABLE 2.2-1: Table of the variables and the descriptions used to explain a discrete multipath channel model.

\begin{tabular}{|c|c|}
\hline Variable & Descriplion Lunits) \\
\hline $\mathbf{x}(\mathrm{t})$ & Transmitted waveform [Volts] \\
\hline$y(t)$ & Received waveform [Volts] \\
\hline$h(t)$ & Impulse response of propagation channel \\
\hline$\alpha_{k}(t)$ & Multipath amplitude gain factor of the $k^{\text {th }}$ multipath component \\
\hline$\tau_{k}(t)$ & Excess delay of the $\mathrm{k}^{\text {th }}$ multipath component [seconds] \\
\hline$p(t)$ & Baseband probing pulse shape \\
\hline $\mathrm{T}_{\mathrm{bb}}$ & Pulse width of probing signal [seconds] \\
\hline $\mathbf{r}(\mathrm{t})$ & Baseband complex envelope of the received signal [Volts] \\
\hline $\mathbf{f}_{\mathrm{c}}$ & Carrier frequency $[\mathrm{Hz}]$ \\
\hline$\theta_{\mathbf{k}}$ & Phase of $\mathbf{k}^{\text {th }}$ multipath component [radians] \\
\hline$|r(t)|^{2}$ & Multipath impulse response estimate \\
\hline
\end{tabular}

Let $x(t)$ represent the transmitted waveform and $y(t)$ the received waveform, a mobile radio multipath channel may be characterized by the impulse response $h(t)$. The received signal $y(t)$ is an attenuated and time delayed version of the transmitted signal $x(t)$. For a discrete channel model, this implies that the received signal is a series of attenuated, time-delayed, phase shifted replicas of the transmitted signal [Rap89b], [Te191].

$$
y(t)=\sum_{k} \alpha_{k}(t) x\left(t-\tau_{k}(t)\right)
$$

Note that the received signal in $(2.2-1)$ is a function of both time and excess delay $\left(\tau_{k}\right)$. Individual impulse response snapshots are assumed to be time invariant, or at least wide-sense stationary, over a measurement interval (typically on the order of one second). Thus, the $\alpha_{\mathrm{k}}$ 's and $\tau_{\mathrm{k}}$ 's measured are random variables which are not functions of time, but are functions of the spatial location of the transmitter, receiver, and the scattering objects that surround them. Using this fact, each impulse response snapshot is given by 


$$
h(t)=\sum_{k} \alpha_{k} \delta\left(t-\tau_{k}\right)
$$

where $\delta()$ is the unit impulse function and $\tau_{0}$ is the arrival time of the first observable pulse.

Consider a transmitted signal of the form $x(t)=\operatorname{Re}\left[p(t) e^{j 2 \pi f_{c} t}\right]$ with baseband pulse width $\mathrm{T}_{\mathrm{bb}}$, where $p(t) \cong 1$ for $0 \leq t \leq T_{b b}$, and is zero elsewhere (recall Figure 2.14). The channel output is the convolution of the input signal and the impulse response

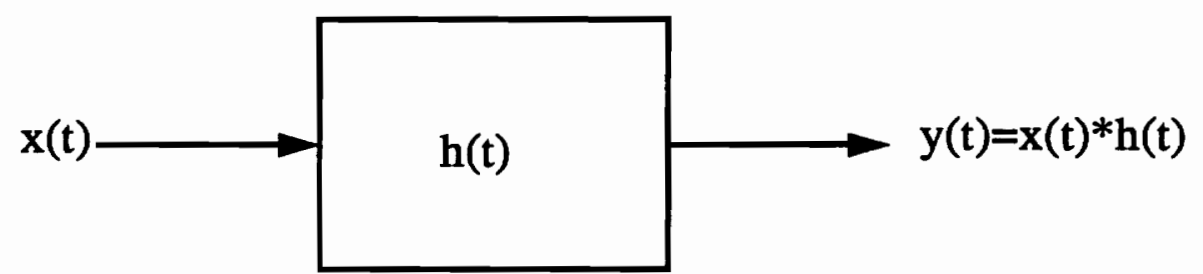

$$
y(t)=\int_{-\infty}^{\infty} x(\varsigma) h(t-\varsigma) d \varsigma=\operatorname{Re}\left[r(t) e^{j 2 \pi f_{c} t}\right]
$$

where the received complex envelope that represents the baseband channel impulse response estimate is

$$
r(t)=\sum_{k} \alpha_{k} e^{-j 2 \pi f_{c} \tau_{k}} p\left(t-\tau_{k}\right)
$$

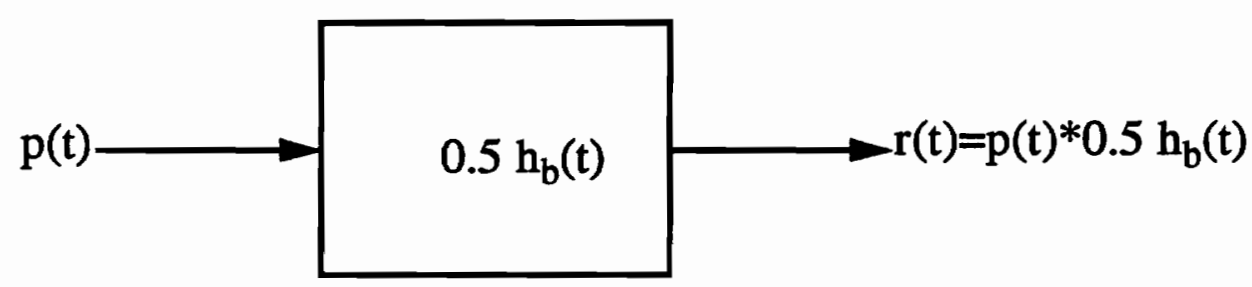

The channel may be equivalently described by the baseband impulse response $h_{b}(t)$ having an output $r(t)$ which is the complex envelope of $y(t)$. The factor 0.5 is due to the down conversion from RF to baseband. The low pass characterization removes the 
high frequency variations caused by the carrier, making the signal analytically easier to handle. Thus, the low pass equivalent channel impulse response $h_{b}(t)$ is given by

$$
h_{b}(t)=\sum_{k} \alpha_{k} e^{-j 2 \pi f_{c} \tau_{k}} \delta\left(t-\tau_{k}\right)
$$

In (2.2-5), $\alpha_{\mathrm{k}}$ represents a real attenuation factor, $e^{-j 2 \pi f_{c} \tau_{k}}$ represents a linear phase shift due to propagation time, and $\tau_{\mathbf{k}}$ is the time delay of the $\mathbf{k}^{\text {th }}$ multipath component. Additional phase shifts may be induced by reflection, transmission, and scattering of the radio signal.

When an RF carrier is modulated with a sounding probe of pulse width $T_{b b}$, the output of the low pass channel closely approximates the impulse response $h_{b}(t)$. Commonly, instead of measuring the output $r(t),\left.\operatorname{Ir}(t)\right|^{2}$ is measured [Sal87], [Rap89b], [Rap90a], [Sei91b]. This squared magnitude impulse response estimate is called a power delay profile.

Letting $\theta_{\mathrm{k}}$ represent the linear phase term in (2.2-5) plus an additional random phase, each multipath power delay profile snapshot becomes

$$
\begin{aligned}
|r(t)|^{2} & =r(t) r^{*}(t) \\
& =\operatorname{Re}\left[\sum_{j} \sum_{k} \alpha_{j} \alpha_{k} p\left(t-\tau_{j}\right) p\left(t-\tau_{k}\right) e^{-j\left(\theta_{j}-\theta_{k}\right)}\right]
\end{aligned}
$$

Note that if $\left|\tau_{\mathrm{j}}-\tau_{\mathbf{k}}\right|>\mathrm{T}_{\mathrm{bb}}$ for all $j \neq k$, then

$$
|r(t)|^{2}=\sum_{k} \alpha_{k}^{2} p^{2}\left(t-\tau_{k}\right)
$$

and the power delay profile measurement has a path resolution of $T_{b b}$. For $\left|\tau_{j}-\tau_{k}\right|<T_{b b}$, there is pulse overlap and it is assumed that there are unresolvable sub-paths that form one resolvable multipath component. Note that the sum in equation (2.2-7) is the power delay profile and appears similar to Figure 2.1-5. 


\subsection{Path Loss}

\subsubsection{Computing Path Loss}

Path loss is a quantitative measure of the amount of signal power that is lost as the signal propagates from the transmitter to the receiver. The path loss between a transmitter and receiver separated by some distance $d$ is the average of the instantaneous CW path loss, spatially averaged over a small-scale distance (several wavelengths). Path loss can also be computed from a time averaged wide band power delay profile. A narrow band multipath fading signal can be decomposed into a slowly-varying component and a rapidly varying component. The slowly varying component is obtained by a spatial average of the received signal. The amplitude of this slowly fading component is influenced primarily by the large-scale objects in the radio channel, and is commonly termed the shadowing component since the mean signal level is influenced when the receiver is shadowed from the transmitter.

The shadowed component is used to determine the path loss for a given measurement location. Figure 2.3-1 shows a $1900 \mathrm{MHz}$ signal that fades rapidly and the shadowed component for the particular measurement run. An averaging distance of $20 \lambda$ was used to compute the spatial average.

The portion of the signal that remains when the shadow fading is removed from the received signal is termed the fast fading signal. Fast fading is caused by the rapidly varying phasor sum due to the phase changes of the individual multipath components incident upon the receiver antenna. This term is also commonly called the Rayleigh component since the probability distribution of the signal amplitude is often Rayleigh distributed.

\subsubsection{Statistical Mean Path Loss Exponent}

In free space, path loss increases with distance as $20 \mathrm{~dB} / \mathrm{decade}$. When scattering objects are present, path loss can be very different from that in free space. For propagation measurements taken in a variety of locations where different propagation mechanisms 


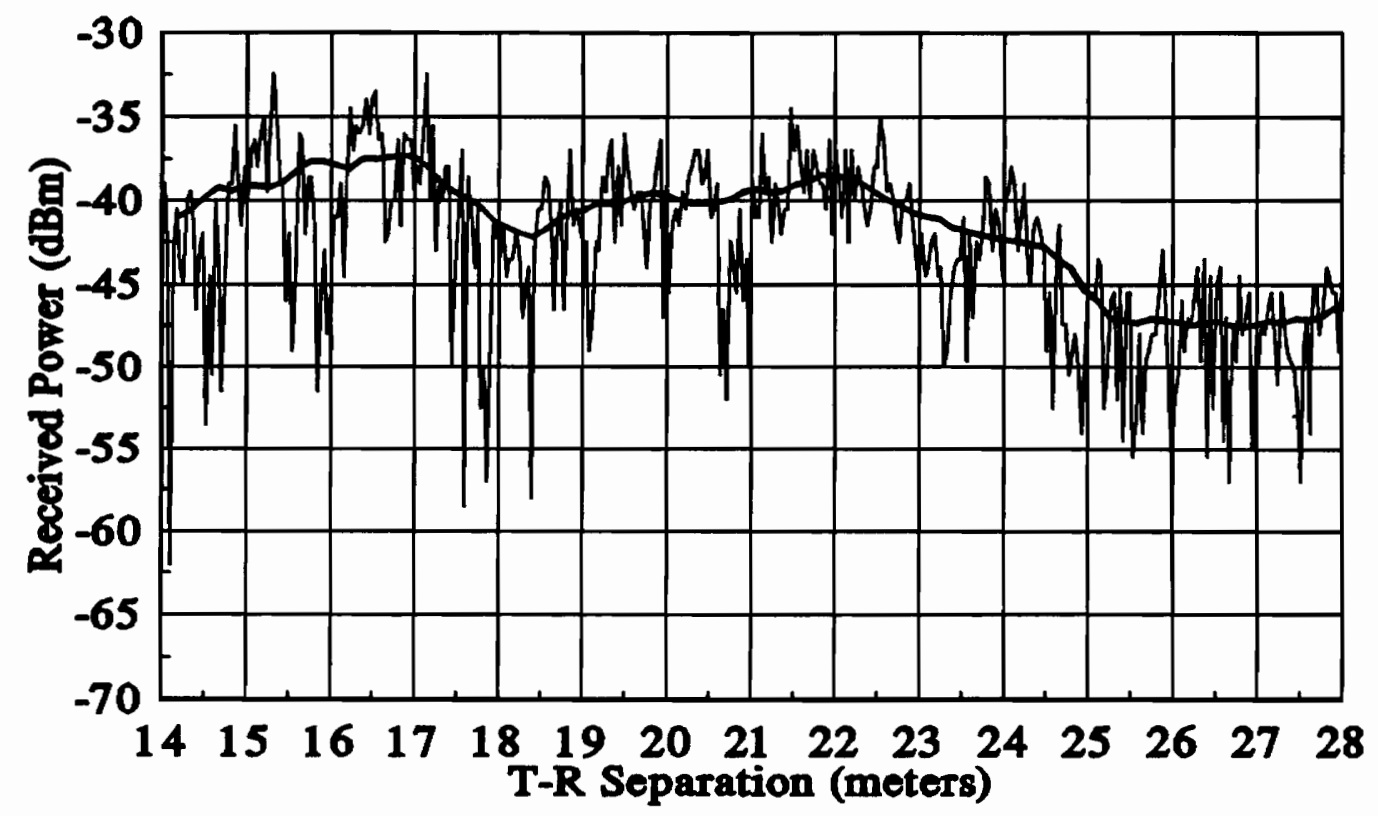

FIGURE 2.3-1. Example of $\mathrm{CW}$ fading. The local mean averaged over $20 \lambda$ is also shown.

influence path loss, it is necessary to develop a model that describes how mean path loss increases with distance.

A model used in [Ale83a], [Ale83b], [Cox85], [Bul87], [Mot88], [Arn89], [Chi89], [Owe89], [Pah89], [Rap89a], [Rap89b], [Haw90], [Mot90], [Rap91a], [Sei91b] indicates that mean path loss increases exponentially with distance. That is,

$$
\overline{\text { Path Loss }}(d) \propto\left(\frac{d}{d_{0}}\right)^{n}
$$

where $n$ is the mean path loss exponent which indicates how fast mean path loss increases with distance, $d_{0}$ is a reference distance, and $d$ is the transmitter-receiver (T-R) separation distance. When plotted on a log-log scale, this power law relationship is a straight line. Table 2.3-1 describes the variables used in the statistical mean path loss exponent model. 
TABLE 2.3-1: Summary of the variables used to describe the mean path loss exponent.

\begin{tabular}{|c|c|}
\hline Yariable & Bescription [unts] \\
\hline d & T-R separation [m] \\
\hline $\mathrm{d}_{\mathbf{0}}$ & Reference distance $[\mathrm{m}]$ \\
\hline PL & Path loss [dB] \\
\hline$\overline{P L}$ & Mean path loss $[\mathrm{dB}]$ \\
\hline$n$ & Mean path loss exponent \\
\hline$\lambda$ & Carrier wavelength [m] \\
\hline$\sigma$ & Standard deviation of path loss about the mean path loss [dB] \\
\hline
\end{tabular}

The mean path loss at a distance $d$ in decibels is an algebraic equation defined as the path loss in decibels from the transmitter to the reference distance $d_{0}$ plus the additional path loss described by (2.3-1) in decibels.

$$
\overline{\mathrm{PL}}(d)[\mathrm{dB}]=P L\left(d_{0}\right)+10 \times n \times \log _{10}\left(\frac{d}{d_{0}}\right)[\mathrm{dB}]
$$

The selection of the reference distance $d_{0}$ is critical in the interpretation of path loss measurements. Urban mobile radio propagation measurements in [Sei91b] show that a change in reference distance from $100 \mathrm{~m}$ to $1 \mathrm{~km}$ changes the perceived mean path loss exponent from 2.7 to 3.0. It is important to choose a reference distance that is appropriate for the propagation environment. In large cell cellular channels, $1 \mathrm{~km}$ and 1 mile reference distances are commonly used [Lee82], [Lee89], [Rap90c], [Sei91b]. For microcellular measurements in [Sei91b], $d_{0}=100 \mathrm{~m}$. For measured data in microcellular and indoor environments, a $1 \mathrm{~m}$ reference distance has been used [Haw90], [Haw91], [Sei91a], [Te191], [Sei92a]. A reference distance should be chosen so that it is in the far-field of the antenna $\left(d_{0}>2 \mathrm{D}^{2} / \lambda\right)$ so that near field effects do not alter the reference path loss. The reference distance should also be chosen to be about an order of magnitude less than the minimum T-R separation of measured data in order to prevent close-in data points that do not obey free space propagation from unfairly biasing the statistics. The reference path loss PL( $\left.d_{0}\right)$ is 
assumed to be due to free space propagation from the transmitter to $d_{0}$. Measurements show this is valid assumption to within $1 \mathrm{~dB}$ nominally [Rap89a].

$$
\mathrm{PL}\left(d_{0}\right)[\mathrm{dB}]=20 \times \log _{10}\left(\frac{4 \pi\left(d_{0}\right)}{\lambda}\right)[\mathrm{dB}]
$$

Path loss is often modeled as a log-normal distribution about the mean power law described by (2.3-2) [Cox84]. That is, $P L(d)=\overline{P L}(d)+X_{\sigma}$ where $X_{\sigma}$ is a zero-mean lognormally distributed (normal in $\mathrm{dB}$ ) random variable with standard deviation $\sigma$ in $\mathrm{dB}$. In [Haw90], [Haw91], [Sei91a], [Te191], [Sei92a], linear regression was used to compute values of the path loss exponent $n$ and the standard deviation $\sigma$ in $\mathrm{dB}$ about the best fit mean power law model in a minimum mean square error (MMSE) sense for measured path loss data [Dix83].

A statistical distance-dependent path loss model is useful for understanding the propagation of radio waves in buildings. However, exhaustive measurements were required to obtain the data to determine the appropriate parameters for the models for these particular buildings. In addition, the mean path loss exponents can vary from less than two to greater than six depending upon the specific environment (see Section 3.1 Statistical Path Loss Models). Models which allow a system designer to predict path loss contours for all types of buildings without measurements would be extremely valuable.

\subsection{Time-Dispersive Multipath Channel Parameters}

The mean excess delay, rms delay spread, delay interval, and excess delay spread are time-dispersive channel parameters that can be determined from a power delay profile Figure 2.4-1 shows a typical multipath power delay profile. The transmitter-receiver separation, path loss, rms delay spread, and receiver threshold are indicated in the figure. A receiver threshold is used to distinguish multipath signal from noise, and data points below this threshold are not used in computation of the channel parameters. 


\section{Measurement Location : Norris 1 F}

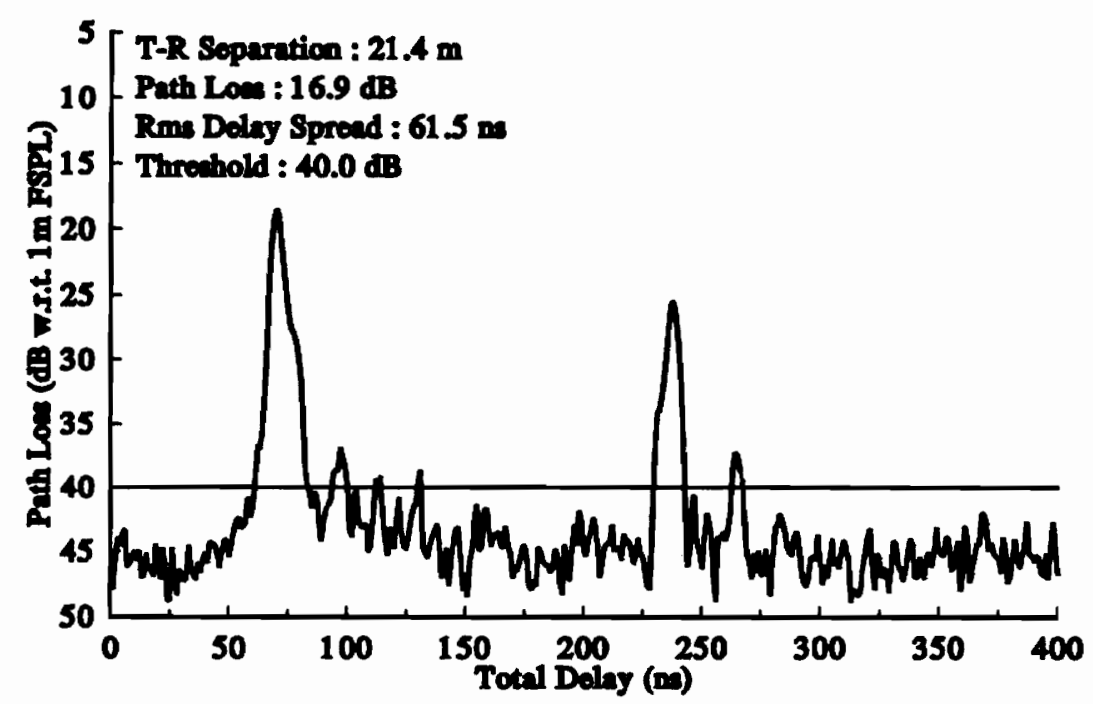

FIGURE 2.4-1. Measured power delay profile and statistical parameters used to describe the measurements.

\subsubsection{Mean Excess Delay}

The time dispersive properties of wide band multipath channels are grossly quantified by their mean excess delay $(\bar{\tau})$ and rms delay spread $\left(\sigma_{\tau}\right)$ [Dev86], [Dev87a], [Bul89], [Pah89], [Rap89b], [Dev90b], [Rap90a], [Sei91b] (and many others). The mean excess delay is the first moment of the power delay profile and is defined to be

$$
\bar{\tau}=\frac{\sum_{k} \alpha_{k}^{2} \tau_{k}}{\mathrm{G}_{\mathrm{r}}} \text { where } \mathrm{G}_{\mathrm{r}}=\sum_{k} \alpha_{k}^{2} .
$$

The change in the mean excess delay as a mobile terminal moves is called time delay jitter. Time delay jitter in indoor multipath channels has been measured in [Dev87b], [Rap88b]. Work in [Chu87b] shows that time delay jitter can cause bit errors due to imperfect timing recovery. The tracking error is proportional to the time delay jitter. The variables used to describe the time dispersive multipath channel parameters are given in Table 2.4-1. 


\section{TABLE 2.4-1: Summary of variables used in the calculation of mean excess delay} and rms delay spread.

\begin{tabular}{|c|c|}
\hline Varible & Becription (uitis) \\
\hline$\tau_{\mathbf{k}}$ & Time delay of $\mathrm{k}^{\text {th }}$ multipath component [seconds] \\
\hline$\alpha_{k}^{2}$ & Power of $\mathbf{k}^{\text {th }}$ multipath component [Watts] \\
\hline $\mathrm{G}_{\mathbf{r}}$ & Received power in power delay profile [Watts] \\
\hline $\bar{\tau}$ & Mean excess delay [seconds] \\
\hline$\overline{\tau^{2}}$ & Mean squared excess delay [seconds ${ }^{2}$ ] \\
\hline$\sigma_{\tau}$ & Rms delay spread [seconds] \\
\hline$t_{0}$ & Excess delay of first arriving multipath component [seconds] \\
\hline$\tau_{\mathrm{x}}$ & $\begin{array}{l}\text { Excess delay of last arriving component within } \mathrm{X} \mathrm{dB} \text { of maximum } \\
\text { received component [seconds] }\end{array}$ \\
\hline
\end{tabular}

\subsubsection{RMS Delay Spread}

The rms delay spread is the square root of the second central moment of the power delay profile and is defined to be

$$
\sigma_{\tau}=\sqrt{\tau^{2}-\bar{\tau}^{2}} \text { where } \overline{\tau^{2}}=\frac{\sum_{k} \alpha_{k}^{2} \tau_{k}^{2}}{\mathrm{G}_{\mathrm{r}}}
$$

In (2.4-1) and (2.4-2), the delays of each profile are measured relative to a first detectable signal arriving at $\tau_{0}=0$. The term $G_{r}$ in (2.4-1) and (2.4-2) is a relative measure of received power for a given profile snapshot.

It is important to note that here the rms delay spread and path loss are defined to be due to a single power delay profile which is the time average of consecutive impulse response measurements collected and averaged over time. Thus, new rms delay spread values are received every time a snapshot is made so that each measured rms delay spread 
value is representative of the local channel behavior. Originally, rms delay spread was computed for a set of power delay profiles averaged over time and distance [Cox73].

The rms delay spread is an important parameter in the determination of the bandwidth capacity of a radio channel [Jak74]. Recall that the power delay profile and frequency response measurements are Fourier Transform duals of each other. The rms delay spread and the coherence bandwidth are inversely proportional to each other. The delay spread is a natural phenomenon caused by multiple time-delayed scattered paths in the radio channel. The coherence bandwidth is a defined relation derived from the delay spread that quantifies the similarity of the channel frequency response at different frequencies [Lee89]. If the coherence bandwidth is defined as the bandwidth over which the frequency correlation function is above 0.9 , then the coherence bandwidth is approximately [Lee89]

$$
B_{c} \approx \frac{1}{50 \sigma_{\tau}}
$$

If the definition is extended so that the frequency correlation function is above 0.5 , then the coherence bandwidth is approximately

$$
B_{c} \approx \frac{1}{5 \sigma_{\tau}}
$$

Work in [Mor92] has shown a linear relationship between coherence bandwidth and rms delay spread. Signals with bandwidth greater than the coherence bandwidth suffer from intersymbol interference. Such channels are called frequency-selective channels because different frequencies fade differently. Adaptive equalizers or antenna diversity systems can be used to flatten the channel amplitude and phase response when the signal bandwidth is greater than the coherence bandwidth. Even with equalization and antenna diversity, the intersymbol interference causes an irreducible bit error floor that is proportional to the delay spread [Be163], [Chu87a]. That is, the minimum bit error rate will increase as the ratio of delay spread to bit period increases, independent of signal-to-noise ratio. 


\subsubsection{COST 207 Delay Interval}

The European Community COST 207 Project defines the delay interval to be the time window that contains a certain portion of the energy [deW88]. In [Rae91], the same term is defined to be the delay window. A combined term will be used here. The delay interval window is computed at the center of the power delay profile so that half of the energy outside the window is received before the start of the window, and half of the energy is received after the end of the window. Figure 2.4-2 shows a measured power delay profile and the Delay Interval for $90 \%$ of the received energy inside the window. Notice that half of the energy outside the window arrives before the window and half arrives after the window. This parameter is a useful measure of the time dispersion of the power delay profile.

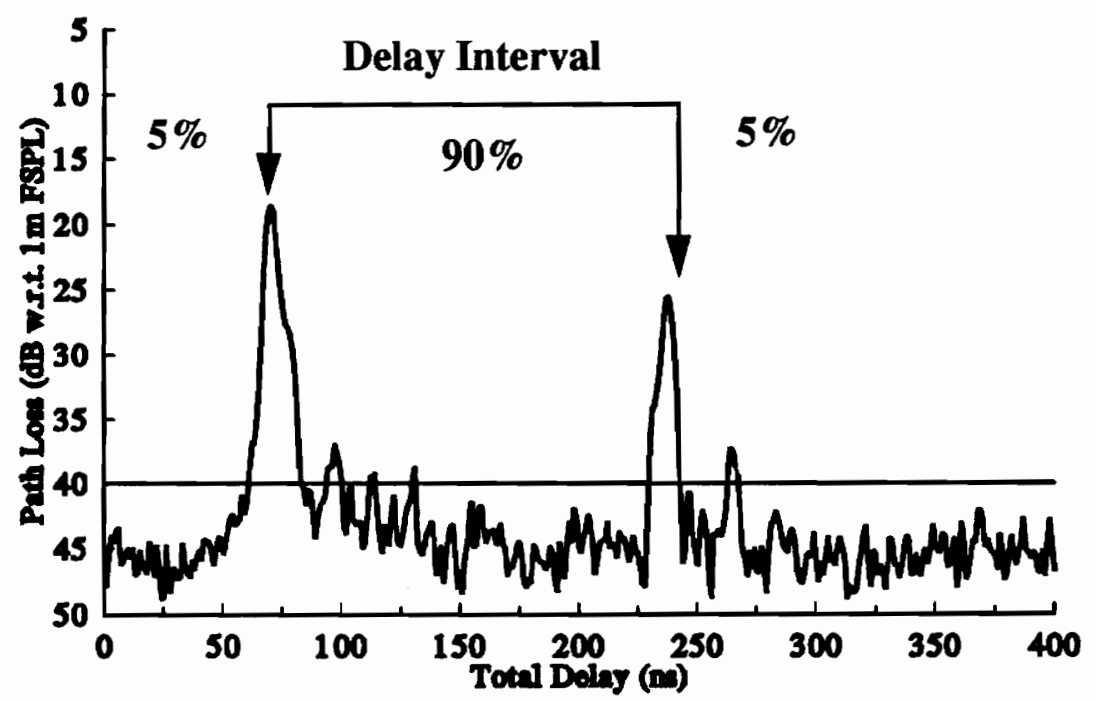

FIGURE 2.4-2. Measured power delay profile and COST 207 delay interval for $90 \%$ of the received power.

\subsubsection{Excess Delay Spread (X dB)}

The excess delay spread of a channel is defined as $\tau_{\mathbf{x}}-\tau_{0}$ where $\tau_{0}$ is the first arriving signal and $\tau_{\mathbf{x}}$ is the maximum delay at which a multipath component is within $\mathrm{X} \mathrm{dB}$ of the strongest arriving multipath signal. An example is shown in Figure 2.4-3 for multipath components within $10 \mathrm{~dB}$ of the maximum. The excess delay spread defines the extent of 
the multipath that can be deemed significant in the design and analysis of equalization for TDMA systems and rake reception for CDMA systems.

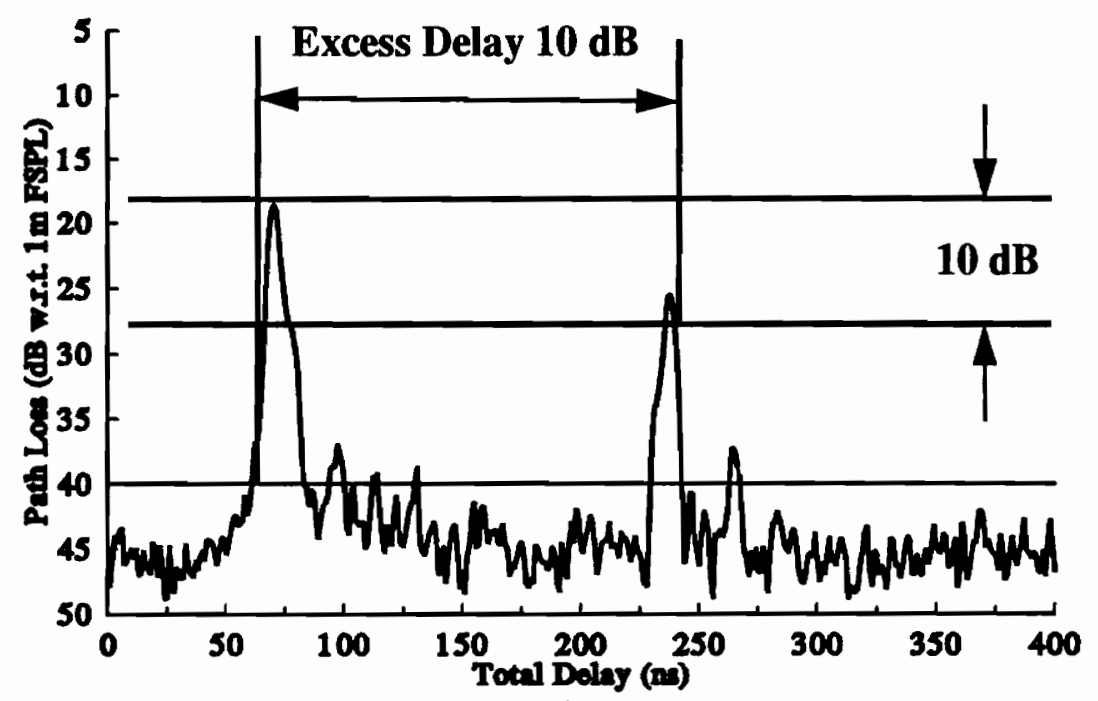

FIGURE 2.4-3. Measured power delay profile and excess delay $10 \mathrm{~dB}$.

\subsection{Multipath Propagation Measurements}

This section discusses multipath propagation channel measurements. Both wide band and narrow band characterization methods are presented. Data processing techniques that allow channel parameters such as path loss and delay spread to be determined from the measured data are given.

In [Rap89b], it was shown that CW measurements averaged over space and wide band measurements averaged over time give equivalent path loss results when multipath component phases are independent and identically uniformly distributed over $[0,2 \pi)$ or when multipath component amplitudes are uncorrelated. Hence, a simple CW measurement system ( $\mathrm{B}_{\mathrm{RF}} \sim 15 \mathrm{kHz}$ ) may be used to give path loss results that may be applied to wide band channels $\left(B_{R F}>B_{C}\right)$ as well when the wide band channel is averaged over space. This can be useful when there is not enough spectrum available for propagation experiments in a particular frequency band. When the data rate of a proposed communications system is less than the coherence bandwidth, a narrow band channel characterization 
is sufficient to determine system performance. Path loss and fading statistics are of primary concern. When the data rate is larger than the coherence bandwidth, the structure of the channel impulse response must be known to determine the extent of intersymbol interference.

\subsubsection{CW Measurements and Data Processing}

Narrow band propagation measurements are useful for determining average path loss coverage areas for proposed systems, and for acquiring a large amount of path loss data in a relatively short period of time. Also, since thermal noise is proportional to receiver bandwidth, narrow band system measurement dynamic ranges are greater than direct pulse wide band systems, and measurements are less susceptible to in-band interference. This makes it possible to easily characterize commercial frequency bands. The block diagram of a simple CW measurement system used in [Sei91a], [Te191], [Sei92a], is shown in Figure 2.5-1. A CW signal at a carrier frequency is generated, amplified, and transmitted. The received signal strength is continuously monitored and stored on disk. The modified commercial receiver can instantaneously measure signals between $0 \mathrm{dBm}$ and $-91 \mathrm{dBm}$ over a $15 \mathrm{kHz}$ bandwidth at $915 \mathrm{MHz}$ [Lui90]. Typically, omni-directional antennas such as discones [Rap88a] and quarter-wave monopoles are used so that the antenna pattern has little influence on the path loss measurement.

In [Sei91a], [Te191], [Sei92a], locations which are potential candidates for future microcellular base stations, such as centrally located areas, and perimeter areas within a wing of a building were chosen for most transmitter sites. For each transmitter location, the mobile receiver thoroughly canvassed the building at transmitter-receiver (T-R) separations that ranged between $1.5 \mathrm{~m}$ and $90 \mathrm{~m}$. During each measurement, the mobile receiver moved at constant walking velocity along a straight path which varied in length between 2.4 and 60 meters, depending on surroundings. Measurement personnel continuously recorded the mobile's position on logsheets so that site-specific propagation models could be developed from the data. Propagation studies that examine the effects of carrier frequency, antenna height, antenna pattern, antenna polarization, and obstructing objects 


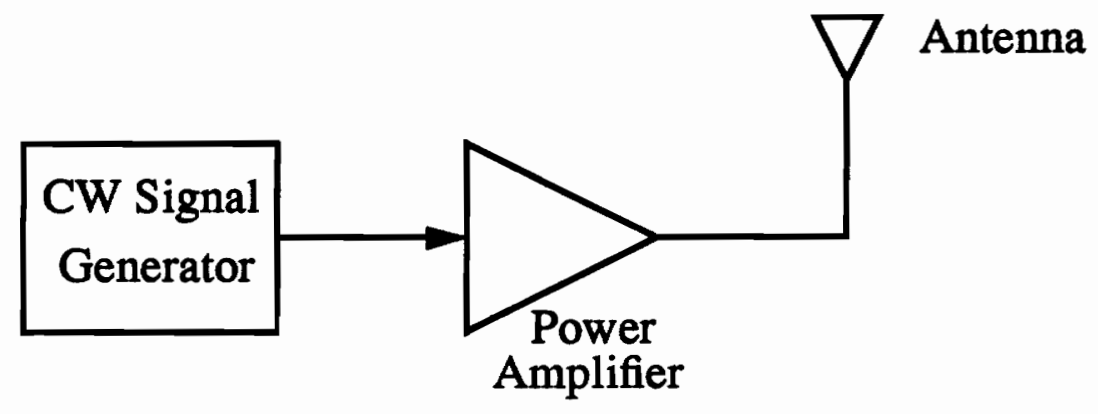

Transmitter

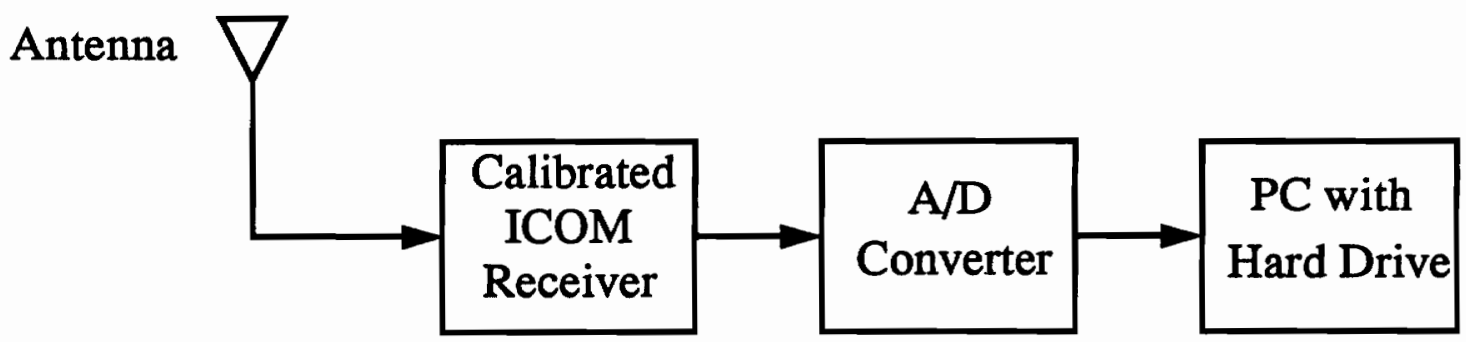

\section{Receiver}

FIGURE 2.5-1. Block diagram of a CW measurement system.

like floors and partitions [Haw91], [Sei91a], [Sei92a] on path loss and/or fading statistics can easily utilize the measurement system shown in Figure 2.5-1.

Figure 2.5-2 shows a typical measurement run where the receiver was moved along a straight path in an office building. The abscissa represents the T-R separation. The median signal strength is computed as a spatial average over several wavelengths of the instantaneous received signal [Uri91], and is considered a discrete measurement location to develop path loss models and contours. In [Sei92a], the median signal strength over a distance of $20 \lambda(6.56 \mathrm{~m})$ was computed at $20 \lambda$ intervals for each measurement run. A $20 \lambda$ distance was chosen so that the fast fading of the envelope caused by multipath would not influence the large-scale path loss for a given measurement track. Discrete path loss measurements can be combined statistically to determine mean path loss exponents (defined in Section 2.3 Path Loss) for the measured data. The data may also be related to the site-spe- 
cific measurement location to develop path loss models that predict propagation more accurately and contour plots that show shadow regions for a given transmitter location.

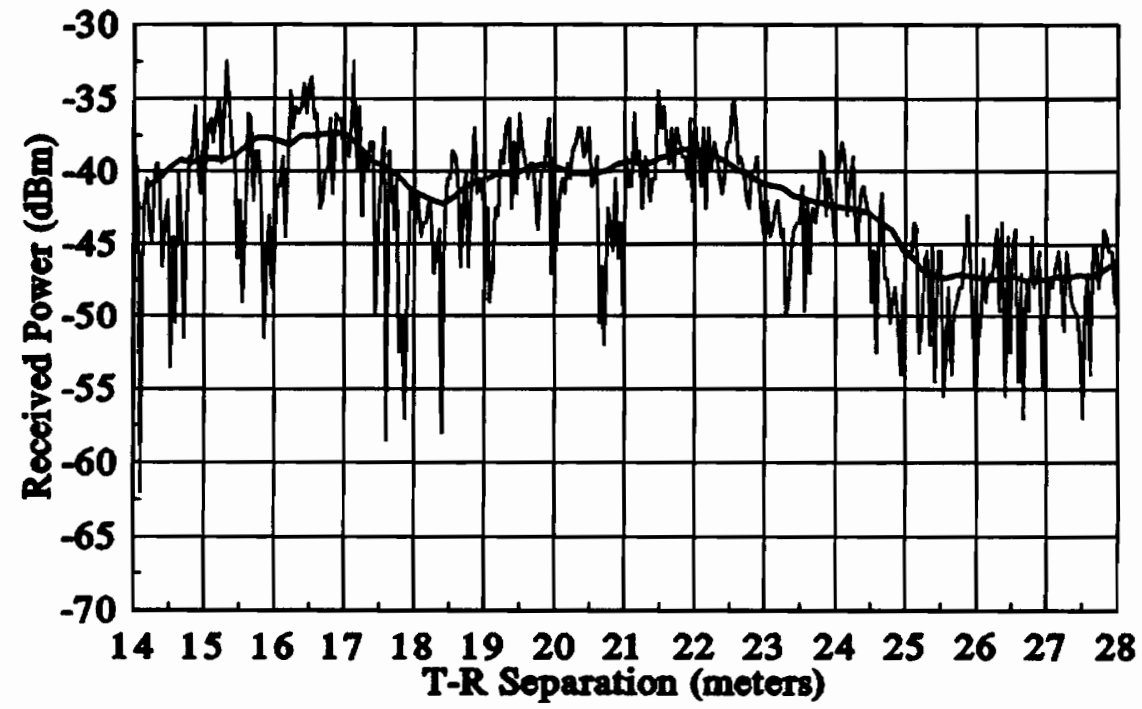

FIGURE 2.5-2. Typical CW fading run. The moving average over a $20 \lambda$ distance is also shown in the figure.

\subsubsection{Direct Pulse Measurements and Data Processing}

For direct pulse measurements, a finite time duration pulse of width $T_{b b}$ is convolved with a CW carrier wave (recall Figure 2.3-1). The resultant signal is amplified and transmitted. The block diagram of a typical pulse measurement system is shown in Figure 2.5-3 and Figure 2.5-4. The receiver is a multi-stage tuned RF receiver. Consider the first row of the block diagram in Figure 2.5-4. The first variable attenuator prevents the amplifier from becoming saturated when the receiver is close to the transmitter. The RF bandpass filter prevents spurious signals at frequencies outside the measurement band from saturating the wide band (0-4.2 GHz in [Haw91], [Te191]) amplifier. The second variable attenuator adjusts the gain of the receiver so that there is nearly full-scale deflection on the oscilloscope screen. On the second row of the block diagram, a second amplifier-filter chain is used so that the receiver is noise-limited rather than gain-limited. A noise-limited receiver generally has a greater dynamic range than a gain-limited receiver since the minimum detectable signal is determined at the input of the receiver, and is not limited by the inability of the receiver to amplify the signal level into the dynamic range of the detector. 


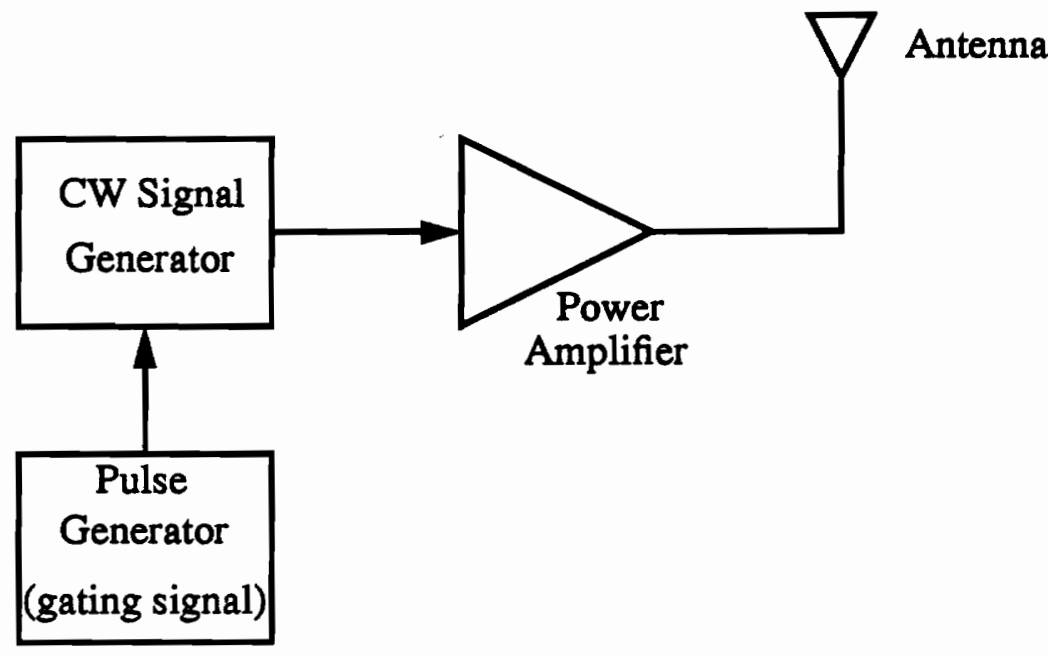

\section{Pulse Transmitter}

FIGURE 2.5-3. Block diagram of a typical pulsed transmitter.

A power coupler is used so that the received RF spectrum can be monitored for in-band and adjacent band interference that may pass through the filters. In the third row, an RF-tobaseband detector strips off the RF carrier and outputs a baseband video signal that is the squared envelope of the RF signal. A matching attenuator (typically $3 \mathrm{~dB}$ ) is used to reduce the impedance mismatch between the detector and the digital oscilloscope in order to preserve pulse shape. A digital oscilloscope records the squared magnitude of the impulse response (power delay profile). A personal computer controls the oscilloscope and stores the data for processing. The receiver is calibrated by inputting a known signal level into the front-end and recording the oscilloscope deflection as the input level is varied over the receiver dynamic range. 

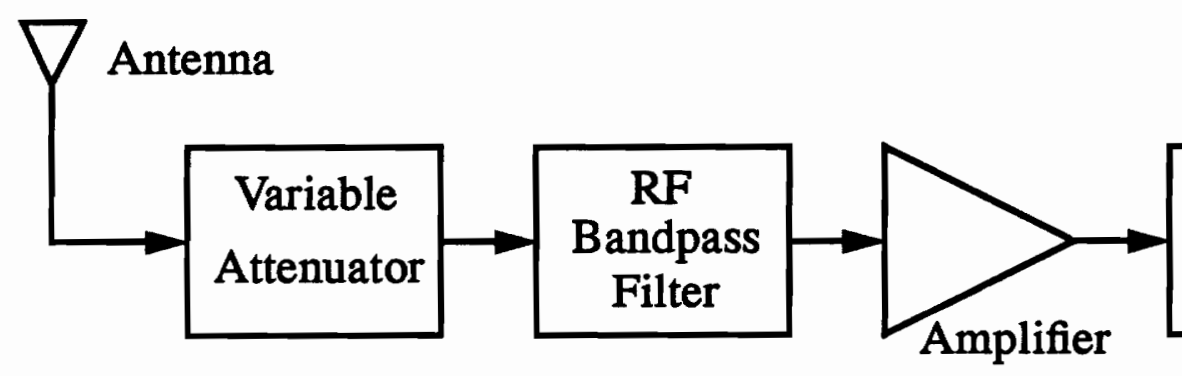

\section{Variable \\ Attenuator}

Amplifier
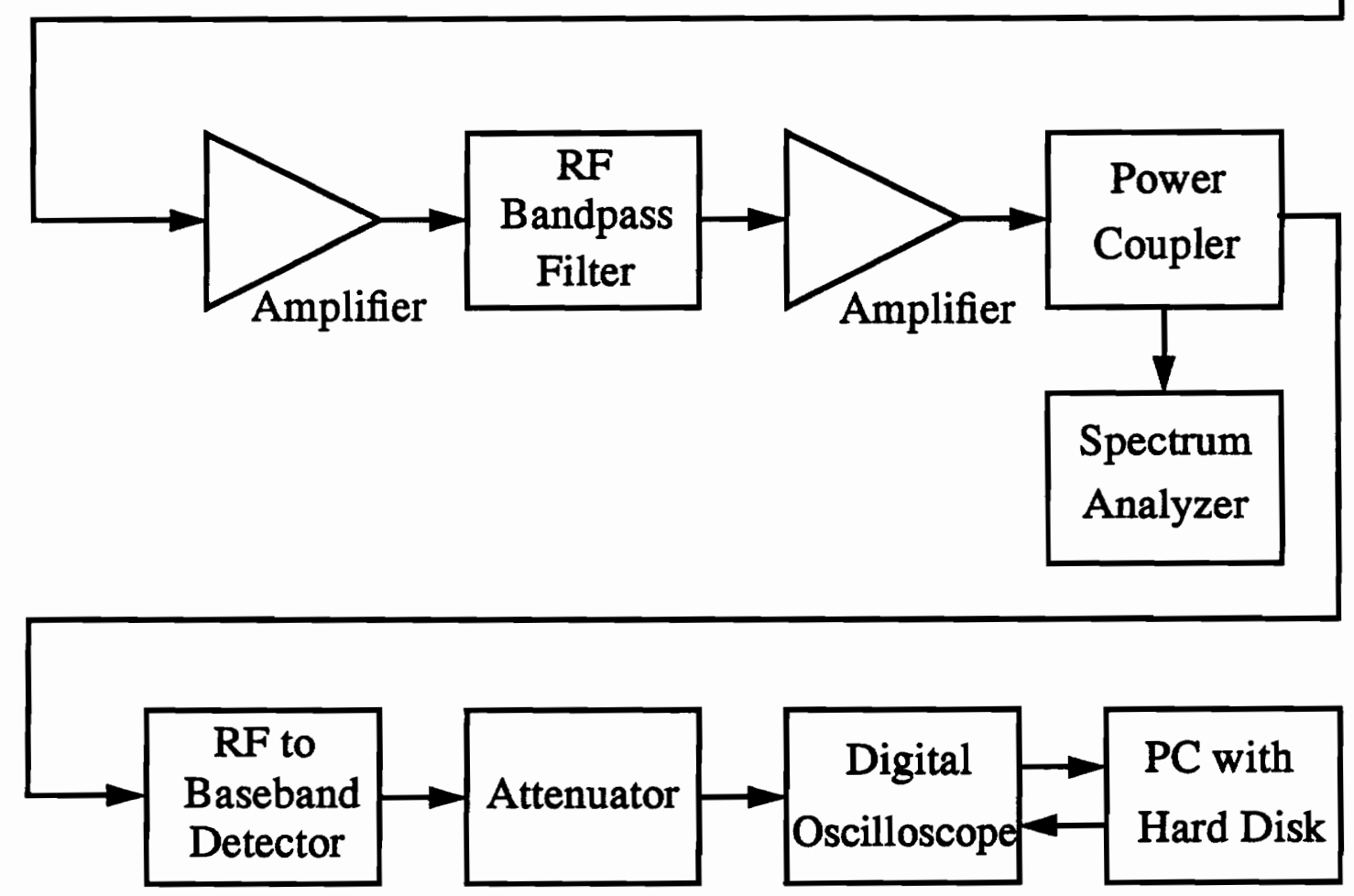

\section{Pulse Receiver}

FIGURE 2.5-4. Block diagram of a typical wide band direct pulse receiver. The receiver is a tuned $R F$ receiver. 


\section{TABLE 2.5-1: Table of variables and descriptions used to describe the computation of path loss from a wide band power delay profile.}

\begin{tabular}{|c|c|}
\hline Varable & Description fuints] \\
\hline$\alpha_{k}^{2}$ & Received power [mW] \\
\hline$G_{r}=\sum \alpha_{k}^{2}$ & Received power at antenna output [mW] \\
\hline PL & Channel path loss [dB] \\
\hline $\mathbf{P}_{\mathbf{T}}$ & Peak transmitter power $[\mathrm{dBm}]$ \\
\hline $\mathrm{G}_{\mathbf{T}}$ & Transmitter antenna gain [dBi] \\
\hline $\mathrm{G}_{\mathrm{R}}$ & Receiver antenna gain [dBi] \\
\hline $\mathbf{P}_{\mathrm{CAL}}$ & Peak power of a calibration measurement $[\mathrm{dBm}]$ \\
\hline $\mathrm{G}_{\mathrm{CAL}}$ & Received integrated power of a calibration run [mW] \\
\hline$\Delta \tau_{r}$ & Sample interval of a measurement [seconds] \\
\hline$\Delta \tau_{C A L}$ & Sample interval of a calibration measurement [seconds] \\
\hline$T_{b b}$ & Pulse width [seconds] \\
\hline
\end{tabular}

Table 2.5-1 summarizes the variables used in wide band data processing of a power delay profile. In order to compute the wide band path loss, it is necessary to compute the power received by integrating the area under the power delay profile. The term $G_{r}$ is the integrated power in a given power delay profile. For a calibration run where only one pulse of width $T_{b b}$ is present, the total integrated power is assigned the known input power, $P_{C A L}$. The term $G_{C A L}$ converts the total integrated power to an equivalent peak power in a pulse of width $\mathrm{T}_{\mathrm{bb}}$. Path loss is referred to isotropic antennas.

Through calibration of the receiver system, the path gain factors $\alpha_{k}^{2}$ are the actual received power in $\mathrm{mW}$ determined from the oscilloscope display voltage. The attenuator setting of the receiver is included in calibration of the power delay profile. Let PL denote the channel path loss, $P_{T}$ denote the peak transmitter power, $G_{T}$ denote the transmitter antenna gain, and $G_{R}$ denote the receiver antenna gain. Then, path loss is given by [Rap89b]

$$
P L(\mathrm{~dB})=P_{T}(\mathrm{dBm})+G_{T}(\mathrm{dBi})+G_{R}(\mathrm{dBi})-P_{R}(\mathrm{dBm})
$$




$$
P_{R}(\mathrm{dBm})=P_{C A L}(\mathrm{dBm})+10 \times \log _{10}\left[\frac{G_{r} \Delta \tau_{r}}{G_{C A L} \Delta \tau_{C A L}}\right]
$$

It is important to point out the importance of receiver sensitivity and noise threshold in interpretation of measured channel parameters. Generally, a noise threshold is applied to power delay profiles in order to distinguish between actual multipath components and receiver noise. The selection of this noise threshold is critical in determining channel parameters such as rms delay spread which is defined in the following section. This is similar to the radar probability of detection vs. probability of false alarm problem. If noise is detected as a multipath signal, the channel parameters could be grossly overestimated. If a multipath component is not detected, system simulation studies such as bit error rate and equalizer performance requirements could be underestimated. Experience has shown that it is necessary to determine an appropriate threshold level for power delay profiles to avoid the inclusion of profiles that had a lost trigger or were corrupted with impulsive noise [Rap90a], [Sei91b], [Te191]. Although attempts have been made to develop an algorithm to determine the threshold level [Rap90a], [Haw91], it is common practice for a trained operator determine the threshold level based on visual inspection of each power delay profile [Te191]. As a minimum requirement, visual inspection should be used to verify that the automatic procedure is working correctly. An example of a threshold chosen upon visual inspection is given in Figure 2.4-1.

\subsubsection{Spread Spectrum Measurements}

Spread spectrum measurement systems are an alternate method of measuring the wide band power delay profile. The main advantage of this spread spectrum receiver system is that wide bandwidth impulse response estimates may be measured with a dynamic range that is comparable to that of narrow band measurement systems. This is accomplished by de-spreading the probing signal before detection by a narrow band receiver. The narrow bandwidth of the detection circuitry provides the increased dynamic measurement range. Another advantage is that spread spectrum measurement systems provide greater dynamic display measurement range than direct pulse measurements. That is, a spread spectrum measurement system can detect multipath components that have ampli- 
tudes further below the amplitude of the maximum multipath component detected. The dynamic display range is limited by the maximum cross-correlation of the spreading code. For a code length of 2047 chips, the maximum dynamic display range is $33 \mathrm{~dB}$. Allowing for some noise, a practical display range is about $30 \mathrm{~dB}$. Longer chip sequences can provide larger dynamic display ranges. For pulse systems, the dynamic display range is limited by the number of quantized levels on a digital oscilloscope. Theoretically this value is $30 \mathrm{~dB}$, however, amplifier noise usually reduces this to around $20 \mathrm{~dB}$ (see Figure 2.4-1).

This section describes a continuous spread spectrum channel sounder. The chip rate is $240 \mathrm{MHz}$, allowing a time resolution of about $8 \mathrm{~ns}$. The transmitter consists of 4 parts: a carrier frequency source, a chip clock frequency source, a BPSK modulator, and a power amplifier. The chip clock frequency is generated by a TV tuner at $240 \mathrm{MHz}$. The

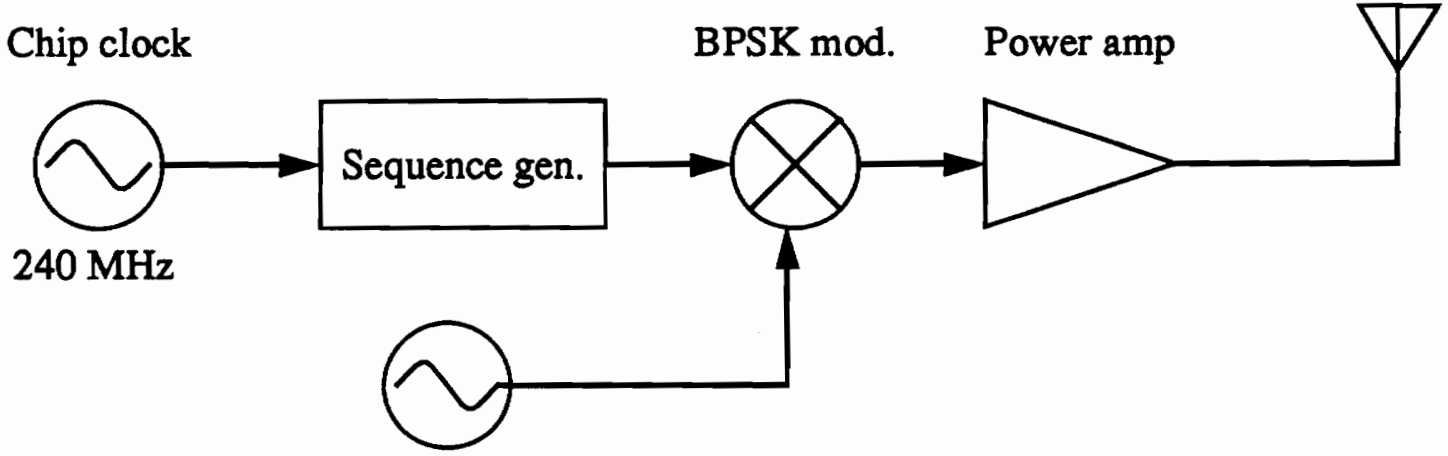

Carrier frequency

FIGURE 2.5-5. Continuous spread spectrum transmitter

spreading sequence and carrier frequency are mixed, amplified and transmitted. The receiver consists of a low-noise amplifier and an identical sequence running $40 \mathrm{kHz}$ slower than the one at the transmitter. This difference in clock frequencies causes the relative phase of the two sequences to "slide" constantly. This sliding effect is the key to the channel sounder's operation. If the channel were ideal, there would only be one point of maximum correlation. This maximum correlation would occur while the sequence generators were at the same point in the sequence. A spread spectrum communications system would lock the sequence at this point. However, since propagation measurements are interested in the channel impulse response, the sequences are allowed to continue sliding 
past the point of maximum correlation in order to detect all peaks that occur due to time delayed multipath components. This amplitude variation with time is captured by a spectrum analyzer set to zero span. The spectrum analyzer output is displayed on a digital oscilloscope and the signal is stored on a computer for data processing. The data stored on disk are processed in the same manner as the 'raw' oscilloscope data recorded with the direct pulse measurement system except that the time samples must be converted to actual time. This is necessary because the oscilloscope records the rate that the codes slip past one another and not the actual time of each multipath component.

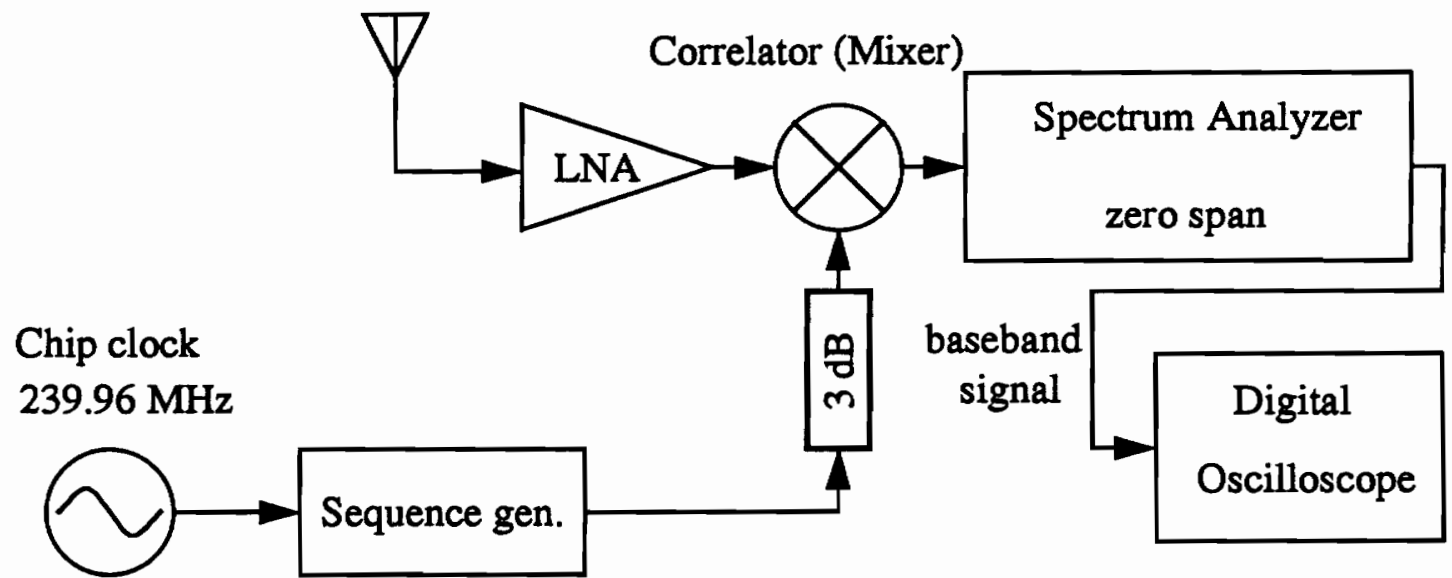

FIGURE 2.5-6. Spread Spectrum receiver.

Typically the minimum receivable signal is about $-110 \mathrm{dBm}$ at $4 \mathrm{GHz}$. Measurements with a pulse receiver in [Te191] had a minimum detectable signal of $-80 \mathrm{dBm}$ at 915 MHz. The dynamic range of the measurement system is limited by the low noise amplifier at the front end.

\subsection{Summary}

This chapter began with a description of multipath propagation and its effect on transmitted signals as a function of signal bandwidth. Narrow band signals were shown to have rapid amplitude fluctuations as a function of position or frequency. Wider bandwidth signals do not exhibit these rapid fluctuations, and individual multipath components can be distinguished with a resolution inversely proportional to the bandwidth. A discrete 
wide band channel model was developed. Path loss and multipath channel parameters that quantify the time-dispersive nature of the propagation channel were then defined. Methods for measuring the mobile and portable radio propagation environment and determining multipath channel parameters from the measured data were presented. The following chapter presents propagation models based on in-building propagation measurements in a variety of different buildings. 


\section{Propagation Models Based on Measurements}

\subsection{Overview}

Much work has been done to characterize radio propagation in buildings in the 800 $\mathrm{MHz}$ to $5.8 \mathrm{GHz}$ frequency bands [Ale83a], [Ale83b], [Cox84], [Cox85], [Dev86], [Hor86], [Bul87], [Dev87a], [Sal87], [Arn89], [Bul89], [Chi89], [Dev89], [Owe89], [Pah89], [Pat87], [Rap89a], [Rap89b], [Dev90a], [Dev90b], [Haw90], [Dev91a], [Dev91b], [Te191], [Lib92], [Tod92]. A comprehensive literature survey of radio propagation into and within buildings is given in [Mo191]. Previous work has focused on measurements and statistical characterization of the radio frequency environment. Models that exploit specific building features are given in [Mot88], [Laf90], [Rap91a], [Sei91a], [Sei92a]. The models in [Sei91a] and [Sei92a] are major contributions of this dissertation. This chapter presents a survey of the state of the art of statistical measurement-based propagation models in many different building environments. Models for path loss as a function of distance and median and maximum rms delay spreads are presented for different buildings. The effects of frequency and building type on path loss and delay spread are discussed. A statistical multipath channel model simulator that uses the statistical results of propagation measurements in a wide variety of buildings, SIRCIM, is described. This chapter concludes with promising site-specific path loss models in [Sei91a] and [Sei92a] that account for the shadowing caused by floors, walls, office partitions, and common objects found in many different types of buildings. In fact, the good agreement of these site-specific models with measurements led to the development of the ray tracing propagation model presented in Chapter 5 which is the primary contribution of this Ph.D. dissertation.

\subsection{Statistical Path Loss Models}

The variation of path loss throughout a building impacts coverage areas and base station layout design. Work in [Ber89] showed how path loss characteristics affect the distance between transmitter and receiver when a call is initiated. Table 3.1-1 provides a summary of path loss exponents and rms delay spread in a variety of buildings as gathered 
from the literature. Blank entries indicate that these parameters were not determined from the measurements. The different types of buildings in Table 3.1-1 are described below.

Open-plan buildings typically have large open areas with a high ceiling. Obstructing objects do not span all the way from the floor to the ceiling. Factories are commonly open-plan buildings where metal machinery, inventory, and exterior walls comprise the majority of scatterers. A soft-partitioned environment describes a typical multi-story office building with large open areas that are partitioned into office cubicles by $\sim 1.5 \mathrm{~m}$ high movable metal frame cloth-covered dividers.

A hard-partitioned building is a typical multi-story office building with many internal partitions constructed of reinforced concrete and/or dry wall supported by metal or wooden studs. Corridors are typically 1.8 to 3.9 meters wide. Walls that partition offices from corridors are constructed from the floor to the ceiling. For the purposes of classification of building type in Table 3.1-1, an urban high-rise is a hard-partitioned building located in a dense urban environment surrounded by many other tall buildings. The other hard-partitioned buildings are less than ten stories tall and are not located in a clustered downtown area. The urban high-rise building in [Te191] was twenty-six stories tall in the downtown financial district of San Francisco.

The sports arena in [Haw91] was a typical college basketball arena that seats about six thousand. There was a large open area and a concourse level that surrounded the open area. There were many openings between the open area and the concourse level. The typical grocery store in [Sei92a] had an open-plan shopping area that consisted of metal shelves with aisles between the shelves. The retail store was divided into several departments separated by soft-partitions. The residences in [Dev86] were a typical wood frame house and wood frame apartment building. 
TABLE 3.1-1: Summary of path loss and delay spread measured in a variety of buildings.

\begin{tabular}{|c|c|c|c|c|c|c|}
\hline Builing (s) & Rerrenge & (meq, & 4 & $\left(\frac{0}{(d B)}\right.$ & 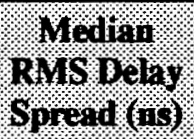 & $\begin{array}{l}\text { Mariming } \\
\text { RMS beiay } \\
\text { Spread (as) }\end{array}$ \\
\hline Open-Plan Factories & [Rap89a] & 1300 & 2.2 & 7.9 & & \\
\hline Open-Plan Factories & [Rap89b] & 1300 & 2.2 & & 100 & 300 \\
\hline Open-Plan Factories & [Pah89] & 910 & $1.4-3.3$ & & $15-50$ & $40-150$ \\
\hline Open-Plan Factory B & [Haw91] & 1300 & 2.0 & 3.7 & 70 & 130 \\
\hline Open-Plan Factory B & [Haw91] & 4000 & 2.1 & 4.0 & 70 & 130 \\
\hline Open-Plan Factory C & [Haw91] & 1300 & 2.4 & 9.2 & 40 & 120 \\
\hline Open-Plan Factory C & [Haw91] & 4000 & 2.1 & 9.7 & 40 & 120 \\
\hline $\begin{array}{l}\text { Suburban Office Building } \\
\text { - Open-Plan }\end{array}$ & [Tel91] & 915 & 2.4 & 9.6 & 74 & 139 \\
\hline $\begin{array}{l}\text { Suburban Office Building } \\
\text { - Open-Plan }\end{array}$ & [Te191] & 1900 & 2.6 & 14.1 & 94 & 440 \\
\hline $\begin{array}{l}\text { Suburban Office Building } \\
\text { - Soft Partitioned }\end{array}$ & [Te191] & 915 & 2.8 & 14.2 & 67 & 214 \\
\hline $\begin{array}{l}\text { Suburban Office Building } \\
\text { - Saft Partitioned }\end{array}$ & [Tel91] & 1900 & 3.8 & 12.7 & 88 & 510 \\
\hline $\begin{array}{l}\text { Suburban Office Building } \\
\text { - Soft Partitioned : } 1\end{array}$ & [Sei92a] & 914 & 3.5 & 12.8 & & \\
\hline $\begin{array}{l}\text { Suburban Office Building } \\
\text { - Soft Partitioned : } 2\end{array}$ & [Sei92a] & 914 & 4.3 & 13.3 & & \\
\hline $\begin{array}{l}\text { Medium Office Building - } \\
\text { Hard Partitioned }\end{array}$ & [Sa187] & 1500 & $\sim 3$ & & 25 & 50 \\
\hline $\begin{array}{l}\text { Office Building - Hard } \\
\text { Partitioned }\end{array}$ & [Dev86] & 850 & & & & 321 \\
\hline $\begin{array}{l}\text { Large Office Building - } \\
\text { Hard Partitioned }\end{array}$ & [Dev87a] & 850 & & & 120 & 250 \\
\hline $\begin{array}{l}\text { Medium Office Building - } \\
\text { Hard Partitioned }\end{array}$ & [Dev87a] & 850 & & & 60 & 218 \\
\hline $\begin{array}{l}\text { Office Building - Hard } \\
\text { Partitioned : CRC }\end{array}$ & [Bul89] & 910 & & & 30 & $<70$ \\
\hline $\begin{array}{l}\text { Office Building - Hard } \\
\text { Partitioned : CRC }\end{array}$ & [Bul89] & 1700 & & & 29 & $<70$ \\
\hline $\begin{array}{l}\text { Office Building - Hard } \\
\text { Partitioned : CU }\end{array}$ & [Bul89] & 910 & & & 26 & $<70$ \\
\hline $\begin{array}{l}\text { Office Building - Hard } \\
\text { Partitioned : CU }\end{array}$ & [Bul89] & 1700 & & & 28 & $<70$ \\
\hline
\end{tabular}


TABLE 3.1-1: Summary of path loss and delay spread measured in a variety of buildings.

\begin{tabular}{|c|c|c|c|c|c|c|}
\hline Grilaring (s) & Rerronce & (nirgil) & 4 & 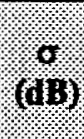 & 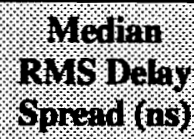 & 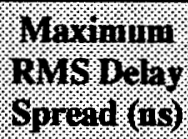 \\
\hline $\begin{array}{l}\text { Office Building - Hard } \\
\text { Partitioned }\end{array}$ & [Haw91] & 1300 & 3.4 & 14.1 & 25 & 40 \\
\hline $\begin{array}{l}\text { Office Building - Hard } \\
\text { Partitioned }\end{array}$ & [Haw91] & 4000 & 2.8 & 16.0 & 25 & 40 \\
\hline $\begin{array}{l}\text { Urban High-Rise Office } \\
\text { Building }\end{array}$ & [Te191] & 915 & 2.3 & 10.5 & 76 & 112 \\
\hline $\begin{array}{l}\text { Urban High-Rise Office } \\
\text { Building }\end{array}$ & [Tel91] & 1900 & 3.9 & 16.0 & 77 & 1470 \\
\hline Grocery Store & [Sei92a] & 914 & 1.8 & 5.2 & & \\
\hline Retail Store & [Sei92a] & 914 & 2.2 & 8.7 & & \\
\hline Sports Arena & [Haw91] & 1300 & 2.0 & 7.9 & 40 & 120 \\
\hline Sports Arena & [Haw91] & 4000 & 2.2 & 9.3 & 40 & 120 \\
\hline Residence - Apt. Bldg. & [Dev86] & 850 & & & & 422 \\
\hline Residence - House & [Dev86] & 850 & & & & 312 \\
\hline
\end{tabular}

Scatter plots of path loss vs. T-R separation for soft-partitioned office building measurements are given in Figure 3.1-1 and Figure 3.1-2. The dotted lines indicate the distance-dependent mean path loss model (equation (2.3-2)) for $n=1$ through $n=6$ and a 1 meter reference distance. The dashed line indicates the best mean path loss model in a MMSE sense for the data presented in the scatter plot. Different symbols are used to indicate data from different environments, and overall $n$ and $\sigma$ are given on the left side of each graph. Multi-floor measurements were possible in the two office buildings, and nearly all measurements had multiple obstructions such as concrete walls, windows, and soft partitions between the transmitter and receiver. From Figure 3.1-1, mean path loss increases with distance to the 3.54 power with a large standard deviation of $12.8 \mathrm{~dB}$ in office building 1 from [Sei92a]. The simple $d^{\mathbf{n}}$ path loss model in Figure 3.1-1 does not use site-specific information such as knowledge of office partitions or the number of floors between the transmitter and receiver. Transmission between more obstructions leads to higher path loss. 


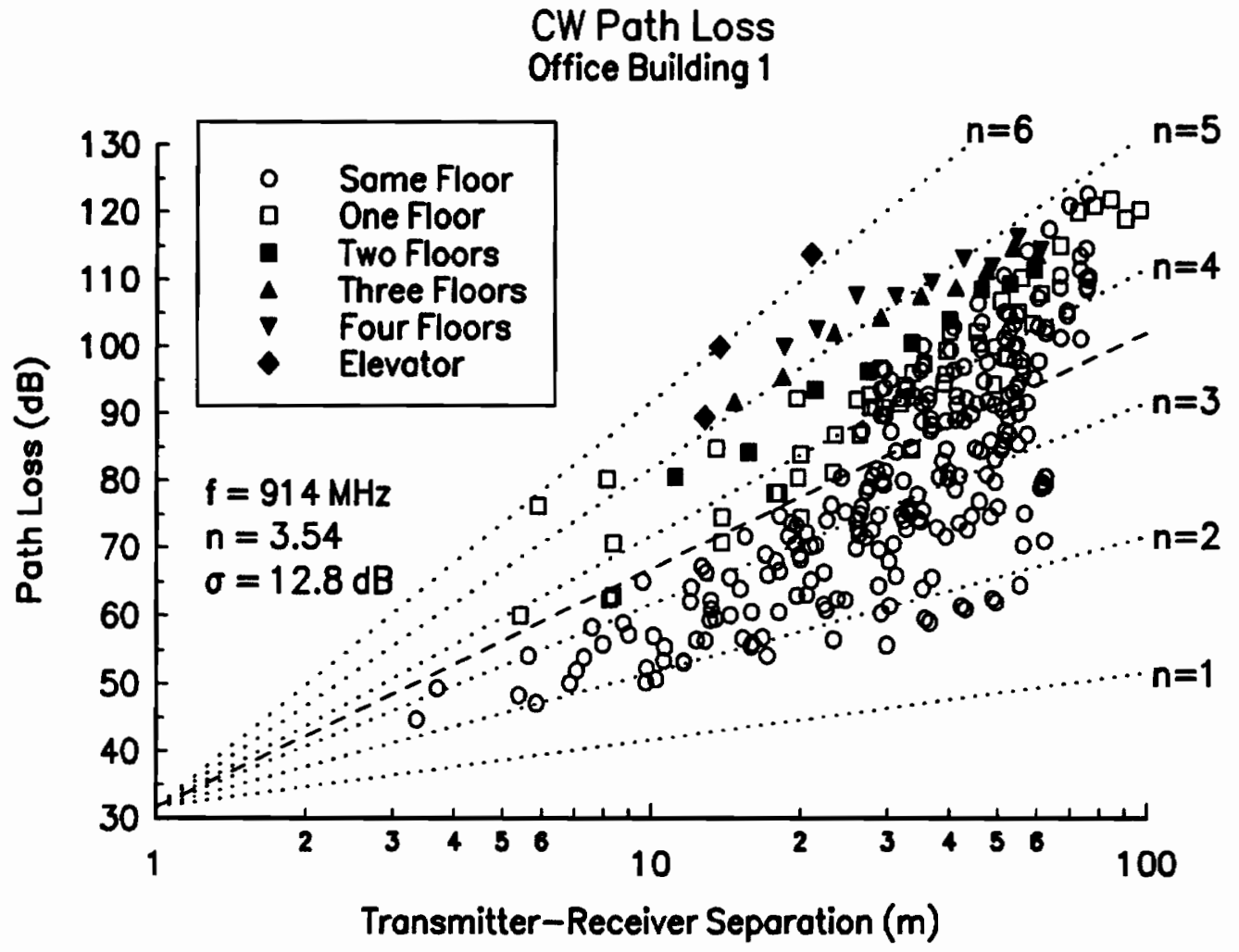

FIGURE 3.1-1. Scatter plot of CW path loss as a function of distance in office building 1 . The symbols indicate the number of floors between the transmitter and receiver (from [Sei92a]).

In office building 2 in [Sei92a], mean path loss increases with distance to the 4.33 power as shown in Figure 3.1-2. The number of floors between the transmitter and receiver can be seen to severely influence the path loss for a given T-R separation. Thus, the number of floors has an impact on the parameter $n$ in the path loss model, and should be quantified for accurate path loss prediction. Section 3.6 Site-Specific Path Loss Models presents mean path loss models for multi-floor environments.

In open-plan factory buildings, mean path loss exponents range between 1.4 and 3.3. The majority of values are between 2.0 and 2.4. Standard deviations are typically between $4.0 \mathrm{~dB}$ and $9.5 \mathrm{~dB}$. All measurements were conducted with the transmitter and receiver on the same floor. In [Te191], an open-plan office building had mean path loss exponents of 2.4 to 2.6 and larger standard deviations of 9.6 and $14.1 \mathrm{~dB}$. The larger vari- 
Office Building 2

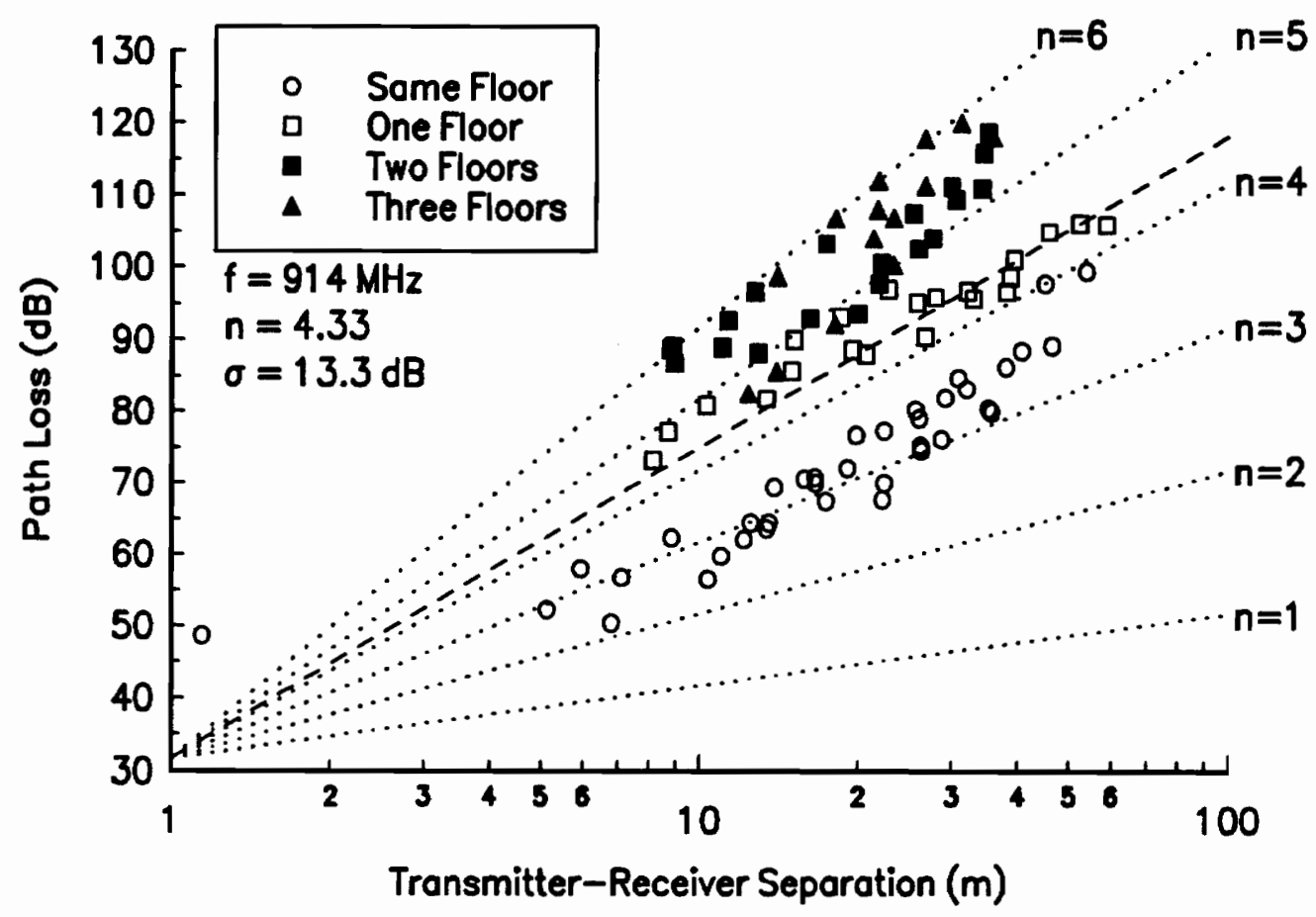

FIGURE 3.1-2. Scatter plot of $\mathbf{C W}$ path loss as a function of distance in office building 2. Notice the clustering of data as a function of the number of floor: between transmitter and receiver (from [Sei92a]).

ability was attributed to multiple floors between the transmitter and receiver for several of the measurements.

In soft-partitioned office buildings, mean path loss exponents range between 2.8 and 4.3. The standard deviations are between 12.7 and $14.2 \mathrm{~dB}$. due to multiple floors and obstructing soft-partitions between the transmitter and receiver. Site-specific models that attribute a certain amount of path loss to soft partitions and concrete walls are discussed in Section 3.6 Site-Specific Path Loss Models.

Mean path loss increases with distance proportional to about $d^{3}$ in hard-partitioned office buildings in both suburban and urban locations. As was found for other types of buildings, multiple floors between the transmitter and receiver lead to a large variability about the mean. In stores and sports arenas that have large open areas, path loss increases nearly as in free space. 
From Table 3.1-1, it can be seen that mean path loss exponents range between 1.4 and 4.3 with the value being highly dependent on the specific building. Even within the same building, highly varying surroundings such as the number of floors between transmitter and receiver cause large variability in path loss about the mean. In general, openplan buildings suffer less path loss for a given T-R separation than soft-partitioned buildings, and soft-partitioned buildings suffer less path loss than hard-partitioned buildings. This is likely due to the nature of the obstructions between transmitter and receiver in the different environments. In open-plan buildings, obstructions do not completely block the transmitter and receiver. In partitioned buildings, the partitions attenuate the signals, and hard partitions attenuate more than soft partitions.

\subsection{RMS Delay Spread Results}

The rms delay spread affects the maximum data rate that can be sent through a channel without equalization or diversity. Median rms delay spreads in open-plan buildings have been reported between 15 and $100 \mathrm{~ns}$. The size of the open-plan area affects the median delay spread since many of the multipath components are due to reflections from the exterior walls of the building. Maximum rms delay spreads were generally around 130 ns with 300 ns observed for a factory floor in [Rap89b], and $440 \mathrm{~ns}$ for a multi-floor office building in [Te191].

In soft-partitioned buildings, median rms delay spreads were around 75 ns with a maximum of $510 \mathrm{~ns}$ occurring when the transmitter and receiver were on separate floors [Te191]. Currently, there are limited data concerning delay spread in soft-partitioned environments.

For most of the measurements in hard-partitioned buildings, median rms delay spreads were on the order of $\mathbf{3 0}$ nanoseconds and maximum rms delay spreads were less than $70 \mathrm{~ns}$. The large partitions that separate offices from main corridors serve to attenuate the amplitudes of multipath components with large excess delays. In some cases, reflections from surrounding objects such as a hill outside the building [Dev86] and other highrise buildings [Sei92c] cause much higher delay spreads. Reference [Sei92c] shows that 
rms delay spreads as large as 1.47 microseconds can occur when the transmitter and receiver are on separate floors of an urban high-rise building.

In [Dev87a], two dissimilar office buildings were observed to have similar multipath power delay profiles and similar rms delay spread distributions. The maximum observed rms delay spread was 250 ns. The similarity in the delay spread distributions indicates that the building surroundings as well as the building itself should be considered when trying to approximate maximum delay spreads without measurements [Dev87a].

The measurements classified as residences in Table 3.1-1 were conducted for indoor to outdoor radio paths, whereas the transmitter and receiver were both inside the building for the other measurements. Multipath components were reported to have significant energy out to excess delays of around $1 \mu \mathrm{s}$. Maximum observed rms delay spreads were on the order of $100 \mathrm{~ns}$ when a direct path existed between the transmitter and receiver and were as large as $420 \mathrm{~ns}$ when no direct path was present [Dev86].

For measurements where the transmitter and receiver were on the same floor, rms delay spreads were generally larger in open-plan buildings than soft-partitioned environments. Similarly, rms delay spreads were larger in soft-partitioned environments than hard partitioned environments. As with path loss, it is likely that the nature of the obstructing objects contribute heavily to the delay spread. In open-plan buildings, multipath components can traverse a path from the transmitter to an exterior wall and then be scattered to the receiver. Such paths generally have larger time delays than multipath components that are scattered by objects in the interior of the building. In partitioned environments, the partitions serve to shadow far away scattering objects. When the transmitter and receiver are separated by multiple floors, multipath components that leave and enter the building through the windows and are scattered by surrounding buildings have been detected with excess delays of $7 \mu \mathrm{s}$ that lead to rms delay spreads of $1.47 \mu \mathrm{s}$ [Te191]. 


\subsection{The Effects of Carrier Frequency on Propagation Characteristics}

The effects of frequency on radio coverage in buildings were examined in [Mot88]. The mean path loss exponents were found to be nearly the same at both 900 $\mathrm{MHz}$ and $1700 \mathrm{MHz}$, but the attenuation caused per floor was $6 \mathrm{~dB}$ higher at $1700 \mathrm{MHz}$ than at $900 \mathrm{MHz}$. In addition, the path loss was $5.5 \mathrm{~dB}$ higher at $1700 \mathrm{MHz}$ due to the antenna aperture difference at the two frequencies. Thus, [Mot88] found the coverage to be reduced at $1700 \mathrm{MHz}$ as compared to $900 \mathrm{MHz}$.

Work in [Dev90a], [Haw90], [Mot90], [Dev91a], [Dev91b] showed virtually no statistical difference in path loss from $900 \mathrm{MHz}$ to $5.8 \mathrm{GHz}$. When multiple frequencies were studied [Dev90a], [Dev91a], [Dev91b], no significant statistical difference in time delay spread was found between $850 \mathrm{MHz}$ and $5.8 \mathrm{GHz}$. The path loss and shape of measured power delay profiles at $850 \mathrm{MHz}, 1.7 \mathrm{GHz}, 4.0 \mathrm{GHz}$, and $5.8 \mathrm{GHz}$ were reported to be similar. Virtually no difference in rms delay spread was found in four different buildings for $1.3 \mathrm{GHz}$ and $4.0 \mathrm{GHz}$ carrier frequencies [Haw91].

In [Bul89], two buildings were measured at $910 \mathrm{MHz}$ and $1.75 \mathrm{GHz}$. In one building, rms delay spreads were greater at $910 \mathrm{MHz}$, and in the other, delay spreads were greater at $1.75 \mathrm{GHz}$. The coherence bandwidths for the two frequencies were similar. Based on the current body of literature, there appears to be no significant effects of frequency on channel parameters such as path loss and delay spread for frequencies in the low microwave band $(1-6 \mathrm{GHz})$.

\subsection{Small-Scale Narrow Band Fading Statistics}

In [Bul89], it was reported that the dynamics of signal fading were slightly less random at $910 \mathrm{MHz}$ than at $1.7 \mathrm{GHz}$ and the average depths of fades were approximately $10 \mathrm{~dB}$ larger at $1.7 \mathrm{GHz}$. Although the severity of fading was always greater at $1.7 \mathrm{GHz}$, the severity of fading differed from building to building. Since each multipath component undergoes a $2 \pi$ phase shift for each wavelength traveled, it is expected that higher frequency signals will fade more rapidly due to the constructive and destructive interference of the phasor sum. In [Rap89a], the cumulative distribution functions of narrow band sig- 
nals as a receiver was moved along a one meter path were shown to be modeled by Rayleigh, Ricean, and Log-normal distributions about the median for different measurement runs. Cumulative distribution functions of received signal strength when both terminals were stationary were shown to be Ricean (K 10 dB) [Rap89a].

\subsection{SIRCIM}

Table 3.1-1 provides mean path loss exponents and median and maximum rms delay spreads measured in many different buildings. However, there is no information concerning the structure of the impulse response, or how parameters such as rms delay spread change as a mobile terminal is moved throughout a building over large-scale and over small-scale areas. Models that describe the amplitudes and time delays of multipath components in detail according to the impulse response channel model in Section 2.2 Multipath Channel Model are presented in [Rap91a] and were developed in [Sei89]. Based on the models in [Rap91a], a simulation package SIRCIM (Simulation of Indoor

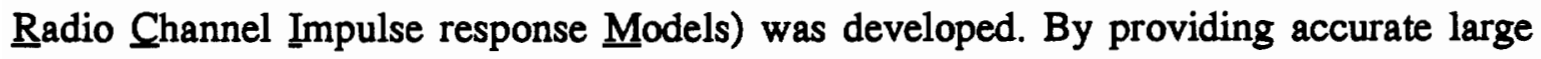
scale and small scale propagation fading data, SIRCIM can be used to study channel access, handoff, co-channel interference, equalization, diversity and modulation performance and bit error rates in frequency-flat and frequency-selective fading open-plan building environments.

SIRCIM is based on extensive propagation measurements at $1300 \mathrm{MHz}$ in [Rap89a], [Rap89b], and at $1300 \mathrm{MHz}$ and $4.0 \mathrm{GHz}$ in [Haw91], although published work in other frequency bands show agreement with the modes in SIRCIM (see Table 3.1-1 and Table 3.5-1). Both wide band and narrow band propagation characteristics from Table 3.1-1 were included in the propagation model. The wide band measurements used a pulsed RF transmitter similar to the one described in Section 3.2 Direct Pulse Measurements and Data Processing with a pulse duration of 7.8 ns [Rap89b]. Narrow band measurements were made with a modified commercial receiver [Rap89a]. Based on the measurements, statistical models for the number of distinct multipath signals, and the arrival times, and amplitudes of individual multipath components were developed. SIRCIM recreates the statistics of measured wide band impulse responses. By constructing the phases of individ- 
ual multipath components, SIRCIM also simulates narrow band frequency-flat fading channels since the instantaneous narrow band signal is the phasor sum of the individual multipath components in a particular impulse response.

The models which describe the impulse responses were developed for open-plan, soft-partitioned, and hard-partitioned buildings. The simulated values for path loss and rms delay spread are given in Table 3.5-1.

TABLE 3.5-1: Values of mean path loss exponent $\boldsymbol{n}$ and standard deviation $\sigma$ for SIRCIM [Rap90b].

\begin{tabular}{|c|c|c|c|c|}
\hline Topograpliy & $n$ & $\sigma(\mathrm{dB})$ & Velay spregr & 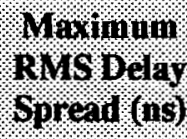 \\
\hline Open-Plan LOS & 1.9 & 6.5 & 87 & 173 \\
\hline Open-Plan OBS & 2.4 & 5.5 & 104 & 175 \\
\hline Hard-Partitioned LOS & 2.3 & 6.2 & 25 & 160 \\
\hline Hard-Partitioned OBS & 3.4 & 7.3 & 30 & 149 \\
\hline Soft-Partitioned LOS & 2.6 & 6.7 & 25 & 80 \\
\hline Soft-Partitioned OBS & 3.2 & 7.4 & 34 & 128 \\
\hline
\end{tabular}

The statistics of simulated and measured wide band impulse responses in openplan buildings were shown to be nearly identical in [Sei89], [Rap91c]. SIRCIM can also simulate narrow band signals over local areas directly without information about the wide band impulse responses at a particular location. The simulation method in [Smi75] is used to generate Rayleigh fading over a $1-\mathrm{m}$ track. For stationary terminals, a Ricean signal is simulated with a user-specified $\mathrm{K}$ ratio of specular-to-diffuse multipath power.

Computation of narrow band signal strengths as a function of small-scale mobile movements makes SIRCIM useful for studying channel access, handoff, and diversity. Bit error rates in narrow band frequency-flat fading channels for various modulation techniques may be investigated through simulation. Results are given in [Rap91c]. In [Tho92], the SIRCIM channel model was used to show that the structure of the power delay profile must be used to determine bit error rates. It was shown that two power delay profiles that had the same rms delay spread, but different time delay structures and phases give quite different bit error rates for the two channels. 
In mobile radio channels, received power is a function of T-R separation. Thus, SIRCIM may be used to compute coverage areas, outage probabilities, and co-channel interference constraints in future indoor microcellular environments on a statistical basis. The wide band impulse responses generated by SIRCIM recreate the statistics of individual multipath components as a mobile receiver moves. Thus, equalization or diversity techniques which attempt to adaptively cancel or track the multipath may be developed and tested with this channel simulator. With the recent interest in wide band multiple access techniques such as CDMA spread-spectrum communications, it makes sense to use a channel simulator such as SIRCIM which accurately simulates wide band path loss, rms delay spread, and multipath signal strength. SIRCIM is a commercially available software product that has been purchased by over fifty companies and universities conducting research in wireless communications. Independently developed multipath channel impulse response models similar to SIRCIM were presented in [Gan89].

Models like SIRCIM are valuable for evaluating system performance on a statistical basis. However, only limited information about the building is included. Site-specific path loss and delay spread models would allow more efficient system design and analysis.

\subsection{Site-Specific Path Loss Models}

Values of the mean path loss exponent and rms delay spread for many different building types were given in Table 3.1-1. However, the standard deviations of the path loss about the mean are as large as $16 \mathrm{~dB}$ in some cases. In addition, only the distance between the transmitter and receiver is incorporated into the model. No information about the specific locations of surrounding scattering objects is included. Many of the references report that the number of floors between transmitter and receiver highly influenced the observed path loss. This section presents some of the site-specific models for path loss caused by floors, walls, and soft-partitions. Two different models for path loss in multi-floored environments are presented and examples of how to use the models are given. A model for the attenuation caused by soft-partitions is given. Contour plots of the difference between measured and predicted path loss show that the model is more accurate than a statistical $\mathrm{d}^{\mathbf{n}}$ 
model that incorporates only T-R separation. Finally, values for the shadowing caused by common obstructions found in buildings are given.

In [Mot88], [Laf90], [Sei92a] the path loss caused by floors, hard (fixed) office walls, and soft (movable) office partitions were quantified to give more accurate propagation models that have lower spreads of path loss about the mean predicted value than simple $\mathrm{d}^{\mathrm{n}}$ models. Typical hard-partitioned office buildings were modeled in [Laf90] to give a prediction error standard deviation of $3 \mathrm{~dB}$.

Table 3.1-1 summarizes the mean path loss exponents and standard deviations about the mean for different environments. From Table 3.1-1, it can be seen that the parameters for path loss prediction for many buildings have large standard deviations. Large values are typical for data collected from different environments even within the same building. These parameters may be used in the model for a first-order prediction of mean signal strength when only T-R separation but no specific building information is known, but this model is clearly unsatisfactory for detailed site layout or capacity prediction.

\subsubsection{Multi-Floor Path Loss Exponent [Sei92a]}

In multi-floored environments, equation (3.6-1) can be used to describe the mean path loss as a function of distance. Equation (3.6-1) is identical to equation (2.3-2) and emphasizes that the mean path loss exponent is a function of the number of floors between transmitter and receiver. The values of $n$ (multi-floor) are given in Table 3.6-1 for use in equation (3.6-1).

$$
P L(d)[\mathrm{dB}]=P L\left(d_{0}\right)[\mathrm{dB}]+10 \times n(\text { multi-floor }) \times \log _{10}\left(\frac{d}{d_{0}}\right)
$$


TABLE 3.6-1: Multi-floor path loss exponents measured in [Sei92a]. Path loss values from both buildings were combined to provide average multi-floor path loss exponents.

\begin{tabular}{|c|c|c|}
\hline Number of Tloors & $101016100 \%)$ & $0(\mathrm{~dB})$ \\
\hline Same Floor & 2.76 & 12.9 \\
\hline One Floor & 4.19 & 5.1 \\
\hline Two Floors & 5.04 & 6.5 \\
\hline Three Floors & 5.22 & 6.7 \\
\hline
\end{tabular}

\subsubsection{Floor Attenuation Factor (FAF) Path Loss Model [Sei92a],[Sei92c]}

In the previous section, the path loss in multi-floored environments was predicted by a mean path loss exponent that was a function of the number of floors between transmitter and receiver. Alternatively, a constant Floor Attenuation Factor (dB), which is a function of the number of floors and building type, may be added to the mean path loss predicted by a path loss model which uses the same floor path loss exponent for the particular building type (equation (3.6-2)).

$P L(d)=$

$$
P L\left(d_{0}\right)[\mathrm{dB}]+10 \times n(\text { same floor }) \times \log _{10}\left(\frac{d}{d_{0}}\right)+F A F[\mathrm{~dB}]
$$

where $d$ is in meters and PL $\left(d_{0}\right)$ is due to free space. Same floor mean path loss exponents for the measurements in [Sei92a] are given in Table 3.6-2.

TABLE 3.6-2: Table of same-floor mean path loss exponents from [Sei92a].

\begin{tabular}{|c|c|c|}
\hline Builiting & 11 & $0(\mathrm{~dB})$ \\
\hline All Four Buildings & 2.76 & 12.9 \\
\hline Grocery Store & 1.81 & 5.2 \\
\hline Retail Store & 2.18 & 8.7 \\
\hline Office Building 1 & 3.27 & 11.2 \\
\hline Office Building 2 & 3.25 & 5.2 \\
\hline
\end{tabular}


TABLE 3.6-3: Table of Floor Attenuation Factor measured in five buildings in [Sei92a] and [Sei92c]. Note that the method used to determine the FAF was slightly different in [Sei92c] than in [Sei92a].

\begin{tabular}{|c|c|c|c|}
\hline Number of Floons & Reference & 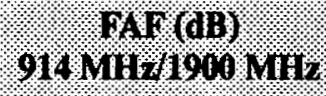 & 914 . \\
\hline Office Building : 1 & [Sei92a] & & \\
\hline One Floor & & $12.9 /-$ & $7.0 /-$ \\
\hline Two Floors & & $18.7 /-$ & $2.8 \%$ \\
\hline Three Floors & & $24.4 /-$ & $1.7 /-$ \\
\hline Four Floors & & $27.0 \%$ & $1.5 \%$ \\
\hline Office Building : 2 & [Sei92a] & & \\
\hline One Floor & & $16.2 /-$ & $2.9 \%$ \\
\hline Two Floors & & $27.5 /-$ & $5.4 /-$ \\
\hline Three Floors & & $31.6 /-$ & $7.2 \%$ \\
\hline Urban High Rise & [Sei92c] & & \\
\hline One Floor & & $13.2 / 26.2$ & $9.2 / 10.5$ \\
\hline Two Floors & & $18.1 / 33.4$ & $8.0 / 9.9$ \\
\hline Three Floors & & $24.0 / 35.2$ & $5.6 / 5.9$ \\
\hline Four Floors & & $27.0 / 38.4$ & $6.8 / 3.4$ \\
\hline Five Floors & & $27.1 / 46.4$ & $6.3 / 3.9$ \\
\hline $\begin{array}{l}\text { Suburban Office } \\
\text { Building - Open }\end{array}$ & [Sei92c] & & \\
\hline One Floor & & $33.6 / 31.3$ & $3.2 / 4.6$ \\
\hline Two Floors & & $44.0 / 38.5$ & $4.8 / 4.0$ \\
\hline $\begin{array}{l}\text { Suburban Office } \\
\text { Building - Soft } \\
\text { Partitioned }\end{array}$ & [Sei92c] & & \\
\hline One Floor & & $29.1 / 35.4$ & $5.8 / 6.4$ \\
\hline Two Floors & & $36.6 / 35.6$ & $6.0 / 5.9$ \\
\hline Three Floors & & $39.6 / 35.2$ & $6.0 / 3.9$ \\
\hline
\end{tabular}

Table 3.6-3 gives the Floor Attenuation Factors and the standard deviations (in dB) of the difference between the measured and predicted path loss in [Sei92a] and [Sei92c]. In [Sei92a], the Floor Attenuation Factors were computed as the difference between the measured path loss and the mean path loss predicted by a $\mathrm{d}^{\mathrm{n}}$ model where $n$ was the same floor mean path loss exponent in Table 3.6-2 for the particular building structure and $d$ was the shortest distance between the transmitter and receiver measured in three dimensions. 
In [Sei92c], the Floor Attenuation Factors were computed as the difference between measured and free space path loss over the same distance.

The average Floor Attenuation Factors for an identical number of floors between the transmitter and receiver for the two buildings differed by 3-8 dB in [Sei92a]. All floors in the two office buildings were made of reinforced concrete. Office building 1 was built within the past ten years, and office building 2 was 20 to 30 years old. Both buildings were longer and wider than they were high. It is interesting to note that in [Sei92a], [Sei92c], the average FAF was not a linear function of the number of floors between the transmitter and receiver as was found in [Mot88], [Mot90]. It is possible different floors cause different amounts of path loss, and other factors such as multipath reflections from surrounding buildings may affect the path loss [Sei92c]. In [Arn89], cross-floor isolation was $26 \mathrm{~dB}$ for metal floors between the transmitter and receiver, and the isolation was only $10 \mathrm{~dB}$ when the floors were not metal. More measurements are called for in many multi-floored buildings to quantify the Floor Attenuation Factors as a function of frequency and building materials, with the goal that eventually, the loss between different floors in buildings may be predicted without measurements.

\subsubsection{Application of Path Loss Models in Multi-Floored Environments [Sei92a]}

As an example of how to use the two different models to predict the mean path loss through three floors of office building 1 , assume the mean path loss exponent for same floor measurements in a building is $n=3.27$, the mean path loss exponent for three-floor measurements is $n=5.22$, and the average Floor Attenuation Factor is FAF=24.4 dB for three floors between transmitter and receiver. These parameters were found from Table 3.6-1 through Table 3.6-3 for office building 1 in [Sei92a]. Then, at a T-R separation of $\mathrm{d}=30.0 \mathrm{~m}$, the predicted mean path loss at $915 \mathrm{MHz}$ using (3.6-1) is

$$
\begin{array}{r}
P L(30 \mathrm{~m})[\mathrm{dB}]=P L(1 \mathrm{~m})[\mathrm{dB}]+10 \times 5.22 \times \log _{10}(30) \\
=108.8 \mathrm{~dB}
\end{array}
$$


or, using (3.6-2),

$P L(30 \mathrm{~m})=$

$=P L(1 \mathrm{~m})[\mathrm{dB}]+10 \times 3.27 \times \log _{10}(30)+24.4=104.4 \mathrm{~dB}$

where $\mathrm{PL}(1 \mathrm{~m})=31.7 \mathrm{~dB}$.

\subsubsection{Path Loss Contour Plots [Sei92a]}

One way to visualize the effects of a building environment on path loss is to determine a contour plot of locations of equal path loss. In [Sei92a], building blueprints and both measured and predicted path loss data were imported to a computer-aided design program (AutoCAD). The data were used to form contour plots of locations of equal path loss for a given transmitter location. In each figure, the transmitter location is indicated by an arrow pointing to an ' $X$ ' at the transmitter location. Curved solid lines indicate locations of equal path loss from the transmitter in $10 \mathrm{~dB}$ steps. The amount of path loss is indicated at the end of the lines on the perimeter of the blueprints.

The contour plot of locations with equal measured path loss for the West wing on the fifth floor of office building 1 in [Sei92a] is given in Figure 3.6-1. The transmitter was located in the upper right hand corner of the figure as indicated. The thin lines on the drawing indicate 1.5 meter high soft partition cubicle dividers. In the center of the wing are conference rooms with concrete block walls which span from the floor to the ceiling. In Figure 3.6-1, notice that when the thick conference room walls are between the receiver and the transmitter, the signal is attenuated much more rapidly than at other locations. Along the diagonal hallway along the edge of the building, the radio coverage is quite good, and obeys better than free space propagation. Propagation better than in free space was also observed in [Rap89a], [Rap89b], [Laf90], [Te191] (and others) for open hallways that can guide signal energy.

The contour plot for the fourth floor west wing of office building 1 in [Sei92a] is shown in Figure 3.6-2. The transmitter was located inside an office cubicle on the left side 


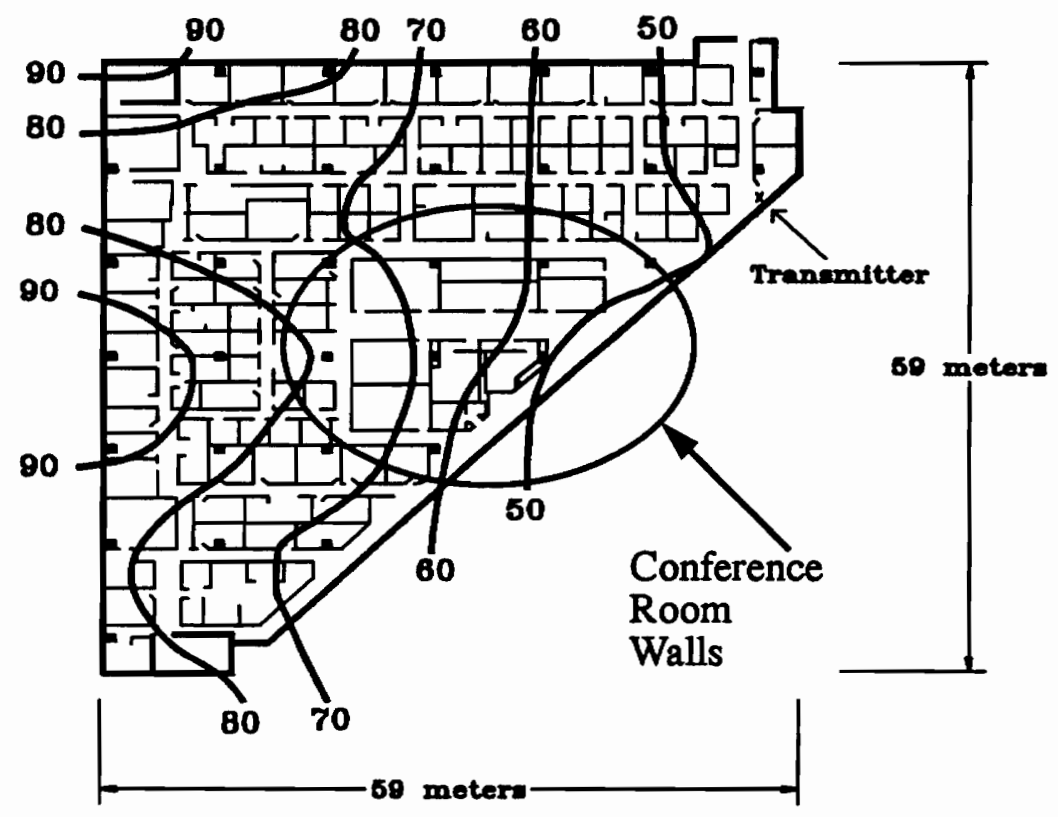

FIGURE 3.6-1. Contour plot of measured path loss for the fifth floor of offic building 1 in [Sei92a].

of the figure. The thin lines which surround rectangular areas represent the perimeters of soft-partitioned office cubicles, not necessarily the locations of the partitions. Both the transmitter and the receiver antenna were $1.0 \mathrm{~m}$ above the floor. The path loss seems to be consistent with T-R separation and is not greatly affected by the direction of propagation.

\subsubsection{Soft Partition and Concrete Wall Attenuation Factor Model [Sei92a]}

The previous models include the effects of T-R separation, building type, and the number of floors between the transmitter and receiver, and are a first step for including site information to improve propagation prediction. In [Laf90] hallways and rooms adjacent to a main corridor, and in [Mot90], floors and plasterboard partitioned walls were considered in the development of site-specific models. In [Dev90b], a model was proposed that models path loss as $d^{n}$ plus $\alpha \mathrm{dB} /$ meter. Although this models the measured data, no physical explanation was given to determine $\alpha$ for different environments.

There are often obstructions between the transmitter and receiver even when the terminals are on the same floor. Consider the path loss effects of soft partitions and con- 


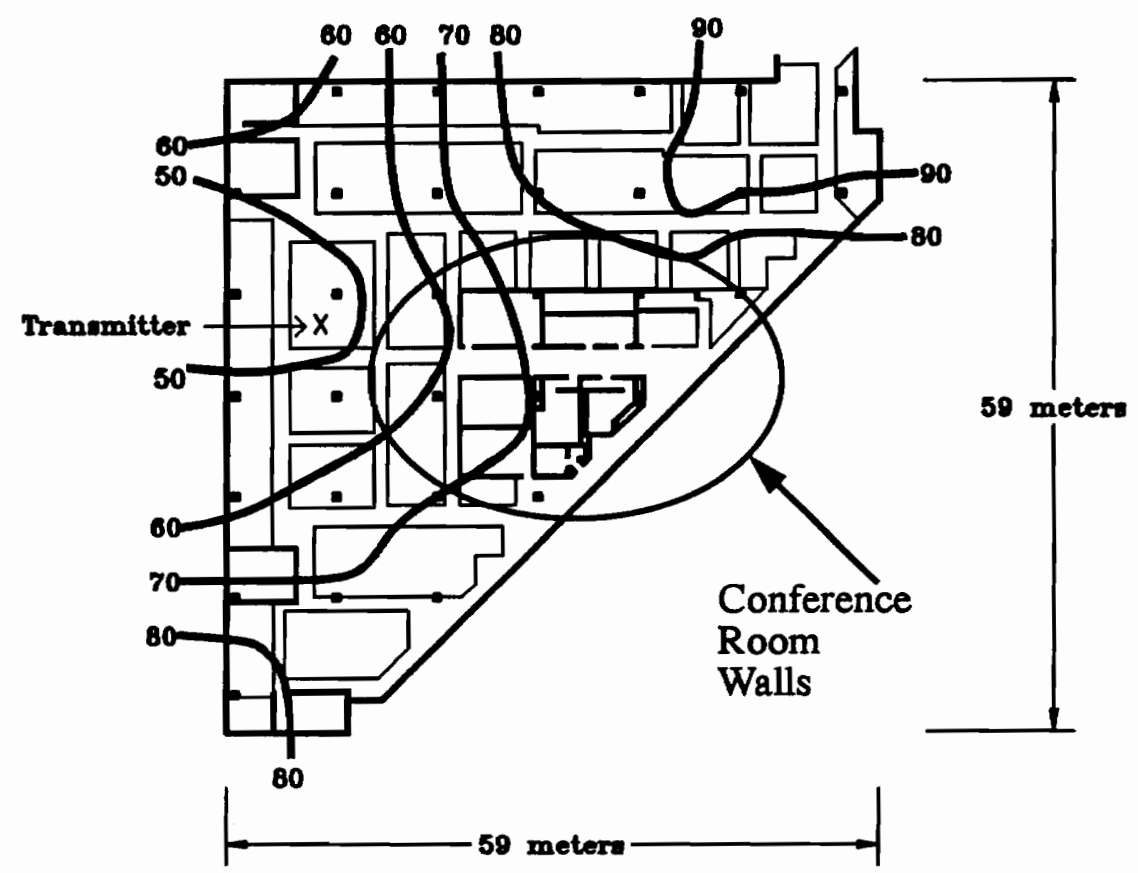

FIGURE 3.6-2. Contour plot of measured path loss for the fourth floor of office building 1 in [Sei92a]

crete walls between the transmitter and receiver for same floor measurements in soft-partitioned environments in [Sei92a]. For a physical model that will apply to all softpartitioned environments, it was assumed path loss increases with distance as in free space $(n=2)$, so long as there are no obstructions between the transmitter and receiver. Then, Attenuation Factors (AF) for each soft partition and concrete wall that lie directly between the transmitter and receiver were included. For simplicity, any kind of concrete support column that wholly or partially blocks the direct path between the transmitter and receiver was labeled a concrete wall. Table 3.6-4 summarizes the variables used to describe the soft-partition and concrete wall attenuation factor model. 
TABLE 3.6-4: Summary of the variables used to describe the soft partition and concrete wall attenuation factor model.

\begin{tabular}{|c|c|}
\hline Wuriable & Decription /uingly \\
\hline d & T-R separation [m] \\
\hline PL & Path loss [dB] \\
\hline$\lambda$ & Carrier wavelength [m] \\
\hline $\mathbf{p}$ & Number of soft partitions between the transmitter and receiver \\
\hline $\mathbf{q}$ & Number of concrete walls (or posts) between the transmitter and receiver \\
\hline AF(soft partition) & Attenuation factor for one soft partition [dB] \\
\hline AF(concrete wall) & Attenuation factor for one concrete wall (or post) [dB] \\
\hline
\end{tabular}

Let $p$ be the number of soft partitions and $q$ be the number of concrete walls between the transmitter and receiver. The mean path loss predicted by the Attenuation Factor path loss model is then given by

$P L(d)[\mathrm{dB}]=20 \times \log _{10}\left(\frac{4 \pi d}{\lambda}\right)+p \times A F($ soft partition $)[\mathrm{dB}]+$

$$
q \times A F \text { (concrete wall) [dB] }
$$

Notice that no reference distance is used since free space propagation is assumed for all distances. This model considers only objects along the straight path between the transmitter and the receiver. No multipath effects are included in the model.

For each of the discrete path loss measurements in the soft partitioned environments in [Sei92a], the difference between the measured path loss and the path loss that would occur due to free space propagation for a transmitter and receiver at the same separation distance were computed. The number of soft partitions and the number of concrete walls (or concrete building support columns) between the transmitter and receiver were recorded. Linear regression was used to find the best fit, in a minimum mean square error sense, for the Attenuation Factors (AF) of each soft partition and each concrete wall between the transmitter and receiver, where all soft partitions were assumed to induce identical path loss, and all concrete walls were assumed to induce identical path loss. The 
standard deviation in $\mathrm{dB}$ of the difference between the measured path loss and the path loss predicted by equation (3.6-5) was computed in [Sei92a].

When all path loss measurements in soft-partitioned environments in [Sei92a] were considered, the $\mathrm{AF}$ was $1.39 \mathrm{~dB}$ for each soft partition and $2.38 \mathrm{~dB}$ for each concrete wall between the transmitter and receiver. Although these values may seem low for the attenuation through a partition and a concrete wall, these values were determined to give the best-fit between measured and propagation data based on a model that considers only objects on a direct line between the transmitter and receiver. Other propagation paths may have contributed to the actual received power which would reduce the apparent attenuation factors. Figure 3.6-3 shows a scatter plot of the actual measured path loss vs. the path loss predicted by equation (3.6-5) with $\mathrm{AF}$ (soft partition) $=1.39 \mathrm{~dB}$ and $\mathrm{AF}$ (concrete wall $)=2.38 \mathrm{~dB}$ for the three soft-partitioned environments. The diagonal straight line in Figure 3.6-3 shows where measured and predicted path loss are identical. The standard deviation of the difference between measured and predicted path loss is $4.1 \mathrm{~dB}$. The softpartition and concrete wall Attenuation Factor Model in equation (3.6-5) explains the deviation of the mean path loss exponent from free space $(n=2)$ based on a physical model that assumes free space propagation with distance and attributes additional path loss to identifiable physical obstructions between the transmitter and receiver. Attenuation Factors for common objects found in buildings are given later in this section on site-specific models.

\subsubsection{Predicted Path Loss for Soft Partitioned Environments [Sei92a]}

The Soft Partition and Concrete Wall Attenuation Factor Model in equation (3.6-5) was used to predict path loss in soft partitioned environments in [Sei92a] with $\mathrm{AF}$ (soft partition) $=1.39 \mathrm{~dB}$ and $\mathrm{AF}$ (concrete wall) $=2.38 \mathrm{~dB}$. The differences between measured and predicted path loss were used to generate error contours. The absolute value of the error has been plotted to show regions where the model in equation (3.6-5) accurately predicts the path loss and regions where the model is less accurate. The path loss prediction error is proportional to the darkness of the shaded region. It is important to note that the error can be positive or negative within a single shaded region. This is done to indicate 


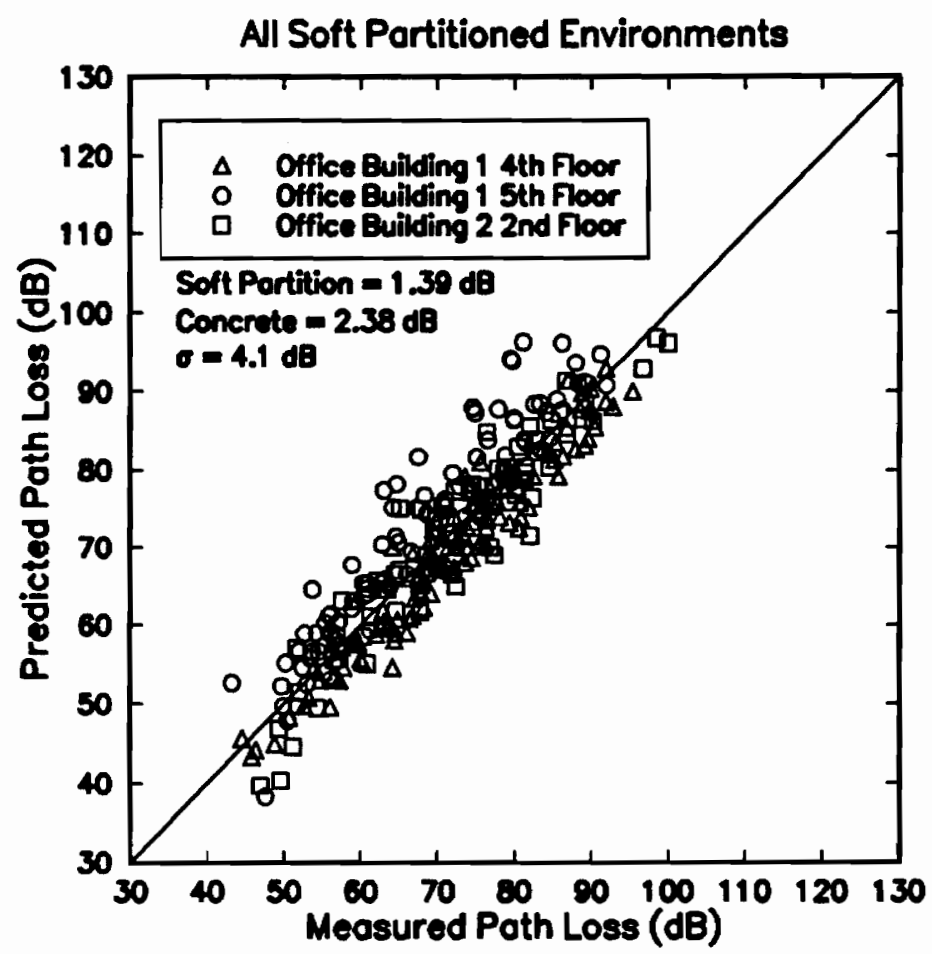

FIGURE 3.6-3. Scatter plot of measured and predicted path loss in softpartitioned environments as measured and predicted in [Sei92a]. The results were optimized over the combination of measurement locations in all three buildings.

regions where the models have difficulty predicting the path loss. Thus, the use of a correction factor for the shaded regions is not appropriate.

The error contours for the West wing of the fifth floor of office building 1 in [Sei92a] and the contours of measured path loss for the same transmitter location and building wing are given in Figure 3.6-4. The prediction error is less than $3 \mathrm{~dB}$ for about half of the area in this location. There are several places where the prediction error is greater than $9 \mathrm{~dB}$. This may be partly explained in that the Attenuation Factors for the fifth floor in the West wing of office building 1 were the lowest measured and differ by about $0.4 \mathrm{~dB}$ from the Attenuation Factors used to predict path loss. Thus, on average, $0.4 \mathrm{~dB}$ more path loss is predicted than was actually observed for each partition and concrete wall in this wing. When many partitions and concrete walls are between the transmitter and receiver, the bias can be 4-5 dB. 


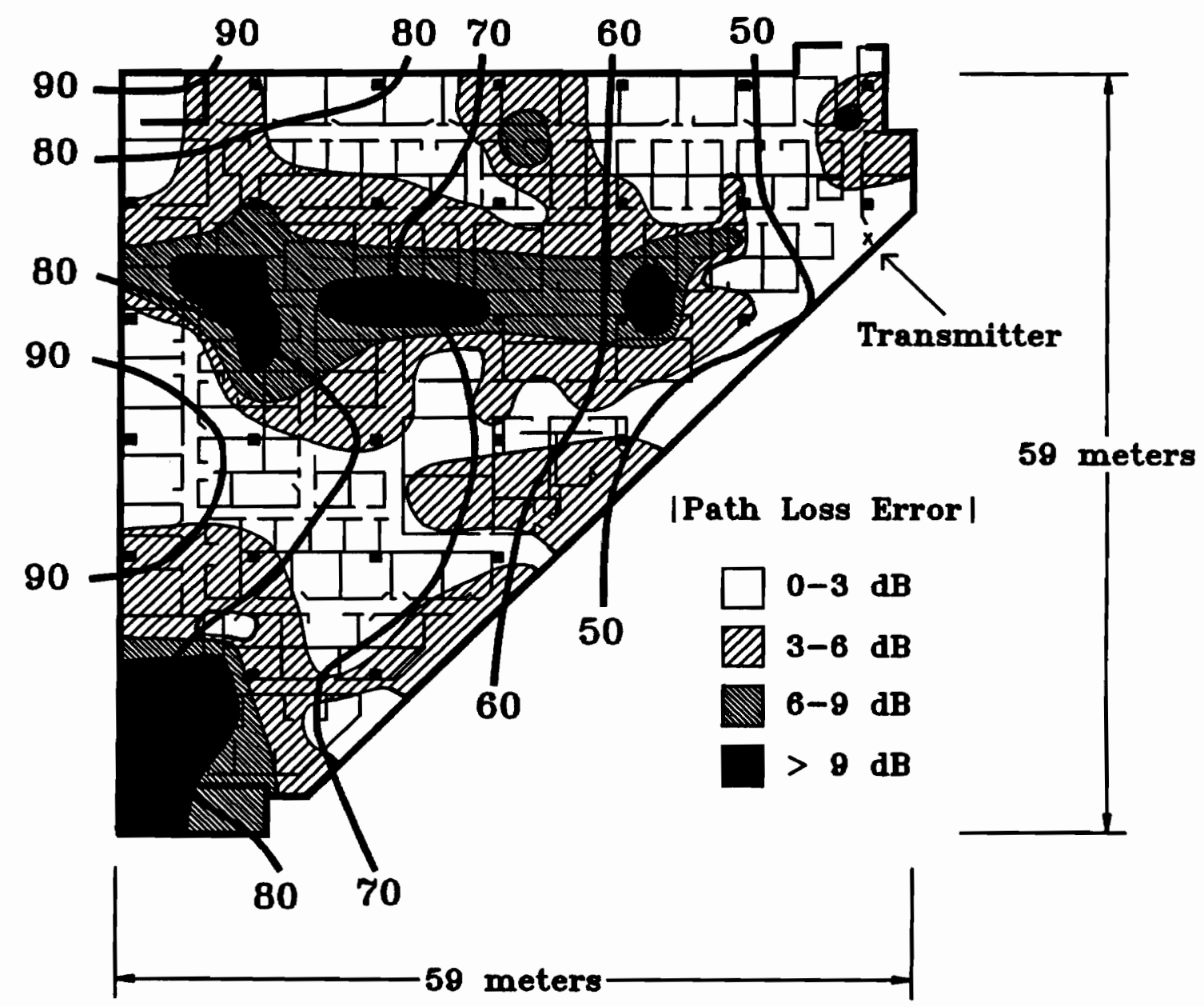

FIGURE 3.6-4. Error contour plot of predicted path loss for the soft partitioned West wing of the 5th floor of office building 1 in [Sei92a].

Figure 3.6-5 shows the measured and error contours for the fourth floor West wing of office building lin [Sei92a]. The prediction error is less than $3 \mathrm{~dB}$ for over half of the locations and less than $6 \mathrm{~dB}$ for nearly all of this wing. The error contours for this portion of the building show that equation (3.6-5) can be used to accurately predict path loss based on site-specific information.

\subsubsection{Shadowing Caused by Typical Objects Found in Buildings}

Although not an electromagnetically rigorous solution, the shadowing caused by typical objects found in indoor radio channels can be estimated from propagation measurements. Table 3.6-6 and Table 3.6-5 give typical shadowing caused by these objects. 


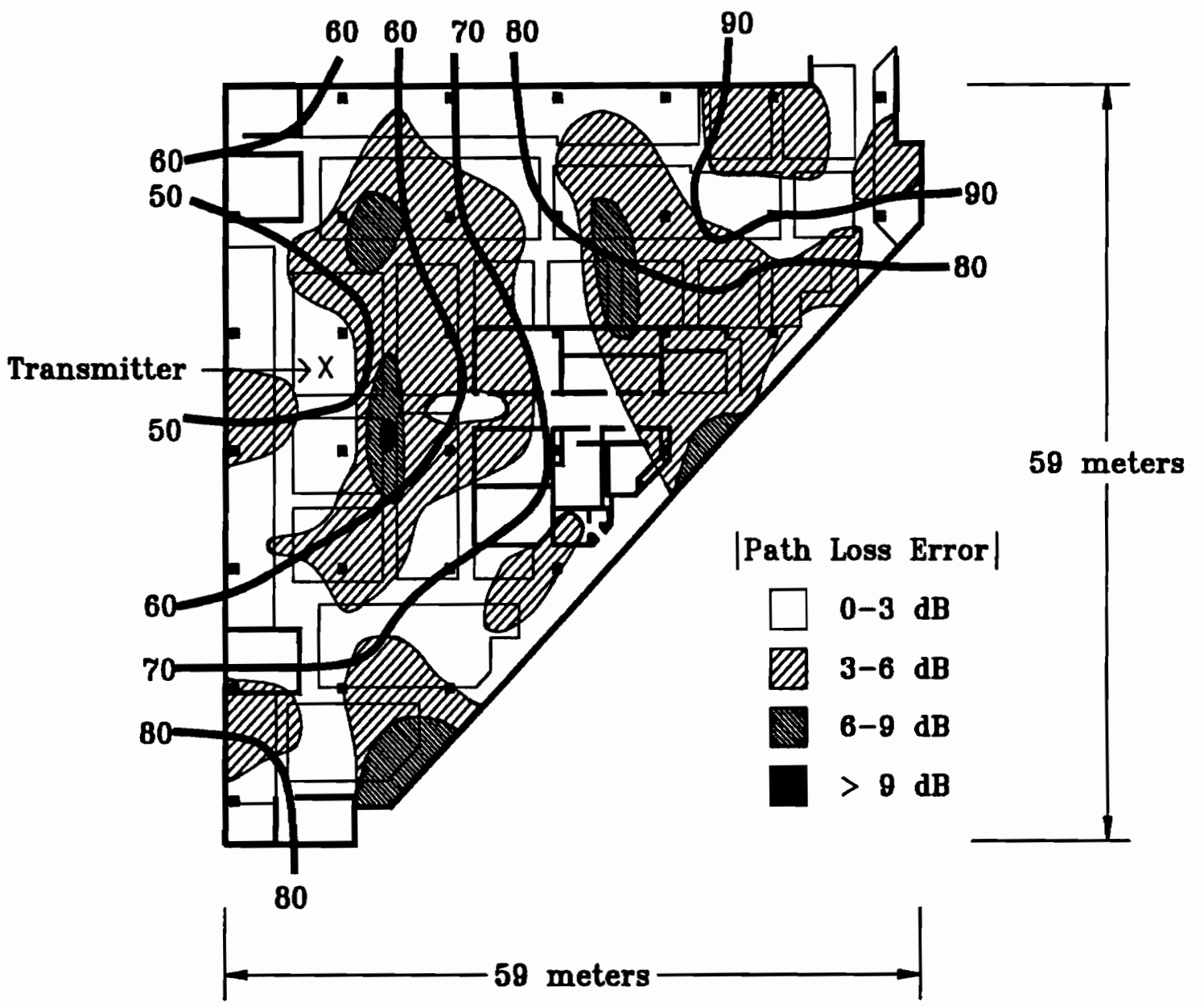

FIGURE 3.6-5. Error contour plot for the soft partitioned West wing on the 4th floor of office building 1 in [Sei92a].

These values provide rule of thumb estimates to the shadowing caused by common objects found in buildings. In [Rap89a], the shadowing was defined as the difference between the observed path loss behind the obstruction and the mean path loss predicted by the $d^{n}$ model (equation (2.3-2)) for the given T-R separation and building environment. In [Haw91], the shadowing was computed as the difference in the slow fading component of the signal as the mobile receiver moved from a shadowed to an unshadowed region. 
TABLE 3.6-5: Shadowing effects of common factory equipment measured at 1300 MHz in [Rap89a].

\begin{tabular}{|c|c|}
\hline Iten: & $1005(18)$ \\
\hline $2.5 \mathrm{~m}$ storage rack with small metal parts (loosely packed) & 4-6 \\
\hline $4 \mathrm{~m}$ metal box storage & 10-12 \\
\hline $5 \mathrm{~m}$ storage rack with paper products (loosely packed) & 24 \\
\hline $5 \mathrm{~m}$ storage rack with paper products (tightly packed) & 6 \\
\hline $5 \mathrm{~m}$ storage rack with large metal parts (tightly packed) & 20 \\
\hline Typical N/C machine & $8-10$ \\
\hline Semi-automated assembly line & $5-7$ \\
\hline $0.6 \mathrm{~m}$ square reinforced concrete pillar & $12-14$ \\
\hline Stainless steel piping for cook-cool process & 15 \\
\hline
\end{tabular}


TABLE 3.6-6: Shadowing effects of typical factory obstructions measured at 1300 MHz in [Haw91].

\begin{tabular}{|c|c|}
\hline theng & $100.8(\mathrm{~dB})$ \\
\hline Light textile inventory & $3-5$ \\
\hline Chain link fenced in area $20 \mathrm{ft}$. high which contains tools, inventory, and people & $5-12$ \\
\hline Metal blanker, 12 square feet & 4-7 \\
\hline Metallic hoppers which hold scrap metal for recycling. 10 square feet & $3-6$ \\
\hline Small metal pole, 6 inch diameter & 3 \\
\hline Metal pulley system used to hoist metal inventory, 4 square feet & 6 \\
\hline Light machinery, $<10$ square feet & $1-4$ \\
\hline General machinery, $10-20$ square feet & $5-10$ \\
\hline Heavy machinery, $>20$ square feet & $10-12$ \\
\hline Metal catwalk/stairs & 5 \\
\hline Light textile inventory & $3-5$ \\
\hline Heavy textile inventory & $8-11$ \\
\hline Area where workers inspect metal finished products for defects & $3-12$ \\
\hline Metallic inventory & 4-7 \\
\hline Large I-beam, 16-20 inches & $8-10$ \\
\hline Metallic inventory racks, 8 square feet & 4-9 \\
\hline Empty cardboard inventory boxes & $3-6$ \\
\hline Concrete block wall & $13-20$ \\
\hline Ceiling duct & $1-8$ \\
\hline
\end{tabular}

TABLE 3.6-7: Shadowing effects of typical office building obstructions measured at $1300 \mathrm{MHz}$ in [Haw91].

\begin{tabular}{|c|c|}
\hline $1+1+n$ & $10.5(\mathrm{~dB})$ \\
\hline Concrete block wall & 13 \\
\hline Loss from one floor & $20-30$ \\
\hline Loss from one floor and one wall & $40-50$ \\
\hline Fade observed when transmitter turned a right angle corner in a corridor & $10-15$ \\
\hline
\end{tabular}




\subsection{Summary}

This chapter has presented statistical results for path loss and delay spread in many different building types as compiled from the literature. Models for path loss as a function of distance and median and maximum rms delay spreads were presented for different buildings. Buildings with large open spaces typically have higher rms delay spreads than partitioned buildings unless signals that are scattered from surrounding buildings can be detected. Carrier frequency was shown to have little effect on the statistics of propagation parameters such as path loss and rms delay spread for carrier frequencies between 800 $\mathrm{MHz}$ and $5.8 \mathrm{GHz}$. Generic statistical models have progressed towards site-specific models of measured propagation data. The effects of multiple floors, concrete walls, partitions, and other shadowing objects have been quantified. In order to efficiently design future wireless systems, true propagation prediction that can accurately estimate signal levels and delay spread in different environments without measurements is required. The next chapter presents theoretical electromagnetic models for propagation, scattering, and diffraction. The merging of these models with site-specific building information is described to provide propagation prediction inside buildings which is more accurate than current statistical models. 


\section{Geometrical Optics, Scattering, and Diffraction}

\subsection{Overview}

Chapter 3 presented some statistical and heuristically developed site-specific models for propagation based on measurements in many different buildings. This chapter presents propagation models based on electromagnetic theory that can be used to relate the propagation to the specific environment. Propagation models based on theoretical models can be used to predict the propagation based on the surroundings of the transmitter and receiver without requiring measurements. The chapter begins with a general description of electromagnetic scattering by arbitrarily shaped bodies. Next, a geometrical optics model for high frequency wave propagation is described. This description includes a discussion of plane wave reflection and transmission at a dielectric interface. Several different scattering and diffraction models are described, and the usefulness of each to a site-specific propagation model is investigated. First, the geometrical theory of diffraction is presented as a natural extension of a geometrical optics propagation model. A physical optics scattering model and a heuristic scattering model for flat surfaces are discussed. These different scattering and diffraction models are used to investigate the scattering from a perfectly conducting smooth rectangular flat plate. Numerical results are presented to determine the region of validity for each model.

\subsection{Electromagnetic Scattering by Arbitrarily Shaped Bodies}

\subsubsection{Equivalence}

Consider a time-harmonic electromagnetic wave in a source-free region in the presence of an arbitrarily shaped scatterer as shown in Figure 4.1-1. The incident fields $\vec{E}_{i}$ and $\vec{H}_{i}$ are known. To solve for the total fields outside the scattering body requires solving Maxwell's equations for the fields both inside and outside the body. The appropriate boundary conditions on the surface of the body must also be satisfied. Instead of solving the complete Maxwell's equations, the problem may be simplified through application of the equivalence principle. The equivalence principle means that the original problem may 

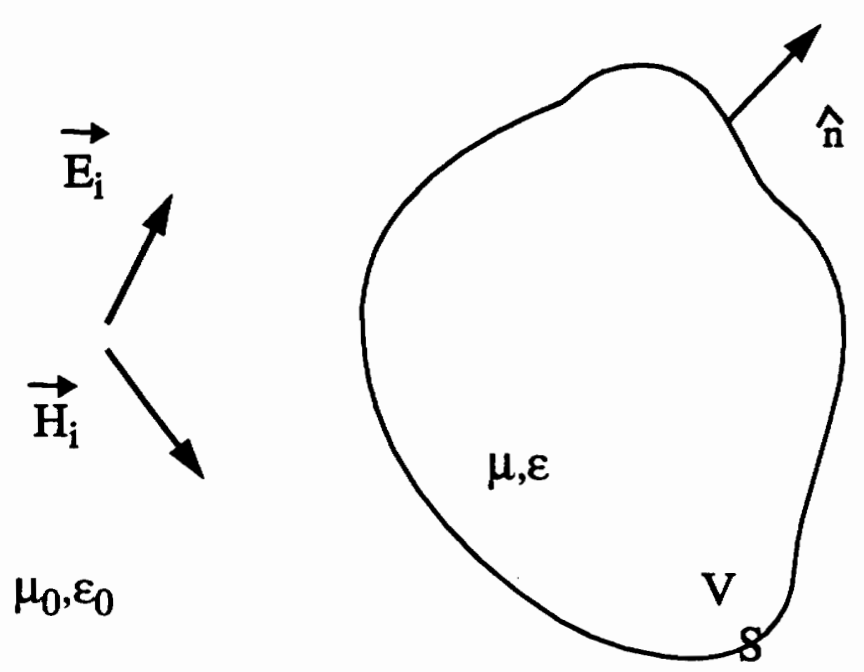

FIGURE 4.1-1. An electromagnetic wave in the presence of an arbitrarily shaped scatterer.

be replaced with an equivalent problem in order to solve for the fields in a particular region of interest [Har61]. The problems are only equivalent in the region of interest. The solution of the equivalent problem outside the region of interest is not valid. The total fields $\vec{E}_{T}$ and $\vec{H}_{T}$ in the region of interest are the sum of the incident fields $\vec{E}_{i}$ and $\vec{H}_{i}$ and the scattered fields $\vec{E}_{S}$ and $\vec{H}_{S}$. The scattered fields are defined as the difference between the fields in the presence of the body and the fields in the absence of the body (incident fields). To solve for the scattered fields outside the dielectric body, replace the problem in Figure 4.1-1 with the equivalent problem shown in Figure 4.1-2. The dielectric body is transformed to a body with the properties of the exterior region (free space). Equivalent surface currents are included so that the fields radiated by the surface currents are equivalent to the true scattered fields in the exterior region of interest. The surface current sources $\vec{J}_{s}$ and $\vec{M}$, radiating in free space produce the same total fields exterior to the body $\mathrm{V}$ and are therefore equivalent to the original problem in the exterior region.

The scattered fields can be determined from the surface currents that radiate in an unbounded homogeneous medium in the equivalent problem. The values of the surface currents are determined so that the total fields inside the body are zero. Recall that these fields are not the actual fields inside the body, since the solution is not valid in the transformed region. The surface currents are determined from $\vec{J}_{s}=\hat{n} \times \vec{H}_{i}$ and $\vec{M}_{s}=-\hat{n} \times \vec{E}_{i}$ 


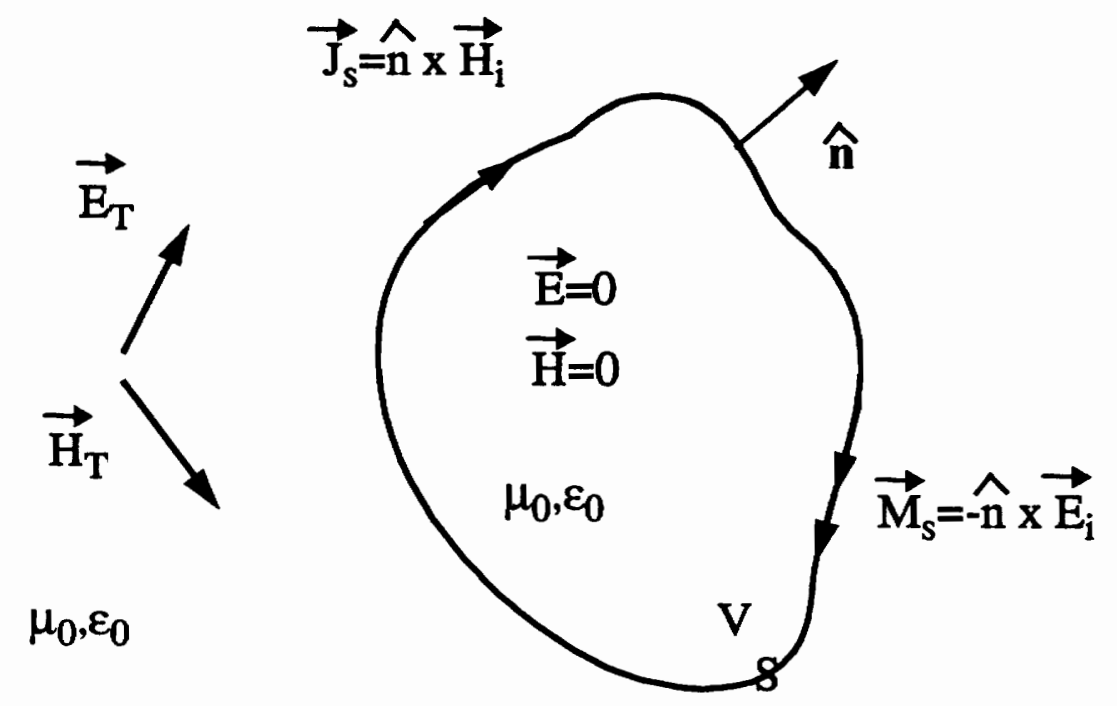

FIGURE 4.1-2. Equivalent problem in the exterior region for an electromagnetic wave in the presence of an arbitrarily shaped scatterer.

where $\hat{n}$ is the outward pointing surface normal. The scattering problem has been transformed into a radiation problem. Radiation and scattering are variations of the same process. Typically, the field scattered by a dielectric body is a spatial Fourier transform that depends on the size of the body, the field variation in the body, and the dielectric variation in the body. As an example, the pattern radiated by an antenna is expressed by a Fourier transform that depends on the size of the antenna and the current distribution on the antenna or the field distribution in the antenna aperture. The solution for the total fields in the presence of the scattering body solve Maxwell's equations everywhere and satisfy the boundary conditions on the surface of the scattering object. In general, this is a difficult problem to solve exactly.

\subsubsection{Characterization of Scattering Regimes}

When the surfaces of the scattering object coincide with the coordinate axes for a geometry in which the vector wave equation is separable, an analytic solution for the total field may be possible. The vector wave equation

$$
\nabla^{2} \vec{E}\left(x_{1}, x_{2}, x_{3}\right)-\mu \varepsilon \frac{\partial^{2}}{\partial t^{2}} \vec{E}\left(x_{1}, x_{2}, x_{3}\right)=0
$$


is separable if each of the vector components of the solution $\vec{E}\left(x_{1}, x_{2}, x_{3}\right)$ can be expressed in the form of $A\left(x_{1}\right) B\left(x_{2}\right) C\left(x_{3}\right)$. The variables $\mu$ and $\varepsilon$ are the permeability and permittivity of the medium where the fields exist, respectively. The three coordinate variables are represented by the variables $x_{1}, x_{2}$, and $x_{3}$. The vector wave equation is only separable in certain coordinate geometries such as rectangular coordinates, cylindrical, and spherical coordinates.

Certain approximations to the scattering solution can be made when the frequency is high or when the frequency is low. Consider a transmitter and two receivers in the presence of a scattering body. Figure 4.1-3 shows a transmitter, two receivers, and a scattering object. A direct path exists between the transmitter and receiver 1, but the scatterer blocks

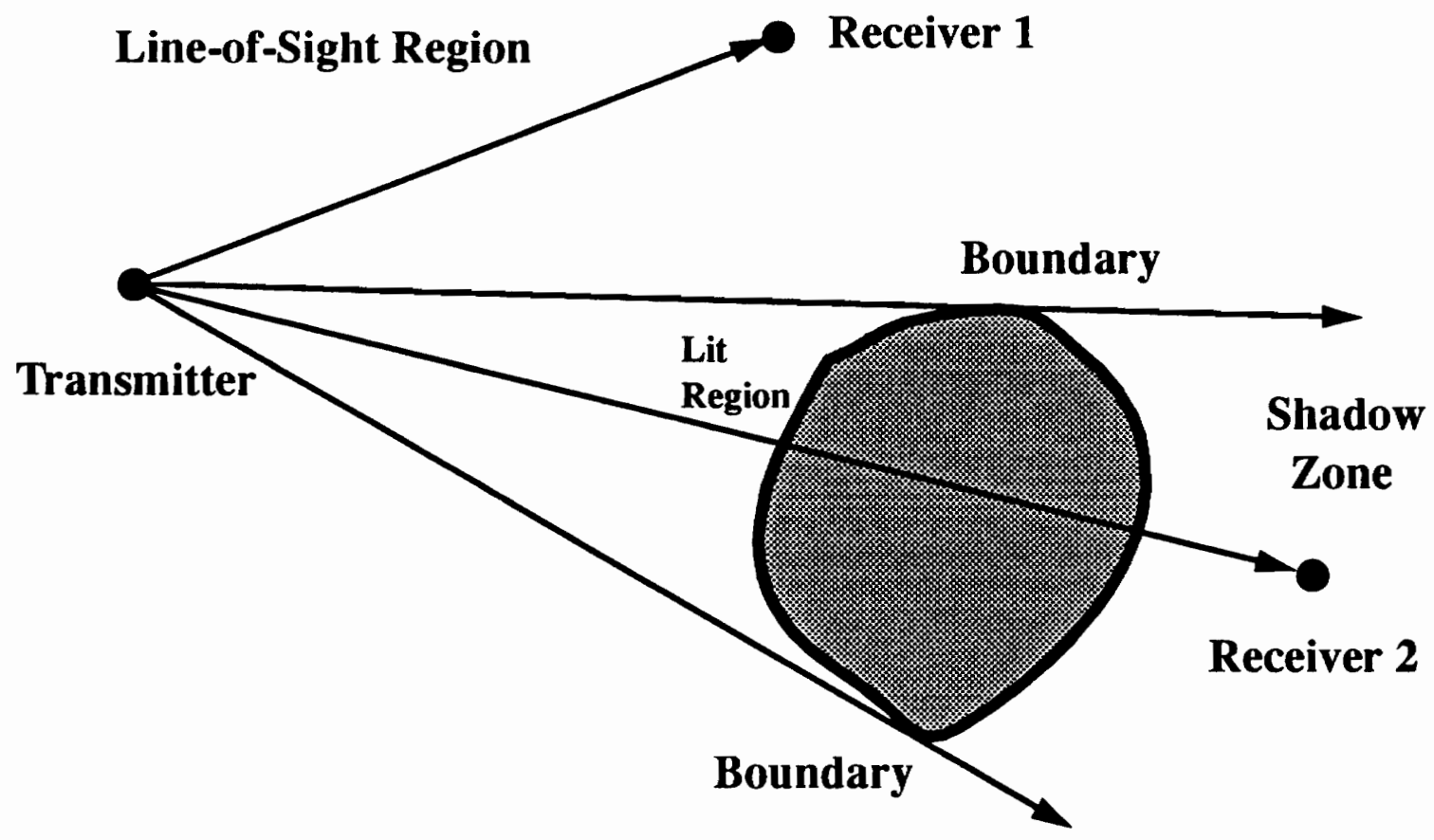

FIGURE 4.1-3. Shadow zone for a scattering object.

the direct path between the transmitter and receiver 2. Arrows are drawn to indicate the boundaries of the region where the scatterer blocks the direct path between the transmitter and receiver. This region where the direct path is blocked is called the shadow zone. All 
observation points not in the shadow zone are in the line-of-sight region. The locations on the surface of the body that are line-of-sight to the transmitter make up the lit region. The terms shadow zone and lit region follow from optical theory. Optical theory is the high frequency limit of electromagnetics since visible light is electromagnetic with very short wavelengths $(\lambda \sim 0.6 \mu \mathrm{m})$. Hence, the term shadow zone is used since this region is in the shadow of the object. High frequency implies that the wavelength is small. However, small is a relative measure of size, and the frequency may only be considered high with respect to some reference. In scattering problems, the wavelength must be much smaller than the size of the scattering body for high frequency limits to apply. This limit is useful for approximating the scattering that results from large objects such as building walls at microwave frequencies.

The other extreme is the low frequency limit. The low frequency limit applies when the wavelength is much larger than the scattering body. This limit is useful for approximating the scattering from very small objects such as rain and snow for weather radar and satellite propagation through the earth's atmosphere.

In the high frequency limit, the electromagnetic field in the shadow zone near to the body is zero for a perfectly conducting body. Non-zero fields are generally experimentally observed in this shadow zone [Bow69], [Stu81]. In addition, the exact solutions of many problems such as the scattering by an infinite half-plane contain non-zero fields in the shadow zone [Som54]. Hence, there must be another mode of propagation. Diffraction theory explains the existence of a non-zero field in this shadow region. The diffraction fields are defined as the difference between the fields as determined by the exact solution and the fields predicted in the high frequency limit. The diffraction field is most significant near the boundary between the lit region and the shadow zone. In order to accurately determine the fields in the shadow region and near the boundaries of the lit and shadow regions, diffraction must be considered.

When a portion of a scattering body is concave, multiple scattering can occur. Figure 4.1-4 shows a concave body. Notice that in the concave portion of the body, an impinging field represented by a ray can be scattered off the body to another part of the 


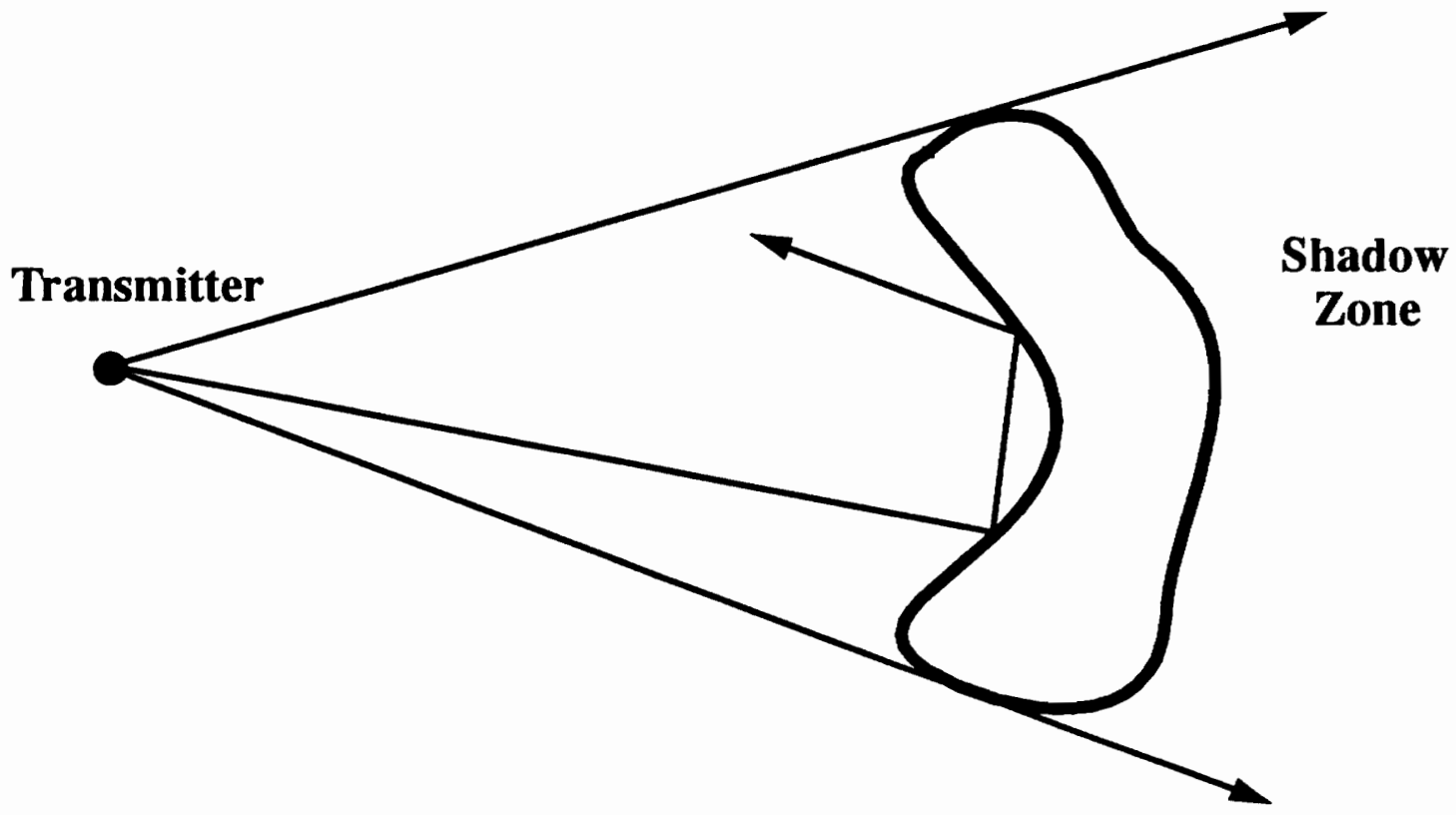

FIGURE 4.1-4. Concave scatterer that induces multiple scattering from a single scattering object.

body. This indicates multiple scattering. Problems that involve multiple scattering by concave bodies are generally difficult to solve.

\subsection{Geometrical Optics [Bor65], [Des72], [Col85], [Van85]}

When the wavelength of a propagating electromagnetic wave is very small, a reasonable approximation to the propagation laws is obtained when the finiteness of the wavelength is neglected. Neglecting the wavelength corresponds to the limiting case of $\lambda_{0} \rightarrow 0$. This approximation is known as geometrical optics since the language of geometry is used to formulate the optical laws. The difference between the true solution and the high-frequency geometrical optics solution is accounted for by diffraction theory. The validity of a geometrical optics model will depend on the size of scattering objects relative to a wavelength. For sufficiently high frequencies, geometrical optics fields may dominate the scattering, and may not require any corrections [Bal89]. 
The ratio of the magnitudes of the far fields (local plane waves) of a propagating electromagnetic wave in a vacuum are related by the intrinsic impedance of the vacuum (essentially free space). This intrinsic impedance is defined in equation (4.2-1).

$$
\frac{|\vec{E}|}{|\vec{H}|}=\eta_{0}=\sqrt{\frac{\mu_{0}}{\varepsilon_{0}}}=377 \Omega
$$

In a homogeneous medium, $\frac{|\vec{E}|}{|\vec{H}|}=\eta=\sqrt{\frac{\mu}{\varepsilon+\frac{\sigma}{j \omega}}}$

TABLE 4.2-1: Table of variables and descriptions for geometrical optics.

\begin{tabular}{|c|c|}
\hline Variable: & Description [units] \\
\hline$\vec{E}$ & Electric field intensity vector $[\mathrm{V} / \mathrm{m}]$ \\
\hline$\vec{H}$ & Magnetic field intensity vector [A/m] \\
\hline$\mu_{0}$ & Permeability of free space $[\mathrm{H} / \mathrm{m}]$ \\
\hline$\varepsilon_{0}$ & Permittivity of free space $[\mathrm{F} / \mathrm{m}]$ \\
\hline$\eta_{0}$ & Impedance of free space $[\Omega]$ \\
\hline$\mu$ & Permeability of the medium $[\mathrm{H} / \mathrm{m}]$ \\
\hline$\varepsilon$ & Permittivity of the medium $[\mathrm{F} / \mathrm{m}]$ \\
\hline$\sigma$ & Conductivity of the medium [S/m] \\
\hline$\omega$ & Radian frequency [radians/second] \\
\hline$\eta$ & Impedance of the medium $[\Omega]$ \\
\hline$\vec{\alpha}$ & Vector magnitude of geometrical optics electric field [V/m] \\
\hline$\vec{\beta}$ & Vector magnitude of geometrical optics magnetic field $[\mathrm{A} / \mathrm{m}]$ \\
\hline $\mathbf{k}_{0}$ & Free space wavenumber [radians/m] \\
\hline$\lambda_{0}$ & Free space carrier wavelength [meters] \\
\hline$L(x, y, z)$ & Geometrical optics field phase function [meters] \\
\hline$n=\sqrt{\mu_{r} \varepsilon_{r}}$ & Index of refraction of the medium \\
\hline
\end{tabular}

Table 4.2-1 describes the variables used here to describe geometrical optics fields. Since the ratio of the magnitudes of the $\vec{E}$ and $\vec{H}$ fields is constant, the phase difference 
between the two fields must be constant in the far field. According to the SommerfeldRunge procedure, this property is assumed valid everywhere outside the sources and $\vec{E}$ and $\vec{H}$ are of the form [Bor65], [Van85]

$$
\begin{aligned}
& \vec{E}=\vec{\alpha}(x, y, z) e^{-j k_{0} L(x, y, z)} \\
& \vec{H}=\vec{\beta}(x, y, z) e^{-j k_{0} L(x, y, z)}
\end{aligned}
$$

Note that the phase function $L(x, y, z)$ is the same for both the electric and magnetic fields. Although the fields vary rapidly as $k_{0} \rightarrow \infty$, the geometrical optics assumption is that $\vec{\alpha}, \vec{\beta}$, and $L$ remain bounded as $k_{0} \rightarrow \infty$, and do not vary rapidly over a distance on the order of $\lambda_{0}[\operatorname{Van} 85]$.

Geometrical optics attempts to determine the wavefronts (equi-phase surfaces) given by $L(x, y, z)=$ constant. These surfaces are called the geometrical wavefronts. Optical theory, which is the high-frequency limit, considers the propagation of a wave to be the progression of the wavefronts into a succession of contiguous ones [Van85]. The orthogonal trajectories of the wavefronts can be represented by rays that point in the direction of the wave propagation. Thus, the term ray tracing corresponds to the tracing of wave propagation in a direction represented by a ray. To within the accuracy of geometrical optics, the propagation velocity is the rate of wave displacement in the ray direction. The ray directions are given by Poynting's vector in isotropic, lossless media. A set of neighboring rays forms a beam (tube of rays) with a variable cross-section as shown in Figure 4.2-1. The intensity $I\left(\mathrm{~W} / \mathrm{m}^{2}\right)$ of the energy transported along a tube containing a finite number of rays is inversely proportional to the wavefront surface area $d A$. Hence, the ray field amplitudes are inversely proportional to the square root of the cross-sectional area of the wavefront surface. This condition is required to conserve the energy in a tube of rays. The inverse proportionality of the energy intensity is demonstrated in Figure 4.2-1 and equation (4.2-5). The distances of the wavefront from the source are denoted by $R_{1}$ and $R_{2}$, and the wavefront areas are labeled $d A_{1}$ and $d A_{2}$. 


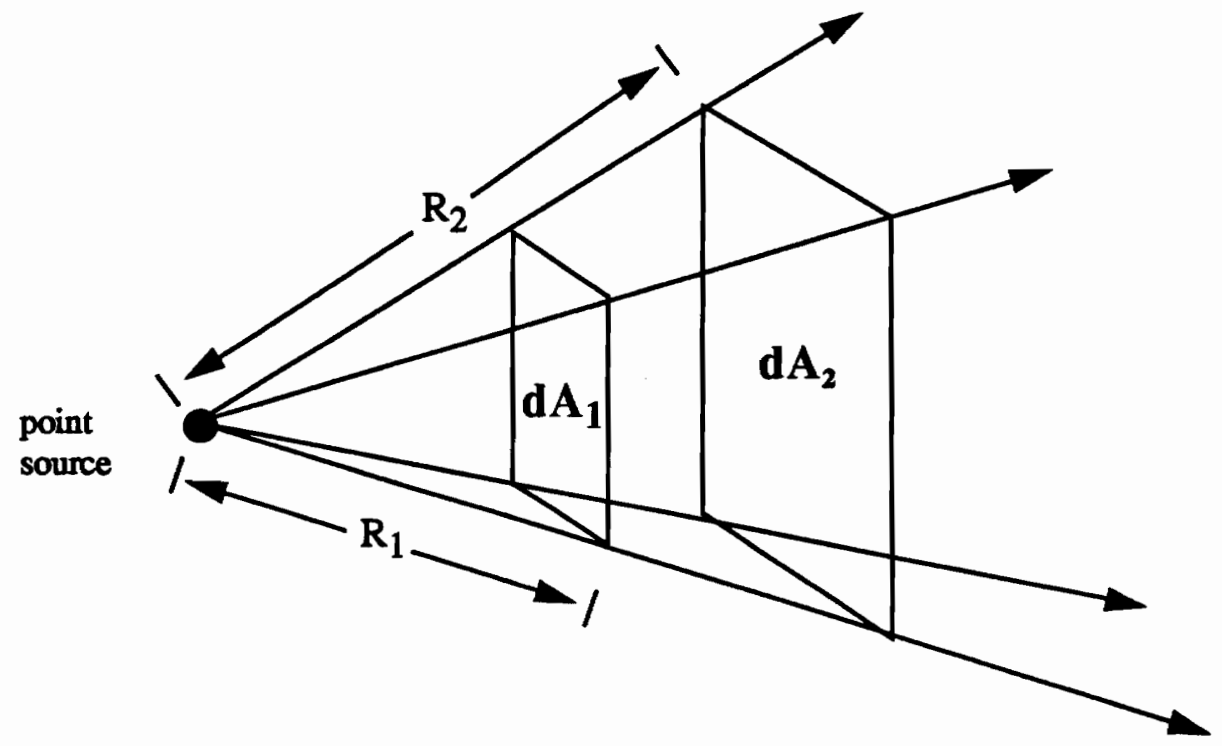

FIGURE 4.2-1. A tube of rays diverging from a point source.

$$
\frac{I_{2}}{I_{1}}=\frac{d A_{1}}{d A_{2}}=\frac{R_{1}^{2}}{R_{2}^{2}}
$$

The simplicity of the geometrical optics model is due to the locally plane wave behavior of the fields at the wavefront represented by each ray.

\subsubsection{Properties of the Geometrical Optics Fields}

- The phase function $L(x, y, z)$ satisfies the eikonal equation where $n=\sqrt{\mu_{r} \varepsilon}$ is the index of refraction in the medium. The velocity of propagation is $1 / n$.

$$
|\nabla L|^{2}=\left(\frac{\partial L}{\partial x}\right)^{2}+\left(\frac{\partial L}{\partial y}\right)^{2}+\left(\frac{\partial L}{\partial z}\right)^{2}=n^{2}(x, y, z)
$$

- The optical path length along a curve joining two points $P_{1}$ and $P_{2}$ satisfies Fermat's Principle. Fermat's Principle states that the actual optical ray path between $P_{1}$ and $P_{2}$ $P_{2}$

$\int n|d c|$ is shorter than the optical path length of any other curve which joins these $P_{1}$

points and which lies in a certain regular neighborhood of it. This implies that ray paths are rectilinear in homogeneous media. 
- At the surface of separation between two homogeneous media, each incident ray creates a reflected ray and a refracted ray. Snell's laws of reflection and refraction determine the ray directions as will be shown in Section 4.2.3 Plane Wave Obliquely Incident Upon a Planar Boundary [Che83].

$$
\theta_{r}=\theta_{i} ; n_{1} \sin \theta_{i}=n_{2} \sin \theta_{t}
$$

- In an inhomogeneous medium, the curvature of a ray path is given by equation (4.2-8). The radius of curvature of the ray path is $p$. The index of refraction is $n$, and the unit normal vector that points toward the center of curvature is denoted by $\hat{n}$. The symbol $\nabla$ is the divergence operator.

$$
\frac{1}{\rho}=\hat{n} \cdot \nabla(\log n)=\hat{n} \cdot \frac{\nabla n}{n},(\rho>0)
$$

\subsubsection{Free Space Path Loss [Stu81], [Col85], [Gri87]}

The path loss dependence with distance of each ray in a geometrical optics model is the same as in free space. Consider a transmitter antenna and a receiver antenna in free space as shown in Figure 4.2-2. The variables used to describe the figure are given in Table 4.2-2. In free space, electromagnetic waves propagate in straight lines.

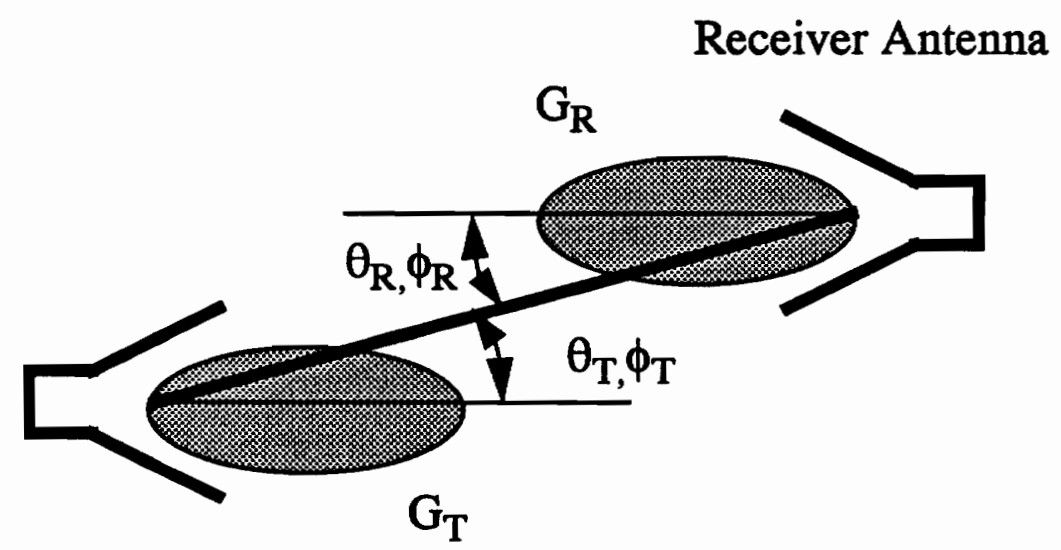

Transmitter Antenna

FIGURE 4.2-2. Transmitter and receiver antenna located in free space (from [Col85]). 
TABLE 4.2-2: Table of variables and definitions for the description of free space propagation.

\begin{tabular}{|c|c|}
\hline Yariable: & Derinition (winis] \\
\hline$\theta_{T}, \phi_{T}$ & Direction of receiver from transmitter relative to antenna boresight \\
\hline$\theta_{R}, \phi_{R}$ & Direction of transmitter from receiver relative to antenna boresight \\
\hline$G_{T}\left(\theta_{T}, \phi_{T}\right)$ & Transmitter antenna gain \\
\hline$G_{R}\left(\theta_{R}, \phi_{R}\right)$ & Receiver antenna gain \\
\hline$P_{T}$ & Transmitted power [Watts] \\
\hline$P_{R}$ & Received Power [Watts] \\
\hline$P L$ & Path loss between transmitter and receiver [dB] \\
\hline$P_{\text {inc }}$ & Power density per unit area incident upon the receiver antenna $\left[\mathrm{W} / \mathrm{m}^{2}\right]$ \\
\hline $\mathbf{r}$ & Distance between transmitter and receiver [m] \\
\hline$A_{e}$ & Effective area of receiver antenna $\left[\mathrm{m}^{2}\right]$ \\
\hline$\lambda$ & Carrier wavelength [m] \\
\hline
\end{tabular}

The transmitter antenna has a power gain of $G_{T}\left(\theta_{T}, \phi_{T}\right)$ in the direction of the receiver. Similarly, the power gain of the receiver antenna is $G_{R}\left(\theta_{R}, \phi_{R}\right)$ in the direction of the transmitter. The power density per unit area incident upon the receiver at a distance $r$ from the transmitter is

$$
P_{i n c}=\frac{P_{T} G_{T}\left(\theta_{T}, \phi_{T}\right)}{4 \pi r^{2}}
$$

for a transmitted power $P_{T}$. The effective area of an antenna $\left(A_{e}\right)$ is a measure of the ability of an antenna to collect power from an incident wave and deliver it to its terminals [Stu81].

$$
A_{e}=G_{R}\left(\theta_{R}, \phi_{R}\right) \frac{\lambda^{2}}{4 \pi}
$$


The power captured by the receiver antenna is the incident power density $\left(P_{\text {inc }}\right)$ times the effective area of the antenna. Hence, the received power is

$$
P_{R}=P_{T} G_{T}\left(\theta_{T}, \phi_{T}\right) G_{R}\left(\theta_{R}, \phi_{R}\right)\left(\frac{\lambda}{4 \pi r}\right)^{2}
$$

or

$P_{R}[\mathrm{dBm}]=$

$$
P_{T}[\mathrm{dBm}]+G_{T}[\mathrm{dBi}]+G_{R}[\mathrm{dBi}]+20 \times \log _{10}\left(\frac{\lambda}{4 \pi r}\right)
$$

Path loss is defined as the difference between the transmitted and received power.

$$
P L[\mathrm{~dB}]=P_{T}[\mathrm{dBm}]-P_{R}[\mathrm{dBm}]
$$

Comparing equations (4.2-12) and (4.2-13), the path loss between two antennas located in free space is given by

$$
P L(r)[\mathrm{dB}]=20 \times \log _{10}\left(\frac{4 \pi r}{\lambda}\right)-G_{T}[\mathrm{dBi}]-G_{R}[\mathrm{dBi}]
$$

which is the Friis free space transmission equation. Note that equation (4.2-14) is independent of $P_{T}$ since free space is linear for field strength less than $3 \mathrm{kV} / \mathrm{mm}$ at atmospheric pressure. Equation (4.2-14) is the true free space path loss between two antennas. If path loss is referenced to isotropic antennas $\left(G_{T}=G_{R}=1=0 \mathrm{dBi}\right)$, then

$$
P L(r)[\mathrm{dB}]=20 \times \log _{10}\left(\frac{4 \pi r}{\lambda}\right)
$$

Referencing the path loss to isotropic antennas is equivalent to determining the path loss due strictly to the radio channel without influence from the measurement antenna pattern. 


\subsubsection{Plane Wave Obliquely Incident Upon a Planar Boundary [Che83]}

In free space, electromagnetic waves travel in straight paths. However, radio waves must propagate in regions that are not entirely free space. This is true in buildings where planar objects such as walls and partitions are common. Thus, it is important to understand the interaction of plane waves and planar surfaces. Consider a uniform plane wave with perpendicular (TE) polarization obliquely incident upon a planar dielectric boundary shown in Figure 4.2-3. The polarization is perpendicular because the $\vec{E}$ field is perpendicular (coming out of the page) to the plane of incidence. The plane of incidence is the plane that contains the vectors $\hat{k}_{i}$ and $\hat{n}$. Note that the entire region below the boundary is made up of the dielectric material and that the interface extends infinitely in the positive and negative $z$ direction. Table 4.2-3 summarizes the variables used to describe the fields in equations (4.2-16) through (4.2-23). A detailed textbook derivation of the Fresnel plane wave reflection and transmission coefficients is given in Appendix A.

Perpendicular (TE) Polarization

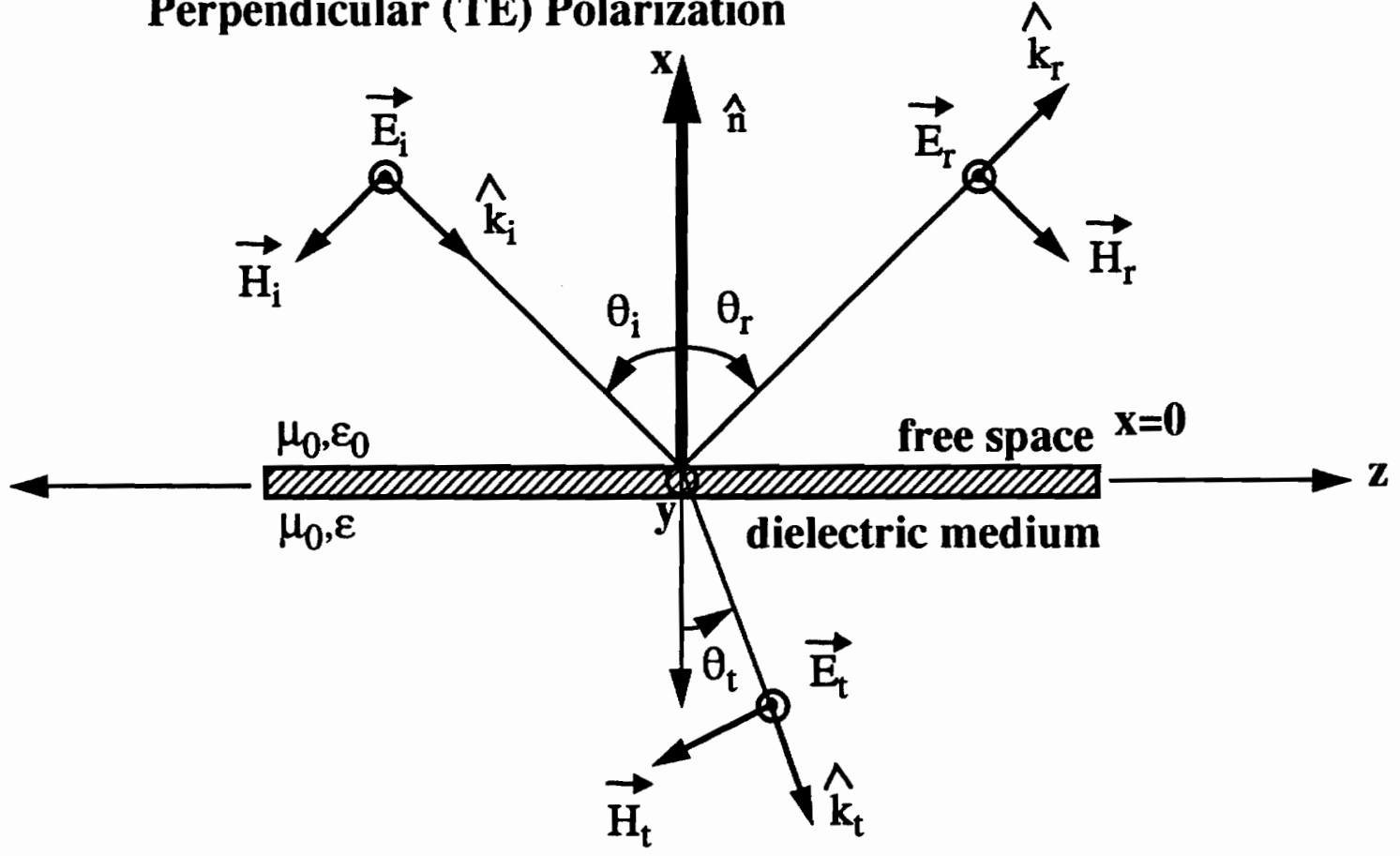

FIGURE 4.2-3. Reflection and transmission field polarization of a perpendicularly (horizontally) polarized plane wave obliquely incident upon a planar dielectric boundary. Note that the entire region below the boundary is made up of the dielectric material and that the interface extends infinitely in the positive and negative z direction. 
TABLE 4.2-3: Summary of variables used to describe plane wave reflection in equations (4.2-16) through (4.2-23).

\begin{tabular}{|c|c|}
\hline Viriable: & Decription lumist) \\
\hline$\mu_{0}$ & Permeability of free space $[\mathrm{H} / \mathrm{m}]$ \\
\hline$\varepsilon_{0}$ & Permittivity of free space $[\mathrm{F} / \mathrm{m}]$ \\
\hline$\varepsilon=\varepsilon_{r} \varepsilon_{0}$ & Permittivity of dielectric material [F/m] \\
\hline$\sigma$ & Conductivity of dielectric material [S/m] \\
\hline$\varepsilon_{r}^{*}$ & Complex relative dielectric constant of material \\
\hline$\theta_{\mathrm{i}}$ & Angle of incidence (measured from surface normal) [radians] \\
\hline$\vec{E}_{i}$ & Incident electric field [V/m] \\
\hline$\vec{H}_{i}$ & Incident magnetic field $[\mathrm{A} / \mathrm{m}]$ \\
\hline$\hat{k}_{i}$ & Propagation direction of incident field \\
\hline$\theta_{\mathrm{r}}$ & Angle of reflection (measured from surface normal) [radians] \\
\hline$\vec{E}_{r}$ & Reflected electric field $[\mathrm{V} / \mathrm{m}]$ \\
\hline$\vec{H}_{r}$ & Reflected magnetic field [A/m] \\
\hline$\hat{k}_{r}$ & Propagation direction of reflected field \\
\hline$\theta_{t}$ & Angle of transmission (measured from surface normal) [radians] \\
\hline$\vec{E}_{t}$ & Transmitted electric field $[\mathrm{V} / \mathrm{m}]$ \\
\hline$\vec{H}_{t}$ & Transmitted magnetic field $[\mathrm{A} / \mathrm{m}]$ \\
\hline$\vec{k}_{t}$ & Propagation direction of transmitted field \\
\hline$\eta=\sqrt{\mu / \varepsilon}$ & Wave impedance in dielectric medium $[\Omega]$ \\
\hline$\eta_{0}$ & Wave impedance in free space $[\Omega]$ \\
\hline$k=2 \pi / \lambda=\omega \sqrt{\mu \varepsilon}$ & Wave number in dielectric medium [radians/m] \\
\hline$n_{2}=\sqrt{\varepsilon_{r}}$ & Index of refraction in dielectric medium \\
\hline$\hat{x}$ & Unit vector in the coordinate direction $x$ \\
\hline
\end{tabular}




\begin{tabular}{|c|c|}
\hline Varibile & Oescription [units \\
\hline$\hat{y}$ & Unit vector in the coordinate direction y \\
\hline$\hat{z}$ & Unit vector in the coordinate direction $\mathrm{z}$ \\
\hline$\Gamma_{\perp}$ & Reflection coefficient for perpendicular polarization \\
\hline$T_{\perp}$ & Transmission coefficient for perpendicular polarization \\
\hline$\Gamma_{\|}$ & Reflection coefficient for parallel polarization \\
\hline$T_{\|}$ & Transmission coefficient for parallel polarization \\
\hline
\end{tabular}

In order to satisfy Maxwell's equations, the tangential components of the $\vec{E}$ and $\vec{H}$ fields must be continuous at the boundary. Snell's laws of reflection and refraction give

$$
\theta_{r}=\theta_{i} ; \frac{\sin \theta_{t}}{\sin \theta_{i}}=\frac{k_{0}}{k}=\frac{\omega \sqrt{\mu_{0} \varepsilon_{0}}}{\omega \sqrt{\mu_{0} \varepsilon}}=\frac{1}{\sqrt{\varepsilon_{r}}}=\frac{1}{n_{2}}
$$

Solving for the reflected and transmitted field amplitudes in terms of the incident field amplitudes gives the Fresnel reflection and transmission coefficients for perpendicular (TE) polarization. The reflection $\left(\Gamma_{\perp}\right)$ and transmission $\left(T_{\perp}\right)$ coefficients are given as

$$
\begin{aligned}
& \Gamma_{\perp}=\frac{E_{\text {ro }}}{E_{\text {io }}}=\frac{\left(\eta / \cos \theta_{t}\right)-\left(\eta_{0} / \cos \theta_{i}\right)}{\left(\eta / \cos \theta_{t}\right)+\left(\eta_{0} / \cos \theta_{i}\right)} \\
& T_{\perp}=\frac{E_{t o}}{E_{\text {io }}}=\frac{2\left(\eta / \cos \theta_{t}\right)}{\left(\eta / \cos \theta_{t}\right)+\left(\eta_{0} / \cos \theta_{i}\right)}
\end{aligned}
$$

The reflection coefficient $\Gamma_{\perp}$ and the transmission coefficient $T_{\perp}$ are related as follows.

$$
1+\Gamma_{\perp}=T_{\perp}
$$

A similar analysis follows for parallel (TM) polarization shown in Figure 4.2-4. 
Parallel (TM) Polarization

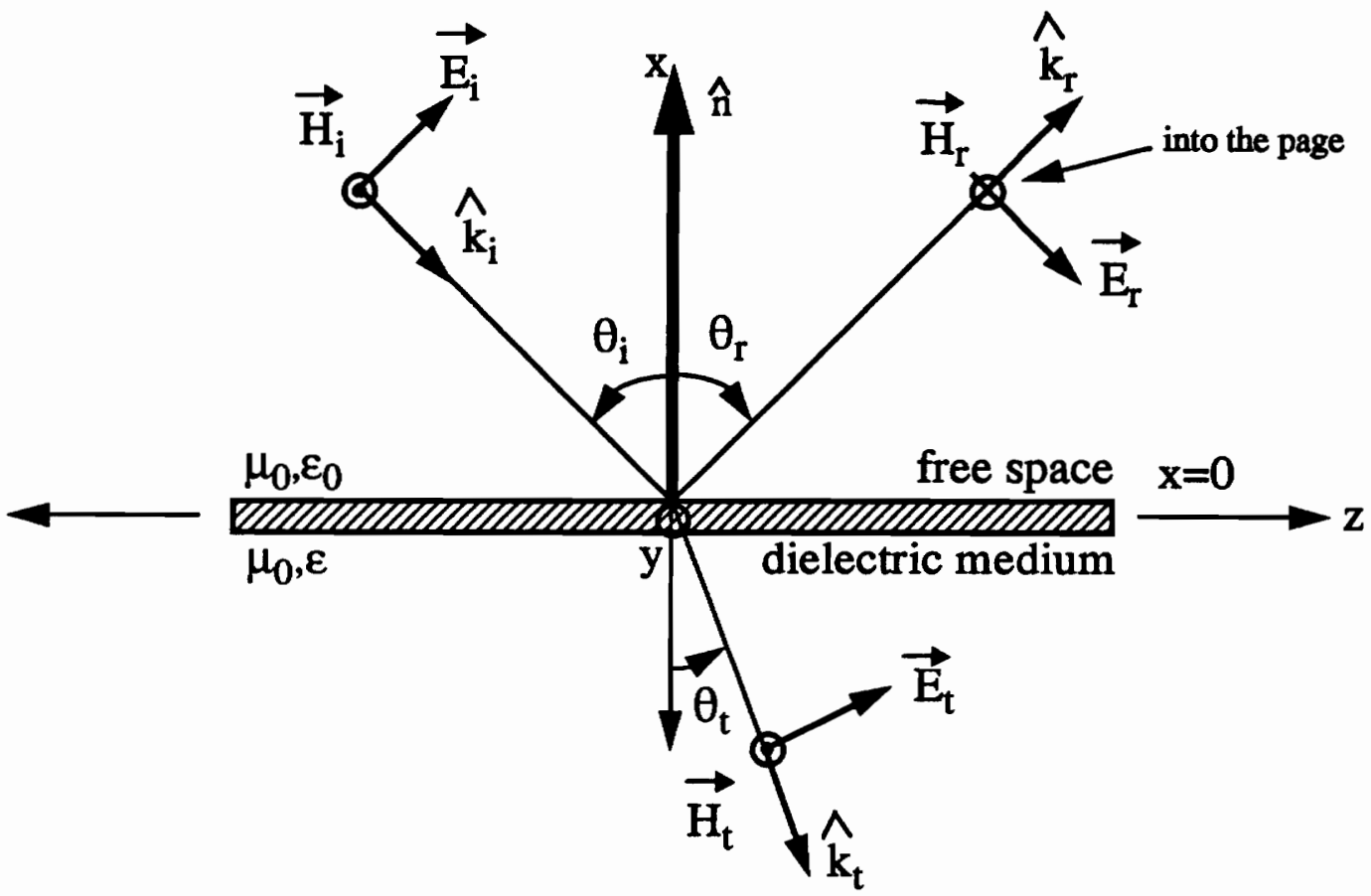

FIGURE 4.2-4. Reflection and transmission field polarization of a vertically polarized plane wave incident upon a planar dielectric boundary. Note that the entire surface below the interface is made up of the dielectric medium and that the surface boundary extends to positive and negative infinity in the $z$ direction.

$$
\begin{gathered}
\Gamma_{\|}=\frac{E_{r o}}{E_{i o}}=\frac{\left(\eta \cos \theta_{t}-\eta_{0} \cos \theta_{i}\right)}{\left(\eta \cos \theta_{t}+\eta_{0} \cos \theta_{i}\right)} \\
T_{\|}=\frac{E_{t o}}{E_{i o}}=\frac{2 \eta \cos \theta_{i}}{\left(\eta \cos \theta_{t}+\eta_{0} \cos \theta_{i}\right)} \\
1+\Gamma_{\|}=T_{\|}\left(\frac{\cos \theta_{t}}{\cos \theta_{i}}\right)
\end{gathered}
$$

Lossy materials may be considered by letting the relative permittivity of the dielectric constant be complex. The term $\frac{\sigma}{\omega \varepsilon}$ is referred to as the loss tangent.

$$
\varepsilon_{r}^{*}=\varepsilon_{r}\left(1-j \frac{\sigma}{\omega \varepsilon}\right)
$$


Polarizations other than vertical and horizontal may be divided into vertically and horizontally polarized components [Bec68]. Conductivity, permittivity, frequency, and angle of incidence influence the Fresnel reflection coefficients. Table 4.2-4 gives the loss tangents and relative dielectric constants for dry concrete over a frequency range of $1 \mathrm{kHz}$ to $10 \mathrm{GHz}$ (from [Von54]).

TABLE 4.2-4: Table of loss tangent and relative permittivity for dry cement over a frequency range of $1 \mathrm{kHz}$ to $10 \mathrm{GHz}$.

\begin{tabular}{|c|c|c|}
\hline Irequency & 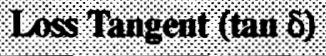 & Relative Peraitivity (8) \\
\hline $1 \mathrm{kHz}$ & $35.5 \times 10^{-4}$ & 2.48 \\
\hline $10 \mathrm{kHz}$ & $28.9 \times 10^{-4}$ & 2.48 \\
\hline $100 \mathrm{kHz}$ & $25.9 \times 10^{-4}$ & 2.48 \\
\hline $1 \mathrm{MHz}$ & $25.5 \times 10^{-4}$ & 2.48 \\
\hline $10 \mathrm{MHz}$ & $22.2 \times 10^{-4}$ & 2.48 \\
\hline $100 \mathrm{MHz}$ & $15.0 \times 10^{-4}$ & 2.47 \\
\hline $3 \mathrm{GHz}$ & $7.8 \times 10^{-4}$ & 2.40 \\
\hline $10 \mathrm{GHz}$ & $6.8 \times 10^{-4}$ & 2.35 \\
\hline
\end{tabular}

Plots of the magnitudes of the Fresnel transmission and reflection coefficients for plicene cement and marble at $4.0 \mathrm{GHz}$ are shown in Figure 4.2-5 as a function of incidence angle for both vertical and horizontal polarization. The incidence angle is measured from the surface normal. The relative dielectric constant of the plicene cement is 2.4 , and the relative dielectric constant for the marble is 8.8 as given in [Von54]. The loss tangent of the cement is $7.8 \times 10^{-4}$, and the loss tangent of the marble is $2.5 \times 10^{-2}$. The magnitudes of the reflection and transmission coefficients for these two materials are plotted since they are two likely building materials that have different dielectric properties. Notice that the magnitude of the vertical polarization reflection coefficient for plicene cement goes through a minimum value for an incidence angle of 57 degrees. The angle where the magnitude of the reflection coefficient is a minimum is called the Brewster angle. As the reflection coefficient goes through the minimum value with decreasing values of grazing angle, the phase changes rapidly from around zero degrees to 180 degrees. Thus, the reflection coefficient is nearly -1 for small grazing angles and vertical polarization. The horizontal reflection coefficient is also nearly -1 for small grazing angles. This limiting 
value is independent of the dielectric constant of the lower medium. For the higher dielectric constant marble, the Brewster angle is increased to 71 degrees and the reflection coefficients at a given incidence angle are larger than for the cement. The transmission coefficients at a given incidence angle are smaller than the transmission coefficients of the cement.
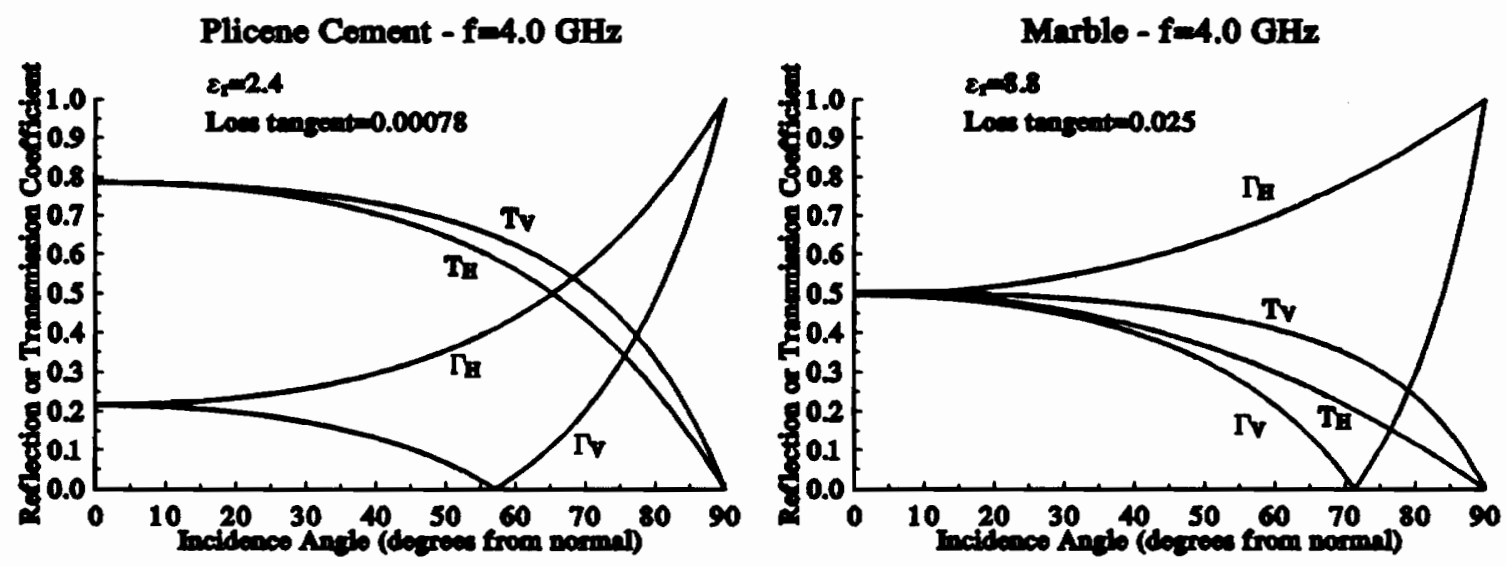

FIGURE 4.2-5. Magnitude of the reflection and transmission coefficient of plicene cement and marble for both vertical and horizontal polarization at $4.0 \mathrm{GHz}$. The dielectric properties are found in [Von54].

An alternative derivation of the reflection coefficients can be determined by the principle of equivalence. Equivalent surface currents on the boundary of the two media can be determined from the boundary conditions on the total field. These surface currents exist everywhere on the surface and generate the specular scattered fields given above. When the surface is no longer infinite, the scattering pattern changes. The effect of a finite size scatterer is examined in Section 4.6 Computed Scattering and Diffraction Patterns using Physical Optics, and the Geometrical Theory of Diffraction since real scattering objects are never infinite in extent.

Reflection coefficient models are generally valid when objects are smooth surfaces and the smallest dimension of the object is greater than 50 [Par89]. The Rayleigh parameter $(\Sigma)$ may be used to judge whether a surface is smooth or rough relative to the wavelength [Par89], where 


$$
\Sigma=\frac{4 \pi \sigma}{\lambda} \sin \theta
$$

The standard deviation of the surface roughness relative to the mean height of the surface is $\sigma, \lambda$ is the carrier wavelength, and $\theta$ is the incidence angle measured from grazing incidence. It is suggested that if $\Sigma<0.1$, the surface can be considered smooth and the reflection coefficient model is valid. For $\Sigma>10$, the reflected wave is almost insignificant [Par89]. The impulse response of a rough surface is given in [Bro77] for the incoherent scattered power.

\subsubsection{Plane-Earth Reflection Path Loss Model [Col85]}

Geometrical optics can be used to predict path loss when two antennas are located above a flat earth as indicated in Figure 4.2-6. The transmitter and receiver antennas are at heights $h_{1}$ and $h_{2}$ above the ground, and are separated by $d$ meters. The variables used to describe the figure are given in Table 4.2-5.

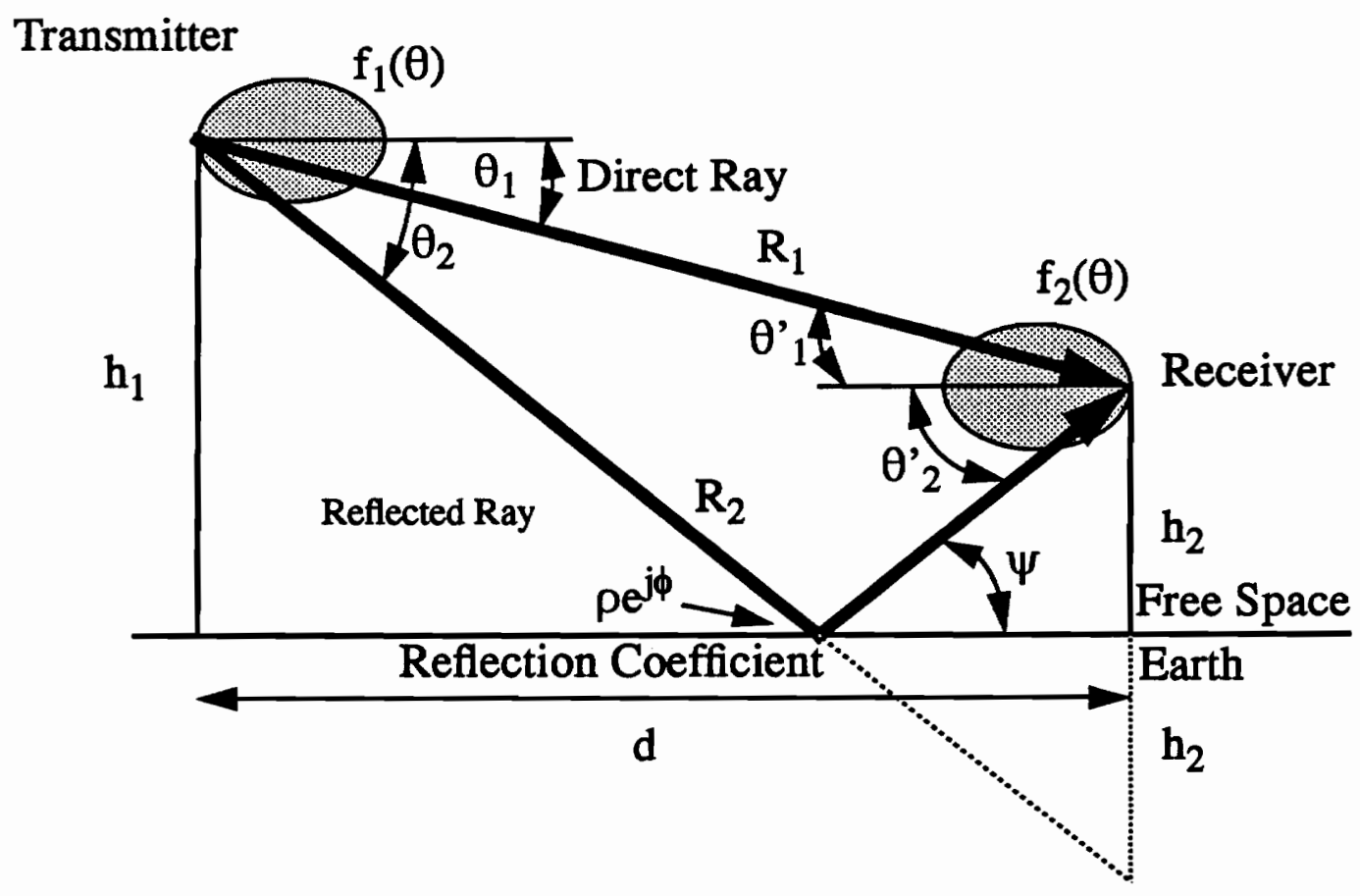

FIGURE 4.2-6. Pictoral representation of plane-Earth reflection. 
TABLE 4.2-5: Table of variables and descriptions used to describe the two-ray planeEarth reflection model.

\begin{tabular}{|c|c|}
\hline Yarable & 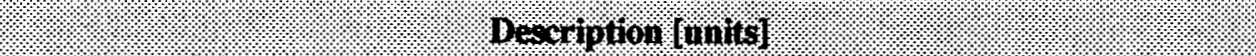 \\
\hline$h_{1}$ & Transmitter antenna beight [meters] \\
\hline $\mathbf{h}_{2}$ & Receiver antenna height [meters] \\
\hline d & Ground separation between transmitter and receiver antennas [meters] \\
\hline $\mathbf{R}_{\mathbf{1}}$ & Path length of direct ray [meters] \\
\hline $\mathbf{R}_{\mathbf{2}}$ & Path length of reflected ray [meters] \\
\hline$\theta$ & Direction of ray path relative to antenna pattern [radians] \\
\hline$f_{1}\left(\theta_{1}\right)$ & Radiation field strength pattern of transmitter antenna in the direction of the receiver \\
\hline$f_{2}\left(\theta^{\prime}{ }_{1}\right)$ & Radiation field strength pattern of receiver antenna in the direction of the transmitter \\
\hline$f_{1}\left(\theta_{2}\right)$ & Radiation field strength pattern of transmitter antenna in the direction of the reflected ray \\
\hline$f_{2}\left(\theta_{2}^{\prime}\right)$ & Radiation field strength pattern of receiver antenna in the direction of the reflected ray \\
\hline $\mathrm{V}_{1}$ & Voltage induced at receiving antenna due to the direct ray [Volts] \\
\hline $\mathrm{V}_{2}$ & Voltage induced at receiving antenna due to the reflected ray [Volts] \\
\hline $\mathbf{k}_{\mathbf{0}}$ & Free space wavenumber, $\mathrm{k}_{0}=2 \pi / \lambda, \lambda$ is the carrier wavelength [radians $/ \mathrm{m}$ ] \\
\hline$p e^{j \phi}$ & Reflection coefficient for the ground \\
\hline
\end{tabular}

Both the direct and specularly reflected ground ray that reach the receiver are shown in Figure 4.2-6. The direct and reflected ray can combine constructively or destructively according to the path lengths $R_{1}$ and $R_{2}$ and the phase of the reflection coefficient. Thus, the coherent sum of the fields must be used to compute the received power. The field of the direct ray produces a voltage at the receiving antenna that is proportional to

$$
V_{1} \propto f_{1}\left(\theta_{1}\right) f_{2}\left(\theta^{\prime}\right) \frac{e^{-j k_{0} R_{1}}}{4 \pi R_{1}}
$$

where $f_{1}(\theta)$ and $f_{2}(\theta)$ are the radiation field strength patterns of the two antennas.

$$
f_{1}(\theta)=\sqrt{G_{T}(\theta)}, f_{2}(\theta)=\sqrt{G_{R}(\theta)}
$$


The indirect reflected ray produces a voltage proportional to

$$
V_{2} \propto f_{1}\left(\theta_{2}\right) f_{2}\left(\theta_{2}^{\prime}\right) \frac{e^{-j k_{0} R_{2}}}{4 \pi R_{2}}
$$

The total received voltage is proportional to $\left|V_{1}+V_{2}\right|$ where $V_{1}$ and $V_{2}$ are complex. The total received power is proportional to

$$
P_{R} \propto 20 \times \log _{10}\left|V_{1}+V_{2}\right|
$$

Consider the behavior of the received power as a receiver moves radially away from the transmitter. Figure 4.2-7 shows the received power relative to the free space power received at a 10 meter separation distance for $f=900 \mathrm{MHz}$ and antenna heights of 10 $\mathrm{m}$ and $2 \mathrm{~m}$. For this numerical example, the reflection coefficient $p e^{j \phi}$ is assumed to be -1 for all angles of incidence and reflection. Although this is not true in practice, it is valid for small grazing angles which is the region of interest. Notice that up to distances of 200 meters, the received power undergoes many rapid fluctuations as the receiver is moved. The average received power (spatial average of the instantaneous signal) decreases nearly as in free space ( $20 \mathrm{~dB} / \mathrm{dec} a d e)$. At distances greater than 200 meters, received power decreases as $40 \mathrm{~dB} /$ decade.

The rapid fluctuations for $\mathrm{d}<200 \mathrm{~m}$ are caused by the relative change of the phase for the individual multipath components as the path lengths $R_{1}$ and $R_{2}$ approach each other. For $d>200 \mathrm{~m}, R_{1}$ and $R_{2}$ are nearly equal and the path lengths differ by less than half a wavelength. Due to the $180^{\circ}$ phase shift at the ground, the two voltages nearly cancel. The asymptotic behavior $\left(d>h_{t} h_{r}\right)$ of the received power is [Par89]

$$
\frac{P_{R}}{P_{T}}=G_{R} G_{T}\left(\frac{h_{t} h_{r}}{d^{2}}\right)^{2}
$$

The received power decreases as $d^{4}$ which gives a $40 \mathrm{~dB} /$ decade path loss slope seen in Figure 4.2-7. This model has been used to estimate the capacity of a CDMA cellular radio system [Rap92]. 


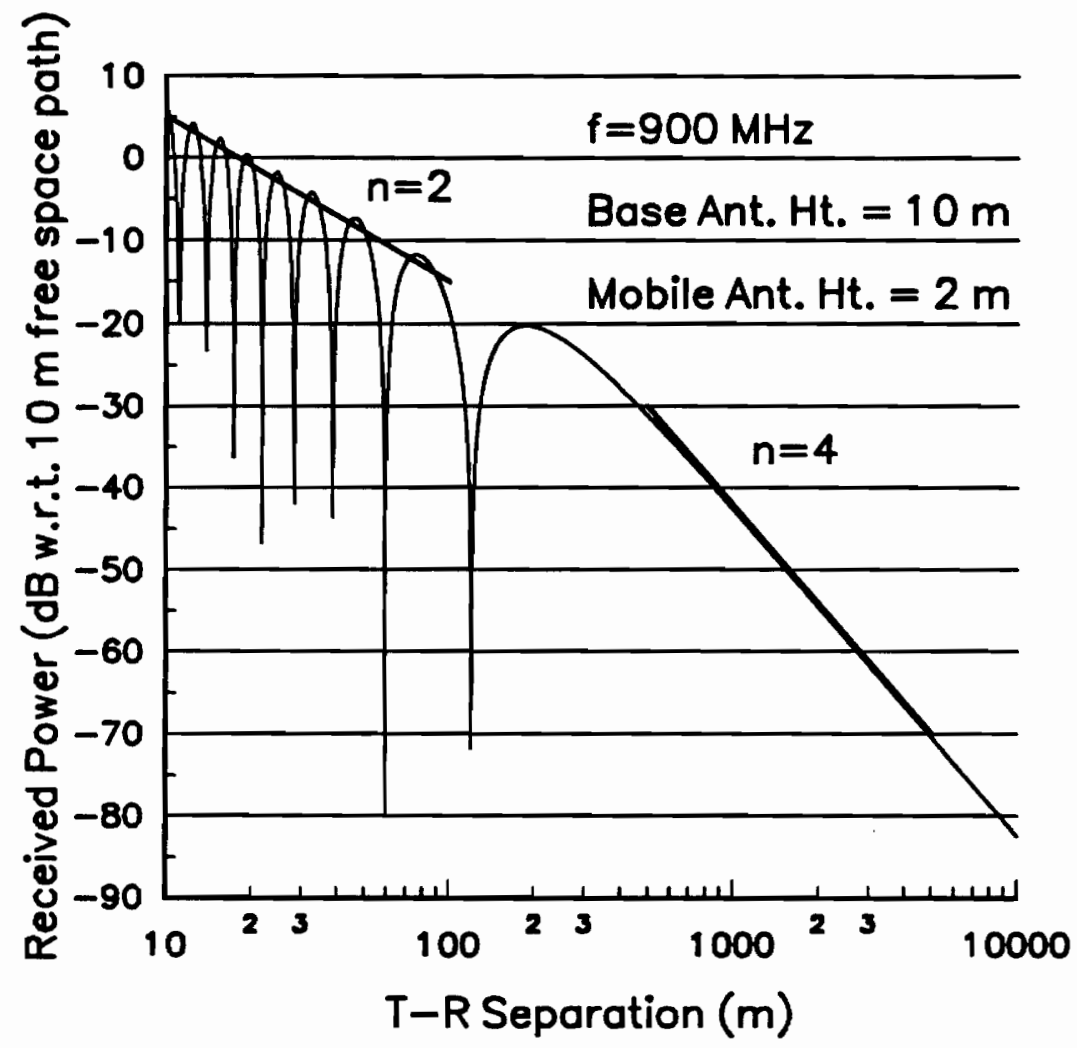

FIGURE 4.2-7. Received signal as the receiver is moved radially away from the transmitter. Received power decreases with distance at a rate of $10 \cdot n \mathrm{~dB} / \mathrm{decade}$.

\subsection{Geometrical Theory of Diffraction [Kel62], [Kou74], [Bal89]}

\subsubsection{Perfectly Conducting Surface}

Geometrical optics fails to account for diffracted energy in the shadow regions, and the geometrical optics field is discontinuous at shadow boundaries. The Geometrical Theory of Diffraction (GTD) supplements geometrical optics by introducing a diffracted field that accounts for the non-zero fields in shadow regions and modifies the field in the GO region so that the total field is continuous. This is important since in a mobile and portable radio environment, the receiver is often shadowed from the transmitter. It is important to be able to predict the changes in the propagation as a receiver moves from an unobstructed to an obstructed location. At high frequencies, diffraction, like reflection and refraction, depends on the geometry of the object and the amplitude, phase, and polariza- 
tion of the incident field at the point of diffraction [Bal89]. Some of the advantages of GTD as given in [Ba189] are listed below.

- It is simple to apply.

- It can be used to solve complicated problems that are not amenable to other methods such as the radar cross-section of complex objects.

- It provides physical insight into the radiation and scattering mechanisms from the various parts of the structure.

- It yields accurate results within its region of validity that compare quite well with experiments and other methods.

The exact solution of high-frequency diffraction problems such as diffraction by an infinite half-plane contain terms that are not included in the geometrical optics solution [Som54]. These terms are diffraction terms that account for the non-zero field in shadow regions. Consider a perfectly conducting infinite wedge in free space as shown in Figure 4.3-1. The wedge is infinite in the dimension perpendicular to the page as well as in the directions indicated in the figure by arrows. An electromagnetic wave is incident upon the wedge from the source point $O$ in the plane that contains the observation point $P$. This plane is the page surface. The received field is observed at a constant radius $\rho$ from the tip of the wedge. Figure 4.3-2 shows the wedge geometry. The cylindrical coordinates $\rho$ and $\phi$ are the observation coordinates, and $\rho$ ' and $\phi$ ' are the source coordinates. The distances are measured from the tip of the wedge, and the angles are measured from the upper wedge face.

Three distinct regions can be seen in Figure 4.3-1 and Figure 4.3-2. The boundaries between the regions are determined by what rays can reach the observation point. In region I, direct, reflected, and diffracted rays are received. The reflection shadow boundary (RSB) is the boundary between where a reflected ray can and cannot be received. This boundary occurs at the angle $\phi=\pi-\phi$ '. In region II, direct and diffracted rays are received, and the incident shadow boundary (ISB) occurs at the angle $\phi=\pi+\phi$ ' as indicated in Figure 4.3-2. The ISB is the boundary between where the incident ray can and cannot be received. The direct and reflected fields are determined according to geometrical optics. A typical reflected field originates at the point $Q_{R}$ on the face of the wedge in Figure 4.3-1. 


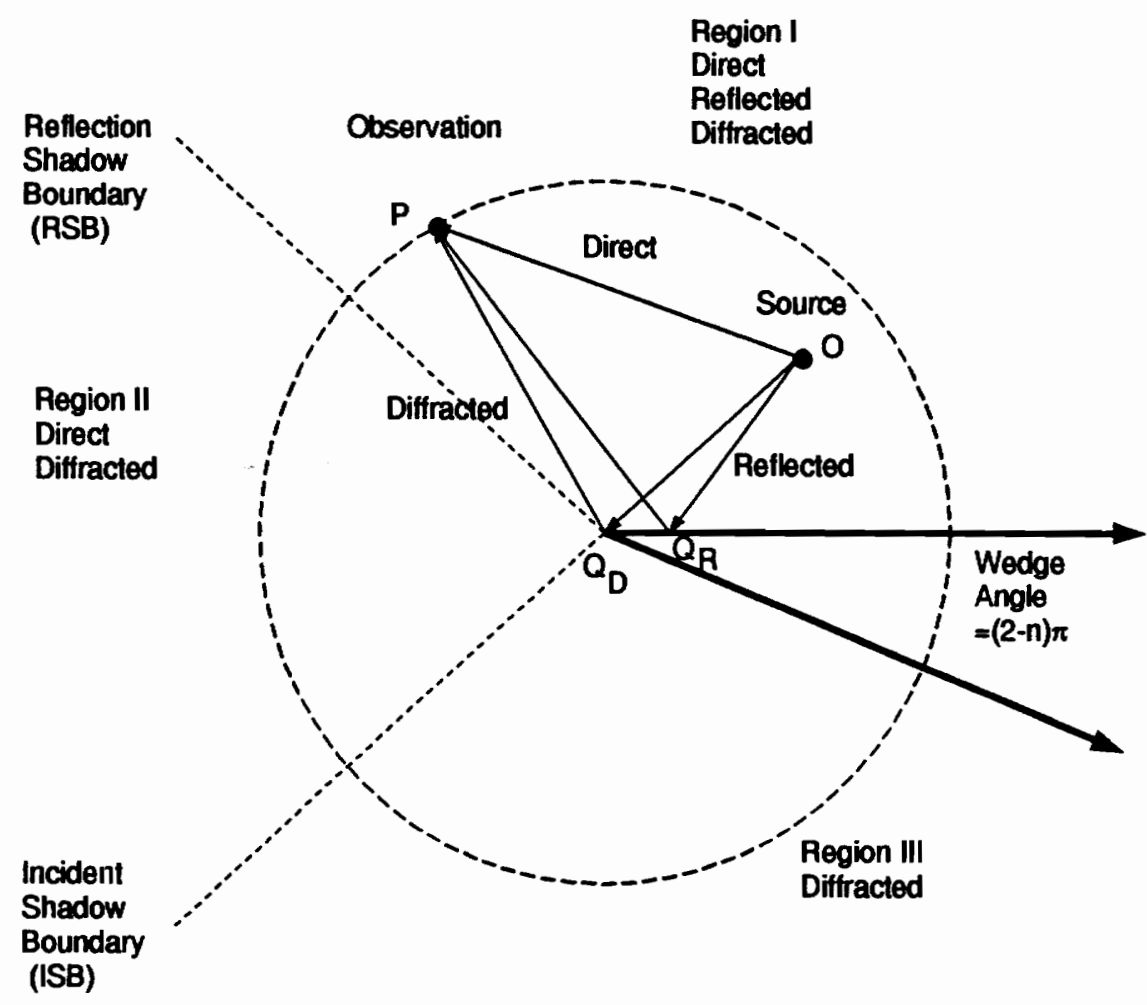

FIGURE 4.3-1. Canonical problem of diffraction by a perfectly conducting wedge of interior angle (2-n) $\pi$ (from [Bal89]). The angles are indicated in Figure 4.3-2. The points $Q_{R}$ and $Q_{D}$ are the points of reflection and diffraction on the wedge.

The diffracted field originates at the tip of the wedge at point $Q_{D}$, and propagates directly to the observation point. The diffracted field can be written as

$$
E_{d}(P)=E_{i}\left(Q_{D}\right) D\left(L, \bar{\phi}, \bar{\phi}^{\prime}, n\right) A\left(\rho^{\prime}, \rho\right) e^{-j k \rho}
$$

where $D$ is a diffraction coefficient that relates the amplitude of the diffracted field to the amplitude of the field incident at the tip of the wedge $E_{i}\left(Q_{D}\right)$ as determined by geometrical optics. In general, the diffraction coefficient depends on observation distance, angle of incidence, angle of diffraction, wedge angle, and wave polarization. The variable $L$ is a distance parameter used when the source and observation points are at finite distances from the wedge. The variable $n$ is a measure of the interior angle of the wedge and may take on any value between 0 and 2 . A value of $n=2$ indicates an infinitely thin half-plane and $n=1.5$ is a ninety degree wedge such as found in building corners. The spatial spread- 


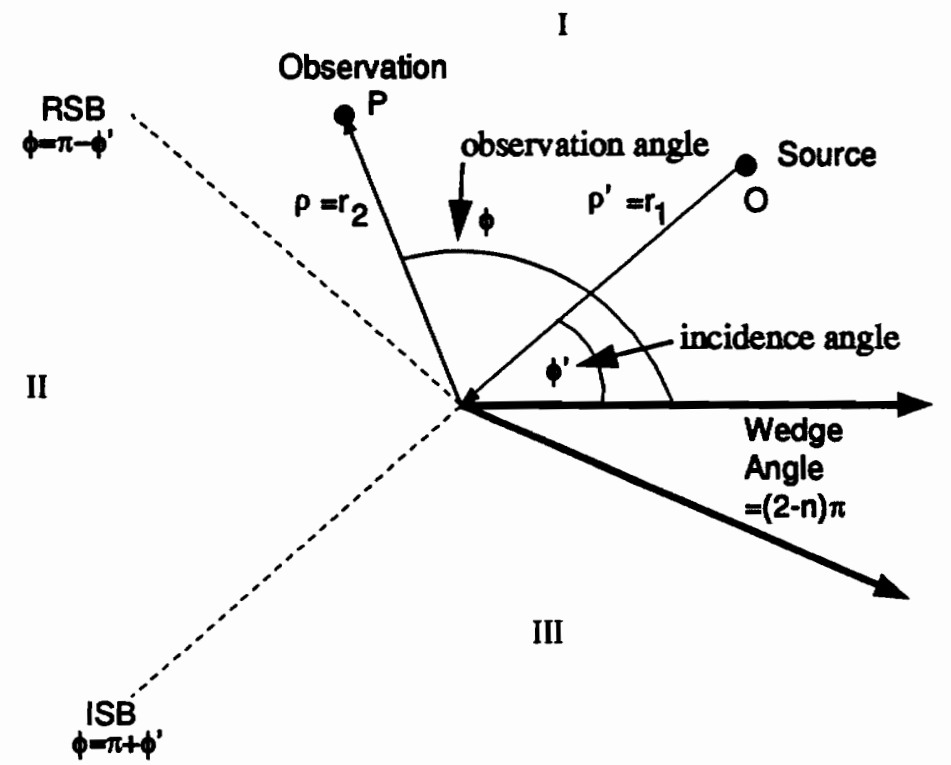

FIGURE 4.3-2. Wedge geometry for a perfectly conducting wedge. The source is a distance $\rho '=r_{1}$ from the tip of the wedge, and the observation point is at a distance $\rho=r_{2}$ from the tip of the wedge.

ing factor $A\left(\rho^{\prime}, \rho\right)$ in equation (4.3-1) for the diffracted ray is defined in equation (4.3-2) [Ba189].

$$
A\left(\rho^{\prime}, \rho\right)=\left\{\begin{array}{l}
\frac{1}{\sqrt{\rho}} \text { for cylindrical or plane wave incidence } \\
\sqrt{\frac{\rho^{\prime}}{\rho\left(\rho+\rho^{\prime}\right)}} \text { for spherical wave incidence }
\end{array}\right.
$$

Cylindrical wave incidence is useful for determining the scattered (diffracted) fields when the scattering body is infinite in one dimension and varies only in two dimensions. Since the object only varies in two dimensions, the fields only vary in two dimensions as well. Three-dimensional or spherical wave incidence must be used when the source is a point source and the scattering object varies in all three dimensions.

The diffraction coefficient is determined so that the geometrical theory of diffraction approximation is the same as the asymptotic form of the exact solution for the infinite wedge. The GTD diffraction coefficients developed by Keller are described in equations (4.3-3) and (4.3-4) [Kel57]. 


$$
\begin{aligned}
D_{s}\left(\rho, \phi, \phi^{\prime}, n\right)= & \frac{e^{-j \frac{\pi}{4}} \frac{1}{n} \sin \left(\frac{\pi}{n}\right)}{\sqrt{2 \pi k}} \times \\
& {\left[\frac{1}{\cos \left(\frac{\pi}{n}\right)-\cos \left(\frac{\phi-\phi^{\prime}}{n}\right)}-\frac{1}{\cos \left(\frac{\pi}{n}\right)-\cos \left(\frac{\phi^{\prime} \phi^{\prime}}{n}\right)}\right] } \\
D_{h}\left(\rho, \phi, \phi^{\prime}, n\right)= & \frac{e^{-j \frac{\pi}{4} \frac{1}{n} \sin \left(\frac{\pi}{n}\right)}}{\sqrt{2 \pi k} \times} \\
& {\left[\frac{1}{\cos \left(\frac{\pi}{n}\right)-\cos \left(\frac{\phi-\phi^{\prime}}{n}\right)}+\frac{1}{\cos \left(\frac{\pi}{n}\right)-\cos \left(\frac{\phi+\phi^{\prime}}{n}\right)}\right] }
\end{aligned}
$$

for soft (TE) and hard (TM) polarization. The terms hard and soft are derived from scalar acoustic scattering. For soft polarization, the electric field is zero on the boundary. This is the Dirichlet boundary condition and occurs when the polarization is TE to the scattering surface. For hard polarization, the normal derivative of the electric field (this is the magnetic field) on the surface is zero. This is the Neumann boundary condition that occurs when the polarization is TM with respect to the scattering surface. The Keller diffraction coefficients given in equations (4.3-3) and (4.3-4) yield the correct high frequency asymptotic fields everywhere except near the reflection shadow boundary and the incident shadow boundary as shown in Figure 4.3-6. The units of the diffraction coefficients $D_{s}$ and $D_{h}$ are square root of meters. The diffraction coefficients are determined by an approximation of the exact solution. The accuracy of the approximation depends on the angle between the observation angle and the shadow boundary. When the observation approaches the shadow boundary, the approximation is less accurate and the diffraction coefficients become singular. In order to determine the fields near these boundaries, a Uniform Geometrical Theory of Diffraction (UTD) was developed by Kouyoumjian and Pathak [Kou74]. The uniform diffraction coefficients are given as 


$$
\begin{aligned}
& D_{s}\left(L, \phi, \phi^{\prime}, n\right)=D^{i}\left(L, \phi-\phi^{\prime}, n\right)-D^{r}\left(L, \phi+\phi^{\prime}, n\right) \\
& D_{h}\left(L, \phi, \phi^{\prime}, n\right)=D^{i}\left(L, \phi-\phi^{\prime}, n\right)+D^{r}\left(L, \phi+\phi^{\prime}, n\right)
\end{aligned}
$$

where

$$
\begin{aligned}
& D^{i}\left(L, \phi-\phi^{\prime}, n\right)=-\frac{e^{-j \frac{\pi}{4}}}{2 n \sqrt{2 \pi k}} \times \\
& \quad\left\{\cot \left(\frac{\pi+\left(\phi-\phi^{\prime}\right)}{2 n}\right) F\left[k L g^{+}\left(\phi-\phi^{\prime}\right)\right]+\cot \left(\frac{\pi-\left(\phi-\phi^{\prime}\right)}{2 n}\right) F\left[k L g^{-}\left(\phi-\phi^{\prime}\right)\right]\right\} \\
& D^{r}\left(L, \phi+\phi^{\prime}, n\right)=-\frac{e^{-j \frac{\pi}{4}}}{2 n \sqrt{2 \pi k}} \times \\
& \quad\left\{\cot \left(\frac{\pi+\left(\phi+\phi^{\prime}\right)}{2 n}\right) F\left[k L g^{+}\left(\phi+\phi^{\prime}\right)\right]+\cot \left(\frac{\pi-\left(\phi+\phi^{\prime}\right)}{2 n}\right) F\left[k L g^{-}\left(\phi+\phi^{\prime}\right)\right]\right\}
\end{aligned}
$$

The distance parameter $L$ is given as $\frac{\rho \rho^{\prime}}{\rho+\rho^{\prime}}$ for cylindrical or spherical waves, and $k$ is the free space wavenumber. The functions $\mathrm{g}^{+}$and $\mathrm{g}^{-}$are representative of the angular separation between the observation point and the incident or reflection shadow boundary [Kou74], [Bal89].

$$
\begin{aligned}
& g^{+}=1+\cos \left[\left(\phi \mp \phi^{\prime}\right)-2 n \pi N^{+}\right] \\
& g^{-}=1+\cos \left[\left(\phi \mp \phi^{\prime}\right)-2 n \pi N^{-}\right]
\end{aligned}
$$

where $\mathrm{N}^{+}$or $N$ is the integer which most closely satisfies the equation

$$
\begin{aligned}
& 2 n \pi N^{+}-\left(\phi \mp \phi^{\prime}\right)=+\pi \text { for } \mathrm{g}^{+} \\
& 2 n \pi N^{-}-\left(\phi \mp \phi^{\prime}\right)=-\pi \text { for } \mathrm{g}^{-}
\end{aligned}
$$


This is described in detail in [Kou74]. The transition function $F(X)$ is defined as

$$
F(X)=2 j \sqrt{X} e^{j X} \int_{\sqrt{X}}^{\infty} e^{-j \tau^{2}} d \tau
$$

where $X$ is a dummy variable for the argument of the transition function in equations (4.37) and (4.3-8).

The uniform diffraction coefficients given in equations (4.3-5) through (4.3-8) do not possess singularities at the incident and reflection shadow boundaries. The singularities in the Keller diffraction coefficients are overcome essentially by the multiplication of the diffraction coefficients by a Fresnel integral as given in equation (4.3-13). At the shadow boundary, the Fresnel integral is zero in such a way that the product of the integral and the Keller diffraction coefficient remains finite, and away from the shadow boundaries, the Fresnel integral is unity. Once the diffraction coefficients are found, equation (4.3-1) can be used to solve for the diffracted field. The fields determined by uniform diffraction theory contain the correct discontinuities to completely cancel the discontinuities found in the geometrical optics solution at the shadow boundaries. Hence, the total field is continuous.

As an example of how the uniform diffraction coefficients compensate for the discontinuities in the geometrical optics field, consider the perfectly conducting wedge shown in Figure 4.3-1 and Figure 4.3-2. Assume a plane wave is incident on an infinite half-plane $(n=2)$ at an angle of $\phi^{\prime}=30$ degrees. The incident diffracted, reflected diffracted, and geometrical optics fields at an observation distance of one wavelength from the tip of the wedge are shown in Figure 4.3-3. The total field is given as the phasor sum of the geometrical optics, incident diffracted and reflected diffracted fields. Notice that the discontinuities present in the geometrical optics field at the reflection shadow boundary (RSB) and incident shadow boundary (ISB) are compensated by the discontinuities in the diffracted fields to give a continuous total field distribution. 


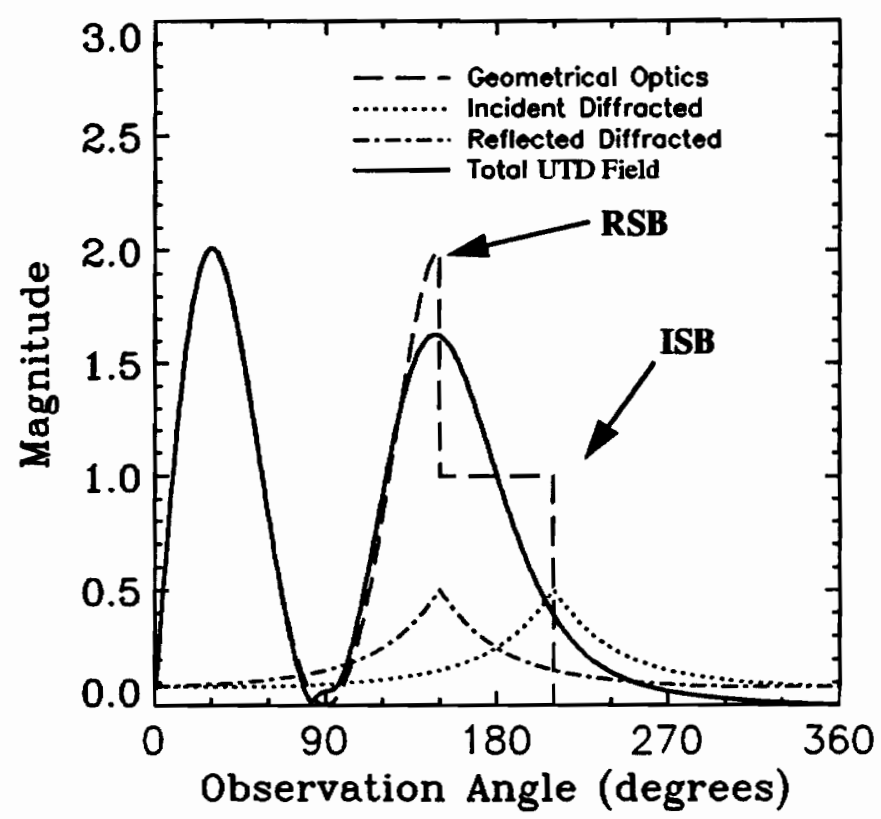

FIGURE 4.3-3. Field distribution of various components of a soft polarized plane wave incident normally on a conducting half-plane (from [Bal89]). The geometrical optics field, the incident diffracted field, and the reflected diffracted field are shown along with the total field which is the phasor sum of the geometrical optics, incident diffracted, and reflected diffracted fields. The observation angle is the angle of the receiver as measured from the face of the wedge as indicated in Figure 4.3-2.

Figure 4.3-5 shows the different field components for scattering of a spherical wave by an infinite length flat strip computed using the uniform theory of diffraction. The strip is shown in Figure 4.3-4. The diffracted field from each edge of the strip has a discontinuity to compensate for the shadow boundaries in the reflected geometrical optics field. The continuous field represented by the dark line in Figure 4.3-5 is the total scattered field as a function of scattering angle in the forward direction. Figure 4.3-6 shows the solution for the same case as in Figure 4.3-5 for computations with geometrical optics, geometrical theory of diffraction, and uniform theory of diffraction. Notice that the Keller GTD diffraction coefficients are singular at the shadow boundaries and do not give the correct solution between about twenty and forty degrees. The UTD diffraction coefficients give a continuous field and should be used to determine the scattering. 


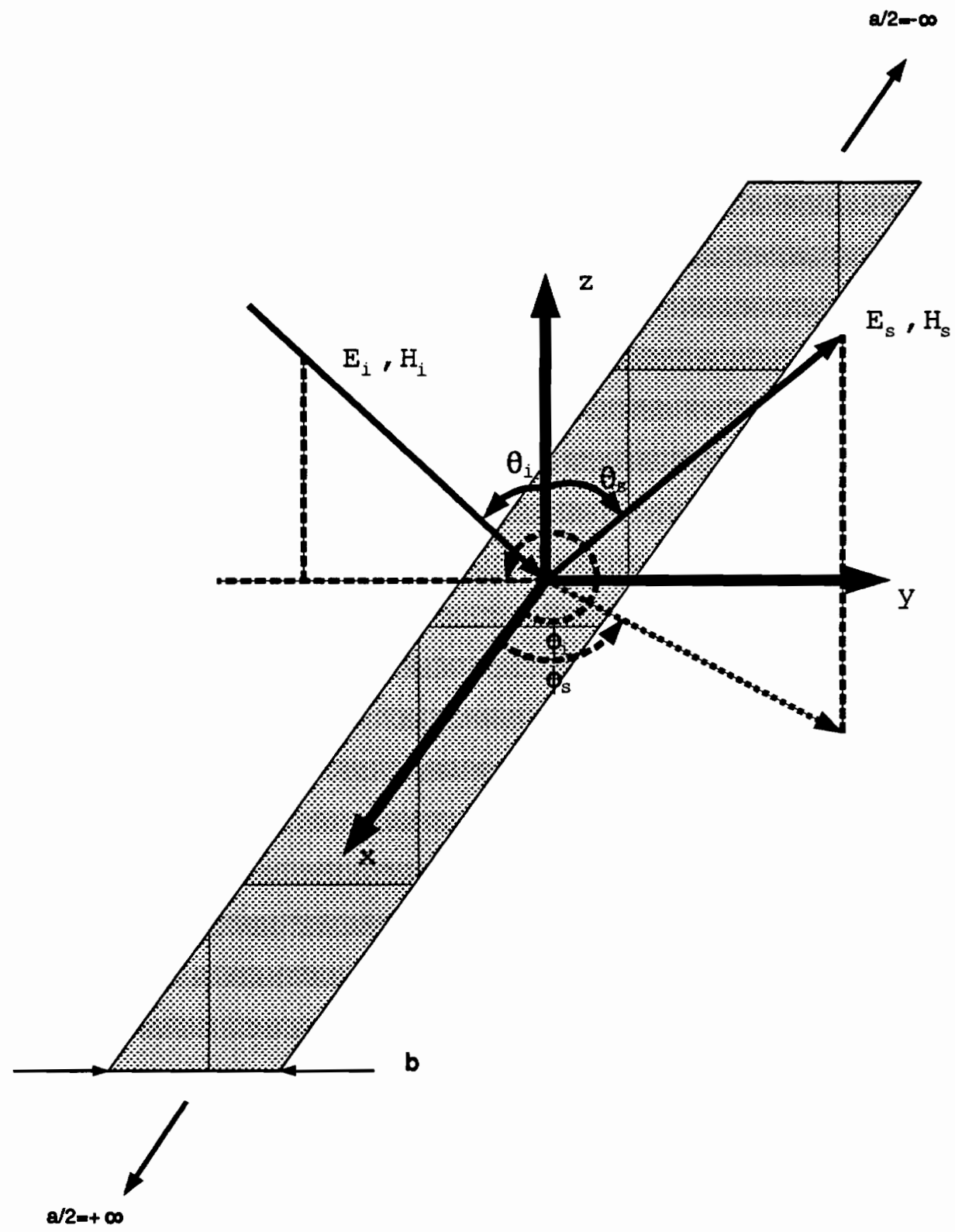

FIGURE 4.3-4. Geometry for scattering from an infinitely long strip of finite width $b$. 


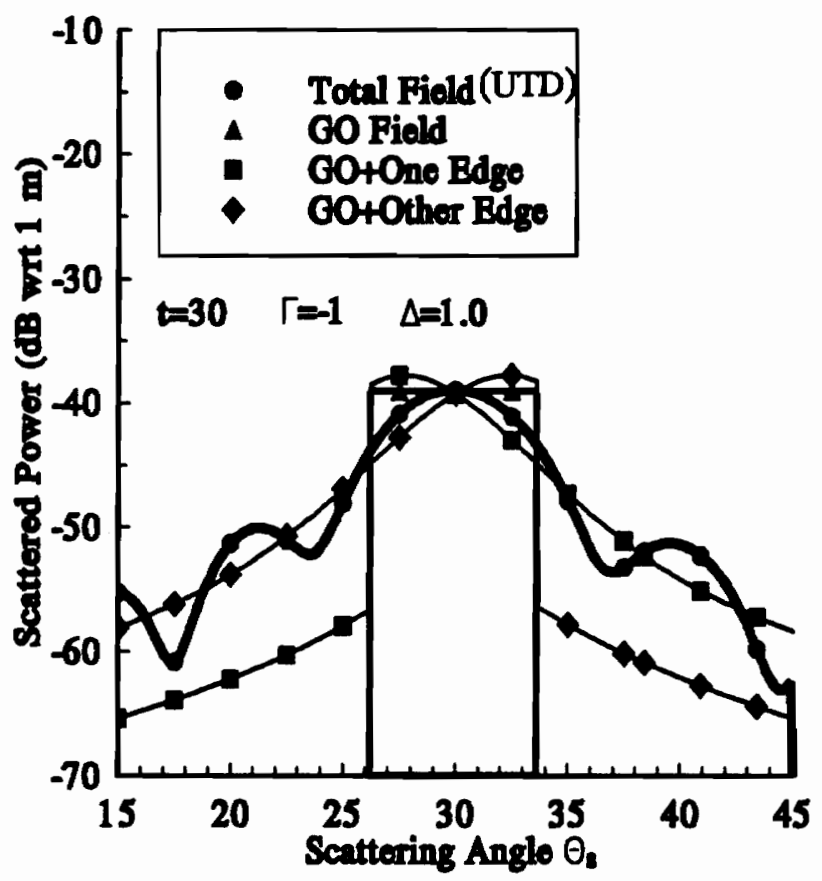

FIGURE 4.3-5. Individual UTD field components scattered from an infinitely long finite width strip shown in Figure 4.3-4.

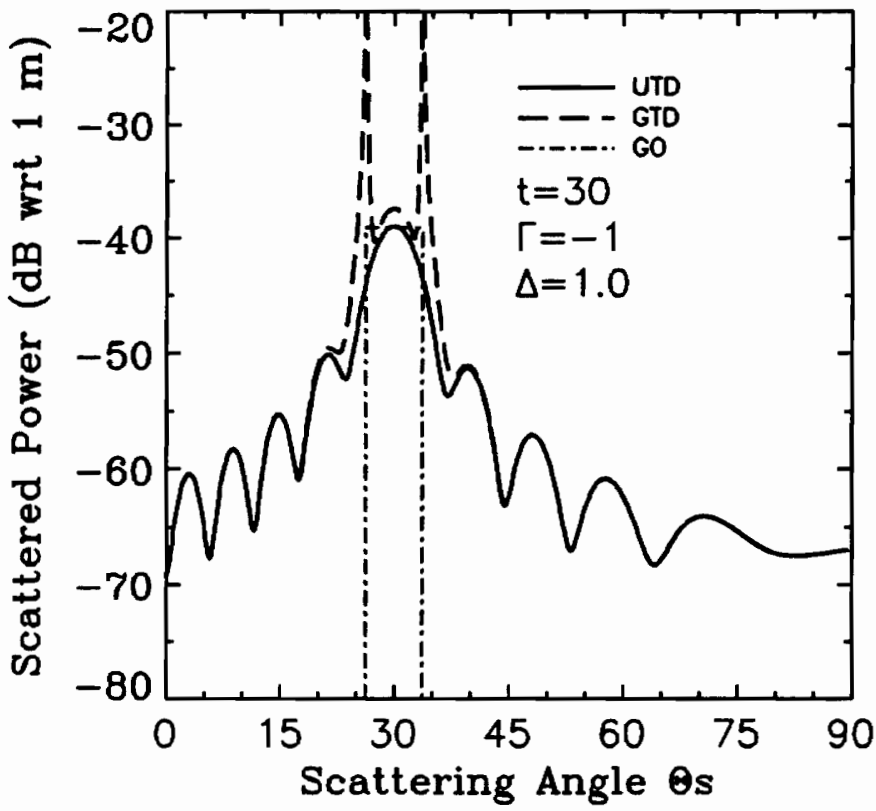

FIGURE 4.3-6. GO, GTD, and UTD diffraction solutions for scattering from a finite width strip. Notice that the Keller GTD solution is singular at the shadow boundaries. 


\subsubsection{Dielectric Bodies}

The diffraction coefficients in Section 4.3.1 Perfectly Conducting Surface were developed from the canonical scattering solution for perfectly conducting wedges. The canonical problem of scattering by a dielectric wedge is as yet unsolved. Hence, diffraction coefficients can not be derived directly from the solution. Instead, the diffraction coefficients are modified so that the continuity of the total field at the shadow boundaries is maintained. Each diffraction coefficient is made up of an incident diffracted term and a reflected diffracted term. These individual components of the diffraction coefficients for a perfectly conducting wedge are modified so that the total diffraction coefficient for a dielectric edge correctly compensates for the field discontinuities at the shadow boundaries in the geometrical optics solution. The diffraction coefficients become [Bur83]:

$$
D\left(\phi, \phi^{\prime}\right)=\left[(1-T) D_{i}\left(\phi-\phi^{\prime}\right)+\Gamma D_{r}\left(\phi+\phi^{\prime}\right)\right]
$$

where $\Gamma$ and $T$ are the reflection and transmission coefficients of the surface along the shadow boundaries. The modified uniform geometrical theory of diffraction solution is continuous and produces the same shape of scattering pattern as the perfectly conducting wedge solution. Although the shape of the scattering pattern is the same, the scattered power is affected by the reflection coefficients. This method for computing the modified diffraction coefficients has been used to model path loss over hills or ridges [Lue84a], [Lue84b].

\subsubsection{Application of Uniform GTD to a Perfectly Conducting Flat Rectangular Plate}

Geometrical optics considers reflection from infinite planar surfaces. In reality, surfaces are finite and edge effects can alter the scattering pattern. Here, two different models are used to determine the scattering by a rectangular flat plate finite in two dimensions. The regions of the validity of the two models differ. One model is used in the farfield, and the other is used when the receiver is in the near-field of the scatterer. The finite strip model considers the scattering from a strip finite in one dimension and infinite in the other. Since there is no variation in one dimension, the two-dimensional scattering solution is computed for cylindrical wave incidence and scattering. Once the two-dimensional 
fields are computed, the ends of the strip are eliminated so that the strip becomes finite in two dimensions. The cylindrical fields can be converted to three-dimensional spherically spreading fields when the strip is in the far-field of the source (local plane wave) and the observation point is in the far-field of the strip. A far-field conversion factor that incorporates the finite length of the strip in the transverse direction is used to convert the fields from two dimensions to three [Sie58]. Hence, the finite dimensions of the plate in both dimensions are considered in the far-field solution. The infinite strip model assumes that the scattering from the flat plate is the same as the scattering from an infinite length strip when the incident and scattered waves are spherical. The finite length of the strip in the transverse direction is ignored. No restriction is placed on the distance of the scatterer from the source. The distance that denotes the transition point for which model is used is the location of the first Fresnel zone at $2 L^{2} / \lambda$ where $L$ is the largest dimension of the scatterer. The finite strip model is used for distances greater than the location of the first Fresnel zone, and the infinite strip model is used for observation distances smaller than the first Fresnel zone distance. These models are developed to show how the uniform geometrical theory of diffraction can be used to determine the scattering and diffraction of radio waves is implemented for objects in buildings.

\section{Finite Strip Model}

Consider a cylindrically spreading wave incident upon a finite width perfectly conducting strip. The geometry is shown in Figure 4.3-4 where the variable $a$ is first considered to extend to infinity. Once the solution in two-dimensions is computed, the length $a$ is reduced to a finite value to convert the fields to three dimensions. The cylindrical scattered fields consist of the geometrical optics reflected field from the surface of the strip and a diffraction contribution from each of the two edges. Each edge is modeled as an infinite half plane. For a wave polarized in the $+x$ direction, the polarization is TE with respect to the two edges. Multiple diffraction is not considered.

The geometrical optics scattered field is 


$$
\vec{E}_{G O}=-E_{0} \hat{x} \frac{e^{-j k\left(r_{1}+r_{2}\right)}}{\sqrt{r_{1}+r_{2}}}
$$

in the specular directions only, and each diffracted field is

$$
\vec{E}_{D}=E_{0} \hat{x} \frac{e^{-j k\left(r_{1}+r_{2}\right)} D_{s}\left(L, \phi, \phi^{\prime}, n=2\right)}{\sqrt{r_{1} r_{2}}}
$$

where the distances $r_{1}$ and $r_{2}$ are the distances of the transmitter and receiver to the specular reflection point for the $\mathrm{GO}$ field and the distances to the edge diffraction point for the diffracted field. The variables $\phi$ and $\phi$ ' are the observation and incidence angle. Figure 4.3-7 shows the assumed geometry for one diffraction edge. The diffracted field exists everywhere, and the $G O$ field is zero when no specular point exists on the surface. A specular point exists on the surface if the receiver falls within the specular directions as indicated in Figure 4.3-8. The specular point is the intersection of line $A D$ and a straight line drawn between the receiver and the image source. The specular point lies on the surface if the intersection point is between points $B$ and $C$.

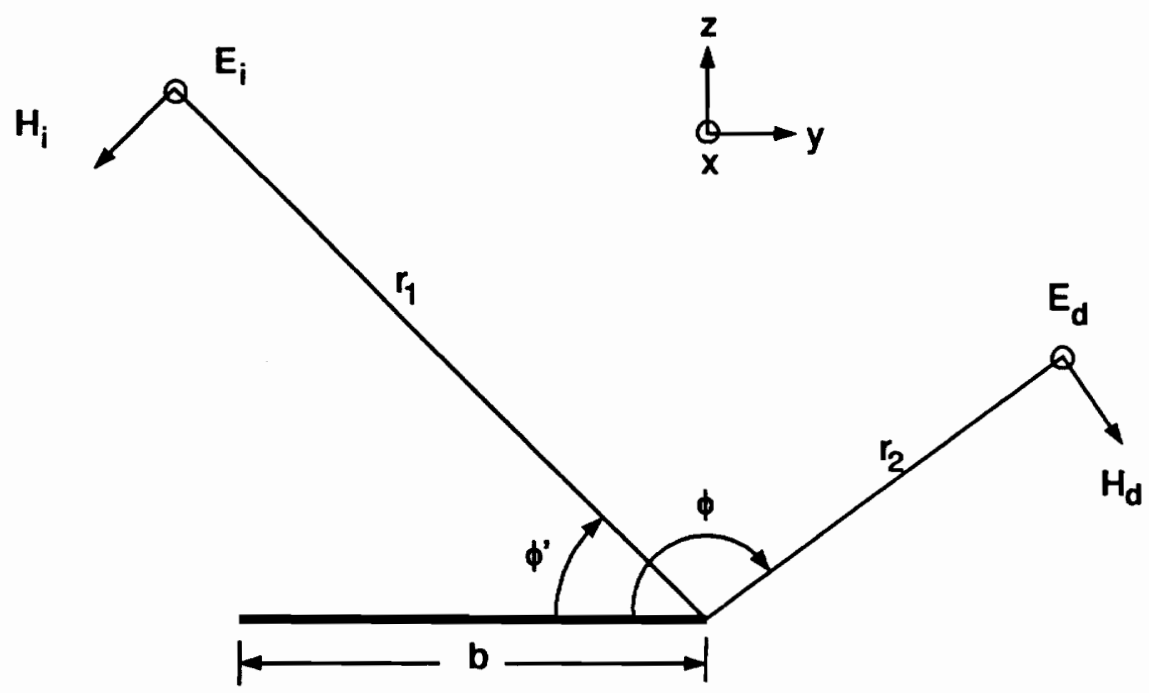

FIGURE 4.3-7. Two-dimensional view of diffraction geometry for scattering from an infinite length strip of finite width $b$. 


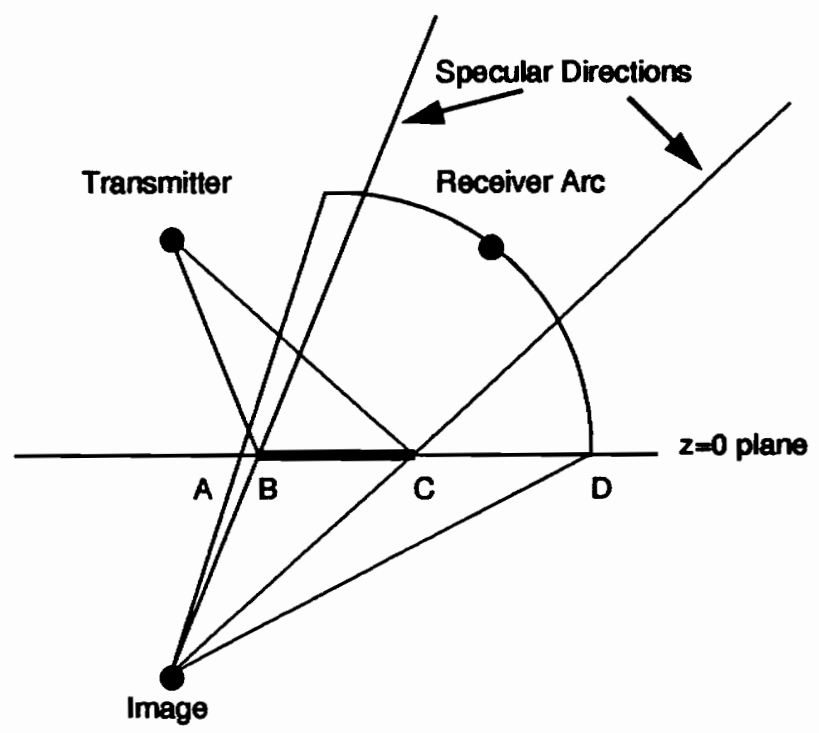

FIGURE 4.3-8. Transmitter and image source for scattering from a flat plate. The specular point is the intersection of line $\mathrm{AD}$ and a straight line drawn between the receiver and the image source. The specular point lies on the surface if the intersection point is between points $B$ and $C$.

The two-dimensional scattered field is

$$
\vec{E}_{S}=\vec{E}_{G O}+\vec{E}_{D 1}+\vec{E}_{D 2}
$$

In the far-field, the two-dimensional scattered fields can be converted to three-dimensional fields by multiplying by the following conversion factor that accounts for the finite length of the strip which is denoted by the variable $a$ [Sie58]:

$$
\frac{a e^{-j \frac{\pi}{4}}}{\sqrt{\lambda r_{2}}}
$$

This conversion factor arises from an integration over the length of the edge and effectively accounts for the finite length of the strip in the $\hat{x}$ direction. However, this conversion factor is derived from far-field approximations and is not valid when the observation point is closer than the first Fresnel zone distance [Sie58]. In addition, the scatterer must be in the far-field of the source such that the incident waves may be considered locally planar. Diffraction effects from the two edges that are perpendicular to the electric 
field vector will be cross-polarized, and will cancel each other for scattering in the principle plane $\left(\phi_{\mathrm{i}}=270^{\circ}, \phi_{\mathrm{s}}=90^{\circ}\right)$. Comparison of measurements and calculations of the radar cross section of rectangular flat plates in [Ros66] show that these edges can be ignored for lengths greater than about two wavelengths.

\section{Infinite Strip Model}

The far-field conversion factor cannot be used when the observation point is close to the scatterer. As a model for the scattering from a perfectly conducting flat plate, the scattering is assumed to be the same as that from a perfectly conducting infinite strip with the same width as the plate. The finite length of the plate in the transverse direction is ignored, and spherical wave incidence and scattering are assumed. The scattered geometrical optics field in three dimensions is assumed to be the far-field GO field

$$
\vec{E}_{G O}=-E_{0} \hat{x} \frac{e^{-j k\left(r_{1}+r_{2}\right)}}{r_{1}+r_{2}}
$$

when a specular point exists on the surface, and each diffracted field is

$$
\vec{E}_{D}=E_{0} \hat{x} \frac{e^{-j k\left(r_{1}+r_{2}\right)} D_{s}\left(L, \phi, \phi^{\prime}, n=2\right)}{\sqrt{r_{1} r_{2}\left(r_{1}+r_{2}\right)}}
$$

The total scattered field is

$$
\vec{E}_{S}=\vec{E}_{G O}+\vec{E}_{D 1}+\vec{E}_{D 2}
$$

In Section 4.6 Computed Scattering and Diffraction Patterns, the computations of the scattering from a finite rectangular flat plate for both near-field and far-field conditions is presented. It is shown that both the near-field and the far-field model compare well with the physical optics solution in the specular direction when each model is used in the appropriate region of validity. The physical optics solution in the specular direction is considered an accurate approximation to the exact scattering solution. In the far-field, the length of the plate in the transverse direction 'appears' small, and a far-field conversion factor in the finite strip model may be used to account for the finite length of the plate. 
When the observation is close to the scatterer, the length of the plate approaches an infinite strip and 'appears' much larger. Hence, the length of the plate is ignored and the infinite strip model is used.

\subsection{Physical Optics (PO)}

Scattering from finite size bodies can be determined via the vector potential method for radiation from a finite current source [Bal89]. This method determines the radiation (scattering) by integration of the surface currents over the extent of the body. First the vector potentials are found from the surface currents, and then the scattered fields are found from the vector potentials. Since the exact currents on the surface of the scatterer are often unknown in a scattering problem, physical optics is used to estimate the surface currents. Physical optics is a high frequency approximation for the currents on the surface of an arbitrary scatterer such as shown in Figure 4.1-1 through Figure 4.1-4. Physical optics assumes that the surface currents on the body are determined by the fields at the surface of the scattering body in the lit region shown in Figure 4.1-3. The surface currents are assumed to be zero in the shadow region. This approximation improves as the size of the scatterer relative to a wavelength increases. Once the surface currents are assumed, the problem to be solved becomes that of a radiator in free space. Hence, the vector potential method can be used to determine the scattered fields. Essentially, an integration (spatial Fourier Transform) over the surface of the scatterer is performed to determine the fields. The fields at the surface of the body used to determine the physical optics surface current are the geometrical optics $(\mathrm{GO})$ fields at the surface. The geometrical optics fields were described in Section 4.2 Geometrical Optics [Bor65], [Des72], [Col85], [Van85]. This implies that, at each illuminated point on the scatterer, the scattering takes place as if there were an infinite tangent plane at that point [Stu81]. The local plane wave Fresnel reflection coefficients are used to determine the GO fields at the surface. Physical optics is considered to be more general than geometrical optics since in the high-frequency limit, the physical optics solution contains the wave-like nature of the field about the specular direction. Physical optics can be expected to provide an accurate representation of the scattered field when the postulated physical optics current is reasonably close to the true current dis- 
tribution [Stu81]. Physical optics is most accurate in the specular scattering direction, and fails when the scattering direction is far from the specular direction because the effects of edges are ignored [Ros66], [Kno85]. When scattering from finite flat plates is considered, edge effects can become significant since the true surface current and the surface current postulated by physical optics differ. Other techniques that incorporate the edge effects such as the geometrical theory of diffraction should be used. A physical optics model has been used to model the scattering from buildings in an urban mobile radio environment [Noe86].

Consider a flat planar surface of area $a b$ as shown in Figure 4.4-1. A detailed

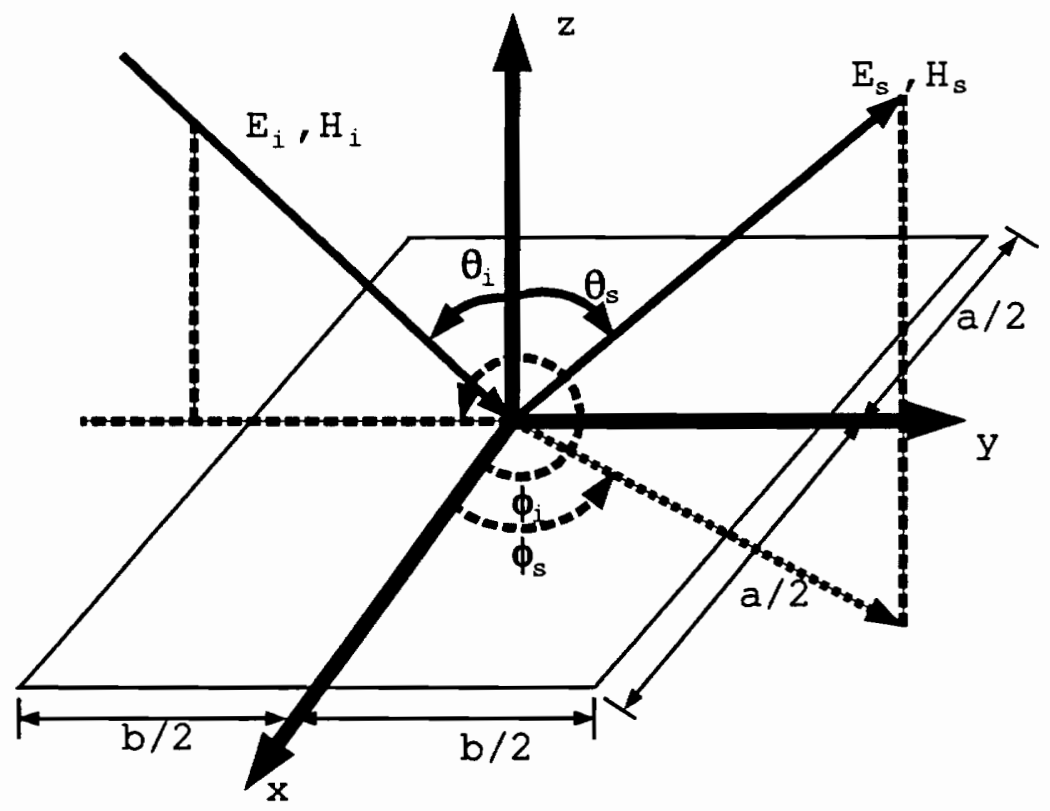

FIGURE 4.4-1. Geometry for scattering by a perfectly conducting flat plate with area $a b$. An electromagnetic wave is incident at an angle $\theta_{i}$ from the surface normal, and $\phi_{\mathrm{i}}$ from the $\mathrm{x}$-axis as indicated in the figure.

development of the scattering solution based on physical optics is given in Appendix B. An electromagnetic wave is incident at the angles $\theta_{i}, \phi_{i}$ with respect to the center of the surface. This wave is TE with respect to the propagation direction. The polarization is shown in Figure 4.4-2. For a dielectric and TE polarization, the induced electric and mag- 
Perpendicular (TE) Polarization

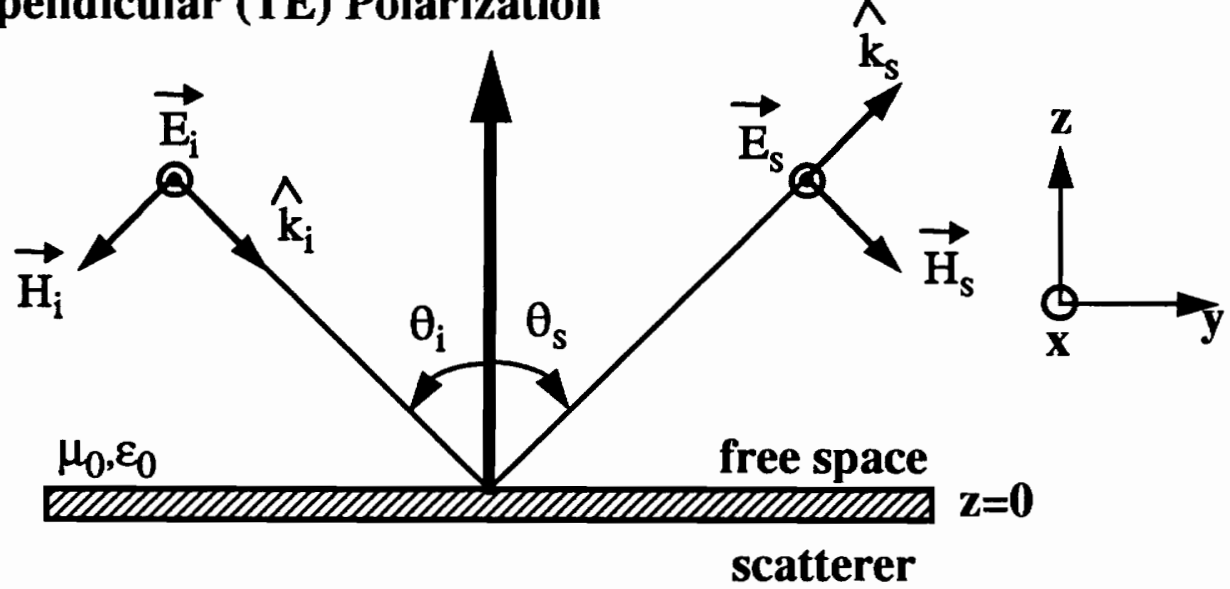

FIGURE 4.4-2. Incident and scattered field polarization of a perpendicularly (TE) polarized plane wave incident upon a finite flat plate scatterer $\left(\phi_{i}=270, \phi_{s}=90\right)$.

netic surface currents are proportional to the total tangential fields on the surface. The total tangential fields are the sum of the incident and reflected fields on the surface of the scatterer. Hence, the surface currents are

$$
\begin{gathered}
\vec{J}_{s} \cong \frac{E_{0}}{\eta r_{1}}\left(1-\Gamma_{\perp}\left(\vec{r}^{\prime}\right)\right)\left[\cos \theta_{i} \cos \phi_{i} \hat{y}-\cos \theta_{i} \sin \phi_{i} \hat{x}\right] e^{-j k r_{1}} \\
\vec{M}_{s} \cong \frac{E_{0}}{r_{1}}\left(1+\Gamma_{\perp}\left(\vec{r}^{\prime}\right)\right)\left[\sin \phi_{i} \hat{y}+\cos \phi_{i} \hat{x}\right] e^{-j k r_{1}}
\end{gathered}
$$

For a perfect electric conductor (PEC), $\Gamma_{\perp}=-1$ and the magnetic surface current is zero. These currents are exact for an infinite plane, but approximate for the scatterer in Figure 4.4-1, due to the neglect of the edges. These currents are the physical optics approximations for the true currents.

The scattered fields are determined by the radiation from the currents on the surface of the plate. The vector potentials are

$$
\vec{A}(\vec{r})=\frac{\mu}{4 \pi} \iint_{S^{\prime}} \vec{J}_{s}\left(\vec{r}^{\prime}\right) \frac{e^{-j k R}}{R} d s^{\prime}
$$




$$
\vec{F}(\vec{r})=\frac{\varepsilon}{4 \pi} \iint_{S^{\prime}} \vec{M}_{s}\left(\vec{r}^{\prime}\right) \frac{e^{-j k R}}{R} d s^{\prime}
$$

where $R=|\vec{r}-\vec{r}|$ is the distance from a point on the surface to the observation point, and the integration is performed over the entire surface of the plate $S$ '. The total scattered electric field is the superposition of the electric field due to each of the vector potentials.

$$
E_{A_{x}}=-\frac{j \eta E_{0}}{4 \pi k} \iint_{S^{\prime}}\left\{G_{1} J_{x}+\left(x-x^{\prime}\right) G_{2} \times\left[\left(x-x^{\prime}\right) J_{x}+\left(y-y^{\prime}\right) J_{y}\right]\right\} e^{-j k R} d s^{\prime}
$$

where

$$
G_{1}=\frac{-1-j k R+k^{2} R^{2}}{R^{3}} \text { and } G_{2}=\frac{3+j 3 k R-k^{2} R^{2}}{R^{5}}
$$

The electric field due to the vector potential $\vec{F}$ is

$$
E_{F_{x}}=-\frac{1}{4 \pi} \iint_{S^{\prime}}\left(z-z^{\prime}\right) M_{y}\left(\frac{1+j k R}{R^{3}}\right) e^{-j k R} d s^{\prime}
$$

and the total electric field polarized in the $\hat{x}$ direction is $E_{T_{x}}=E_{A_{x}}+E_{F_{x}}$.

Equations (4.4-5) and (4.4-7) are the exact expressions for the scattered field due to a surface current and these equations are valid in the near field. The accuracy is determined by the accuracy of the physical optics assumption for the electric and magnetic currents on the surface of the plate in equations (4.4-1) and (4.4-2).

\subsection{Radar Cross Section}

\subsubsection{The Bistatic Radar Equation in Multipath Environments}

When the surface is sufficiently rough such that the energy is not carried in the specular direction, or the scattering in directions other than specular are required, the bistatic radar equation may be used to model the scattered energy. The radar cross-section of a target is a quantitative measure of the ratio of power density in the vector signal scat- 
tered in the direction of the receiver to the power density of the radio wave incident upon the target (scattering object) [Sko70]. The theoretical definition of RCS is taken as a limit as the distance $\mathbf{R}$ from the scatterer approaches infinity so that a hypothetical observation is made far enough away from the target that only $R^{-1}$ dependence appears in the scattered electric field. Near field terms that depend on $R^{-2}$ and $R^{-3}$ are not included in the radar cross section model. Note that this distance dependence is the distance dependence of the scattered (re-radiated) field relative to the field incident upon the scattering body, and is not the total field dependence with distance. In addition, radar cross-sections of scattering objects are functions of angle of incidence, angle of reflection, wave polarization, frequency, and orientation of the object. Figure 4.5-1 shows an application of the bistatic radar equation for detecting the presence of aircraft. Table 4.5-1 summarizes the variables used in the bistatic radar equation below.

TABLE 4.5-1: Table of variables used in the bistatic radar equation.

\begin{tabular}{|c|c|}
\hline Variable & Doscription [units] \\
\hline $\mathbf{P}_{\mathbf{R}}$ & Received power [dBm] \\
\hline $\mathbf{P}_{\mathbf{T}}$ & Transmitted power $[\mathrm{dBm}]$ \\
\hline $\mathrm{G}_{\mathrm{R}}$ & Receiver antenna gain [dBi] \\
\hline $\mathbf{G}_{\mathbf{T}}$ & Transmitter antenna gain [dBi] \\
\hline$\lambda$ & Carrier wavelength [meters] \\
\hline $\operatorname{RCS} \sigma_{b}$ & Radar cross-section [ $\mathrm{dBm}^{2}$ ] \\
\hline $\mathrm{D}_{\mathrm{T}}$ & Distance from transmitter to scattering object [meters] \\
\hline $\mathrm{D}_{\mathrm{R}}$ & Distance from receiver to scattering object [meters] \\
\hline d & T-R separation [meters] \\
\hline
\end{tabular}

$$
\begin{aligned}
& P_{R}[\mathrm{dBm}]=P_{T}[\mathrm{dBm}]+G_{T}[\mathrm{dBi}]+G_{R}[\mathrm{dBi}]+20 \times \log _{10}(\lambda) \\
& \quad+\mathrm{RCS}\left[\mathrm{dBm}^{2}\right]-30 \times \log _{10}(4 \pi)-20 \times \log _{10}\left(D_{T}\right)-20 \times \log _{10}\left(D_{R}\right)
\end{aligned}
$$

The bistatic radar equation describes the propagation of a wave that propagates in free space, is intercepted by a scattering object, and is then re-radiated in the direction of the receiver. The ratio of power intercepted and re-radiated is the radar cross-section of the scatterer. 


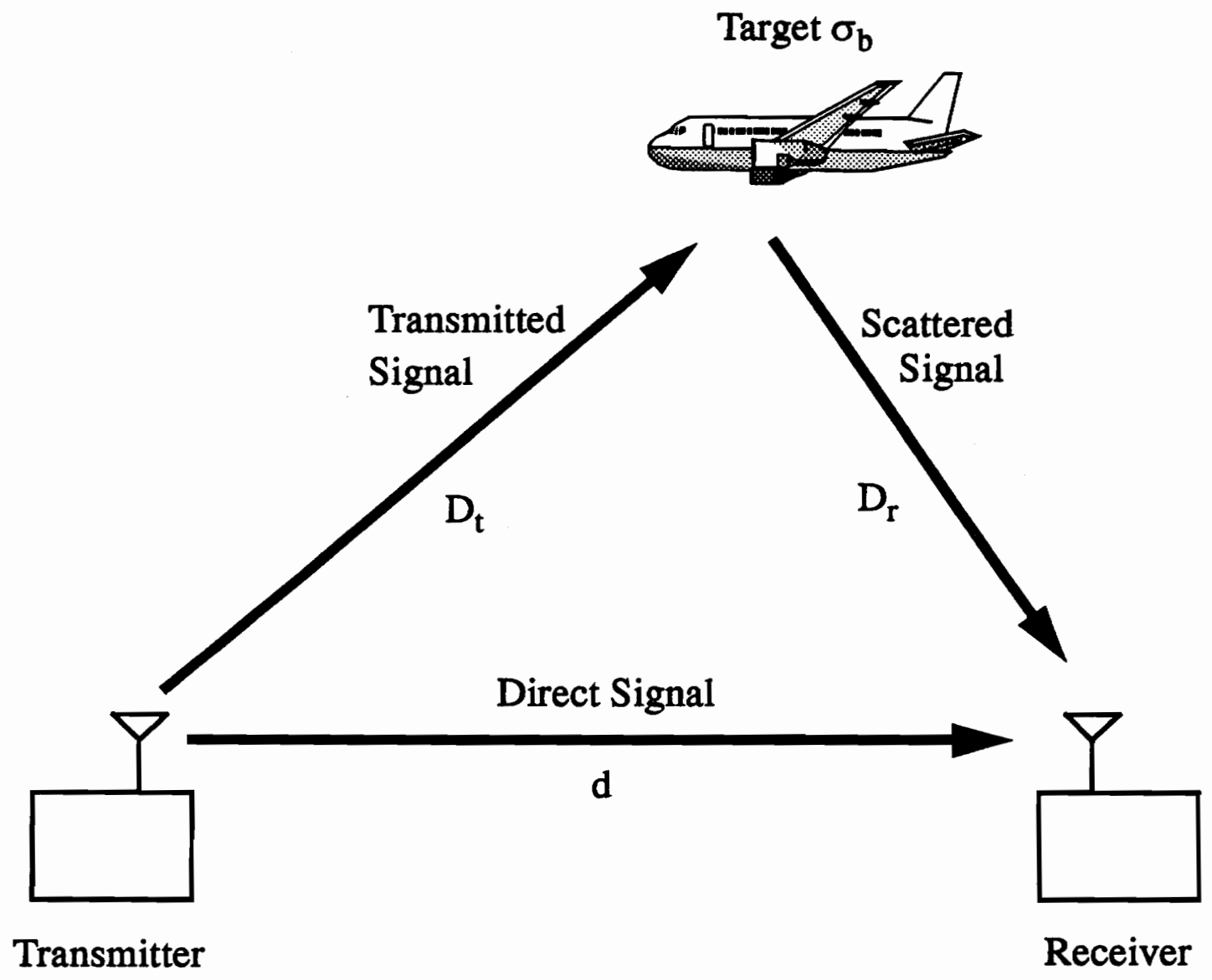

FIGURE 4.5-1. Bistatic radar used to detect the presence of aircraft (from [Sko80]).

Time domain multipath propagation measurements are, in essence, a bistatic radar measurement of the environment. Scattering objects are detected by the time delay of the individual multipath component. Thus, it seems reasonable that the impulse response of such a channel could be described in terms of a radar cross-section model. In most cases of practical concern, the RCS of a scattering object can vary by as much as 20-30 dB or more for small changes in frequency, angle of incidence, angle of reflection, wave polarization, and orientation of the object [Sko70]. The number of electromagnetic scattering problems that can be solved directly is small. 
In [van87], [Sei91b], and [Sei92c], a radar cross-section model was proposed to model the scattering of radio signals from buildings that are external to the transmitter and receiver. A radar cross-section (RCS) model is explained here to characterize the effects of surrounding buildings on propagation measurements where the transmitter and receiver are inside the same building. The radar cross-section model is a far-field model. Not only must the transmitter and receiver be in the far-field of each other, they must also be in the far-field of each scatterer. A common measure of when an object is in the far field is when $R>2 D^{2} / \lambda$, where $R$ is the distance from the object to the observation point, $D$ is the maximum dimension of a scatterer and $\lambda$ is the carrier wavelength [Sko70]. For a building with a maximum dimension of $10 \mathrm{~m}$, and a carrier frequency of $1900 \mathrm{MHz}$, the observer must be $1.3 \mathrm{~km}$ away from the building to be in the far-field. Hence, this model is not applicable for in-building work. Also, $R \gg D$ and $R \gg \lambda$ are required for the far-field approximation. Since only part of a building face may act as a scatterer, the $D$ used to determine the farfield region is not necessarily the largest spatial dimension, but may be the area illuminated by the antenna pattern.

The RCS model is a useful modeling technique for a first-order approximation for worst-case scattering, but accurate use of the model may be limited when buildings are close such that the far-field approximation is violated. Also, multiple reflections may occur, and the antenna pattern may influence the measurement.

For each multipath component recorded in each power delay profile in [Sei92c], the radar cross-section in $\mathrm{dBm}^{2}$ ( $\mathrm{dB}$ meters squared) required to explain the measurement was calculated. The receiver was located in a rural area on the outskirts of a large city and both the transmitter and receiver had line-of-sight to the downtown area of the city. Multipath components with large excess delays were observed and determined to be due to scattering from the city skyline. Since detailed building size and location information were not retained, the following assumptions were made to determine representative values from measured profiles.

1. All multipath components were assumed to be caused by a single scattering source, which may be due to either a single large object or group of objects in the environment. 
2. The scatterer was modeled by a RCS such that the received power was described by the bistatic radar equation given in equation (4.5-1) [Sko70].

3. The path lengths from the scatterer to the transmitter and receiver were assumed equal and could be determined from the time delay of the multipath component. While this is, in general, not true, it turns out to be a valid assumption when the transmitter and receiver are separated by multiple floors in the same building.

Application of the bistatic radar equation in [van87], [Sei91b], [Sei92c], shows that multipath with excess delays greater than a few microseconds can be explained with a physical, geometrical model. By computing RCS values with (4.5-1) for individual multipath components that were measured, a range of possible RCS values for surrounding buildings as a function of excess delay were determined in [Sei92c].

Figure 4.5-2 shows a scatter plot of the calculated RCS using equation (4.5-1) for all the received multipath components in three buildings in [Sei92c]. The received powers of individual multipath components were measured and the radar cross sections required to explain the measured power levels were determined. The letters NM, SR, and WC in

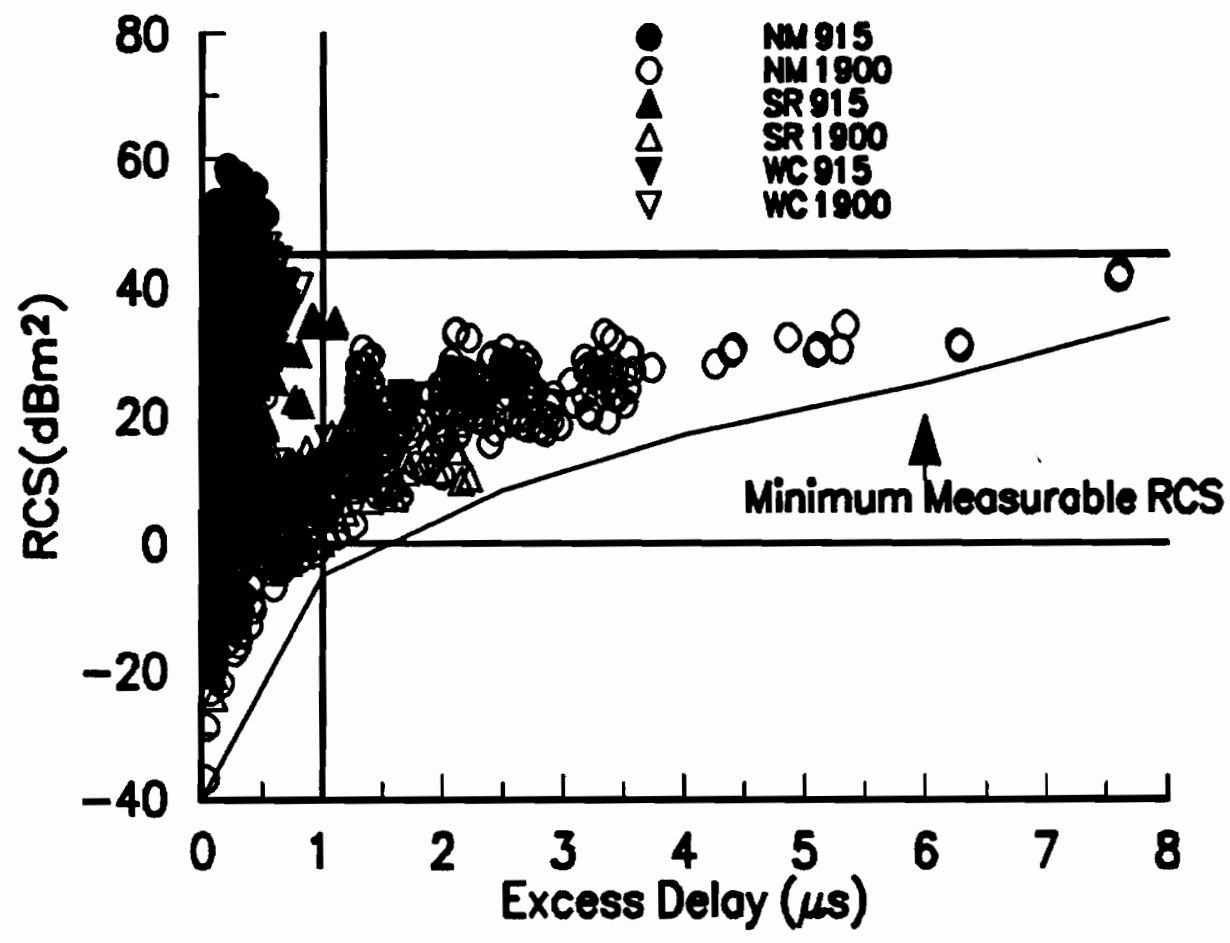

FIGURE 4.5-2. Scatter plot of observed radar cross-sections of buildings. 
the legend denote the different buildings, and the 915 and 1900 refers to the carrier frequency of the measurement in $\mathrm{MHz}$. The curved solid line indicates the approximate minimum measurable RCS for a given excess delay due to the dynamic range of the wide band receiver. Notice that for excess delays less than one microsecond, both small and large scatterers give rise to measured "RCS" values that range between -40 and +60 $\mathrm{dBm}^{2}$. For such small excess delays, the path lengths of the multipath components are not much longer than the distance between the transmitter and receiver. These scattering objects are most likely inside the building, and thus violate the far-field assumption. For such small excess delays, alternate techniques that require more information about the scatterer must be used for an accurate characterization. For multipath components with excess delays greater than one microsecond, it is more likely that the scattering objects are outside the building due to the increased propagation delay. These data seem to indicate that for multipath components with excess delays greater than two microseconds, a radar cross-section of between 0 and $+45 \mathrm{dBm}^{2}$ can be used to approximately characterize worst-case reflections from surrounding buildings. Although there are no measured data below the curved solid line, it is likely that other surrounding buildings produce radar cross-sections that cannot be detected by the measurement equipment.

The radar cross-section model can be used for worst-case system design for scattered signals from buildings outside the desired building when the transmitter and receiver are near windows with line-of-sight to the city skyline. Once further inside the building, the amplitudes of these scattered components must be modeled by alternate in-building techniques.

\subsubsection{Incoherent Power Summation}

The radar cross-section model described above is useful for modeling the scattering in non-specular directions when the receiver is in the far-field of large scattering objects. This model is not applicable to close-in receiver locations, and can only be used for worst-case system design. A heuristic model that subdivides a surface into multiple facets such that the receiver is in the far-field of each facet can be used to extend the region of validity for the radar cross-section model. 
In [Sch92], a heuristic model was implemented to supplement the geometrical optics fields predicted by ray tracing in microcellular environments. Scattering in nonspecular directions was accounted for by non-coherent summation of the powers scattered from each integration patch (surface facet) on the surface of the scatterer. The geometry for this model is given in Figure 4.5-3. This is equivalent to a 'miniature radar cross-section or mini-RCS' technique where the total scattered power is the sum of the powers scattered by each individual facet of the surface. The power scattered by each facet is determined by the bistatic radar equation for the facet given in equation (4.5-1). In this way, each facet acts as an independent scatterer with its own radar cross-section (RCS). The RCS of each facet is

$$
\sigma_{i}=\frac{4 \pi(\Delta x \Delta y)^{2}}{\lambda^{2}} \cos \theta \sigma_{r}
$$

where $\theta$ is the angle between the specular angle and the scattered wavevector, and $\sigma_{\mathrm{r}}$ is a loss factor applied since the surfaces modeled were not perfectly conducting. In addition to the 'scattered' power, the 'specular' power reflected by the surface is also received if the specular point lies within the boundaries of the surface. For scattering from rough building exteriors in [Sch92], a reflection coefficient of $-12 \mathrm{~dB}$ was used regardless of incidence angle, and a loss factor of $\sigma_{\mathrm{r}}=0.1$ was used. For the simulations here, the reflection coefficient was -1 for the perfectly conducting surface, but a loss factor of $\sigma_{\mathrm{r}}=0.1$ was used to modify the cross-section of each individual facet.

When used in a ray tracing propagation prediction program as in [Sch92], the individual surface facets are determined by the wavefronts represented by the individual rays that intercept the scattering object. The size of the wavefront at the scatterer is a function of the angular separation between rays and the distance that the ray path has traveled. This dependence occurs because the rays diverge from a point source. This implementation method would also be used for a physical optics scattering model. For the numerical computations presented here, the surface of the scatter was integrated by subdividing the surface into contiguous square patches. Both this method and the ray tracing implementation give the same calculations if the patch sizes are sufficiently small. Section 4.6.3 Scattering 


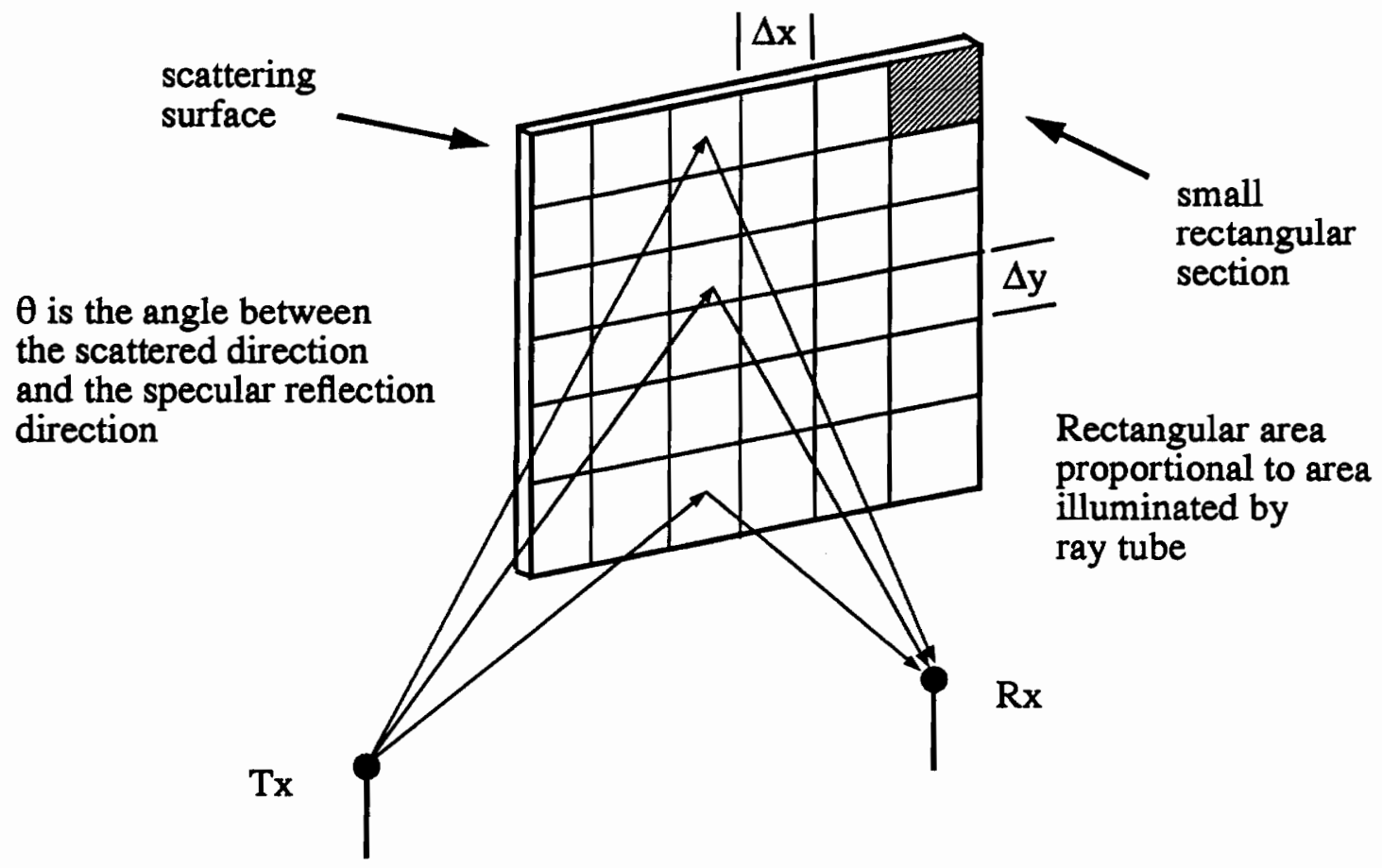

FIGURE 4.5-3. Geometry for non-coherent 'Mini-RCS' scattering model (from [Sch92].

Patterns as a Function of Different Parameters shows that each individual patch (or surface facet) should be smaller than $\lambda / 2$ by $\lambda / 2$ to give accurate results.

An alternative non-coherent model is given in equation (4.5-3) in which the scattered power is proportional to the observed surface area. Hence, the scattered power goes to zero as the observation angle approaches grazing.

$$
\sigma_{i}=\frac{4 \pi(\Delta x \Delta y)^{2}}{\lambda^{2}} \cos \theta_{1} \cos \theta_{2} \sigma_{r}
$$

The radar cross-section of each surface facet is proportional to the surface area 'seen' by both the transmitter and the receiver. The angles $\theta_{1}$ and $\theta_{2}$ are the angles that the incident ray and the reflected ray make with the outward pointing surface normal. The angles for the two non-coherent model in equations (4.5-2) and (4.5-3) are displayed in Figure 4.5-4. 


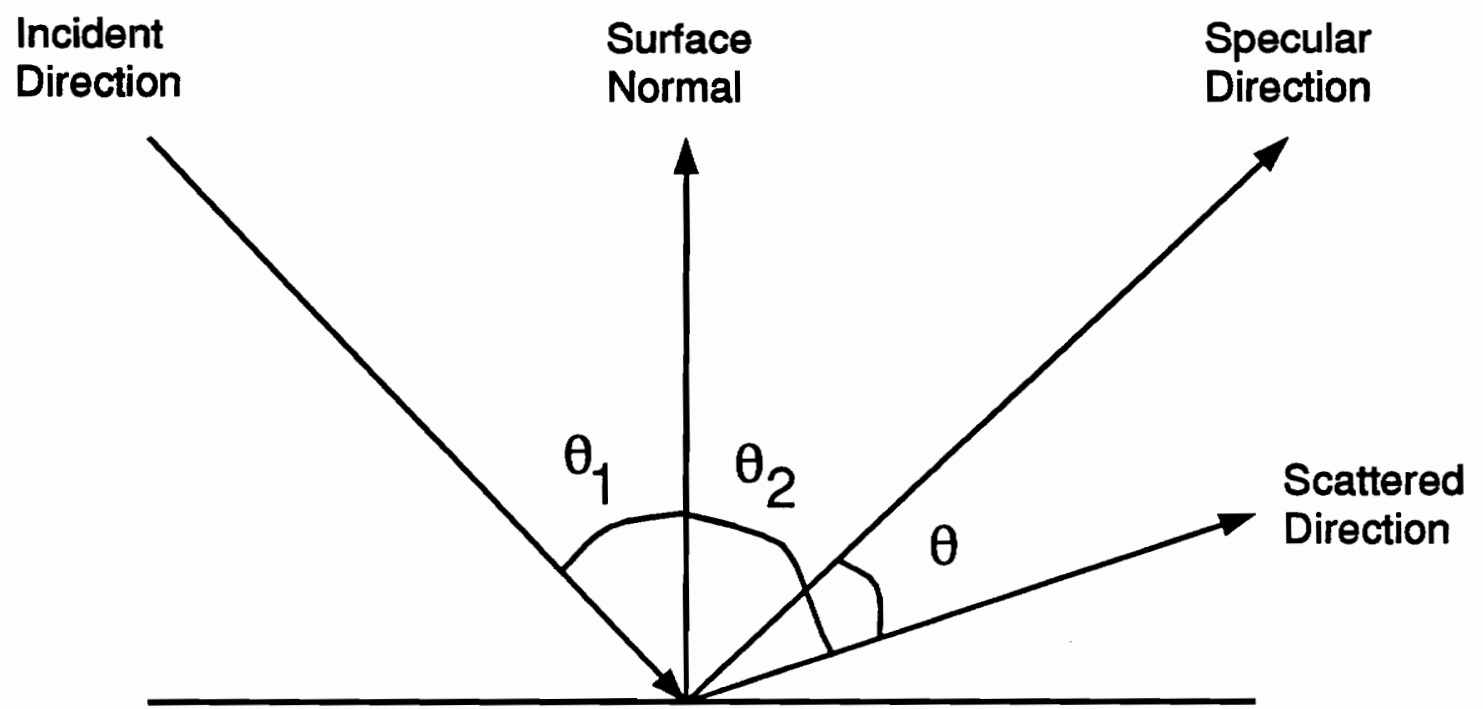

FIGURE 4.5-4. Incident and scattering angles for the two non-coherent scattering models given in equations (4.5-2) and (4.5-3).

\subsection{Computed Scattering and Diffraction Patterns}

\subsubsection{Numerical Comparison of Scattering and Diffraction Models}

To determine the regions of validity for each scattering and diffraction model, the scattering from a perfectly conducting ( $\Gamma=-1) 10 \lambda$ by $10 \lambda$ flat plate was computed by each of the four different models at $1 \mathrm{GHz}$. The physical optics solution with the near-field scattering equations (equations (4.4-1) through (4.4-7)), the non-coherent 'Mini-RCS' models (equations (4.5-2) and (4.5-3)), and both the two-dimensional (equations (4.3-15) through (4.3-18) and three-dimensional (equations (4.3-19) and (4.3-20)) uniform theory of diffraction solutions were computed in the forward scattering plane $\left(\phi_{\mathrm{S}}=90\right.$ degrees, $\theta_{\mathrm{s}}=0-90$ degrees) for an incidence angle of thirty degrees which was selected as a typical value to determine the scattering patterns. The solutions were computed for different combinations of transmitter and receiver distance from the plate.

The computed scattering patterns are compared to previously published results and each other in order to determine under what conditions each model is valid. The exact solution for the fields scattered by a finite width strip can be expressed as an infinite sum 
of Mathieu functions, or as integral equations [Ruc70]. Since these representations are unsuitable for numerical computation [Ruc70], the main lobe of the physical optics scattering pattern is used as a basis for determining the correct amplitude. A numerical solution using the method of moments could be used to determine the exact solution for the surface currents; however, this method is generally limited to plate sizes only one or two wavelengths long due to computational complexity [Rao82], [Bal89]. The physical optics solution is known to give the correct scattering in the specular direction [Ros66], [Ruc70], [Kno85]. Away from the specular region, the results in [Ros66] will be used to determine the validity of the different models. In [Ros66], the physical optics and geometrical theory of diffraction solutions for the far-field radar cross section of a flat plate were compared with measured data. The measured data indicate that the geometrical theory of diffraction is the most accurate scattering model for determining the radar cross-section of rectangular flat plates [Ros66]. Scattering patterns as a function of angle are computed for different combinations of source and observation distance. The distance is quantified in terms of a far-field distance parameter $\Delta$. The parameter $\Delta=\frac{r}{2 L^{2} / \lambda}$ is a normalized measure of the distance from the scatterer, where $L$ is the largest linear dimension of the scatterer. The division between near-field and far-field occurs at a distance of $r=r_{f f}=\frac{2 L^{2}}{\lambda}$. Hence $\Delta=1$ denotes the transition point between near-field and far-field observation locations. Observations are computed at distances of $\Delta=0.1$ through $\Delta=10.0$. The solutions considered are for TE polarization with respect to the perfectly conducting scatterer. The geometry indicated in the figures is for flat plates oriented parallel to the ground. When the polarization is TE, this corresponds to horizontal polarization. When the polarization is TE and the flat plate is oriented vertically like building faces or walls, this result corresponds to vertical polarization.

Inside buildings, receivers are nearly always in the near-field of scattering objects. For example, in Whittemore Hall on the Virginia Tech campus, there is a wall 29 meters long. For a carrier frequency of $4.0 \mathrm{GHz}$, a receiver would have to be over $22 \mathrm{~km}$ away to be in the far-field. A smaller wall encloses the end of the hallway. The length of this wall is 3 meters. A receiver would have to be 248 meters away to be in the far-field at $4.0 \mathrm{GHz}$. The length of the hallway is only 53 meters, and all receivers located in the hallway are in 
the near-field of the scattering wall. These two examples indicate that inside buildings, farfield assumptions for the behavior of the scattered field do not apply, and the solutions must be computed in the near-field.

The geometry for the computed scattering patterns is given in Figure 4.6-1. The distance from the transmitter to the center of the plate is denoted by $t$, and the distance from the center of the plate to the receiver is denoted by $r$. The distance $r$ is presented in terms of the far-field distance parameter $\Delta$. The scattered power is plotted in terms of decibels relative to the received power over a one meter free space path. Figure 4.6-2 shows the far-field scattering for a transmitter 300 meters from the plate, and $\Delta=10.0$. For the far field case in Figure 4.6-2, the main lobe of the PO solution and the main lobe of the twodimensional UTD solution converted to three-dimensional fields have the same amplitude. The three-dimensional UTD solution is incorrect since the length of the strip in the transverse direction cannot be ignored. Away from the specular direction, the UTD solution is more accurate than the PO solution since edge effects not accounted for in PO become significant. The deep nulls in the physical optics solution are due to complete phase cancellation at those scattering angles. However, the physical scattering mechanism is not due to phase, but edge effects. Therefore, away from the specular direction, UTD should be considered more accurate. The non-coherent model presented in [Sch92] is denoted by NonCoherent 1 and the area-dependent model is denoted by Non-Coherent 2 . The non-coherent models predict more scattered energy in the specular direction than the other models. This occurs because the non-coherent models assume a perfect reflection from an infinite planar surface if the specular point lies within the boundaries of the actual surface. As the actual size of the scatterer is reduced from infinity, the angular area that the scatterer occupies decreases. When the scatterer is of finite size and far away from the transmitter, the angular area that it occupies is significantly smaller than an infinite planar surface, and much of the energy that would have been scattered in the specular direction is scattered in other directions.

In Figure 4.6-3, both the transmitter and receiver are moved in by a factor of 10 $(t=30, \Delta=1.0)$. As the transmitter and receiver move closer to the scatterer, the angular area 

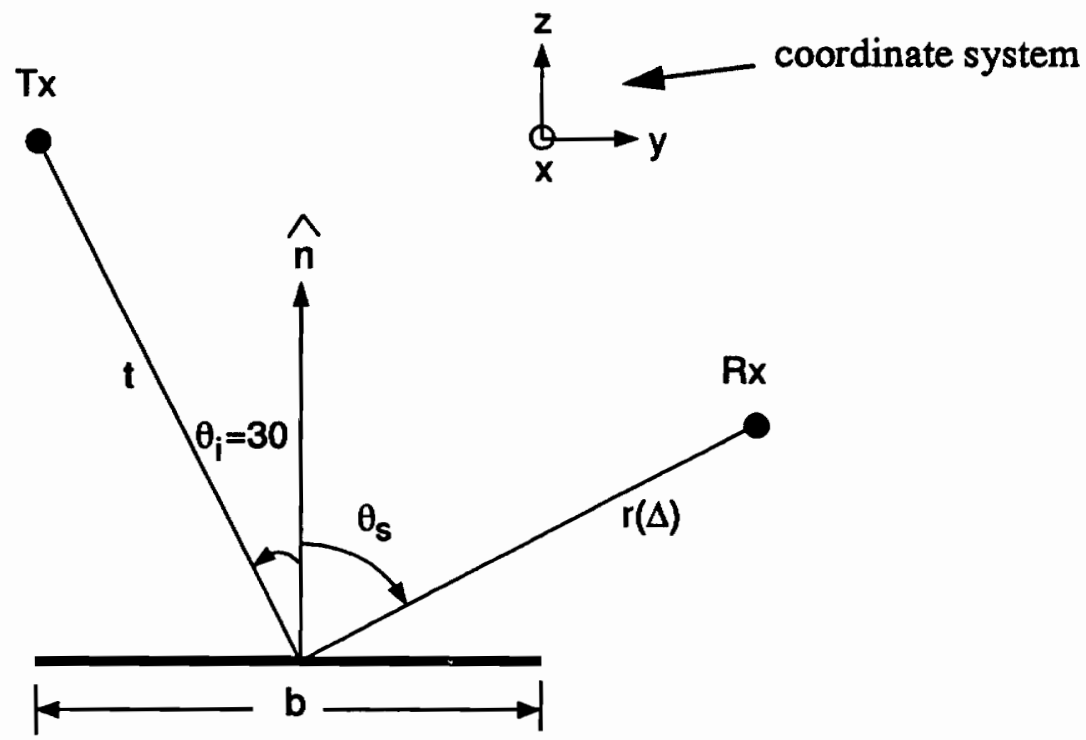

FIGURE 4.6-1. Assumed geometry for computing scattering patterns in the principle plane as a function of scattering angle from the surface normal.

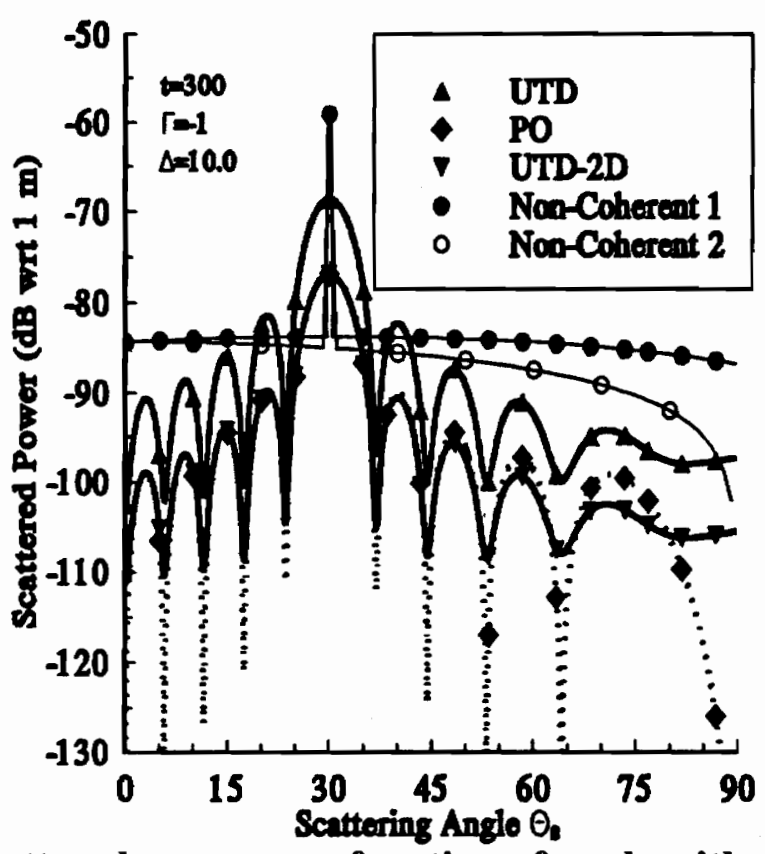

FIGURE 4.6-2. Scattered power as a function of angle with respect to the surface normal for four different scattering models. Both the transmitter and receiver are in the far-field of the scatterer.

occupied by the scatterer increases and both the reflection coefficient approximation and the three-dimensional UTD solution improve. However, the far-field two-dimensional 
solution still gives more accurate results since the far-field conversion formula of equation (4.3-18) is still valid and the power in the direction of the main lobe is closest to the physical optics solution. The non-coherent scattering model more accurately accounts for power in the main lobe about the specular direction than in the case shown in Figure 4.6-2.

Figure 4.6-4 shows the scattered power as a function of scattering angle for the four different models when both the transmitter and receiver are close to the scatterer $(t=3$, $\Delta=0.1)$. The far field conversion of the UTD fields from two dimensions to three dimensions is no longer valid. In fact, this model predicts a scattered power about $10 \mathrm{~dB}$ greater than the true solution. The three-dimensional UTD model approaches the PO solution since the transverse direction 'appears' nearly infinite when the receiver is so close to the scatterer. In addition, the non-coherent models are within a few $\mathrm{dB}$ of the near field calculation for scattering angles up to 65 degrees away from normal incidence. The scattering surface appears to be nearly infinite for this case and it appears that a geometrical optics model is a good approximation for the scattering when both the transmitter and receiver are close to the planar surface. This is nearly always the case for walls in buildings, and indicates that a geometrical optics model is appropriate for predicting in-building propagation. The addition of a three-dimensional UTD term eliminates the field discontinuity and correctly accounts for scattered energy in non-specular directions. In the far-field, the length of the plate in the transverse direction 'appears' small, and a far-field conversion factor may be used to account for the finite length of the plate. When the observation is close to the scatterer, the length of the plate approaches an infinite strip and 'appears' much larger. Hence, the length of the strip may be ignored when the receiver is close to the diffracting edge. This corresponds to the wall height for diffraction from a corner for inbuilding propagation prediction.

Figure 4.6-5 compares the scattering in the backscatter direction $\left(\theta_{\mathrm{i}}=\theta_{\mathrm{S}}, \phi_{\mathrm{i}}=\phi_{\mathrm{S}}\right)$ as computed with physical optics, the far-field two-dimensional uniform theory of diffraction, and the non-coherent 'Mini-RCS' model presented in [Sch92]. The first four sidelobes of the PO and UTD solutions are identical. Beyond the first four sidelobes, edge effects can be seen, and the correct backscattered power is given by the UTD solution. The 


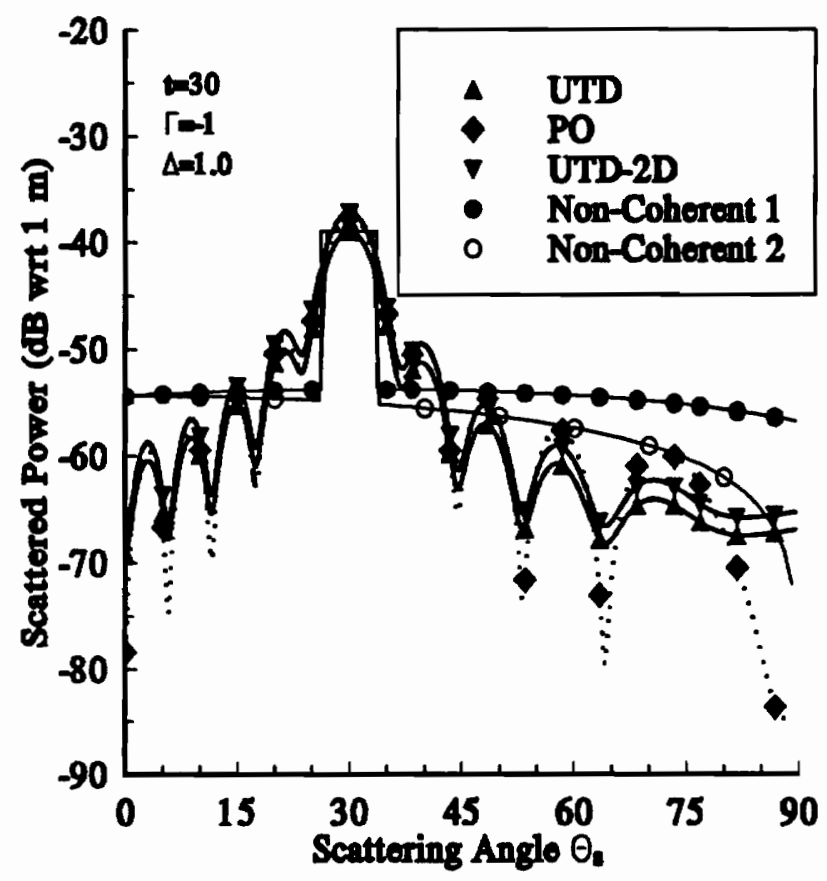

FIGURE 4.6-3. Scattered power as a function of angle with respect to the surface normal for four different scattering models. Both the transmitter and receiver are an order of magnitude closer to the scatterer than in Figure 4.6-2.

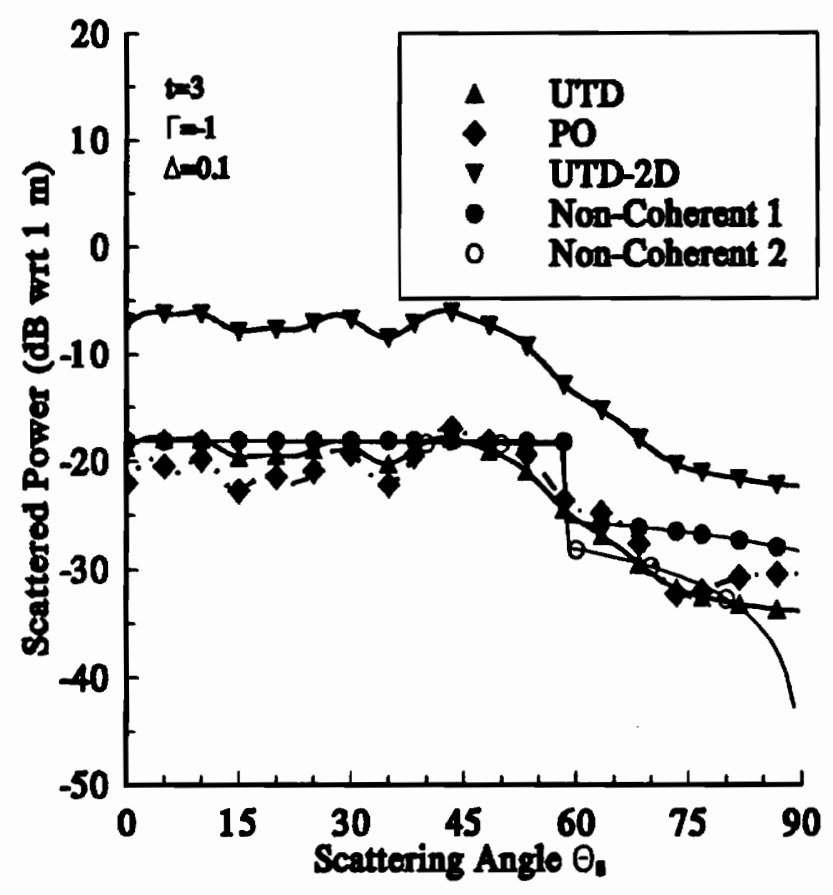

FIGURE 4.6-4. Scattered power as a function of angle with respect to the surface normal for four different scattering models. Both the transmitter and receiver are in the near field of the scatterer. 
non-coherent model used in [Sch92] is highly inaccurate for backscatter computations. The results in Figure 4.6-5 compare favorably with results computed and measured in [Ros66] for backscattering from a flat plate with the uniform geometrical theory of diffraction. Although backscatter is not a scattering case that is of primary importance in propagation for wireless communication system design, the inaccuracies of the non-coherent model and the physical optics model for large scattering angles indicates that these models can only be used in the appropriate region of validity.

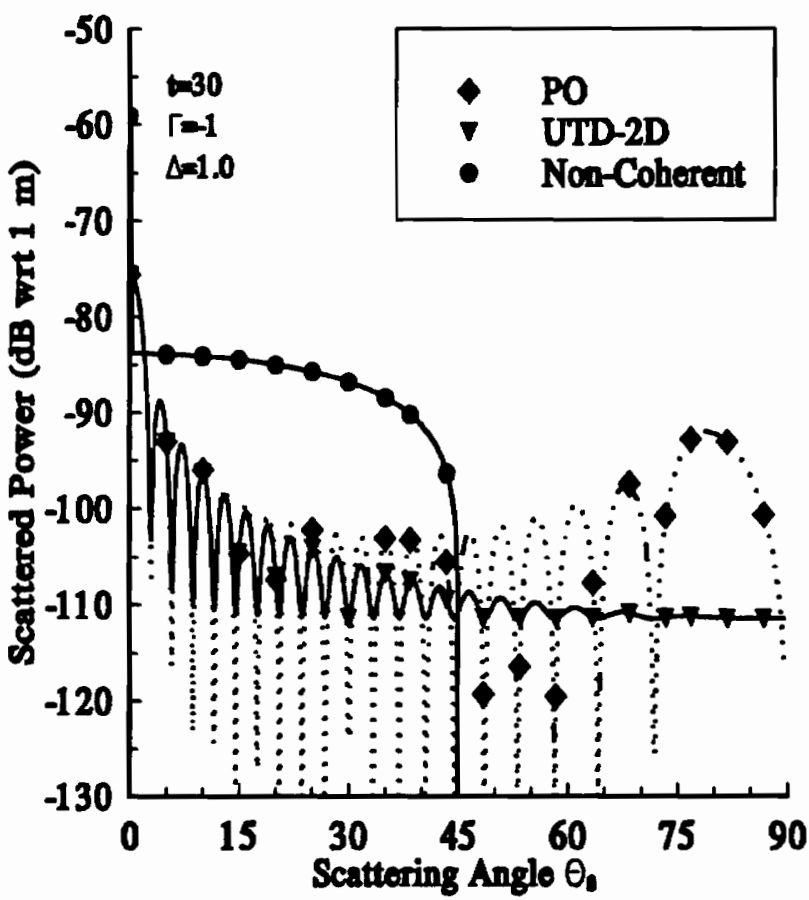

FIGURE 4.6-5. Backscattering as a function of incidence and scattering angle $\left(\theta_{i}=\theta_{s}\right)$ for three different scattering models.

\subsubsection{Implementation and Complexity Issues}

Practical application of each of these models in a site-specific propagation tool requires an understanding of the complexity and execution times of the different models. Both the physical optics and the non-coherent bistatic radar cross-section models are integrative techniques that require a computation for each integration patch (surface facet). Although these computations lend themselves to a ray tracing approach for determining the different integration patches, the physical optics requirement explained below that the 
surface patches be smaller than $\lambda / 2$ by $\lambda / 2$ places a restriction on the minimum tessellation frequency that can be used. Hence, although each computation requires little computational effort, the total computation time required can be significant. The uniform geometrical theory of diffraction model appears to be more complex than the other scattering models. However, the uniform geometrical theory of diffraction is an extension to geometrical optics that includes diffracted rays. For each diffracting edge (or corner), the specific ray path must be found only once and only one set of computations are required. Although these computations require the solution of a Fresnel integral, a polynomial approximation for small arguments and a polynomial approximation for large arguments can be used to numerically determine the integral. These approximations along with interpolation between the large and small argument polynomial estimates are implemented according to the algorithm in [Bal89]. Therefore, although the diffraction method is more complex to implement, it is more computationally efficient than the integrative methods of physical optics and non-coherent bistatic radar cross-section models.

\subsubsection{Scattering Patterns as a Function of Different Parameters}

\section{How to Interpret an Image Plot}

Image plots are used for qualitative comparison of scattering patterns as a function of two different variables. The intensity is proportional to the magnitude of the scattering in $\mathrm{dB}$ and is normalized to the maximum value in the plot. All scattering patterns as a function of angle were determined with the plane of scattering identical to the plane of incidence unless specified otherwise. Light spots are maxima, and dark spots are minima. The scattering dependence on one of the two variables can be examined by holding the other variable constant. That is, the scattering pattern may be examined by mentally drawing either a horizontal or vertical line along the plot and following the scattering pattern along that line. For each image plot, the range of values for each parameter is displayed along each axis. 


\section{Effect of integration patch size}

In order to determine the minimum resolution required for integration using the physical optics method, the integration patch size was varied for a $10 \lambda$ by $10 \lambda$ square plate. For square patches with lengths less than $\lambda / 2$, there is no noticeable difference in scattering pattern as a function of angle for all incidence angles. Hence, when scattering is implemented by physical optics in site-specific propagation prediction programs, the size of the wavefront on the surface of the scatterer should be no larger than $\lambda / 2$ by $\lambda / 2$ for accurate computation of scattering patterns for both near and far field illuminance and observation distances.

\section{Near Field vs. Far Field}

Since it is possible for receivers to be in the near field of scattering objects, the scattering pattern is examined as a function of observation distance for different angles of incidence. In Figure 4.6-6, the upper left plot shows the physical optics scattering pattern from a perfectly conducting plate for an incidence angle of zero degrees. The incidence angle in the upper right plot is 30 degrees, and the incidence angles for the bottom two are 60 and 80 degrees. Figure 4.6-1 shows the geometry for an incidence angle of 30 degrees. For all cases, the flat plates were illuminated with a spherical wave at a distance of 300 meters. Notice that in the far field $(\Delta>1)$, the maximum of the scattering pattern at a constant fixed distance is in the specular direction $\left(\theta_{\mathrm{s}}=\theta_{\mathfrak{i}}\right)$. This can be seen by mentally drawing a horizontal line for a constant $\Delta>1$ in each plot in Figure 4.6-6. The maximum value (brightest spot) along this line occurs in the specular direction. These patterns are the typical $\sin (\mathrm{x}) / \mathrm{x}$ shaped patterns for far-field scattering from a flat plate. The direction of the main lobe changes as a function of incidence angle, but the general $\sin (\mathrm{x}) / \mathrm{x}$ scattering pattern is retained. In the near field, however, the scattering patterns are not 'intuitive' as the contributions from different parts of the surface combine to create scattering patterns that have significant energy in directions other than specular. This is likely because the specular point for the combination of transmitter and receiver location lies on the plate as shown in Figure 4.3-8. The scattering patterns computed using UTD for the same four cases as Figure 4.6-6 are shown in Figure 4.6-7. As could also be seen in Figure 4.6-2, the nulls are 
deeper for the physical optics solution than for the UTD solution. When flat dielectric plates are considered, the amplitude of the scattering is affected, but the general $\sin (x) / x$ shape of the scattering pattern is maintained. 

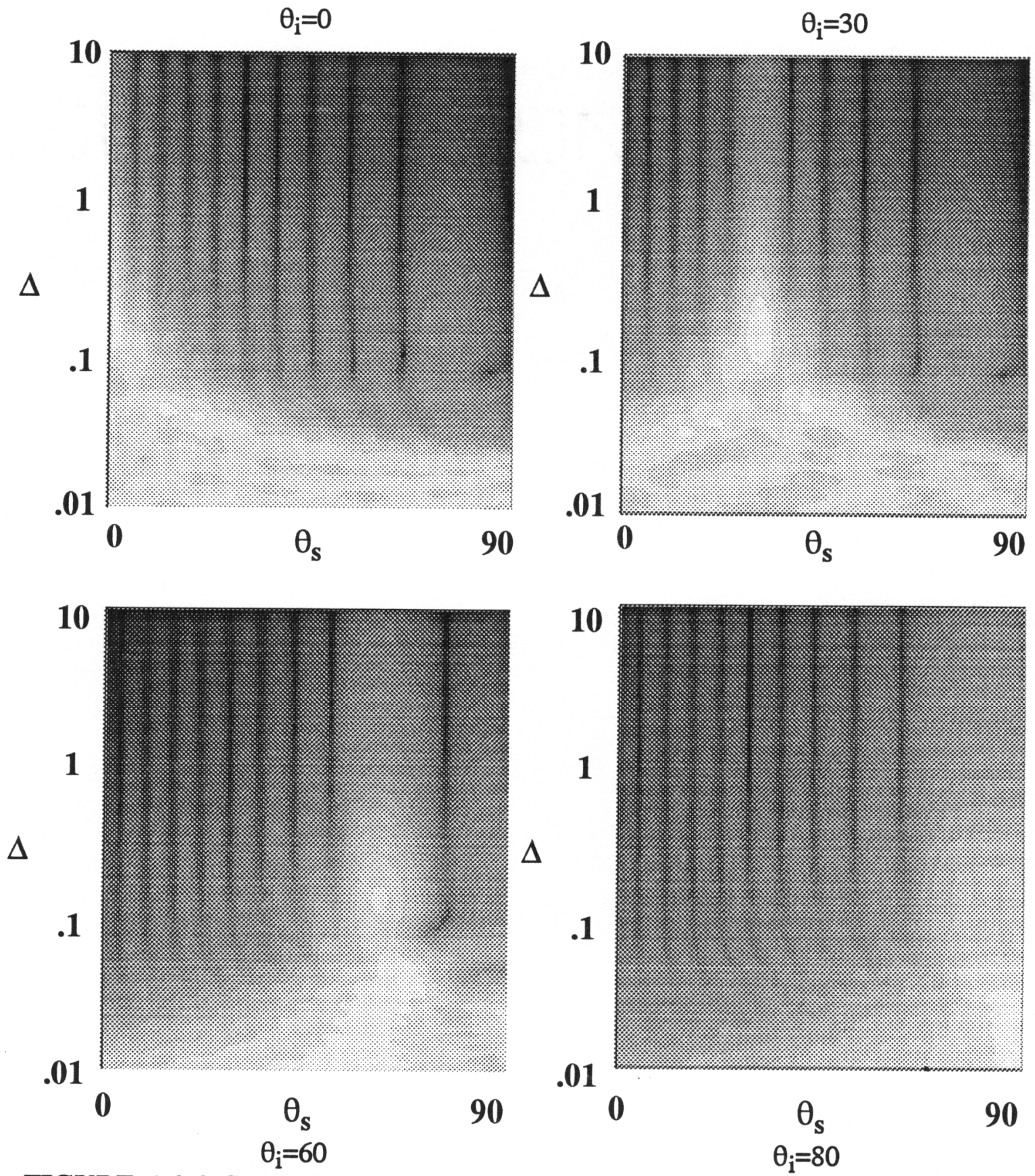

FIGURE 4.6-6. Scattering patterns of a PEC plate as a function of observation distance and scattering angle for incidence angles of $0,30,60$, and 80 degrees computed with physical optics. Increasing intensity denotes increasing scattered power. Note that for $\Delta>1$, the maximum scattered power is in the specular direction $\left(\theta_{\mathrm{s}}=\theta_{\mathrm{i}}\right)$. 
Figure 4.6-8 shows the four scattering patterns computed using the non-coherent scattering model. Notice that the scattered power is very strong near the specular direction and then falls off sharply when no specular field is received. In addition the scattering pattern that was predicted with the uniform geometrical theory of diffraction and has been observed in measurements [Ros66] has not been predicted. Hence, the applicability of this model in the far-field is questionable at best. In the near field, Figure 4.6-4 showed that the scattered power away from the specular direction is within about $5 \mathrm{~dB}$ of that predicted by the other methods, but the shape of the scattering pattern is not predicted. This result is not surprising since neither phase effects or the contributions of edges are included in the heuristically developed non-coherent models. 


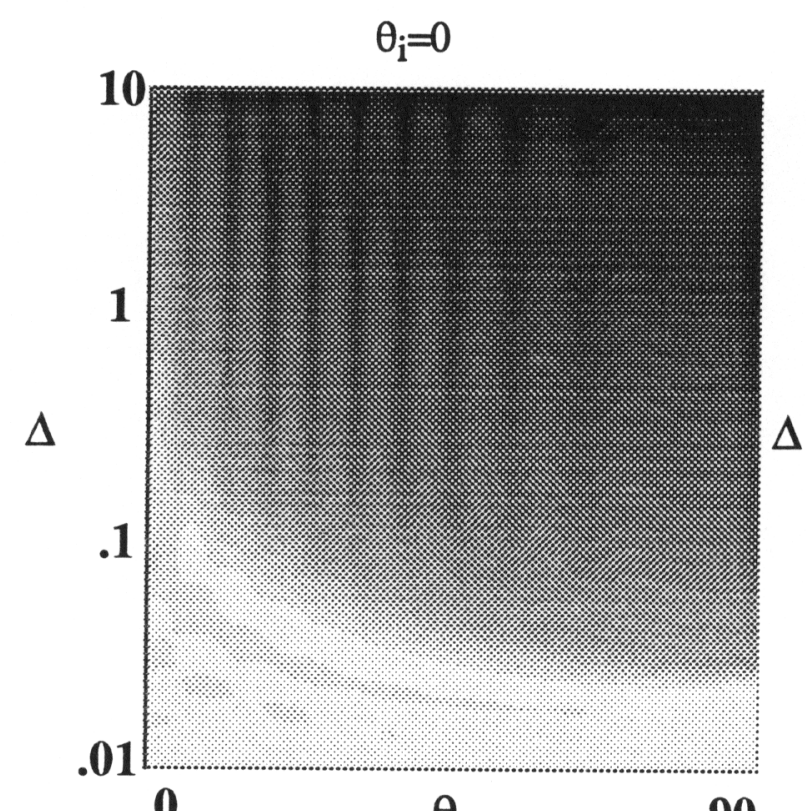

$\Delta$

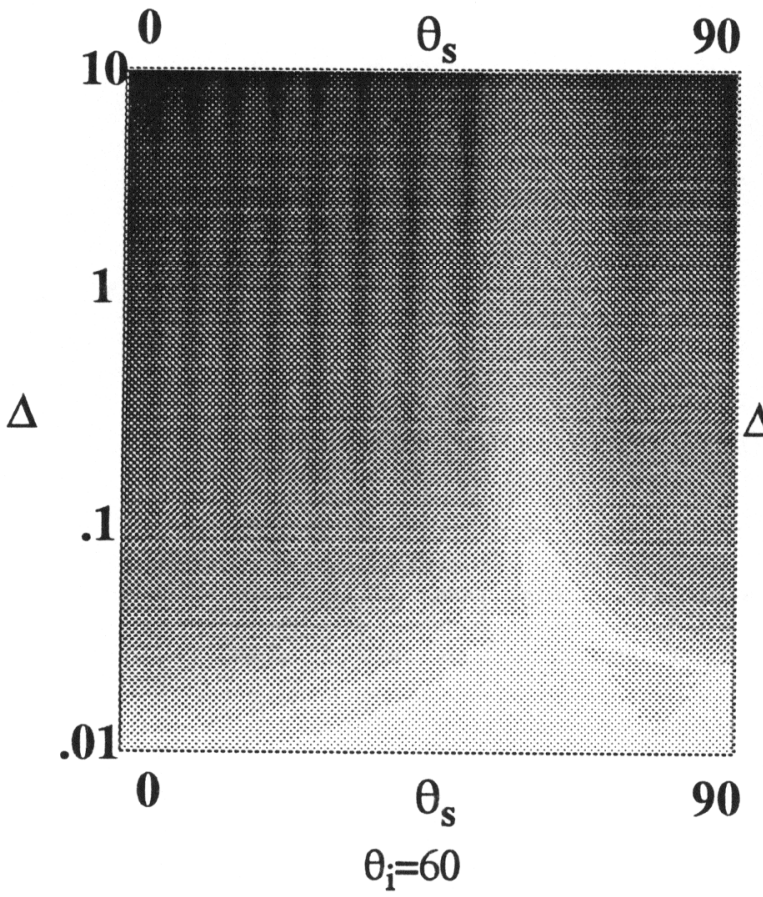

$\theta_{\mathrm{i}}=30$

$\Delta$

1

.1

01

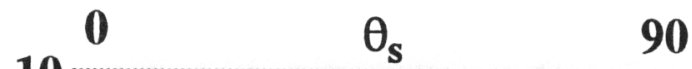

1

$\Delta$

.01

0

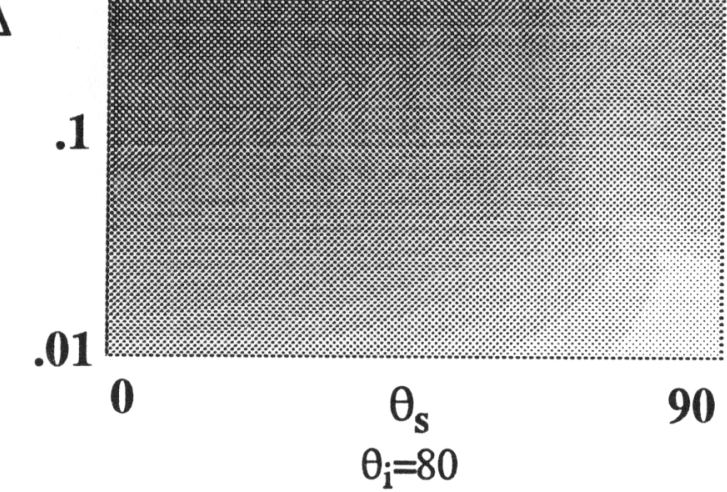

FIGURE 4.6-7. Scattering patterns of a PEC plate as a function of observation distance and scattering angle for incidence angles of $0,30,60$, and 80 degrees computed by the uniform theory of diffraction. Increasing intensity denotes increasing scattered power. Note that for $\Delta>1$, the maximum scattered power occurs in the specular direction $\left(\theta_{\mathrm{i}}=\theta_{\mathrm{S}}\right)$. 

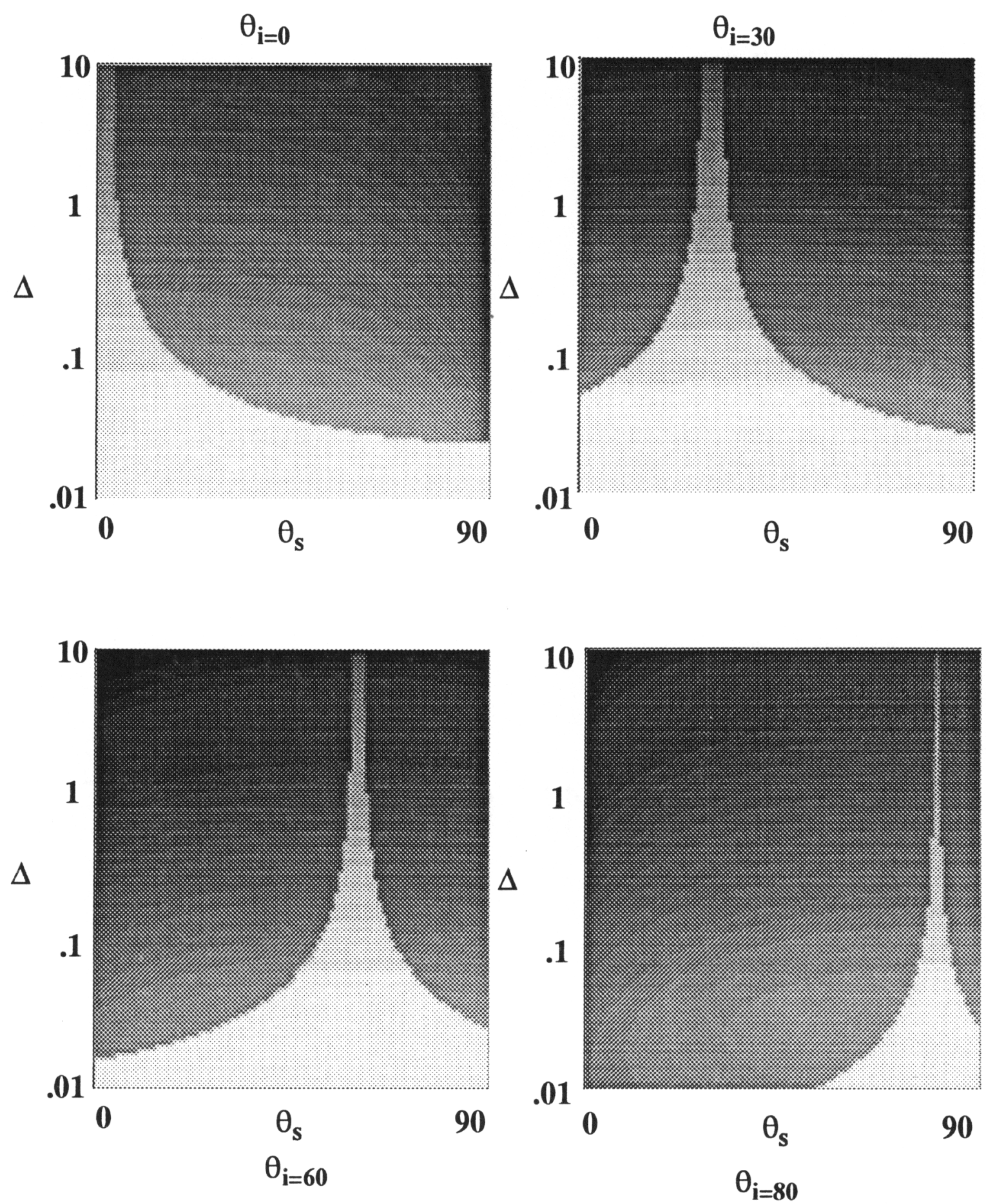

FIGURE 4.6-8. Scattering patterns of a PEC plate as a function of observation distance and scattering angle for incidence angles of $0,30,60$, and 80 degrees. computed by the non-coherent scattering model. Increasing intensity denotes increasing scattered power. Note that the maximum scattered power always occurs in the specular direction $\left(\theta_{\mathrm{i}}=\theta_{\mathbf{S}}\right)$. 


\section{Effects of Surface Roughness on Reflection Coefficient}

For scattering from dielectric surfaces, the scattered power depends on the Fresnel plane wave reflection coefficients. In the geometrical optics, geometrical theory of diffraction, and non-coherent scattering models, this effect appears directly in the scattered power since both the reflected field and the diffraction coefficients depend on the reflection coefficient. In the physical optics model, the postulated surface currents depend on the reflection coefficient, and this in turn affects the scattered field. When a surface is not perfectly smooth, the coherent scattering in the specular direction is reduced. If the surface roughness is not too large, the coherent nature of the specularly reflected field is not lost. The average Fresnel reflection coefficient becomes [Ame53], [Bec63], [Ruc70]

$$
\bar{\Gamma}=\Gamma e^{-2\left(k_{0} h \cos \theta_{i}\right)^{2}}
$$

where $k_{0}$ is the wavenumber $(2 \pi / \lambda), h^{2}$ is the mean square roughness height $\left(h^{2}=<\xi^{2}(x, y)>\right.$ where $\xi$ is the distribution of surface heights as a function of position on the surface). An image plot of the rough surface correction factor for the reflection coefficient is given in Figure 4.6-9. The correction factor has a maximum of 1.0 for perfectly smooth surfaces, and perfect grazing angles. For slight roughness and incidence away from grazing, the amount of coherent scattering in the specular direction is decreased. Corrected reflection coefficients could be used to account for slight surface roughness in an in-building propagation prediction model. In the models presented here, the surfaces are assumed to be perfectly smooth. 


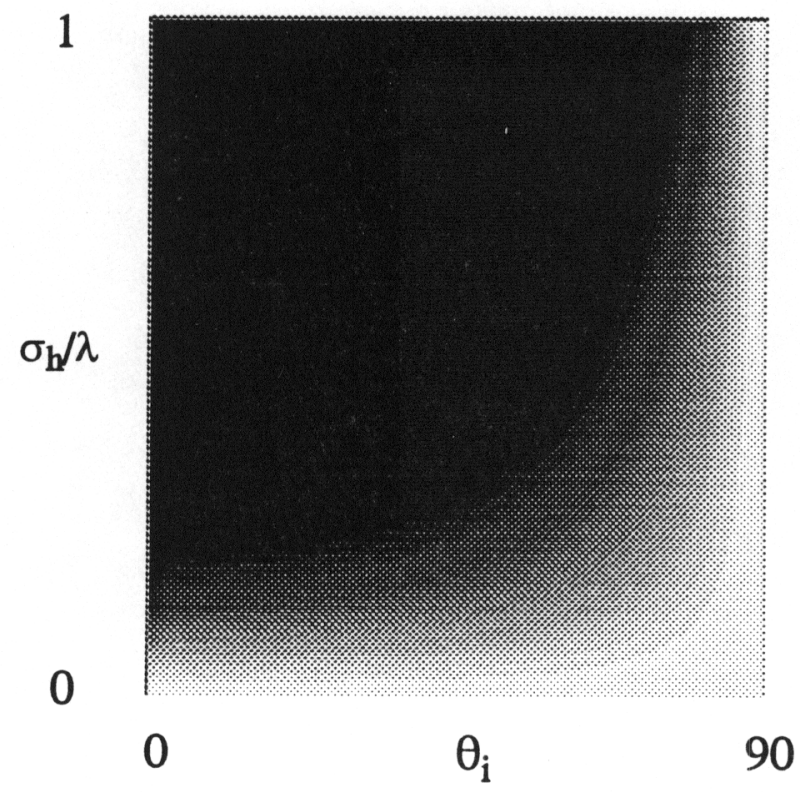

FIGURE 4.6-9. Reflection coefficient correction factor for slightly rough surfaces as a function of surface roughness in wavelengths and incidence angle. For slightly rough surfaces $\left(\sigma_{h} / \lambda<0.2\right)$, this correction factor can be used to calculate the reduction of coherent power in the specular direction.

Reflection coefficients for several rough surfaces were measured on the Virginia Tech campus in [Lan92]. Limestone, glass, and brick walls were measured outdoors with a spread spectrum measurement system that could resolve the time delays between the direct and reflected multipath components. Measured reflection coefficients at both 1.9 and $4.0 \mathrm{GHz}$ were found to be bounded by the theoretical smooth surface reflection coefficient and the modified rough surface reflection coefficient [Lan92], [Sou92].

\subsection{Summary}

A geometrical optics (GO) model assumes that electromagnetic energy travels in straight lines and changes directions only when reflected or refracted by a surface. The amplitudes of the reflected and transmitted fields are determined by the Fresnel plane wave reflection and transmission coefficients. The accuracy depends on the relative size of the scatterer with respect to wavelength. Physical optics (PO) assumes that a particular 
surface current is induced on the surface of the scatterer in the geometrical optics lit region. This equivalent surface current is then integrated to determine the scattered fields. The accuracy of the scattered fields depends on the validity of the assumed surface current on the scattering body. The physical optics solution is most accurate in the specular direction. Scattering in directions other than specular is influenced by the edges since, it is near the edges that the assumption for the current is least accurate. Both GO and PO include the phase of the fields. A non-coherent 'Mini-RCS' method supplements the GO fields by adding scattered energy from surface patches that each act as independent bistatic radar scatterers. As this is a heuristic model, the accuracy is as yet undetermined, but the results of numerical calculations indicate that it may be a useful model for near-field scattering. This model may also be useful when small amounts of surface roughness are added to the model [Ogi91]. The uniform geometrical theory of diffraction (UTD) supplements the GO solution by adding diffracted rays that account for energy in non-specular directions and eliminate the field discontinuities at the incident and reflection shadow boundaries.

The solution for scattering from a finite sized smooth flat plate as computed from the physical optics surface current assumption is most accurate in the specular direction. Away from the specular direction, the solution breaks down because contributions from the unmodeled edges become the dominant scattering mechanism. In order for the physical optics solution to be used, integration patch sizes should be less than $\lambda / 2$ in both length and width. The two-dimensional uniform geometrical theory of diffraction (UTD) solution based on a finite strip model has been developed for far-field observation distances $(\Delta \geq 1.0)$ for all incident and scattering angles when a conversion factor that considers the finite length of the scatterer is used to convert from two-dimensional to three-dimensional fields. When the observation distance is in the near-field $(\Delta \leq 1.0)$, the three-dimensional UTD solution of the infinite strip with the far-field geometrical optics fields model is used. It is desirable to use the UTD solutions since these solutions include the polarization of the incident and scattered field in the model. The physical optics and non-coherent models do not include polarization effects. The simple incoherent power summation model proposed in [Sch92] and the other non-coherent model do not provide accurate results for far-field observation distances. However, in the near-field these models predict the scattered power 
within about $5 \mathrm{~dB}$. For all distances, however, the non-coherent models do not predict the scattering pattern as a function of angle that is predicted by the uniform geometrical theory of diffraction. The UTD solution was shown to agree with measured radar cross-sections in [Ros66]. Since this non-coherent models are much simpler than the others, it is likely that they may find application in numerical computation algorithms where computation speed is critical and the scattering must be determined when the receiver is in the near-field of the scatterer.

This chapter has presented several methods for determining the scattering from rectangular flat objects. These objects can be used to model walls inside a building or the exterior walls of a building in a microcellular environment. The uniform geometrical theory of diffraction was shown to give accurate results with both the infinite strip and the finite strip model where the implementation is dependent upon the observation distance from the scattering object. Hence, it is this uniform geometrical theory of diffraction model that is used for site-specific in-building propagation prediction using ray tracing as is subsequently described in Chapter 5. 


\section{Site-Specific In-Building Propagation Prediction}

\subsection{Overview}

Buildings vary greatly in size, shape, and type of construction materials. This can make propagation prediction difficult. As shown in Chapter 3, measured propagation statistics vary greatly from building to building and only broad conclusions related to the building type can be made (recall Table 3.1-1). This chapter presents a ray tracing method to predict the power delay profile estimate of the channel impulse response. From the power delay profile, parameters such as path loss and time delay spread of indoor radio channels may be determined.

The field of graphical ray tracing for creating a two-dimensional picture of a threedimensional world is well developed [Gla89], [Ste90]. An object or group of objects called a scene are described in terms of their geometry and light scattering properties (color). The computer attempts to recreate a photograph of the scene for a fixed observer and one or more light sources. The graphical ray tracing is a geometrical optics model for light. To take advantage of the similarities of the graphical ray tracing and the geometrical optics models, the source code for the graphical ray tracer in [Ste90] has been extensively modified to generate propagation impulse response data instead of pixel color. This geometrical optics model for electromagnetic wave propagation in buildings is used to predict multipath power delay profiles.

Ray tracing represents the high-frequency limit of the more rigorous field problem and can give quick approximate solutions when more elaborate methods are unworkable. Here, a geometrical optics model is used to predict the propagation of radio waves in buildings. Ray tracing is a physically tractable method of predicting the delay spread and path loss of in-building radio signals. The time delays of individual multipath components can be linked to specific radio paths. This concept is similar to radar systems that use the time delay to estimate the distance to a target. Ray tracing methods have been proposed for propagation prediction in microcellular environments [Ike91], [Ros91], [Tak91], [Ros92], [Sch92] and for modeling propagation in rough terrain [Bis88]. Preliminary 
results from ray tracing in buildings using image theory at $18 \mathrm{GHz}$ were presented in [McK91]. Ray tracing for indoor propagation has also been proposed in [Dri91], [Dri92], [Hon92], [Kia92], [Lau92], and [Law92]. However, none of these previous applications of ray tracing for in-building propagation prediction have compared measured and predicted wide band power delay profiles on a location-by-location basis. Yet, it is a location-bylocation comparison that is required to determine the accuracy of a ray tracing propagation model.

It is assumed here that large scale building geometry dominates the average path loss. Measurements show the individual resolvable multipath components fade only slightly (less than $5 \mathrm{~dB}$ peak-to-peak total multipath power variation) as a receiver is moved in indoor and outdoor channels over several wavelengths without major topography changes, or as the terminals remain stationary over several seconds [Gan90], [Gan91], [Te191]. Thus, this assumption seems reasonable. The time dispersion of a wide band channel is due to the location and orientation of the scatterers, and can easily be determined via ray tracing. The propagation model uses geometrical optics to trace the propagation of direct, reflected, transmitted and diffracted fields. The rays, which represent a local plane wave of the total field, originate from point sources and propagate in three dimensional space. The lack of significant difference in propagation characteristics throughout the low microwave band (Section 3.3 The Effects of Carrier Frequency on Propagation Characteristics) indicates that a geometrical optics model where the actual frequency dependence is small can provide accurate prediction for a variety of frequencies.

This chapter presents the details of the ray tracing model. First, the ray tracing computer code used to predict the propagation is described. The source ray directions and the interaction of rays with objects in the building database are discussed. The path loss dependence of direct, transmitted, reflected, and diffracted rays is discussed. The chapter concludes with the description of the algorithm for the identification of specular rays and a description of the data processing used to convert the raw ray tracing output to power delay profiles. 


\subsection{Building Blueprint Representation}

AutoCAD ${ }^{\mathrm{TM}}$ is used as the building database manager. This program was selected because it is considered an industry standard CAD package, and it is believed that building blueprint data may be readily available in an AutoCAD format. AutoCAD supports a standard drawing exchange format allowing data to be exported in ASCII format. This exchange format is used to export the building database directly from AutoCAD to the propagation prediction tool.

The representation of buildings in a computer database, even different buildings represented within AutoCAD, can vary significantly from building to building. This section discusses a 'standard' representation of the major building features inside the AutoCAD database manager, and explains how the AutoCAD building representation is converted to a file compatible with the ray tracing prediction program.

\subsubsection{Conversion of AutoCAD Data Format to Ray Tracing Input}

In order to implement site-specific propagation models, it is necessary to incorporate the site-specific building information into the propagation prediction tool. AutoCAD is used to represent the significant building features such as wall locations and building materials. For many of the academic buildings on campus, the Virginia Tech Facilities Planning and Construction in 331 Burruss Hall has the building blueprints available in an AutoCAD format. This building representation displays wall locations and many other levels of detail in two dimensions on different drawing levels. The AutoCAD representation must be in a particular format so that this data may be converted to the correct format for input into the ray tracing program. The blueprints available from Facilities Planning are not in the correct format. To obtain the correct format, an operator uses AutoCAD to manipulate the database. The operator must create new drawing layers, draw lines for the wall locations, provide a thickness (wall height) for each wall, and place the locations of transmitters and receivers in the building. First, extraneous information was deleted from the file. A BUILDING layer was created and wall locations were drawn on the BUILDING layer with the pline command. It is straightforward to draw the wall locations on the 
BUILDING layer while the original drawing that was drawn on another layer is displayed on the screen. Figure 5.1-1 shows the representation of the second floor of Whittemore Hall as obtained from Facilities Planning. This representation includes 'thick' walls, stairs, doors, and features such as stairs that are external to the building. The individual features of a building are quite complicated. Since a geometrical optics model is used, only objects that are much larger than a wavelength at microwave frequencies are represented. Hence, it is not practical to include every single small-scale feature within a building. Exit signs, door knobs, door hinges, and furniture are not included in the model. Only large objects such as building walls or office partitions are included in the database. Each wall is considered to be infinitely thin. The major features as drawn on the BUILDING layer in AutoCAD are shown in Figure 5.1-2.

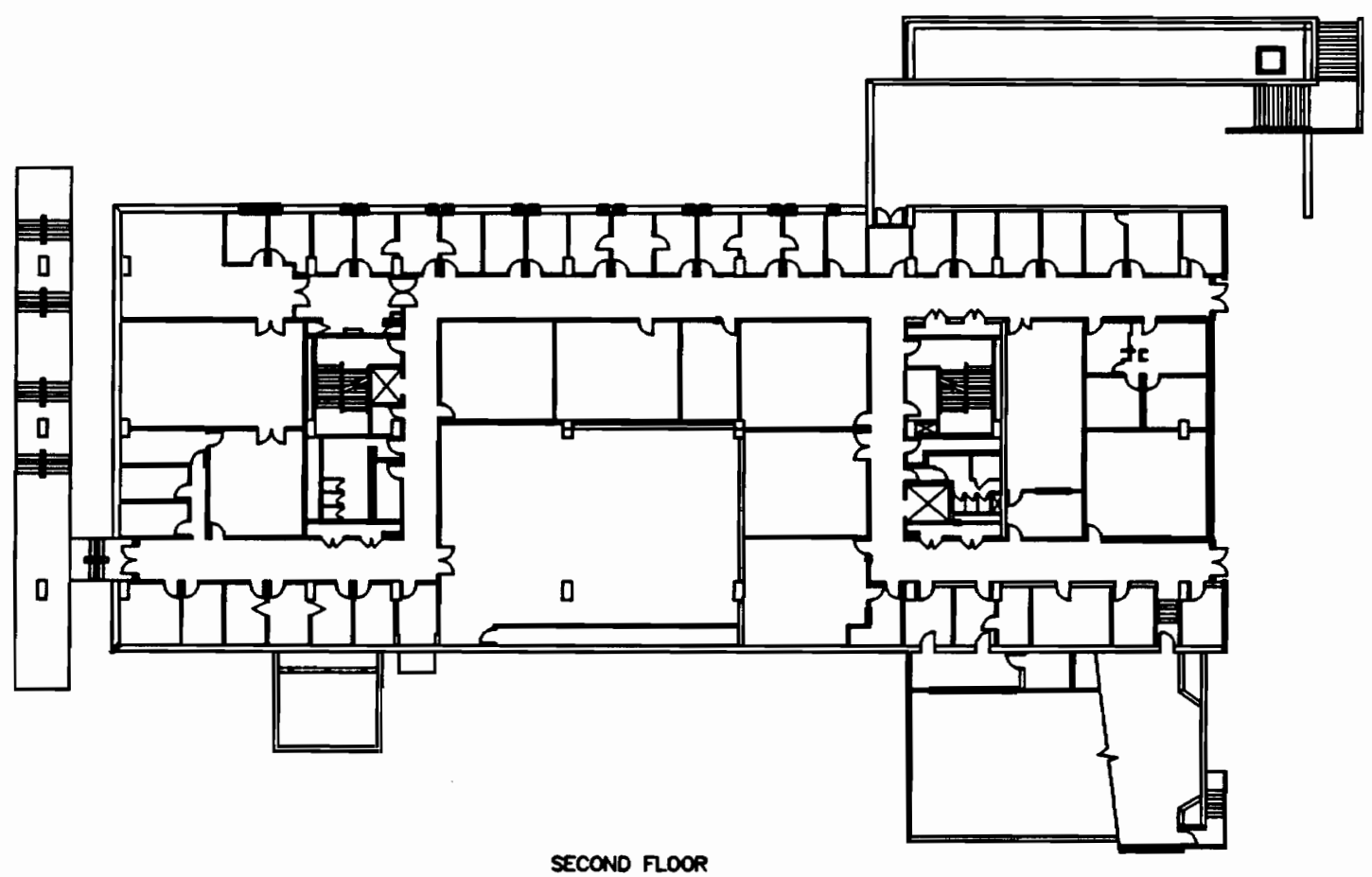

FIGURE 5.1-1. Example of the AutoCAD file as obtained from Facilities Planning and Construction.

The inclusion of only major large-scale objects is justified in that the goal of this work is to predict large scale average path loss and time dispersion as influenced by major changes in the geometry surrounding the transmitter and receiver. Once all major features 


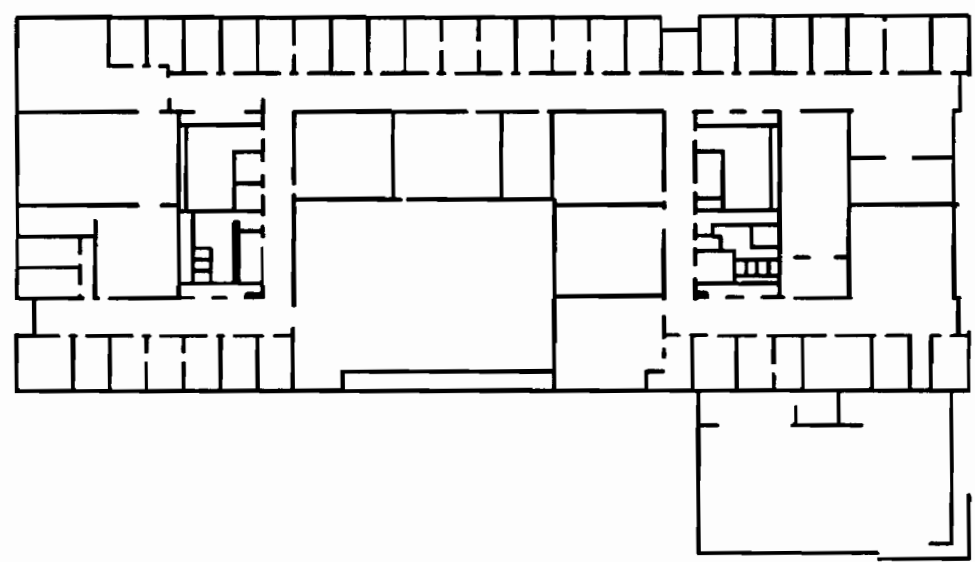

FIGURE 5.1-2. Two-dimensional representation of major building features drawn on the BUILDING later in AutoCAD.

such as wall locations are entered, the two-dimensional representation is extended to three dimensions in AutoCAD with the change command. The line thickness is changed from zero to a finite value to represent the wall height. All distances in AutoCAD are specified in English units. Figure 5.1-3 shows the three-dimensional extended view of the building blueprint in AutoCAD for the second floor of Whittemore Hall.

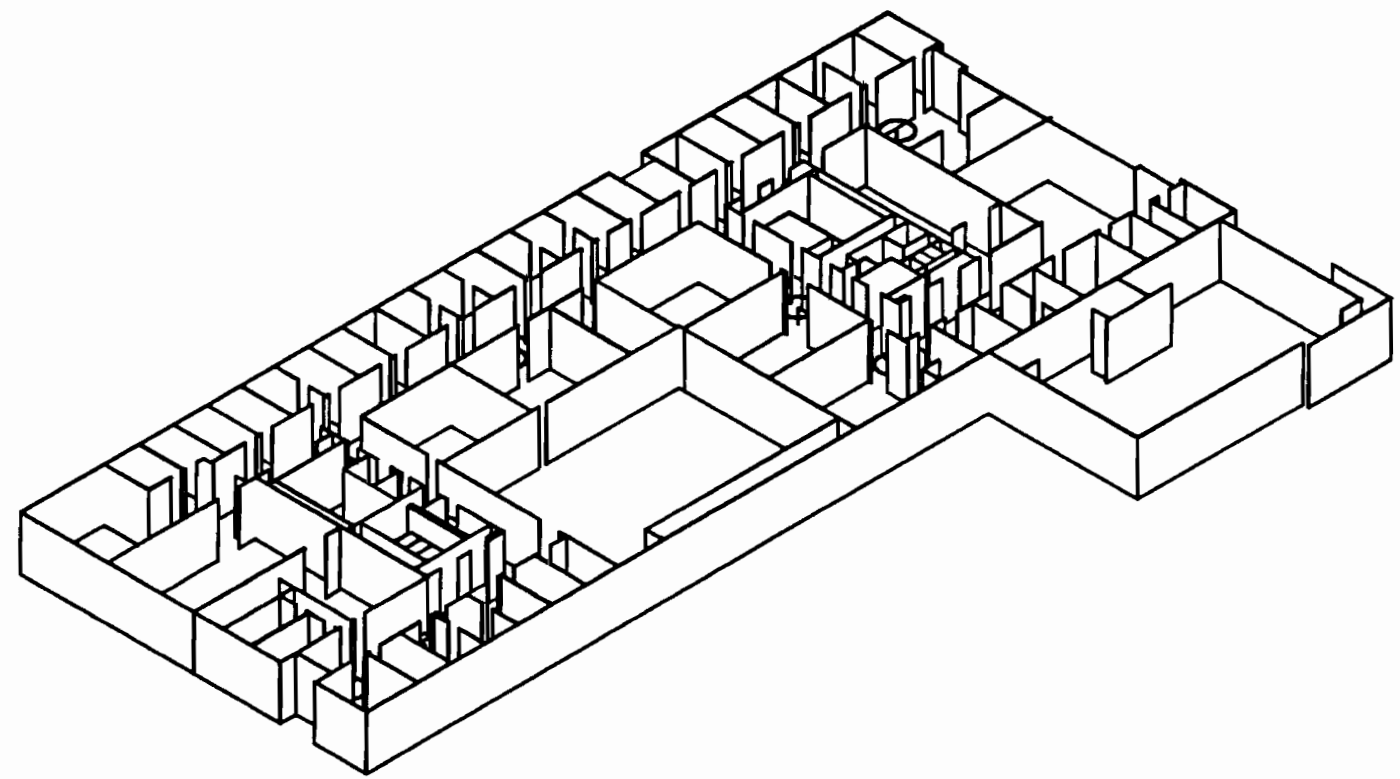

FIGURE 5.1-3. Three-dimensional AutoCAD representation of the second floor of Whittemore Hall on the Virginia Tech campus. 
A database conversion program developed and described in detail in [Sch92] is used to convert the AutoCAD information to a format that can be input directly into the ray tracing program. Once the AutoCAD file is in the correct format, the three-dimensional building blueprint information is exported from AutoCAD in a standard Drawing Exchange File (DXF) format. A conversion program written in C converts the DXF file to a 'ray' file that is ready for input to the ray tracing program. More information concerning the use of AutoCAD as a building database manager can be found in [Sch92].

\subsubsection{Transmitter and Receiver Locations}

For each run of the ray tracing prediction, exactly one transmitter location and at least one receiver location must be specified. Multiple receiver locations may be specified, and this is more efficient than running the program several times since the computation time per receiver decreases as more receiver locations are simulated even though the total simulation time is increased. Transmitter and receiver locations are specified as 'points' in three dimensions in AutoCAD on the TRANSMITTER and RECEIVER building layers, respectively.

\subsubsection{Ray Tracing Input File (.ray)}

The ray tracing program must be able to interface with a standard representation of the building blueprint information. This section describes a Ray Tracing Definition Language (RTDL) modified from the RTDL given in [Ste90]. An example of a subset of a ray tracing input file is given in Figure 5.1-4. This file is called a ray file and contains all the information necessary to run a ray tracing propagation prediction. The RTDL is not casesensitive since the input parser in the ray tracing program converts all characters to upper case.

Major planar building features such as walls are represented as parallelograms. Each PARALLELOGRAM is identified by three three-dimensional vectors. The first vector $l o c$ is the location vector from the origin to one corner of the parallelogram. The origin is simply a reference coordinate that is the default value given in AutoCAD. The vectors $v 1$ and $v 2$ identify the locations of the two corners of the parallelogram closest to the first 


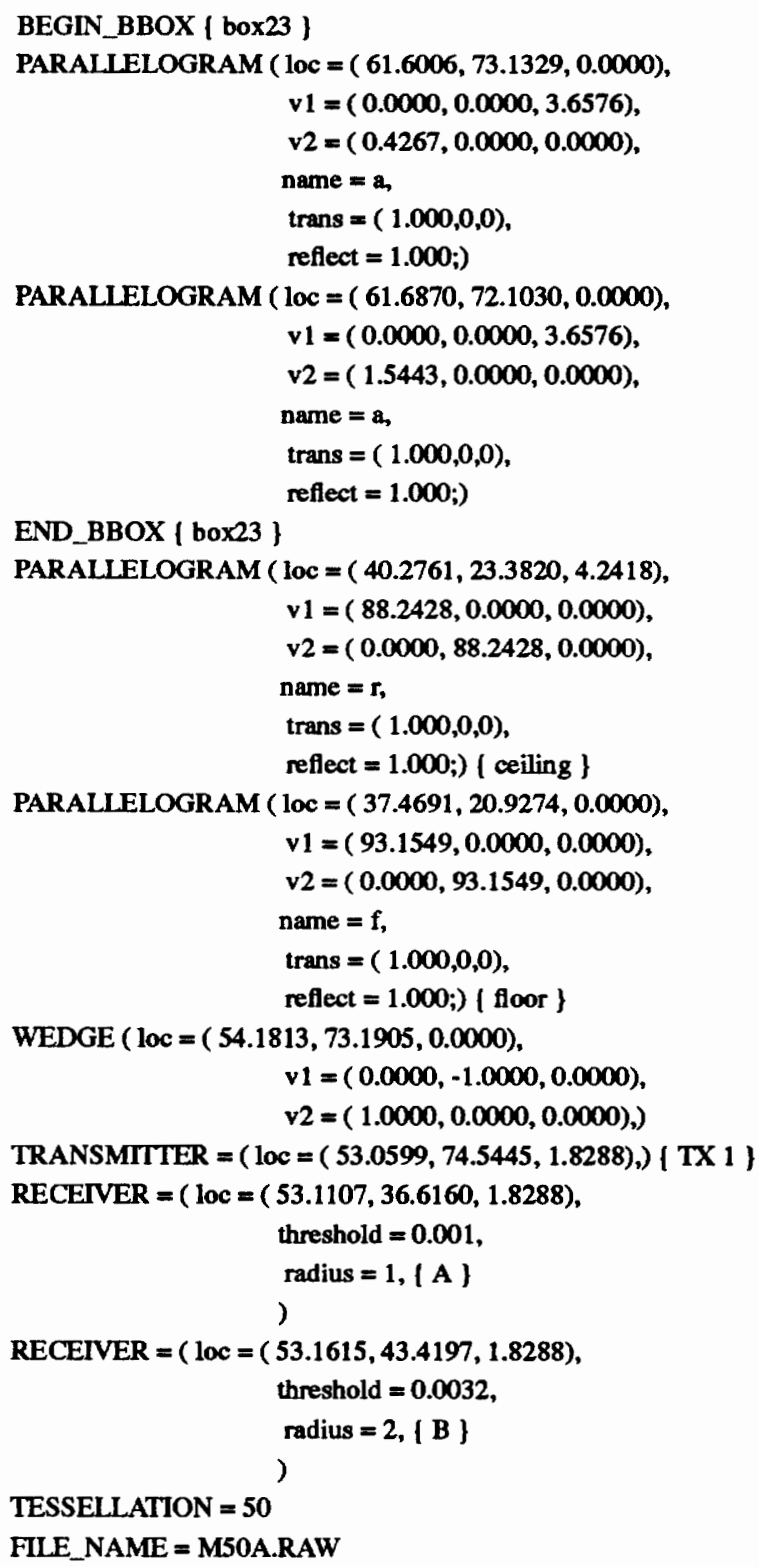

\section{FIGURE 5.1-4. Sample ray tracing input file (.ray).}

corner as indicated in Figure 5.1-5. The vector elements are the $x, y$, and $z$ components in meters. Also specified for each parallelogram is a name, and a transmission and reflection coefficient. For the implementation of the program as described here, the transmission and reflection coefficients are each set to 1.0 and are not used in the propagation prediction. 


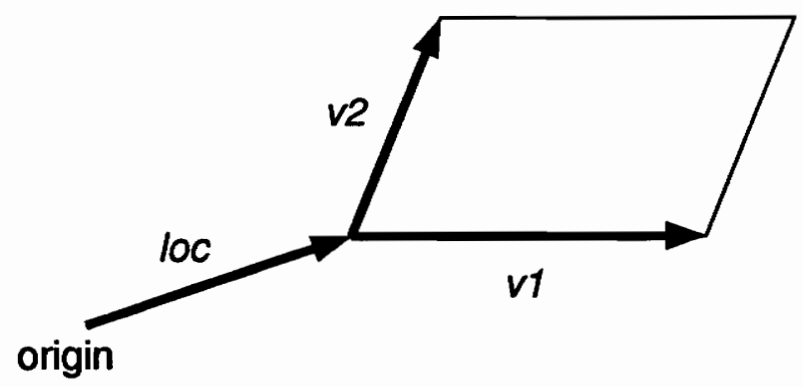

FIGURE 5.1-5. A building wall represented as a parallelogram. The location vector $l o c$ and parallel edge vectors $v 1$ and $v 2$ define the wall in the building database.

These parameters are included for potential future modification. The attenuation of rays upon reflection and transmission is modeled by the Fresnel plane wave reflection and transmission coefficients. These coefficients vary with incidence angle and are modeled according to the name of the object (parallelogram). Objects with the same name are considered to have identical electrical properties. The name must be a single alphanumeric character. Examples of values used include A, C, F, M, and R. Each letter indicates an object with different material properties than the others. The letter $A$ represents a drywall in Whittemore, the letter $\mathrm{C}$ represents a concrete wall, and the letter $\mathrm{M}$ represents a metal door at the end of a hallway in Norris Hall. The letters R and F indicate the ceiling and the floor, respectively. By giving each object a name that distinguishes the electrical properties of the material from the properties of the other materials, many different objects may be entered simply into the building database, and those properties may be altered quite readily.

The terms BEGIN_BBOX and END_BBOX in the ray file are used to place bounding volumes around groups of objects in the building database. Bounding volumes are used to decrease the number of intersection tests required to determine if a ray hits an object. Hierarchical levels of bounding volumes can be used to significantly reduce the amount of computational time required to predict the propagation. At present, the software user must determine which objects should be placed in bounding volumes. An efficient automatic method for determining which objects should be grouped with others inside bounding volumes would be an important development in the support of automated ray 
tracing for propagation prediction. Commented items in the ray tracing input file that are ignored by the ray tracing program are surrounded by curly braces, e.g. \{ box 23$\}$. The use of commented items facilitates editing the ray file if necessary.

At present, diffraction wedges must be manually specified as a block in the AutoCAD file. An automated procedure to determine diffraction wedges would be a useful modification to the propagation prediction tool. In the ray file, a diffraction wedge is specified as a WEDGE with a location vector and two direction vectors $v 1$ and $v 2$. The unit vectors $v 1$ and $v 2$ specify the directions of the wedge boundaries as they extend away from the corner.

The transmitter is completely specified by a location vector. Multiple receivers may be specified. In the example file in Figure 5.1-4, two receiver locations are specified by a location vector, a threshold, and a radius. The radius is not a radius in a geometrical sense, but indicates the receiver number. Receivers should be numbered from one to the total number of receivers in the file. The receiver number is used in the ray tracing output file to denote which receiver received a given ray. The threshold value is used to accelerate the ray tracing by discarding rays that have a received voltage less than the threshold. This threshold should be used carefully so as not to discard any rays that contain significant energy. The threshold values used to predict the power delay profiles presented in this dissertation were chosen to be below the received power threshold determined from measurements with the same transmitter and receiver locations.

The last two items specified in the ray tracing input file are the TESSELLATION frequency and the FILE_NAME. The tessellation frequency determines the number of rays traced from the source and the ray wavefront size and ray separation. For a tessellation frequency of fifty, over twenty-five thousand rays are traced from the source in threedimensions. The file name indicates the file name of the raw ray tracing output. 


\subsection{Ray Tracing for Propagation Prediction}

\subsubsection{Background}

The program uses "brute force" ray tracing to account for all possible propagation paths from a transmitter to multiple receivers. Although graphical ray tracing programs can take advantage of sending rays in only specified directions, an electromagnetics model must account for all directions relative to both the transmitter and receiver antennas. As computation times increase, ray tracing acceleration techniques are employed to combat the computational requirements of brute force ray tracing [Gla89]. An electromagnetic image theory model, where reflections are modeled as being due to image sources (equivalence), is practical when only a few reflections are to be considered. However, image theory can be cumbersome when a large number of multiple reflections from randomly oriented objects are considered.

The code is written in C++ for use on a UNIX-based workstation. As an objectoriented language, $\mathrm{CH}$ has the capability to manipulate data structures such as vectors, objects, and the functions that match them, in a modular fashion. For example, the program can perform an intersection test for a ray and an object. Although the algorithms to determine the intersection are different for different objects, the same subroutine may be called to perform the intersection test. Due to the numerous ray-object intersection tests and extensive data arrays required for ray tracing, there are considerable requirements on the computer operating platform. The computation and memory requirements make a workstation a minimum requirement.

\subsubsection{Source Ray Directions}

The transmitter and receiver are modeled as point sources in a building. In order to determine all possible rays that may leave the transmitter and arrive at the receiver, it is necessary to consider all possible angles of departure and arrival at the transmitter and receiver. Consider the spherical coordinate system relative to a point source or receiver shown in Figure 5.2-1. Rays are launched from the transmitter at an elevation angle $\theta$ and azimuth angle $\phi$ relative to this fixed coordinate system. 


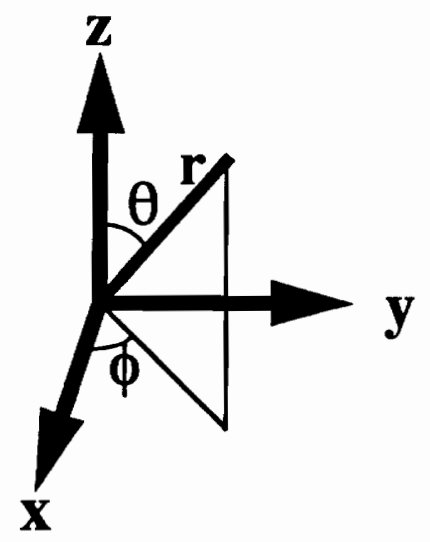

FIGURE 5.2-1. Spherical coordinate system used to reference the ray departure and arrival angles at the transmitter and receiver.

To keep all ray manipulation routines general, it is desirable that each ray tube occupy the same solid angle $d \Omega$, and each wavefront be an identical shape and size at a distance $r$ from the transmitter. An ideal wavefront is shown in Figure 5.2-2. Additionally, these wavefronts must be subdividable so that an increased ray resolution can be handled easily. For reference, let $r=1$ and the total wavefront is the surface of a unit sphere. The problem then becomes one of subdividing the sphere surface into equal area "patches" that are all the same size and shape and completely cover the surface without gaps. For a two-

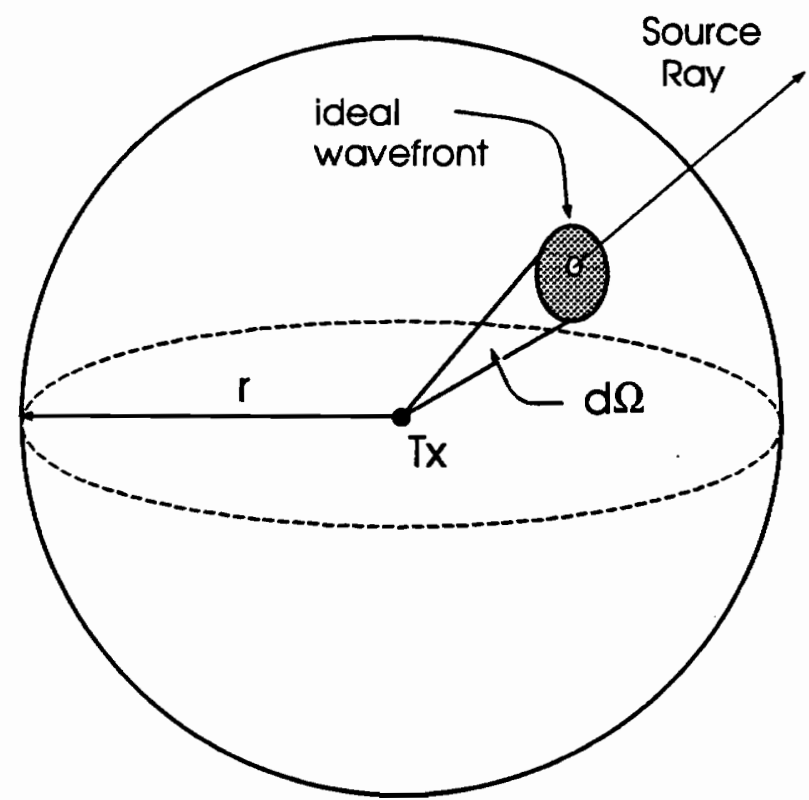

FIGURE 5.2-2. Ideal wavefront represented by each source ray (diagram from [Sch92]. 
dimensional (flat) surface, this problem is easy. Regular triangles, squares, and hexagons can completely cover an area with equal size and shape objects without leaving gaps (see Figure 1.2-1 for hexagons). Constant angular separation of the rays may be easy to visualize, but the mechanics of the problem are rather complicated. Common methods of subdividing the total wavefront, such as by using spherical coordinates, are insufficient. This is due to a decrease in the angular separation between rays as they are launched in directions near the poles of the sphere.

The solution for the source ray directions is adapted from the theory of geodesic domes [Ken76], [Pug76], [Wen79]. An icosahedron is inscribed inside the unit sphere. A regular icosahedron is a twenty sided polygon with twenty triangular faces and twelve vertices. Each vertex joins five faces. Figure 5.2-3a and $b$ show two views of a regular icosahedron. In Figure 5.2-3a, the "top" faces are highlighted, and in Figure 5.2-3b, the

a.)

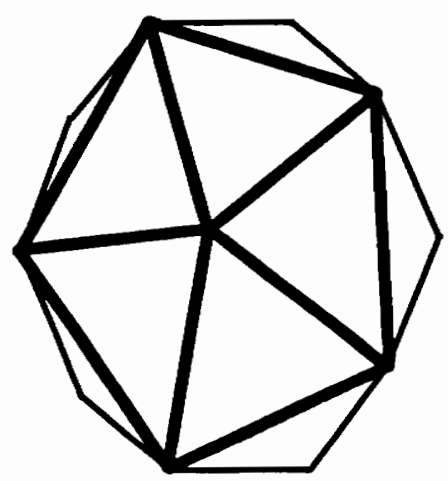

b.)

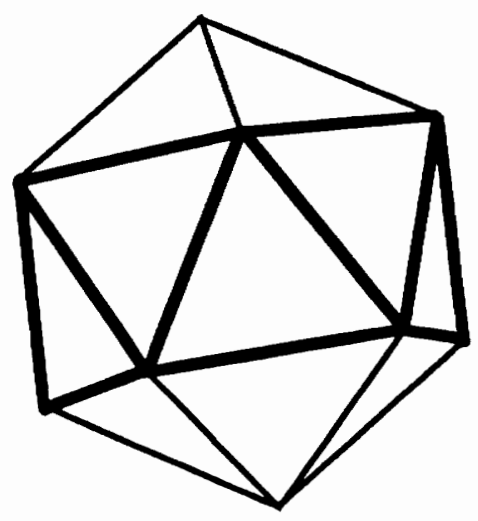

FIGURE 5.2-3. Two views of a regular icosahedron that is inscribed inside a unit sphere. Rays are launched at icosahedron vertices.

"middle" faces are highlighted. If rays are launched at each of the twelve vertices, each ray wavefront is an identically shaped pentagon separated by 63 degrees from each of its five nearest neighbors. A wavefronts's nearest neighbors are the rays whose wavefront "patches" are adjacent to the ray wavefront. 
Since twelve rays with an angular separation of 63 degrees is not enough ray resolution for accurate propagation prediction, each triangular face of the icosahedron is subdivided as follows. Each triangular face is tessellated into $\mathrm{N}$ equal segments where $\mathrm{N}$ is the tessellation frequency [Wen79]. Figure 5.2-4 shows an example with $\mathrm{N}=4$. Lines par-

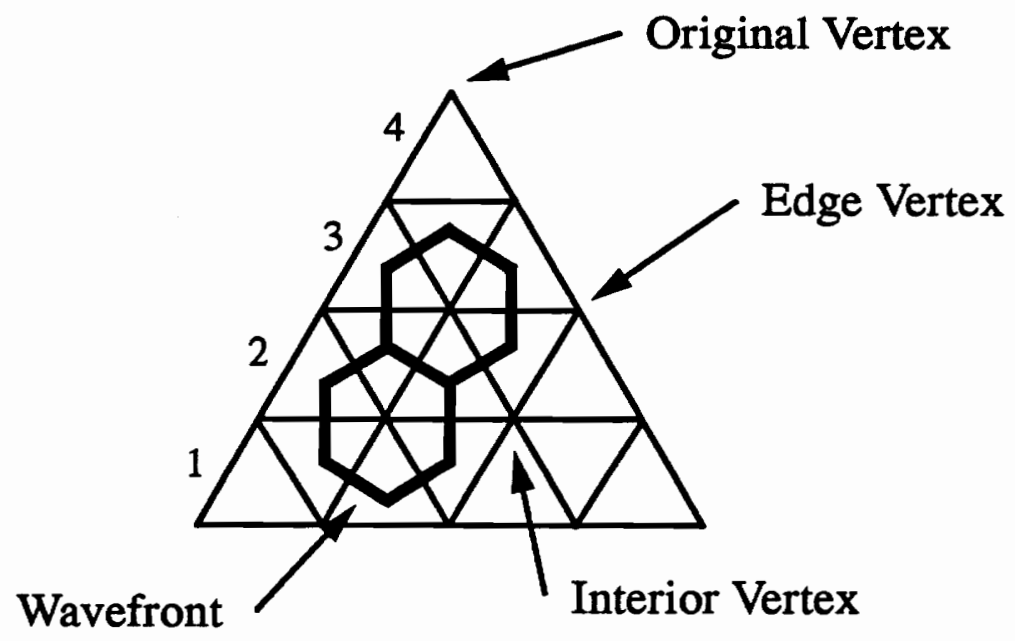

FIGURE 5.2-4. Tessellation of icosahedron face. Ray wavefronts are hexagonal for edge and interior vertices.

allel to one of the three sides are drawn that subdivide the triangle into smaller equilateral triangles. Rays are launched at angles that pass through the vertices of the triangles. Wavefronts are hexagonal for rays that pass through interior and edge vertices. Ray wavefronts that pass through the twelve original icosahedron vertices are pentagonal. Ray wavefronts are hexagonal and pentagonal on the surface of the icosahedron. However, the surface normal of the icosahedron face is not necessarily the ray direction since the icosahedron surface must be projected onto the surface of the unit sphere to determine the true wavefront. This is shown in Figure 5.2-5. As the tessellation frequency increases, the wavefronts decrease in size, but keep their shape and relation to their nearest neighbors. Hexagonal rays have six nearest neighbors, and pentagonal rays have five. The angular separation between a ray and its nearest neighbors is nearly identical for all nearest neighbors. However, the angular separations are slightly different for rays that originate from the three different types of vertices. The number of source rays traced is $10 N^{2}+2$ where $N$ is the tessellation frequency [Wen79]. 


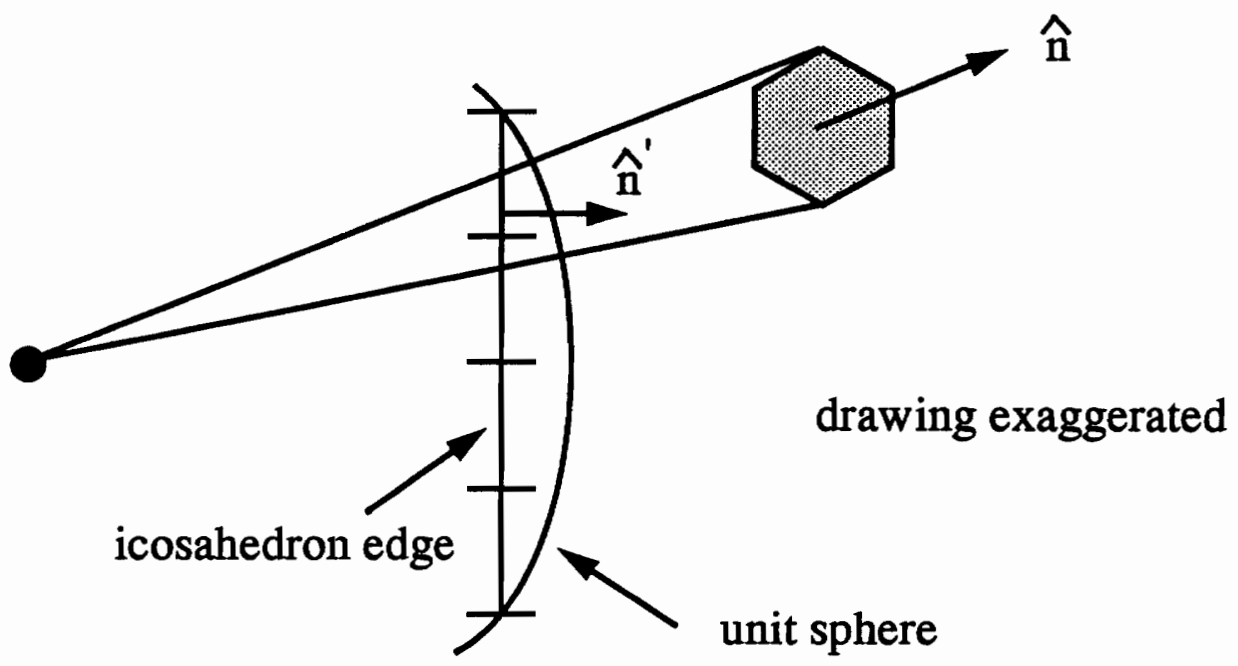

FIGURE 5.2-5. Nearly hexagonal ray wavefront projected onto the surface of the unit sphere.

Figure 5.2-6 shows a plot of minimum and maximum angular ray separation as a function of the number of rays traced. Notice that the angular separation decreases as the number of rays increases. For a maximum angular separation of one degree between rays, 40,000 rays must be traced. This requires a tessellation frequency of 63. In Figure 5.2-7, the solid line indicates the difference in minimum and maximum ray separation between a ray and its neighbor for different source rays. The dotted line is the difference in ray separation for a given source ray and its own nearest neighbors. The curves are slightly different because of the differences between the pentagonal ray wavefronts launched at the original icosahedron vertices and the hexagonal ray wavefronts launched from the tessellated faces of the icosahedron. Thus, this method of launching the source rays provides wavefronts that completely subdivide the surface of the unit sphere with nearly equal shape and area.

\subsubsection{Tracing The Rays at a Boundary}

The computer program uses forward ray tracing to find each ray path by which significant levels of energy radiated from the transmitting location reaches the receiving point. For a given execution of the program, multiple receiving locations can be defined, so the procedure described here can be applied to each receiving point. The ray tracing is 


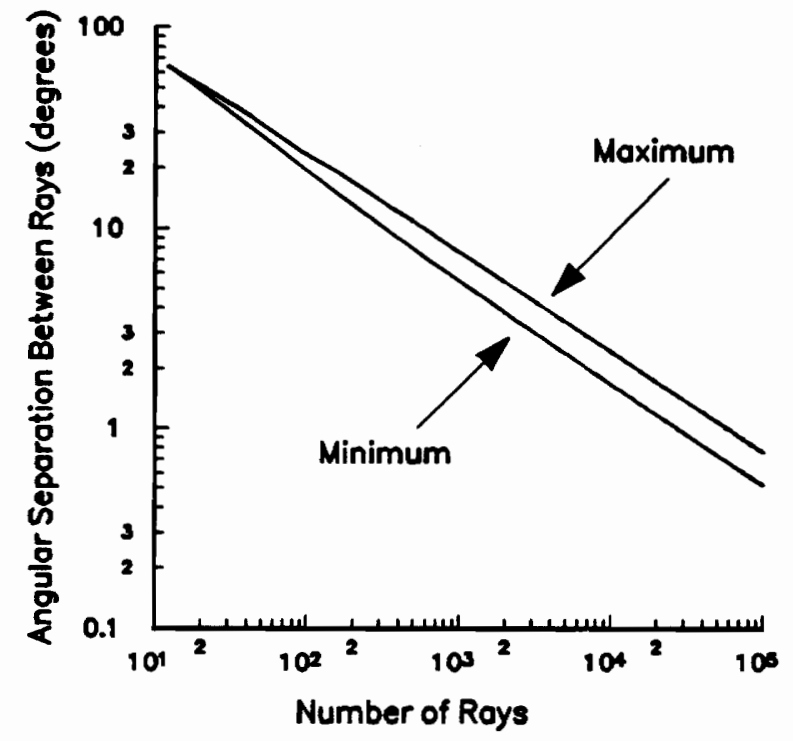

FIGURE 5.2-6. Maximum and minimum ray separation between nearest neighbors as a function of the number of rays traced. The difference between the maximum and minimum is caused by the difference between the pentagonal and hexagonal shaped wavefronts.

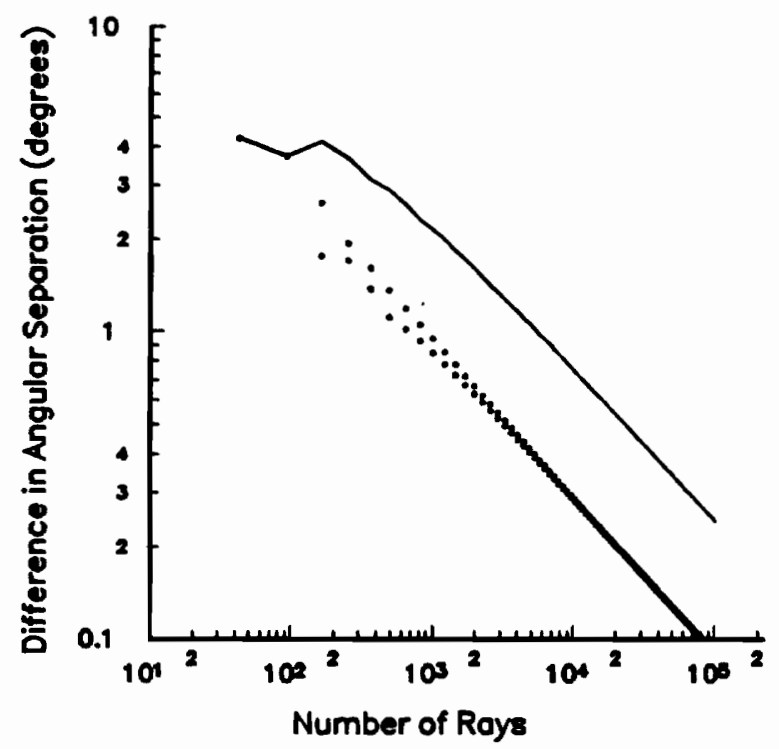

FIGURE 5.2-7. Difference between maximum and minimum ray separation between nearest neighbors as a function of the number of rays traced.

accomplished by an exhaustive search of a ray tree accounting for the decomposition of the ray at each planar intersection. First, the program determines if a line-of-sight path exists, and if so computes the received field. Next, the program traces a source ray in a 
previously determined direction and detects if an object intersection occurs. If no intersection is found, the process stops and a new source ray is initiated. Once the program determines that an intersection has occurred, it then checks to see if a specularly reflected or transmitted ray has an unobstructed path to the receiving location. After checking for reception, the program divides the source ray into a transmitted and reflected ray that are initiated at the intersection point on the boundary. These rays are then treated in a similar fashion to source rays. This recursion continues until a maximum number of tree levels is exceeded, the ray intensity falls below a specified threshold, or no further intersections occur. Figure 5.2-8 shows a portion of a ray tree for one source ray.

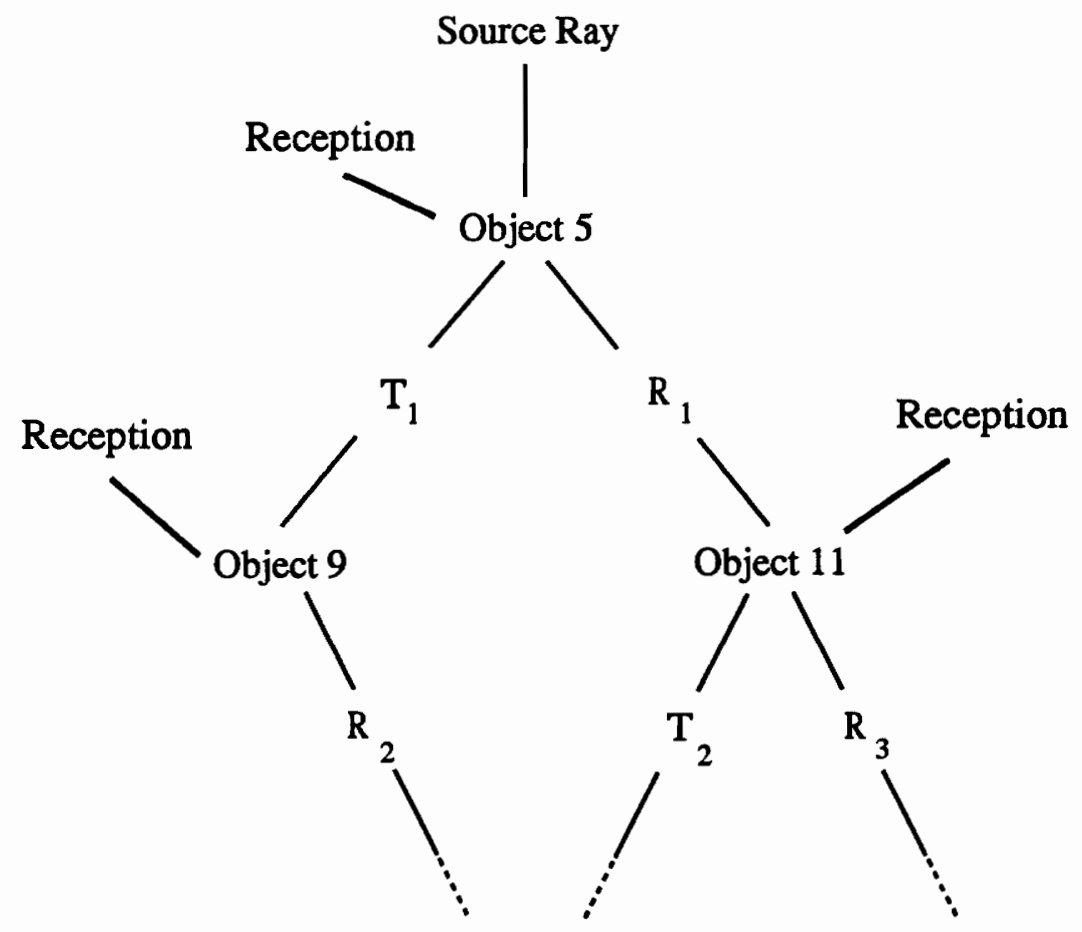

FIGURE 5.2-8. Ray tree that shows how one source ray can be decomposed into many transmitted, reflected, and scattered rays from intersections with planar boundaries.

\subsubsection{Representation of Propagation Models in the Ray Tracing Program}

The propagation of energy from the transmitter to the receiver occurs in various modes such as by direct, reflected, transmitted, and diffracted paths. In considering the 
implementation of each of these in the propagation model, it is important to recognize the path loss dependence of each mode. Direct (line-of-sight) rays exhibit a $1 / \mathrm{d}^{2}$ power dependence according to Friis free space transmission. Specularly reflected and/or transmitted rays follow a $1 / d^{2}$ dependence, where $d$ represents the total ray path length. For example, the specularly reflected ray shown in Figure 5.2-9, whose path segments are labeled $r_{1}$ and $r_{2}$, has a path loss proportional to $1 /\left(r_{1}+r_{2}\right)^{2}$.

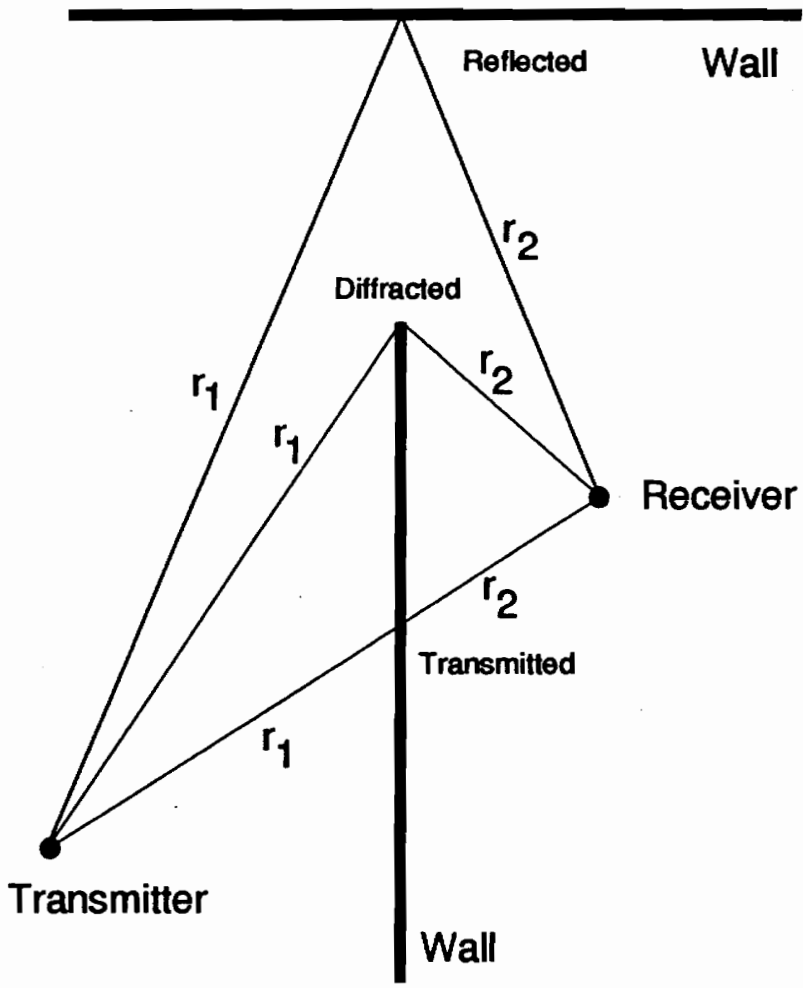

FIGURE 5.2-9. Transmitted, reflected, and diffracted ray paths.

Single diffraction from diffracting corners in a hallway is included where the corner is modeled as a dielectric wedge. The received field is determined from the uniform geometrical theory of diffraction as modified for dielectric wedges. The path loss distance dependence is

$$
L_{i}=\sqrt{\frac{r_{1}}{r_{2}\left(r_{1}+r_{2}\right)}}
$$


where $r_{1}$ and $r_{2}$ are as displayed in Figure 5.2-9. In addition, the diffraction coefficient includes a distance dependence. The uniform geometrical theory of diffraction is described in detail in Section 4.3 Geometrical Theory of Diffraction [Kel62], [Kou74], [Bal89]. The diffraction model accounts for all scattering in non-specular directions. No multiple diffraction or reflection-diffraction is considered in the model.

In summary, the model implements direct, reflected, transmitted, and diffracted fields represented by the rays. Table 5.2-1 summarizes the variables used to describe the model. Each propagation mechanism is treated separately, and the total field is determined via coherent superposition of the individual contributions of each ray as weighted in time by a probing pulse identical to the one used in measurements. This program predicts the channel impulse response (power delay profile) which is a wide band characterization. Wide bandwidth signals have been shown not to vary significantly over small distances due to the inherent frequency diversity in the signal [Gan90], [Gan91], [Te191]. The complex field amplitude of the $i^{\text {th }}$ ray at the receiver is given by

$$
E_{i}=E_{o} f_{t i} f_{r i} L_{i}(d) \prod_{j} \Gamma\left(\theta_{j i}\right) \prod_{k} T\left(\theta_{k i}\right) e^{-j k d}
$$

For a diffracted ray, the complex reflection and transmission coefficients are replaced by the complex diffraction coefficient. 
TABLE 5.2-1: Summary of the variables used to describe the ray tracing propagation model.

\begin{tabular}{|c|c|}
\hline $67 n a b l e$ & Bescription Luntsh \\
\hline $\mathbf{f}_{\mathrm{ti}}$ & Field amplitude radiation pattern of the transmitter antenna \\
\hline $\mathbf{f}_{\mathbf{r i}}$ & Field amplitude radiation pattern of the receiver antenna \\
\hline $\mathrm{L}_{\mathrm{i}}(\mathrm{d})$ & Path loss distance dependence for the $i^{\text {th }}$ multipath component \\
\hline d & Path length [meters] \\
\hline$\Gamma\left(\theta_{i i}\right)$ & Reflection coefficient \\
\hline$T\left(\theta_{k i}\right)$ & Transmission coefficient \\
\hline$E_{i}$ & Field strength of the $i^{\text {th }}$ multipath component $[\mathrm{V} / \mathrm{m}]$ \\
\hline $\mathrm{E}_{0}$ & Reference field strength [V/m] \\
\hline$e^{-j k d}$ & Propagation phase factor due to path length \\
\hline
\end{tabular}

In (5.2-2), the reflection, $\Gamma\left(\theta_{j i}\right)$, and transmission, $\mathrm{T}\left(\theta_{k i}\right)$, losses for the $\mathrm{i}^{\text {th }}$ ray are given as functions of incidence angle, $\theta$. This is to illustrate the dependence of these losses given by the Fresnel formulas. Power delay profiles are computed and path loss is computed by referencing the result of (5.2-2) to a 1-meter free space path loss. The polarization of the wave relative to the interface determines whether the perpendicular or parallel Fresnel reflection coefficients are used. For reflections and transmissions with the floor or ceiling, the parallel coefficients are used, and the perpendicular reflection and transmission coefficients are used when the ray intersects a vertically oriented building wall.

\subsubsection{Identification of Specular Rays}

Consider a transmitter and receiver in the presence of a single planar scattering surface as shown in Figure 5.2-10. To determine the ray traced impulse response by a brute force method, it is necessary that the solution include only one specular ray regardless of how many rays are traced. The test to determine if the ray reaches a receiving point is accomplished in the following fashion. A perpendicular projection from the receiving location to the ray path is computed and the total (unfolded) path length, $d$, that the ray travels from the transmitter to the projection point is determined. A reception sphere (from [Hon91], extended to three dimensions [Sch92], [Sei92b]) is constructed about the receiv- 


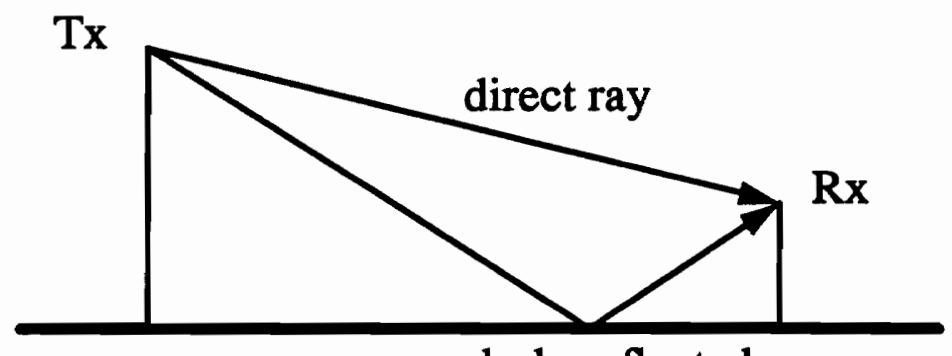

specularly reflected ray

FIGURE 5.2-10. Transmitter and receiver in the presence of a specularly reflecting surface. A ray tracing solution must include only one specularly reflected ray for a given surface.

ing location with a radius proportional to the unfolded path length and the angular spacing between neighboring rays at the source. If the ray intersects the reception sphere, it is received and contributes to the total received signal. Otherwise, the ray is not received. Regardless of whether or not the ray is received, recursion proceeds as previously described. The reception sphere effectively accounts for the divergence of the rays from the source. For ray separation $\alpha$ sufficiently small, the ray intercepting the sphere will be an accurate measure of the ray that would pass directly through the receiving point. The physical interpretation of the reception sphere can be justified with the aid of Figure 5.211. This figure is a two-dimensional representation of a ray being traced. Two adjacent rays, launched at $\pm \alpha$ relative to the test ray, are also shown. Note that in three dimensions, any ray will have more than two adjacent rays and the angular separation of the adjacent rays will not necessarily coincide with the coordinate axes. As shown in the figure, a reception sphere with the correct radius can receive only one of the rays. If the radius is greater than $\alpha \mathrm{d} / \sqrt{3}$, two of the rays could be received and would, in effect, count the same specular ray path twice. Likewise, if the radius is too small, none of the rays will intercept the sphere and the specular energy will be excluded. It is important to emphasize that the reception sphere radius is proportional to the unfolded path length from the source to receiver and is different for each ray path.

Recall Figure 5.2-4 where $\alpha$ is the angular separation between rays at the source. In order to make sure that the specular point is not missed, the ray wavefront is considered 


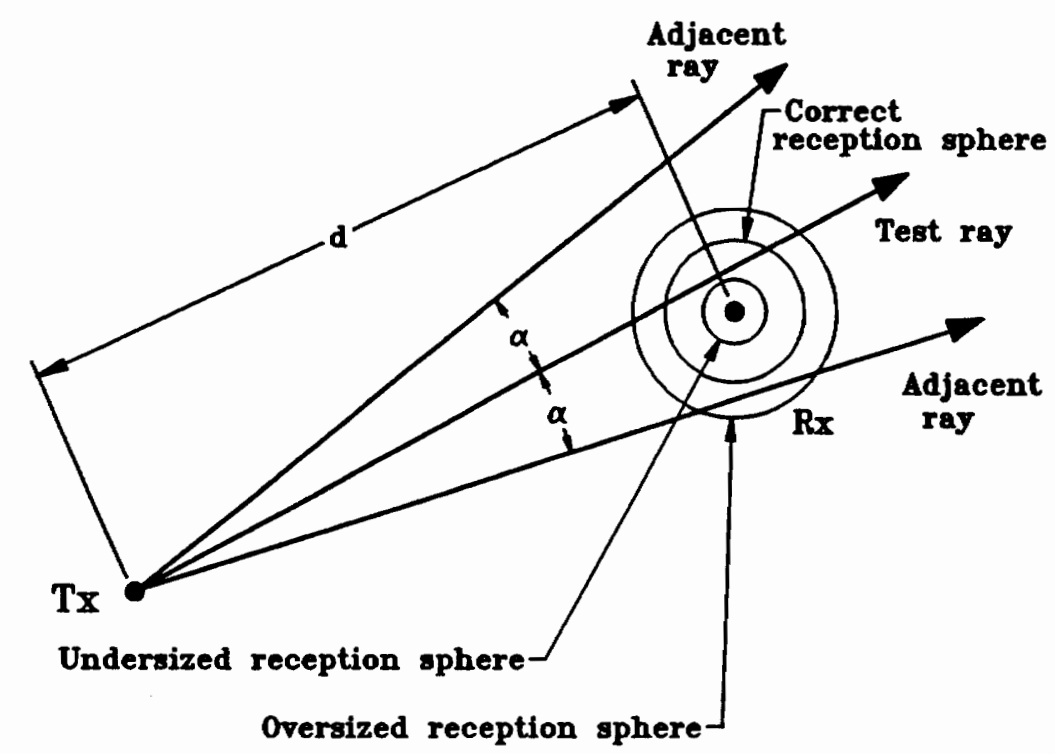

FIGURE 5.2-11. Two dimensional view of the reception sphere. The total ray path length is $d$ producing a reception sphere radius of $\alpha d / \sqrt{3}$ (from [Sch92]).

as a circle circumscribed about the hexagonal (or pentagonal) wavefront shape. Figure 5.2-12 shows two adjacent rays and their respective wavefronts. The angular radius of the circumscribed circle is $\alpha / \sqrt{3}$. Although it is possible to receive two specular points with this approach, the path loss error of such a case would be $3 \mathrm{~dB}$. A missed specular point could lead to a much larger error if a significant amount of energy is carried by that ray. Adjacent rays that are received by overlapping reception spheres are eliminated during the processing of the raw ray tracing output. The use of a reception sphere requires that the rays must be launched such that each ray is separated from neighboring rays by a nearly constant angle, $\alpha$. If nearly uniform separation is not maintained, the test ray will not be

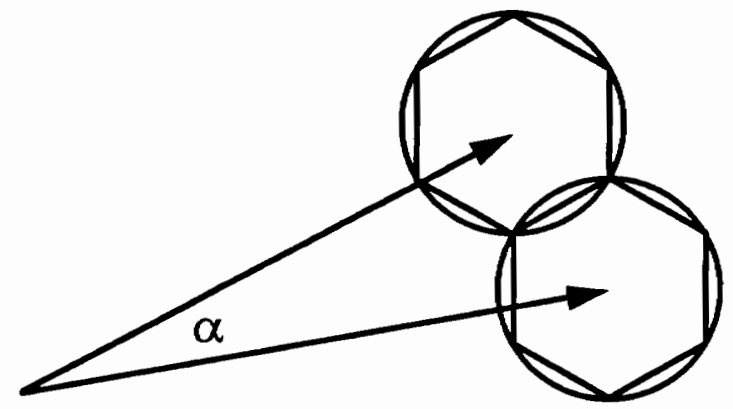

FIGURE 5.2-12. Illustration of assumed wavefront for the purposes of the reception sphere. There is slight overlap between adjacent rays. 
separated from adjacent rays by similar angles and the reception sphere loses its physical significance. All rays that are received are written to a raw ray tracing output file. The format of the output file is described in detail in Section 9.4.1 Raw Ray Tracing Output File (.RAW).

The reception sphere provides a method for identifying specular components. The received specular component indicates the source ray wavefront that most closely approximates the true wavefront. The accuracy of this method is directly related to the size of the reception sphere. The size of the reception sphere is determined by the separation between source rays and the distance travelled by the received ray. The propagation distance is fixed, but the tessellation frequency can be changed to directly affect the error introduced by using a brute force ray tracing method that incorporates a reception sphere. It may be possible that a bound on the maximum reception sphere size could be used to determine the minimum tessellation frequency for a specified error introduced by the ray tracing method. This error is not the error caused by the difference between the measured and predicted propagation, but it is the error introduced by the implementation of the propagation model.

\subsection{Diffraction}

The implementation of diffraction in the computer program is separate from the brute force ray tracing. Since there is no recursion, the diffracted ray paths may be found by a straightforward search for all paths that satisfy the correct geometry for a diffracted ray. Diffracting corners are modeled as dielectric wedges. Diffracted rays are found for all combinations of transmitter and receiver that have a direct path to the diffracting wedge. The locations of diffracting wedges are explicitly specified in the building database and the ray file. The amplitude and phase of diffracted rays are determined by the uniform geometrical theory of diffraction for a dielectric wedge as described in Section 4.3 Geometrical Theory of Diffraction [Kel62], [Kou74], [Bal89]. 


\subsection{Processing Raw Ray Tracing Program Output}

The raw ray tracing output is stored in a $R A W$ file. The raw ray tracing output undergoes several stages of data processing before power delay profiles are generated. First, the original $R A W$ file is separated into a $R A W$ file for each receiver location. The multipath components in each.$R A W$ file are then sorted by excess delay and stored in a sort file for each receiver location. Once the individual impulses are sorted, they can be convolved with a probing pulse to determine power delay profiles. This section describes the format of these different ray tracing output files.

\subsubsection{Raw Ray Tracing Output File (.RAW)}

The first step in processing the raw output from the ray tracing program $(R A W$ file) is to separate the data by receiver location. The raw output file contains the individual multipath components for all of the receiver locations specified in the ray file. A $R A W$ file is separated into a $R A W$ file for each receiver location. Figure 5.4-1 gives a sample of a $R A W$ file that contains raw ray tracing output.

The information for each individual multipath component is given on three separate lines. The first line contains the component number, receiver number, ray type, number of levels, propagation distance, received field strength, and ray departure angles at the transmitter and ray arrival angles at the receiver. The two strings on the second line contain the reflection and transmission history of the ray, and the third line contains the incidence angle history of the ray. These values are described in more detail in the following paragraphs.

The component number is an artifact of the ray tracing and has no significance in processing the data. The receiver number is used to separate the original $R A W$ file into a $R A W$ file for each receiver. The ray type can take on acceptable values of $1,100,200$, and 1000. A ray type of 1 indicates a line-of-sight ray. Ray types of 100 or 200 indicate a specularly reflected or transmitted ray, respectively. The ray type pertains to the last reflection or transmission before being received. Diffracted rays are assigned a ray type of 1000 . The number of levels is the number of ray tree levels the ray traversed before being received. 


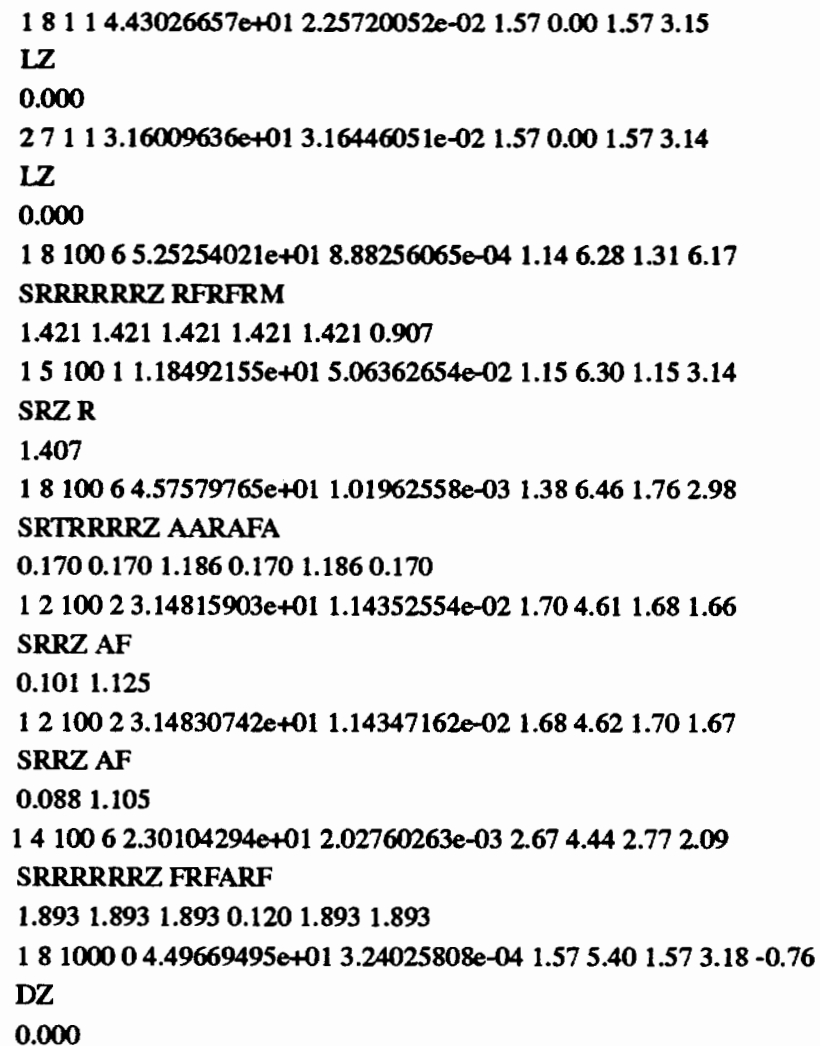

FIGURE 5.4-1. Sample ray tracing output file (.RAW).

This number is the number of reflections or transmissions the ray experiences between the transmitter and receiver for ray types 100 or 200 . The next number is the propagation distance in meters from the transmitter to the receiver. The propagation delay is proportional to the propagation distance. The received field strength relative to the field strength at one meter over a free space path is the next item in the file. This number represents the path loss distance dependence without consideration of the reflection or transmission coefficient. The last four numbers on the first line represent the ray departure and arrival angles at the transmitter and receiver. All angles are measured in radians using standard spherical coordinates shown in Figure 5.2-1. The first two numbers are the departure elevation (theta) and azimuth (phi) angles, respectively, and the last two numbers are the arrival elevation and azimuth angles. The ray departure and arrival angles are included so that the elevation and azimuth antenna patterns at the transmitter and receiver can be included in 
the model. For diffracted rays, an additional number on the line represents the phase shift in radians caused by the diffracting corner.

The second line in the $R A W$ file for an individual multipath component is a ray history of the received component. The first string is the reflection and transmission history, and the second string is the material interaction history. The first character in the first string will be an $\mathrm{L}$, an $\mathrm{S}$, or a $\mathrm{D}$ for line-of-sight, specularly received rays, and diffracted rays, respectively. The last character in the first string will be either a $Z$ or an $X$. The letter $Z$ indicates that the ray was received with no resolution problems due to the tessellation frequency. If the string is terminated by the letter $\mathrm{X}$, this indicates that for this individual multipath component, there was not enough ray separation resolution to determine if the component should be considered specular. In between the first and last characters are a combination of the letters $R$ and $T$. The letter $R$ indicates a Reflection, and the letter $T$ indicates a Transmission. The second string has a character for each $T$ or $R$ in the first string. Each character represents the name of the material that the ray reflected off of or transmitted through. Recall that the name of the material was specified in the ray file for each object. Materials are classified by their electrical properties. There is no second string for line-of-sight or diffracted components since these components do not undergo any transmissions or reflections. The information in these two strings facilitates investigation of the ray history of each individual multipath component.

The third line of the file for each multipath component is a history of the incidence angles for each reflection or transmission. There is one number in the file for each reflection or transmission. The number is the negative of the dot product of the incidence angle and the surface normal. The negative sign is simply a convention used to determine the positive angle between the incident ray and the surface normal. The value of the dot product ranges between 0 and 1 . In order to incorporate some polarization dependence, interactions with the floor and ceiling have the value of 1 added to them. In this way, it is possible for the polarization to be distinguished from the number in the file that ranges between 0 and 2 . If the value is between 0 and 1 (inclusive), the value is the dot product of a horizontally (TE) polarized interaction, and if the value is between 1 (exclusive) and 2 , the value 
is one plus the dot product of a vertically (TM) polarized interaction. Line-of-sight and diffracted components have the incidence angles set to zero, and these values are not used in processing these types of components.

\subsubsection{Sorted Ray Tracing Impulses (.sort)}

Once the data is separated by receiver location, a sort file is created which contains the received multipath components sorted by increasing excess delay. The sort files are created and multiple representations of identical multipath components that may have been captured by overlapping reception spheres are eliminated. Part of a sort file can be seen in Figure 5.4-2. The first line contains the ray number, the receiver number, the ray type, the excess delay in nanoseconds, the received field strength relative to the value measured at one meter over free space, the departure and arrival angles, and the two ray reflection history strings. The second line contains the ray incidence angle history.

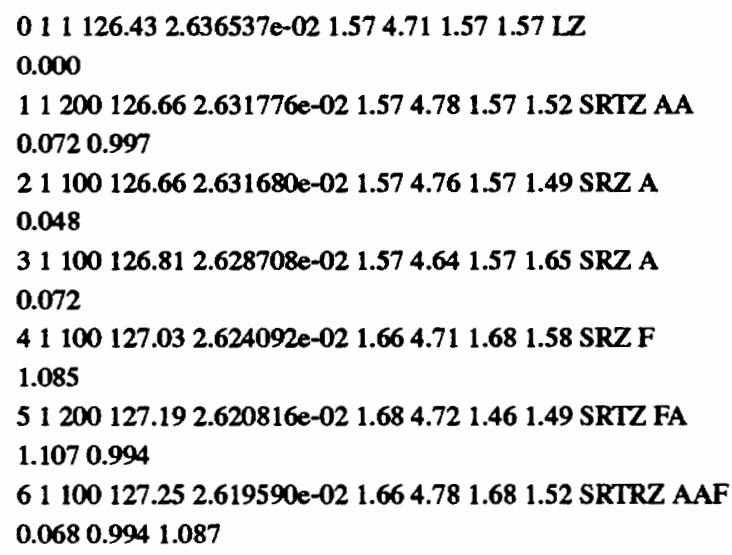

FIGURE 5.4-2. Sample ray tracing sorted impulse file (.sort).

\subsubsection{Power Delay Profiles}

After the raw data is sorted, each multipath component contained in the .sort file is convolved with a probing pulse and combined into a power delay profile that represents the received power as a function of propagation time delay to a resolution determined by the width of the pulse. Each multipath component amplitude is multiplied by the appropri- 
ate reflection, transmission, and diffraction coefficients. Each component amplitude is also multiplied by a digitized representation of the measured antenna elevation pattern. Thus, this representation is the complete predicted power delay profile that includes the amplitude and phase effects of the propagation distance, antenna pattern, and the reflection, transmission, and diffraction coefficients. An example of a few lines of a power delay profile is given in Figure 5.4-3. The first column is the total propagation delay in nanoseconds, and the second column is the path loss in decibels relative to the path loss along a one meter free space path. A simple $x-y$ plot of this file is the power delay profile. When the power delay profiles are optimized for different material properties, these power delay profiles are stored on disk with the extension.$p d p$. A ray tracing power delay profile appears as in Figure 3.3-1.

115.000000200 .000000 116.000000200 .000000 117.000000200 .000000 118.00000067 .866926 119.00000064 .276416 120.00000060 .094472 121.00000051 .410642 122.00000041 .566983 123.00000033 .825538 124.00000028 .791239 125.00000026 .152324 126.00000025 .654577 127.00000025 .822149 128.00000025 .267775 129.00000024 .555686 130.00000027 .153538 131.00000030 .113113 132.00000033 .130977 133.00000035 .437432 134.00000034 .576787 135.00000034 .020623 136.00000034 .850227 137.00000036 .481527 138.00000039 .427796

FIGURE 5.4-3. Sample ray tracing power delay profile file (.pdp file). 


\subsection{Summary}

This chapter has described the primary contribution of this dissertation, a ray tracing propagation model to predict power delay profiles for a transmitter and receivers located on the same floor inside a building. The computer representation of the building in AutoCAD and the conversion to a standard format have been described. Direct, reflected, transmitted, and diffracted rays have been implemented into the program. A novel method for determining the source ray directions in three dimensions based on the theory of geodesic domes was developed. A ray tree was described to show how the program implements recursion of multiply reflected and transmitted rays. A reception sphere in three dimensions is used to determine when rays may be considered specular. The conversion of the raw ray tracing output to power delay profiles and the various file formats have been described. 


\section{Comparison of Measured and Predicted Propagation}

\subsection{Overview}

The validation of any propagation prediction model must include comparison with measured data. For ray tracing methods that relate the propagation to specific objects in the physical radio channel, this comparison must include both multipath component amplitudes and arrival times in order to quantify the accuracy. This comparison must also be on a specific location-by-location basis. The measured data presented here are power delay profile impulse response estimates that contain no phase information. The resolution is determined by the pulse width of the measurement system. Power delay profiles are computed from the predicted individual multipath components in order to compare measured and predicted propagation data. Two different methods are used here to compare the measured and predicted propagation data. First, measured and predicted power delay profiles are compared on a location-by-location basis as a function of excess delay. An error function is defined to quantify the 'distance' between the two curves. The second method of comparison is a location-by-location comparison of propagation characteristics such as path loss, COST 207 delay interval, and rms delay spread that are computed from the power delay profiles. Both of these methods compare measured and predicted propagation data at specific locations in a manner that incorporates both the amplitudes and arrival times of individual multipath components. This location-by-location comparison of measured and predicted propagation is a significant contribution of this dissertation since previous work in ray tracing prediction methods in buildings have not been validated by such a comparison.

Previous ray tracing models have not compared measured and predicted propagation data in terms of both the amplitudes and arrival times of individual multipath components in a site-specific manner. In [McK91] and [Dri92], ray tracing was used to predict coverage of a radio system in a building, but no measured data were available for comparison. Comparison of average signal strength (path loss) as in [Hon92] is a necessary measure of the model accuracy, but is not sufficient to determine the accuracy of the model since there are many possible combinations that can predict the same average signal 
strength as the measured data. However, only narrow band signal strength measurements were available for comparison in [Hon92], and the work contained therein is the first to verify a ray tracing model for propagation prediction with measured data. Similarly, the multipath component arrival times must be compared for specific locations of measurements and predictions. In [Law92], the cumulative distribution functions of measured and predicted rms delay spreads were computed for a particular set of rooms inside a building. However, the relationship between the individual measured and predicted rms delay spreads was not determined, and the power delay profiles at individual locations were not compared. In addition, rms delay spread is not necessarily a good measure of the prediction accuracy since two quite different power delay profiles can have the same rms delay spread. Hence, a comparison criteria that incorporates the multipath component arrival times in a power delay profile is required to determine the accuracy of a ray tracing propagation prediction model. Here, a location-by-location power delay profile comparison, and a path loss and delay interval comparison are used to quantify the prediction accuracy. Rms delay spread results are also presented since this is a more common measure of the time dispersion of a radio channel.

Because the exact building material properties are unknown, a method for minimizing the mean square error between measured and predicted power delay profiles as a function of excess delay is developed. The predicted power delay profiles are modified by implementing different amplitude and phase models for the reflection and transmission coefficients. The reflection and transmission coefficients are varied by changing the dielectric properties of the building materials used for prediction. The reflection and transmission coefficient models that give the minimum mean square error over an ensemble of measurement locations are used to determine the predicted power delay profiles presented here.

Next, measured and predicted power delay profiles are compared at $4.0 \mathrm{GHz}$ for two buildings at $1.3 \mathrm{GHz}$ in one of the buildings. This provides a qualitative comparison between measured and predicted power delay profiles for a variety of measurement locations with different surroundings. Finally, a location-by-location comparison of the propa- 
gation parameters determined from measured and predicted power delay profiles is given. The standard deviation of path loss error is less than five decibels for all measurement locations, and this error is achieved over a total path loss dynamic range of fifty decibels. It is shown that a $16 \mathrm{~dB}$ change in measured path loss as a receiver is moved from a lineof-sight to an obstructed location is predicted for the same locations. Although there are several locations where the measured and predicted values disagree, comparison of delay interval and rms delay spread show good agreement between measured and predicted propagation parameters in many cases.

\subsection{Comparison Criteria and Error Function Definition}

Consider a measured and a predicted power delay profile as shown in Figure 6.1-1. Some measure of the 'distance' between the two curves is desirable. A quantitative measure of the error that incorporates both the amplitudes and arrival times of the individual multipath components is a useful measure of the 'distance' between measured and predicted power delay profiles. The measure used is the mean square error between the two curves on a pointwise basis at one nanosecond intervals. The error between the two curves on a sample-by-sample basis is computed for all samples that have an amplitude above the received power threshold for either the measured or predicted power delay profile. These values are computed as the difference in decibels. Samples that are below the threshold for both measured and predicted profiles are not included in the calculation. When the sample at a constant time delay is above the threshold for one profile and below the threshold for the other, the error is considered to be the difference between the amplitude above the threshold and the threshold value. This prevents large errors due to missed multipath components from significantly weighting the calculation. For each comparison of measured and predicted power delay profiles, an error curve that is the difference between the measured and predicted power delay profiles as a function of excess delay is computed. The optimization is to minimize the total area under all of the squared error curves. Figure 6.11 shows measured and predicted power delay profiles and the error curve as a function of excess delay for a typical measurement location on the second floor of Whittemore Hall. Note that the error curve is displayed in the figure and the area under the squared error 
curve is minimized to determine the reflection and transmission coefficient models. The error curve ranges between plus and minus ten decibels. Hence, at some excess delays, the predicted power is too large, and at other excess delays, the predicted power is too small.
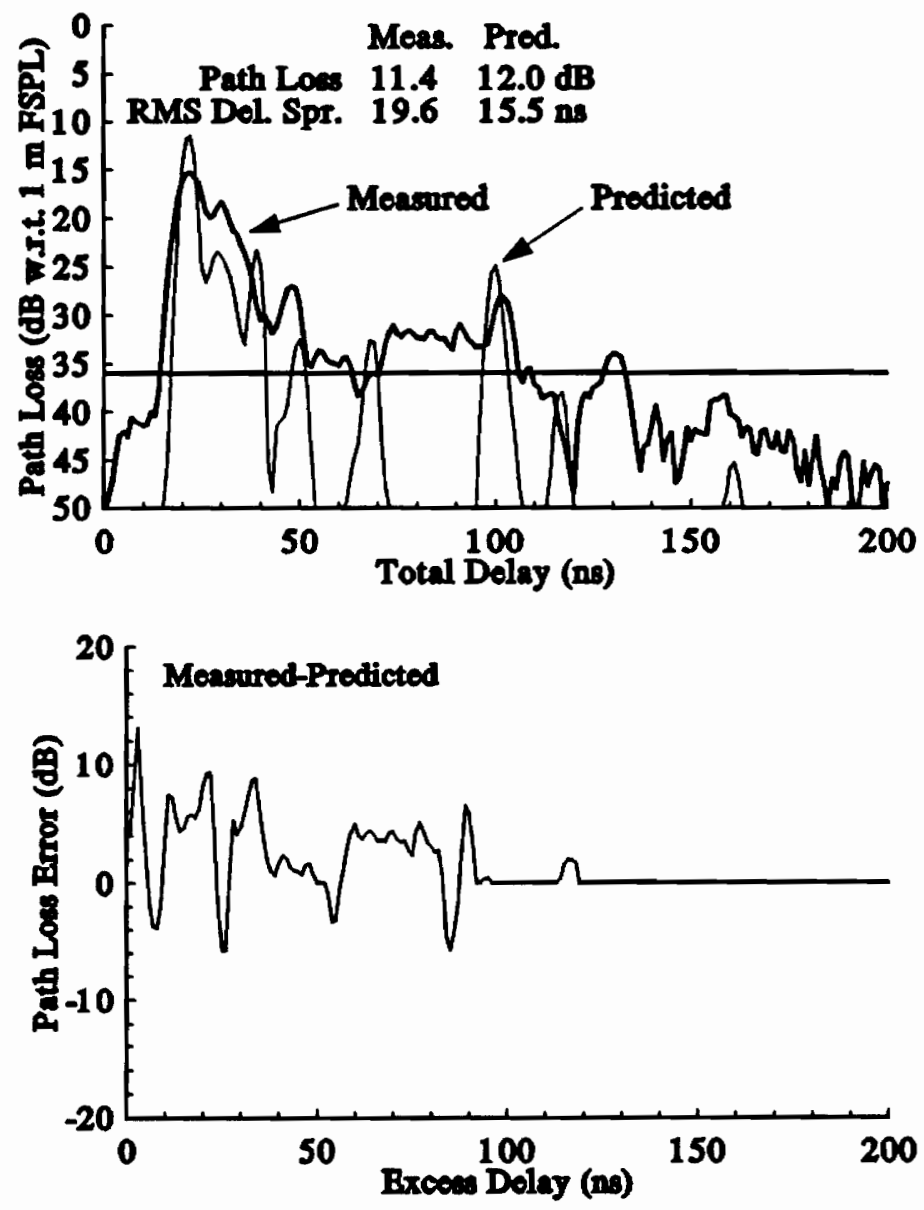

FIGURE 6.1-1. Measured and predicted power delay profiles and the error curve as a function of excess delay measured on the second floor of Whittemore Hall on the Virginia Tech campus.

This method assumes that the arrival times of measured and predicted multipath components are aligned. If a measured and predicted multipath component have the same amplitude, but are offset in time delay by a small amount, the error between the two components computed by this method would contribute to the overall error. However, this case could indicate that the error in the predicted multipath component is only in time delay and not in the predicted amplitude. This point is mentioned to suggest that future methods 
of determining the 'distance' between measured and predicted power delay profiles consider the possibility of arrival time as well as amplitude differences of individual multipath components.

The difference between the measured and predicted power delay profiles as a function of excess delay is computed for each combination of transmitter and receiver. Two different optimizations are performed. The first optimization is computed for each individual location. The second optimization considers all locations at the same frequency in the same building. These two optimizations are used to determine the penalty for using the results from a combination of locations as compared to the smallest possible error determined by optimizing for each individual location.

\subsection{Optimization of Effective Building Material Properties}

\subsubsection{Optimization Method}

Because the exact building material properties are often unknown, a method for minimizing the mean square error between measured and predicted power delay profiles as a function of excess delay is developed. The ray tracing program determines the arrival times and path loss distance dependence of each received multipath component from the site-specific building database. The only variable that can be used to change the predicted power delay profile is the building material properties. The predicted power delay profiles are modified by implementing different amplitude and phase models for the reflection and transmission coefficients. The reflection and transmission coefficient models that give the minimum mean square error for all excess delay are then used to determine the predicted power delay profiles for forty-five locations in two different buildings. The mean square error function is used for optimization since this representation incorporates the most information about the amplitudes and time delays of individual multipath components. The channel parameters such as path loss and rms delay spread are not used since the measured and predicted values of these parameters could be quite close even though there are major differences in the power delay profiles. 
Each wall is modeled as a homogeneous infinitely thin flat planar surface infinite in extent with a constant relative dielectric constant and conductivity. In addition, all walls in a building with the same name specified in the ray file are considered to be identical. Ideally, the material properties of each individual wall represented in the database would be known in advance. However, the dielectric properties of many common building materials are as yet, unknown. Also, the building walls are rarely (if ever) homogeneous, and are never infinite in extent or infinitely thin. Since the material properties are unknown, an alternate method is used to determine the appropriate material properties that should be used in a particular building to predict the power delay profiles. The ray tracing propagation model uses the Fresnel plane wave reflection and transmission coefficients to determine the amplitudes of individual multipath components. Hence, the approach is to determine the effective material properties for the walls in the buildings under consideration. These effective material properties are the material properties that, when put into the Fresnel reflection and transmission coefficient equations, give the equivalent amplitude (in a minimum mean square error sense) of the reflections and transmissions measured in the real-world, finite-size, non-homogeneous environment. Thus, the building material properties are optimized to minimize the area under the mean square error curve.

Two different effective material properties are computed for each measurement (and prediction) location. First, the effective material properties are computed solely as a function of each measurement/prediction location. That is, only the samples for the measured and predicted power delay profiles from one location are used to optimize the effective materials based on the mean square error. These effective material properties are computed for each combination of transmitter and receiver location in a building. Optimization of the error for each individual location indicates the smallest possible error that can be obtained when only the material properties (reflection and transmission coefficients) are varied over an appropriate range. The effective material properties are also computed for an ensemble of measurement locations at the same frequency in the same building. The mean square error is minimized for several different combinations of transmitter and receiver location at once. This provides overall 'best-fit' values of the effective 
material properties for the particular ensemble when all walls of the same type are considered identical and homogeneous.

\subsubsection{Optimization Results}

Optimization was performed for three different sets of measured data. Measurements were made in Whittemore Hall at $1.3 \mathrm{GHz}$ and $4.0 \mathrm{GHz}$, and in Norris Hall at 4.0 GHz. Both buildings are on the Virginia Tech campus and the measurements are described in detail in Section 6.3 Comparison of Measured and Predicted Power Delay Profiles. Whittemore Hall is an office building with offices and classrooms that are located along a central hallway that snakes throughout the building on the second floor. Most walls are made of drywall mounted on metal studs. These walls separate individual offices from one another and the hallway. The walls that surround the stairwells at each end of the building are cinderblock and poured concrete. For the purposes of building material optimization, all walls are considered identical. Norris Hall is an older building and is constructed out of cinderblock. Offices are located along an L-shaped hallway. At the end of one hallway, there is a large metal door to a laboratory. This door is modeled as a perfect electric conductor with a reflection coefficient of -1 regardless of incidence angle. All of the cinderblock walls are modeled as a homogeneous dielectric.

The optimization was performed over a range of relative permittivities of 1 to 10.8 . A loss tangent $\left(\sigma / \omega \varepsilon_{0}\right)$ of $15 \times 10^{-4}$ was included to incorporate a slight loss for each wall. In Whittemore Hall, the dielectric constant that minimizes the squared error functions is $\varepsilon_{\mathrm{r}}=4.4$ at both $1.3 \mathrm{GHz}$ and $4.0 \mathrm{GHz}$. These values were determined independently. In Norris Hall, the best-fit effective dielectric constant is $\varepsilon_{\mathrm{r}}=7.4$. The resultant Fresnel reflection and transmission coefficients as a function of incidence angle are shown in Figure 6.2-1. These effective material properties were determined for each specific combination of measurement locations in the two buildings at the two frequencies. The resultant values of $\varepsilon_{\mathrm{r}}=4.4$ and 7.4 fall between the relative dielectric constant values of 2.4 for plicene cement and 8.8 for marble [Von54]. The values of the material properties of a concrete wall were derived from propagation measurements at $1.2 \mathrm{GHz}$ in [Hor86]. The reflection coefficients were found to vary between 0.33 and 0.4 , and the relative dielectric constant 
was between 3.9 and 5.4. Hence, the values determined here for the effective relative dielectric constant seem reasonable. The Fresnel reflection and transmission coefficients are not sensitive to small changes in the dielectric constant so that this ray tracing method may be used with confidence even when the optimal effective building materials are unknown.
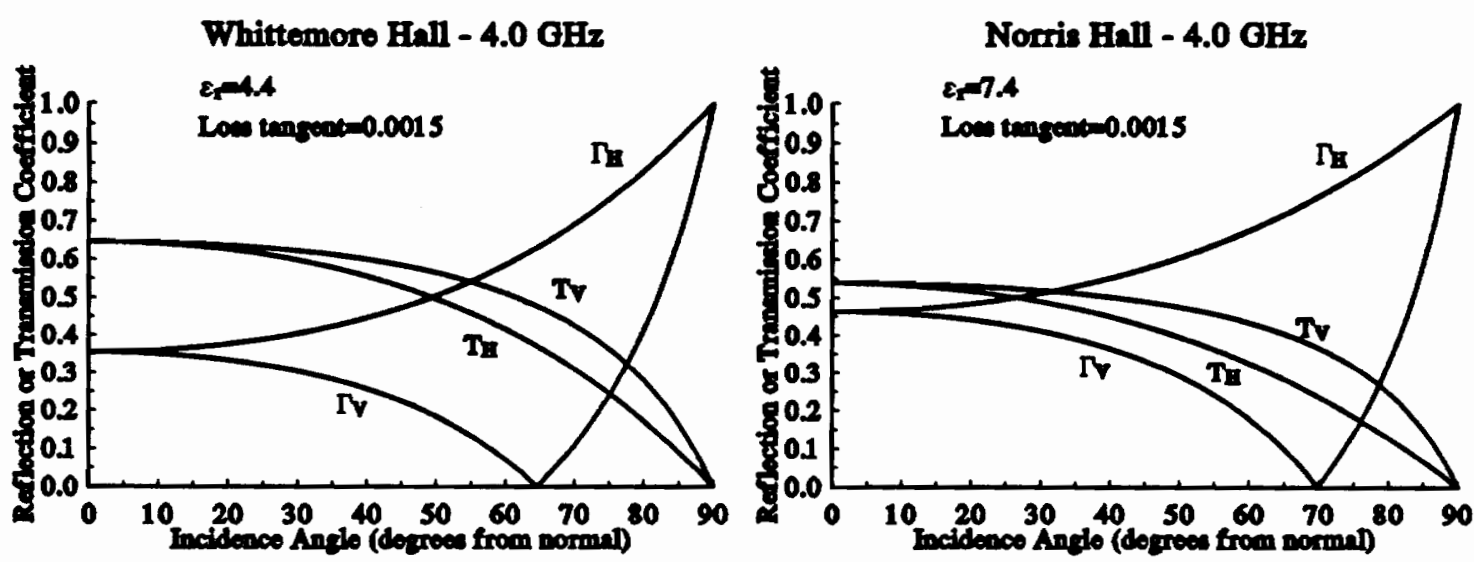

FIGURE 6.2-1. Magnitude of the Fresnel transmission and reflection coefficients at $4.0 \mathrm{GHz}$ for the effective building material properties of $\varepsilon_{\mathrm{r}}=4.4$ in Whittemore and $\varepsilon_{\mathrm{r}}=\mathbf{7 . 4}$ in Norris.

\subsubsection{Analysis of Optimized Error Function With Excess Delay}

In order to determine if there are any excess delays where the prediction error is consistently positive, negative, or large, the average and square root of the mean square error as a function of excess delay is determined. The error functions from all measured and predicted locations are used to compute the statistics. The building and frequency dependence is incorporated in the prediction for each location. Only non-zero errors were included in the calculation since a zero error indicates that both the measured and predicted power delay profiles are below the threshold. The average and square root of mean square error functions are shown in Figure 6.2-2. The average error is shown in the top figure, and the square root of the minimum mean square error is shown in the bottom figure. The error statistics are shown for optimization based on each location and for optimization for the ensemble of locations. Notice that the average error is close to zero for all excess delays with a maximum of about $4 \mathrm{~dB}$. This indicates that the optimization process has provided a near zero-mean error function and does not consistently predict too much or 
too little power at particular excess delays. The square root of mean square error, however, is larger for excess delays less than fifty nanoseconds. This indicates that a majority of the error is due to incorrect calculations of the first-arriving multipath components. Much of this error can likely be attributed to obstructed locations where the first few paths pass through rooms that contain furniture and thick rather than infinitely thin walls. These unmodeled objects may attenuate the measured signal leading to prediction error. From the curves in Figure 6.2-2, it can be seen that there is little difference between the individual and combination optimization errors. Hence, the best-fit building material properties do not vary significantly from location to location, and the effective material properties model gives close to the smallest possible error for the multipath components predicted by the ray tracing program.

Average Ror

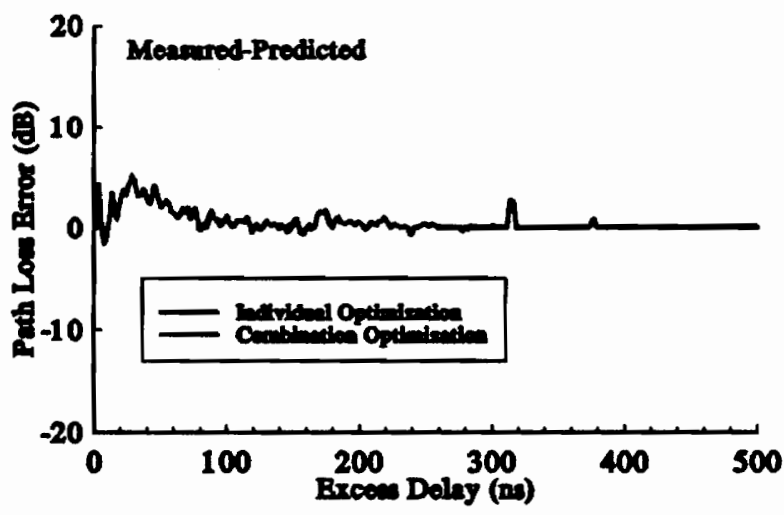

Square Root of Mean Square Error

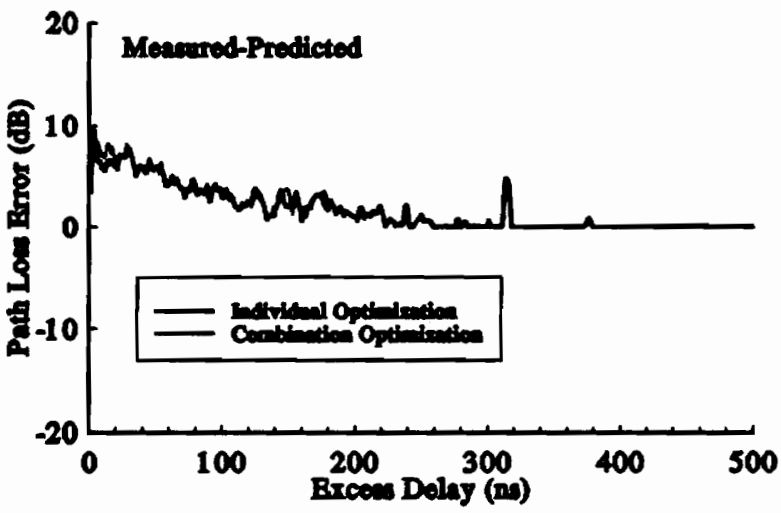

FIGURE 6.2-2. Average and square root of the mean square error function of the difference between measured and predicted power delay profiles as a function of excess delay. 


\subsection{Comparison of Measured and Predicted Power Delay Profiles}

This section provides a qualitative comparison of measured and predicted power delay profiles. The measured and predicted power delay profiles for different measurement locations at the two different frequencies and two different buildings are compared and discussed for both line-of-sight and obstructed measurement locations. The uniform geometrical theory of diffraction was included in the model. However, for the measurement and prediction locations presented here, the diffracted rays do not contribute significantly to the received power delay profiles. It is likely that at other locations diffraction will significantly contribute to the received power delay profile. Hence, it is included in the model for completeness.

\subsubsection{Whittemore Hall $-1.3 \mathrm{GHz}$}

The floor plan of Whittemore Hall is shown in Figure 6.3-1. The transmitter was located at the intersection of two hallways, and is indicated by the letters ' $\mathrm{Tx}$ ' in the figure. The different receiver locations are indicated by the letters ' $A$ ' through ' $I$ '. These measurements were made with a direct pulse measurement system at $1.3 \mathrm{GHz}$ with a $4 \mathrm{~ns}$ rms pulse width resolution. Both the transmitter and receiver were stationary during measurements and each power delay profile represents an instantaneous 'snapshot' of the channel. Omni-directional vertically polarized discone antennas were used, and the measurements are described in more detail in [Haw91].

The measured and predicted power delay profiles for the line-of-sight receiver location $\mathrm{G}$ are shown in Figure 6.3-2. Notice that the path loss error is less than $2 \mathrm{~dB}$ and the rms delay spreads are quite similar. The amplitudes and arrival times of significant individual multipath components are predicted.

The measured and predicted power delay profiles for obstructed measurement location ' $\mathrm{H}$ ' are given in Figure 6.3-3. The path loss error is $0.3 \mathrm{~dB}$ and multipath components are predicted out to a total delay of $200 \mathrm{~ns}$. Although agreement is not as good as for line-of-sight locations, the large-scale change in path loss based on the change in topography has been tracked for this obstructed location. 


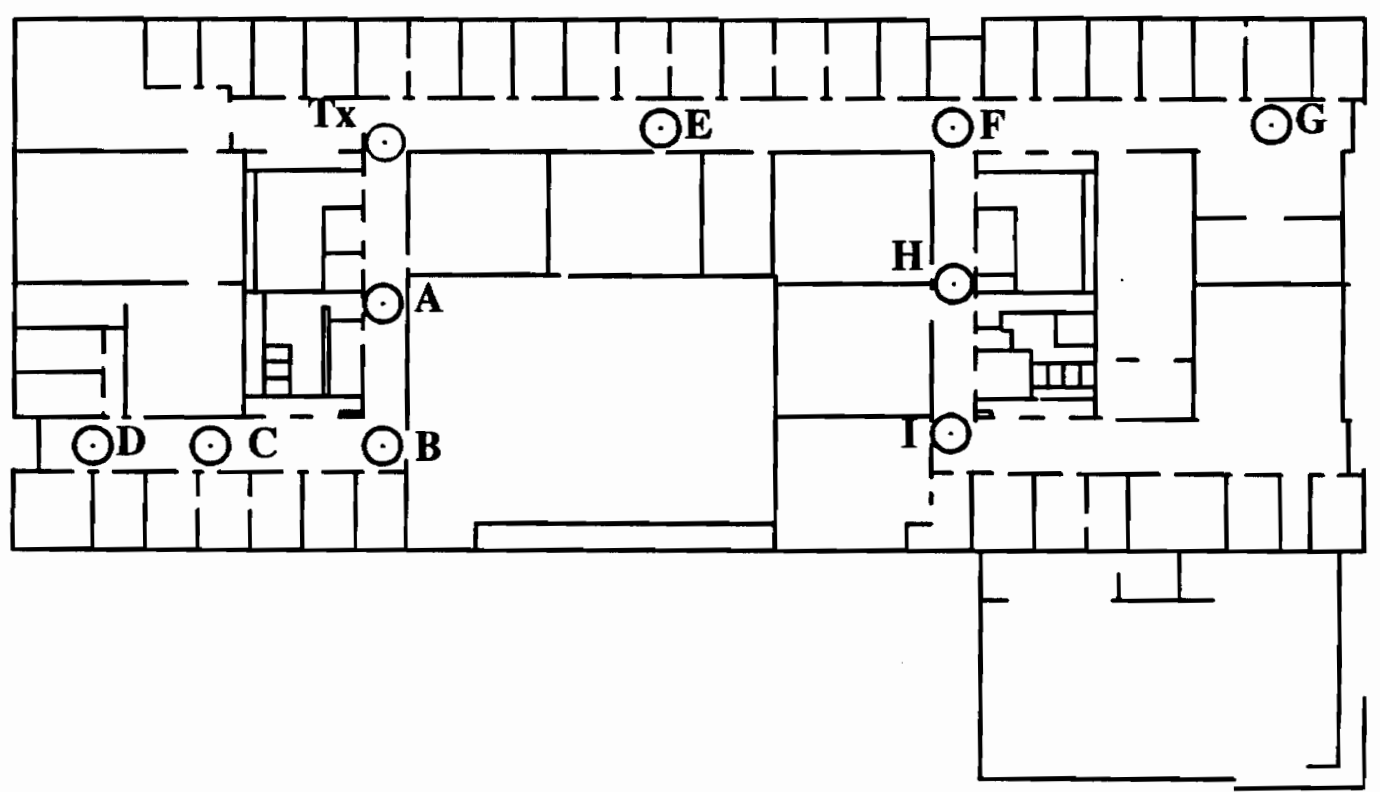

FIGURE 6.3-1. Measurement locations on the second floor of Whittemore Hall at 1.3 GHz. The transmitter is indicated by a ' $T x$ ', and the receiver locations are indicated by the letters ' $A$ ' through ' $I$ '.

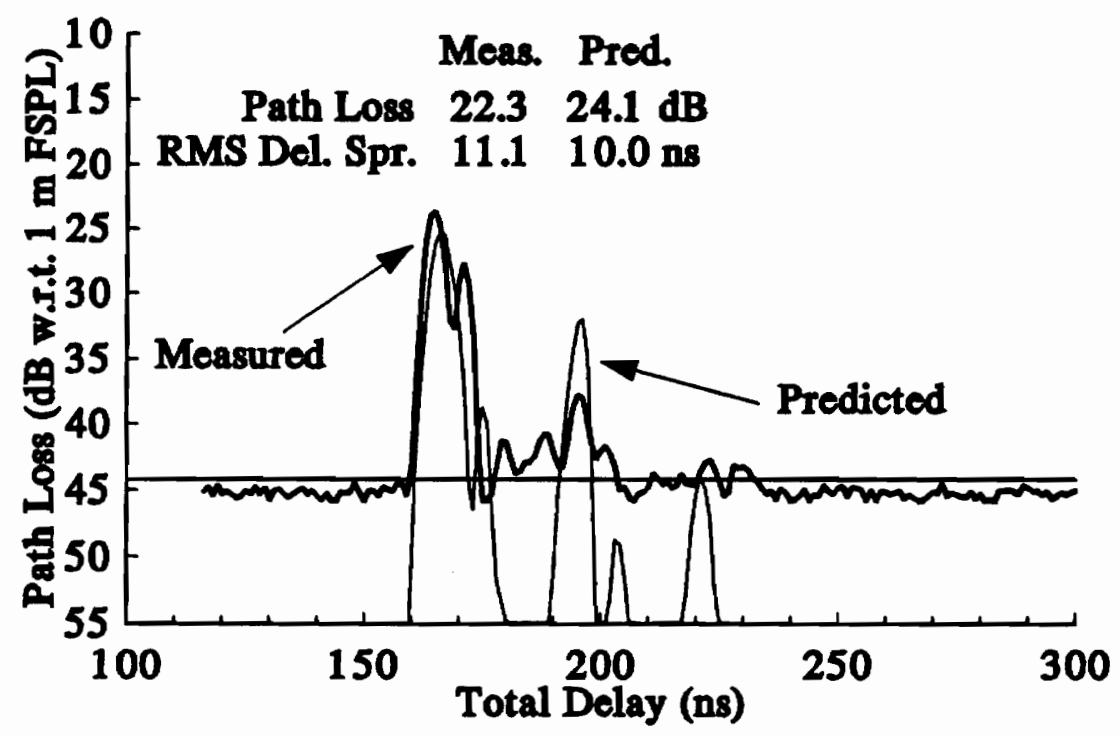

FIGURE 6.3-2. Measured and predicted power delay profiles at location $G$ in Whittemore Hall at $1.3 \mathrm{GHz}$. The path loss error is $1.8 \mathrm{~dB}$ and the rms delay spreads are nearly identical. 


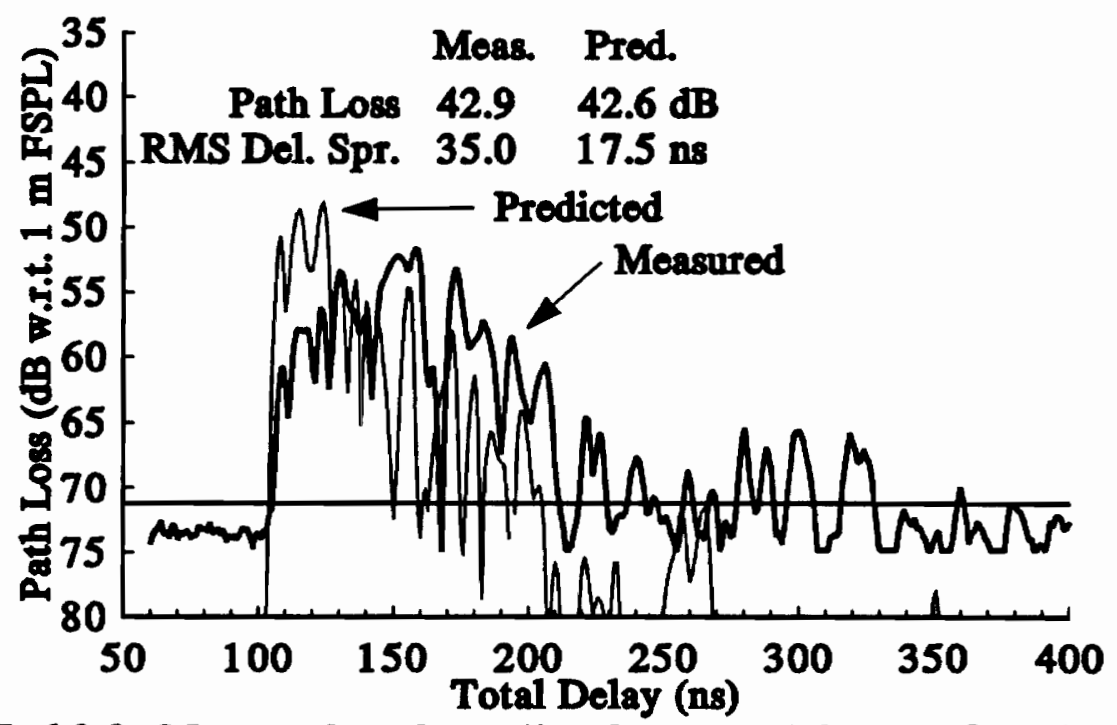

FIGURE 6.3-3. Measured and predicted power delay profiles for obstructed location ' $\mathrm{H}$ ' at $1.3 \mathrm{GHz}$ in Whittemore Hall. The path loss error is $0.3 \mathrm{~dB}$, and the arrival times of significant individual multipath components are predicted at total delays of up to $200 \mathrm{~ns}$.

Table 6.3-1 presents the measured and predicted path loss, rms delay spread, and delay interval for a window that contains $90 \%$ of the received energy for measurements made at $1.3 \mathrm{GHz}$ on the second floor of Whittemore Hall.

TABLE 6.3-1: Measured and predicted path loss, rms delay spread, and $90 \%$ delay interval at $1.3 \mathrm{GHz}$ on the second floor of Whittemore Hall.

\begin{tabular}{|c|c|c|c|c|c|c|}
\hline Hocation & Woast & rossorbs) & Measured & Predicted & 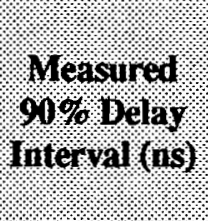 & $\begin{array}{l}\text { predotal } \\
\text { minteral }\end{array}$ \\
\hline WHL1AVV & 17.3 & 10.9 & 14.5 & 10.2 & 51 & 15 \\
\hline WHL1BVV & 19.4 & 13.3 & 7.9 & 7.5 & 17 & 25 \\
\hline WHO1CVV & 42.0 & 51.6 & 23.4 & 11.5 & 68 & 38 \\
\hline WHO1DVV & 50.2 & 45.5 & 14.1 & 9.5 & 41 & 23 \\
\hline WHL1EVV & 17.8 & 16.6 & 35.6 & 5.1 & 27 & 15 \\
\hline WHL1FVV & 23.9 & 19.3 & 43.4 & 22.9 & 77 & 15 \\
\hline WHL1GVV & 22.3 & 24.1 & 11.1 & 10.0 & 32 & 33 \\
\hline WHO1HVV & 42.9 & 42.6 & 35.0 & 17.5 & 91 & 59 \\
\hline WHO1IVV & 47.1 & 52.7 & 33.7 & 25.1 & 91 & 70 \\
\hline
\end{tabular}




\subsubsection{Whittemore Hall - 4.0 GHz}

Additional measurements were made on the second floor of Whittemore Hall at 4.0 $\mathrm{GHz}$ with the spread spectrum channel sounder described in Section 2.5 Multipath Propagation Measurements. The channel sounder chip rate was $240 \mathrm{MHz}$ which yielded a time resolution of about $8 \mathrm{~ns}$. Omni-directional bi-conical antennas were used at the transmitter and receiver. The transmitter location was the same as for the $1.3 \mathrm{GHz}$ measurements. The receiver was moved to different locations in the hallway throughout the same measurement area. The transmitter and receiver measurement locations are indicated in Figure 6.34. For measurements at $4.0 \mathrm{GHz}$, the transmitter remained stationary, and the receiver recorded five instantaneous power delay profiles over a path length of about one meter. Each instantaneous power delay profile was recorded while the receiver was stationary, and the five power delay profiles were averaged to provide a spatial average that eliminates multipath fading within one pulse period.

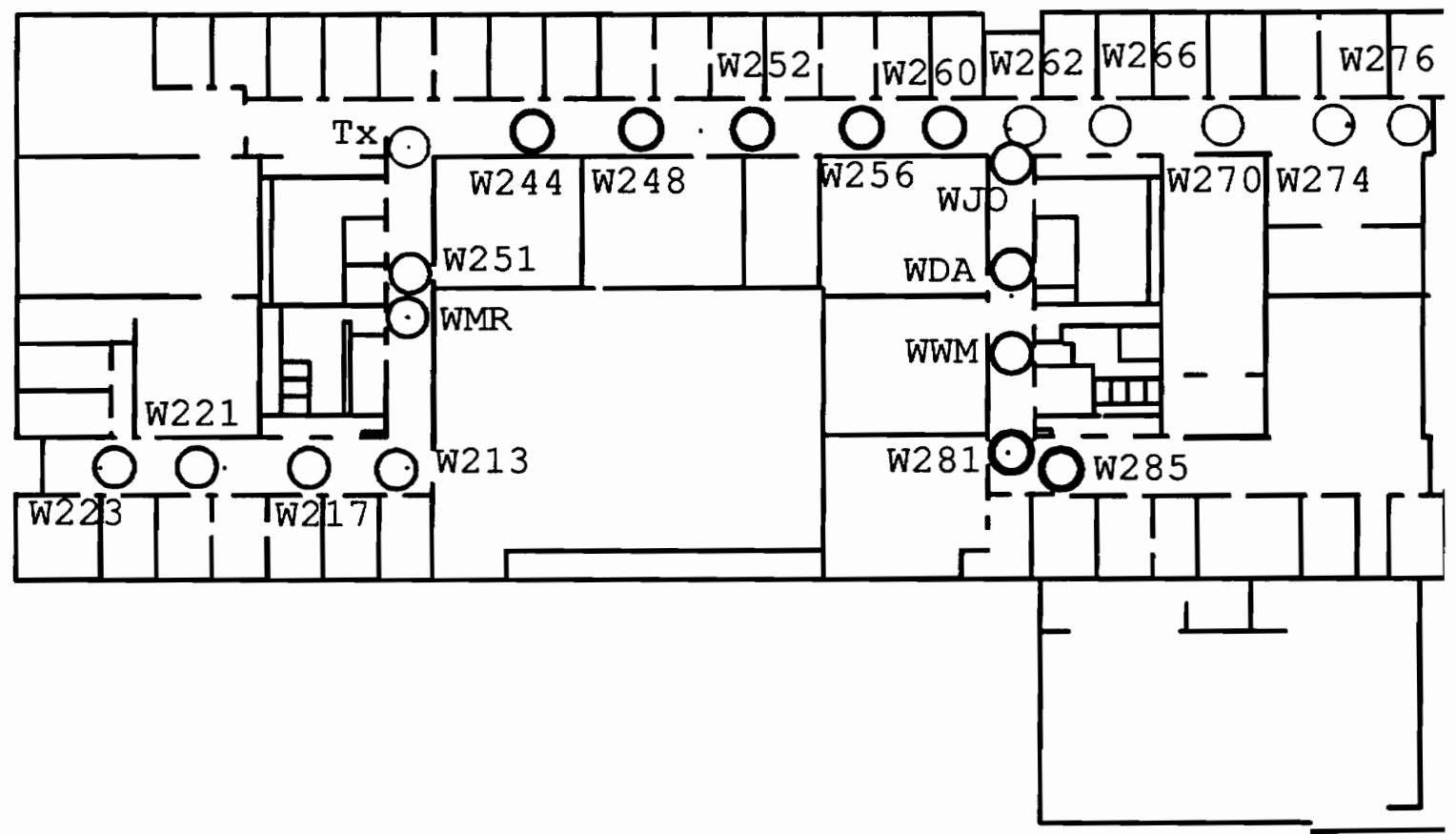

FIGURE 6.3-4. Building blueprint of the second floor of Whittemore Hall that shows where power delay profile measurements were made at $4.0 \mathrm{GHz}$ with a spread spectrum channel sounder with a $8 \mathrm{~ns}$ pulse resolution. 
Consider the line-of-sight receiver measurement location W251 shown in Figure 6.3-5. The path loss error is less than one decibel and the rms delay spreads are quite similar. There is good agreement between the measured and predicted power delay profiles as the prediction includes the amplitudes and arrival times of many significant multipath components. A table of measured and predicted path loss, rms delay spread, and $90 \%$ delay interval is given in Table 6.3-2. Notice that the measured and predicted path loss at

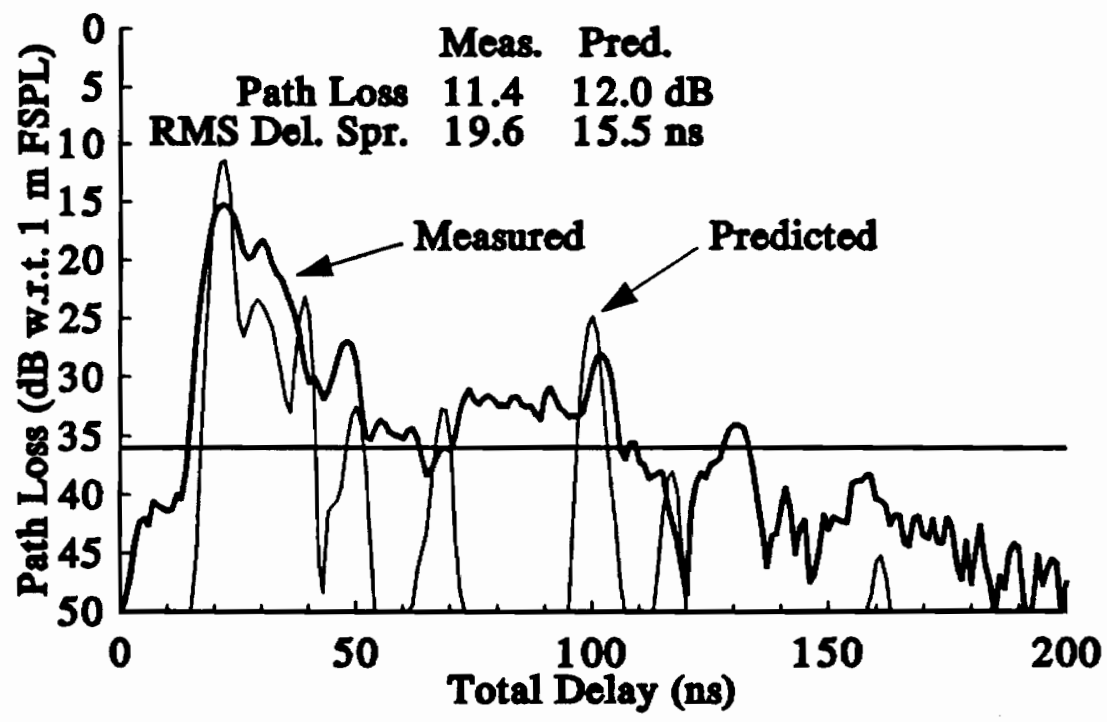

FIGURE 6.3-5. Measured and predicted power delay profiles at receiver measurement location W251 in Whittemore Hall at 4.0 GHz. The path loss error is less than 1.0 dB and the rms delay spreads are quite similar.

location W262 is on the order of $15 \mathrm{~dB}$ greater than the free space path loss at one meter for this line-of-sight location. Consider the obstructed locations WJO and WDA. The comparison of measured and predicted power delay profiles is given in Figure 6.3-6 and Figure 6.3-7, respectively. The significance of these locations is that as the receiver is moved from a line-of-sight position to an obstructed position, the predicted power delay profiles accurately tracks the $16 \mathrm{~dB}$ change in path loss. The change in path loss from 31 $\mathrm{dB}$ to $38 \mathrm{~dB}$ relative to one meter as the receiver moves from WJO to WDA is also predicted. Hence, major changes in propagation characteristics are correctly tracked as the receiver undergoes significant changes in the surroundings. 
TABLE 6.3-2: Measured and Predicted path loss, rms delay spread, and $90 \%$ delay interval at $4.0 \mathrm{GHz}$ on the second floor of Whittemore Hall.

\begin{tabular}{|c|c|c|c|c|c|c|}
\hline 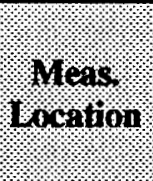 & $\begin{array}{l}\text { Meas } \\
\text { rath } \\
\text { (as) }\end{array}$ & $\begin{array}{l}\text { Pred: } \\
\text { Patlis } \\
\text { grosis) }\end{array}$ & $\begin{array}{l}\text { Mersured } \\
\text { Rins Delar } \\
\text { Spread (ns) }\end{array}$ & 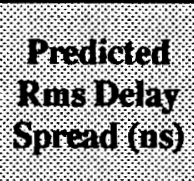 & moveroved & 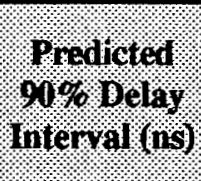 \\
\hline W223 & 38.8 & 45.5 & 25.9 & 9.9 & 70 & 33 \\
\hline W221 & 37.9 & 45.4 & 22.9 & 10.9 & 69 & 34 \\
\hline W217 & 33.4 & 37.1 & 18.4 & 10.2 & 53 & 32 \\
\hline W213 & 14.3 & 16.9 & 11.5 & 13.2 & 37 & 48 \\
\hline WMR & 12.5 & 10.5 & 20.0 & 11.3 & 65 & 19 \\
\hline W251 & 11.4 & 12.0 & 19.6 & 15.5 & 68 & 21 \\
\hline W244 & 10.6 & 11.1 & 10.9 & 3.0 & 32 & 10 \\
\hline W248 & 13.4 & 14.7 & 17.7 & 8.6 & 38 & 14 \\
\hline W252 & 11.3 & 21.4 & 4.0 & 33.1 & 9 & 15 \\
\hline W256 & 19.2 & 20.3 & 36.0 & 44.1 & 51 & 199 \\
\hline W260 & 19.2 & 14.4 & 25.9 & 33.6 & 31 & 11 \\
\hline W262 & 17.5 & 12.5 & 16.3 & 34.8 & 14 & 143 \\
\hline W266 & 19.8 & 12.0 & 19.1 & 12.5 & 40 & 8 \\
\hline W270 & 20.7 & 14.6 & 19.5 & 18.3 & 73 & 74 \\
\hline W274 & 22.7 & 24.6 & 15.8 & 15.3 & 47 & 39 \\
\hline W276 & 27.6 & 25.6 & 42.6 & 30.5 & 52 & 137 \\
\hline WJO & 29.9 & 31.6 & 31.9 & 40.6 & 76 & 147 \\
\hline WDA & 38.3 & 38.3 & 45.2 & 12.4 & 154 & 29 \\
\hline WWM & 43.8 & 46.3 & 50.4 & 12.9 & 162 & 41 \\
\hline W281 & 47.6 & 50.2 & 38.5 & 31.0 & 140 & 114 \\
\hline W285 & 56.0 & 44.4 & 26.5 & 7.8 & 80 & 5 \\
\hline
\end{tabular}

\subsubsection{Norris Hall - 4.0 GHz}

The locations of the transmitters and receivers for $4.0 \mathrm{GHz}$ measurements in Norris Hall are shown in Figure 6.3-8. Some measurements were made with the transmitter at location ' $\mathrm{J}$ ' where the two hallways intersect, and others were made with the transmitter at location ' $B$ ' in one of the hallways. The receiver was located at the locations indicated by the letters ' $A$ ' through ' $J$ '. The measurement equipment and procedure were identical to the $4.0 \mathrm{GHz}$ measurements made in Whittemore Hall. 


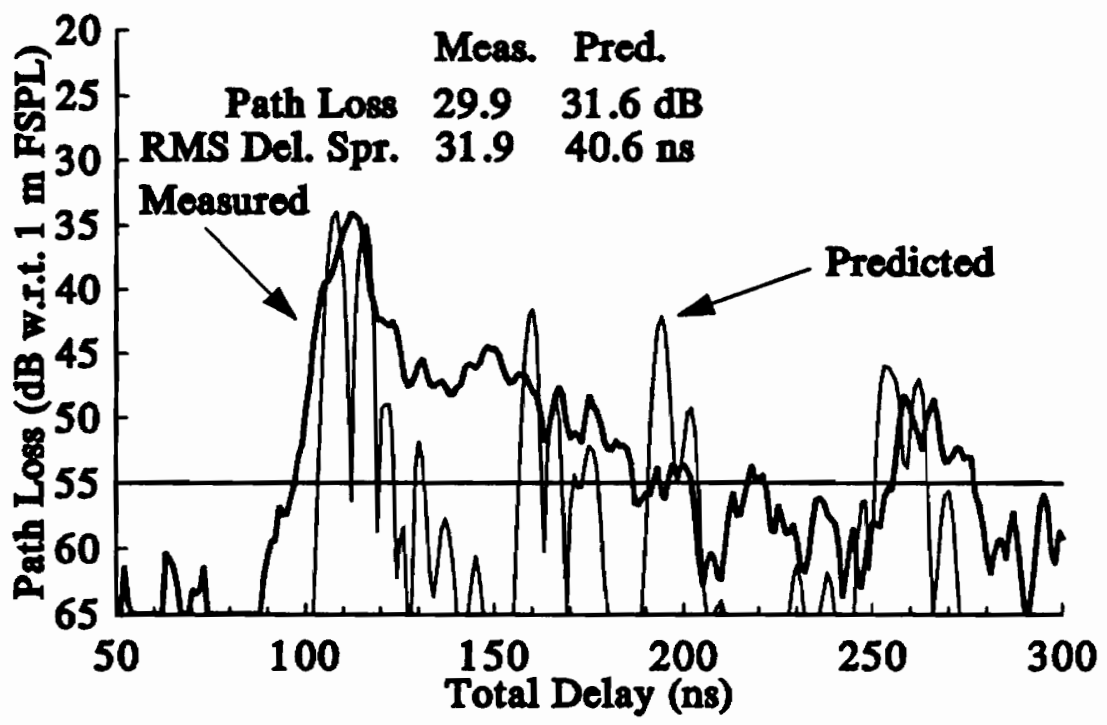

FIGURE 6.3-6. Comparison of measured and predicted power delay profiles in Whittemore Hall at $4.0 \mathrm{GHz}$ at receiver location WJO.

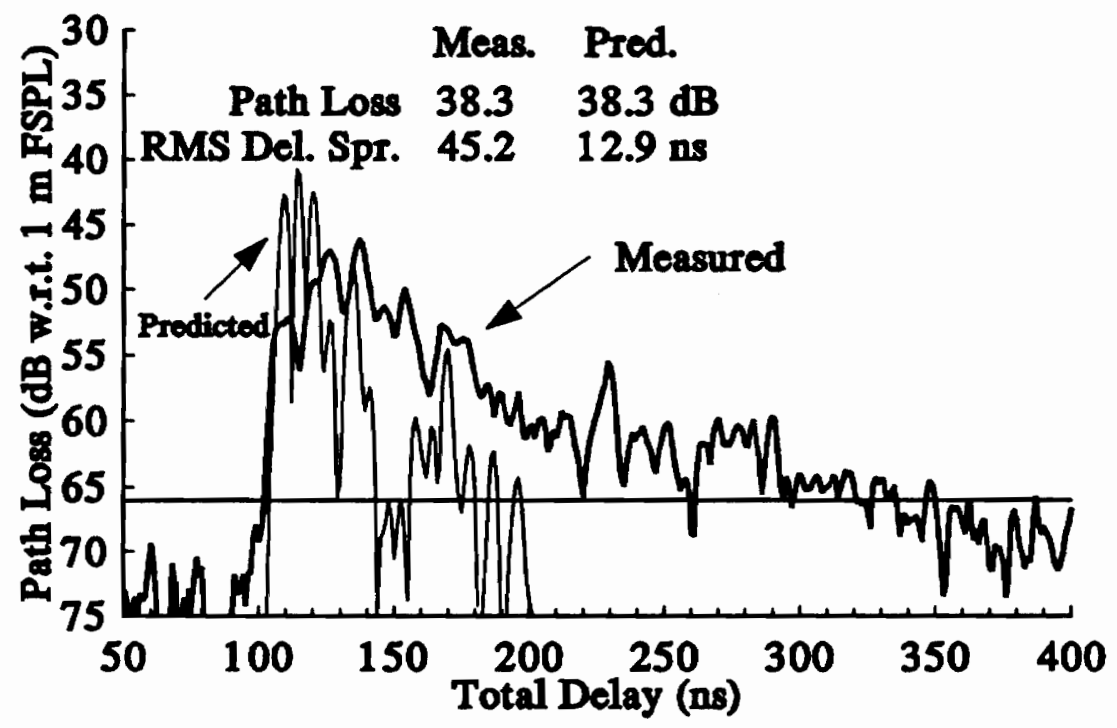

FIGURE 6.3-7. Measured and predicted power delay profiles for the obstructed location WDA in Whittemore Hall at 4.0 GHz.

Consider the transmitter at location ' $\mathrm{J}$ ' and the receiver at locations ' $\mathrm{F}$ ' and ' $\mathrm{H}$ '. The measured and predicted power delay profiles for location ' $F$ ' are shown in Figure 6.39, and the profiles for location ' $\mathrm{H}$ ' are shown in Figure 6.3-10. For both line-of-sight locations, the amplitudes and arrival times of significant multipath components are predicted and the path loss errors are less than $4.1 \mathrm{~dB}$. At location ' $\mathrm{F}$ ', the rms delay spreads are 


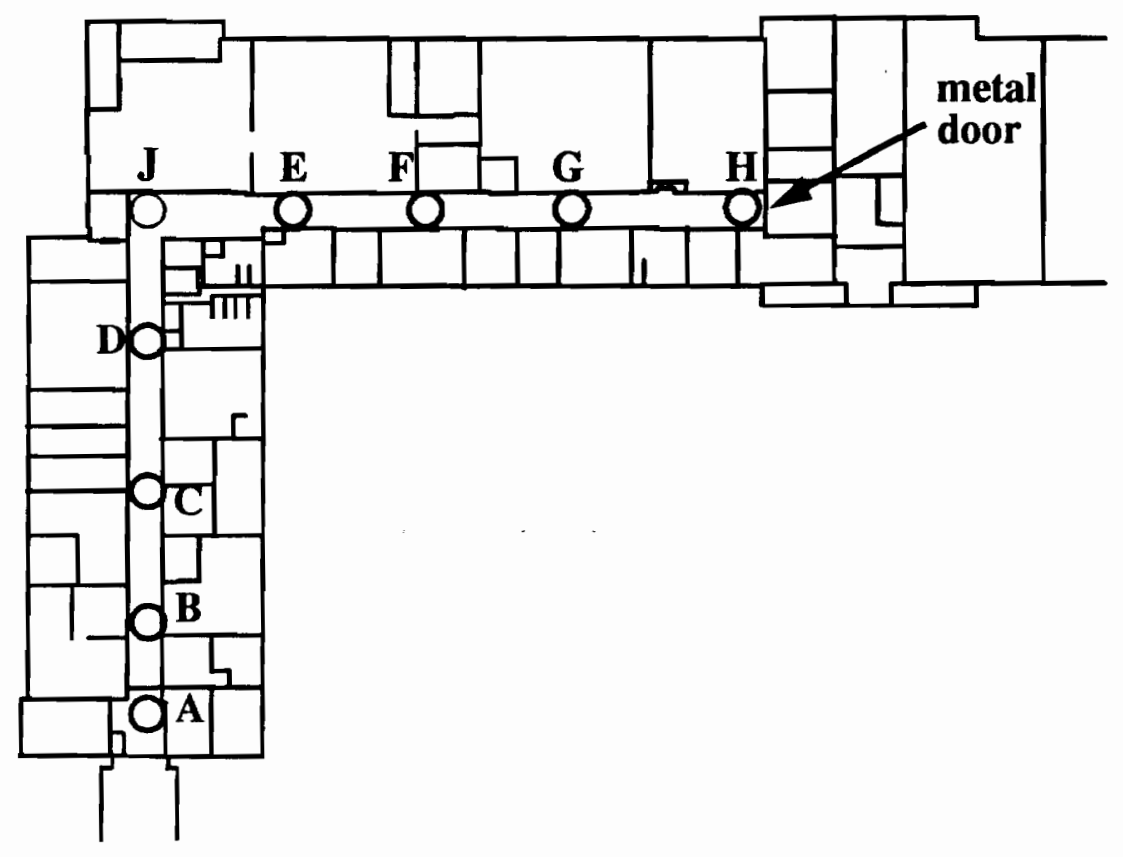

FIGURE 6.3-8. Building blueprint of Norris Hall that shows the transmitter and receiver locations where power delay profiles were recorded at $4.0 \mathrm{GHz}$. At the end of the hallway near measurement location ' $H$ ' was a large metal door. Measurements were made with the transmitter at locations ' $J$ ' and ' $B$ '.

quite similar since the amplitudes of measured and predicted multipath components in the power delay profiles are nearly identical at almost all excess delays. At location ' $\mathrm{H}$ ', the rms delay spread varies considerably, but this parameter is sensitive to small changes in the power delay profile such as the $8 \mathrm{~dB}$ difference in the first arriving multipath component. However, the predicted power delay profile accurately predicts the arrival times of the multipath components and predicts a path loss within $4.1 \mathrm{~dB}$ of the measured value.

Consider now the measurement location with the transmitter moved from location ' $J$ ' to location ' $B$ ' with the receiver remaining at location ' $H$ ' at the end of the hallway next to the metal door. The measurement topography changes from line-of-sight to heavily obstructed. In fact, the direct path passes outside the building and re-enters in the other wing of the building. The measured and predicted power delay profiles are shown in Figure 6.3-11. Notice that the path loss error is less than one decibel and many of the amplitudes and arrival times of significant multipath components are predicted. The significance of this prediction is that as the transmitter was moved from a line-of-sight location to an 


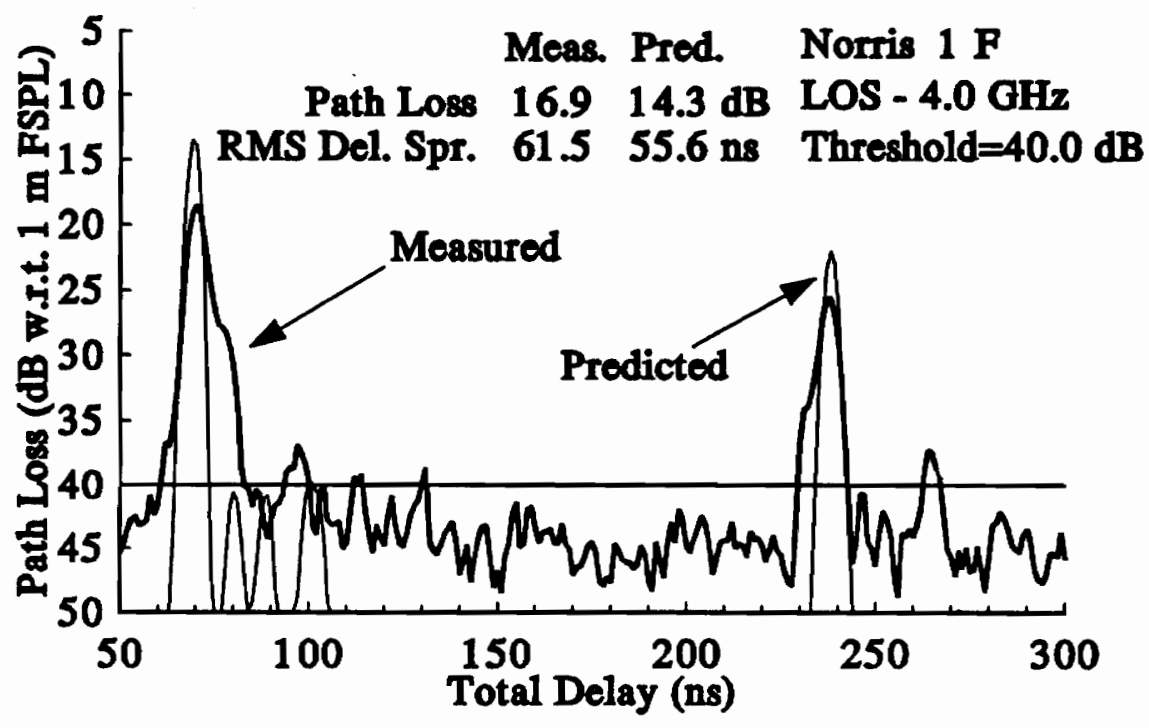

FIGURE 6.3-9. Measured and predicted power delay profiles for transmitter location ' $\mathrm{J}$ ' and receiver Iocation ' $\mathrm{F}$ ' in Norris Hall at 4.0 GHz.

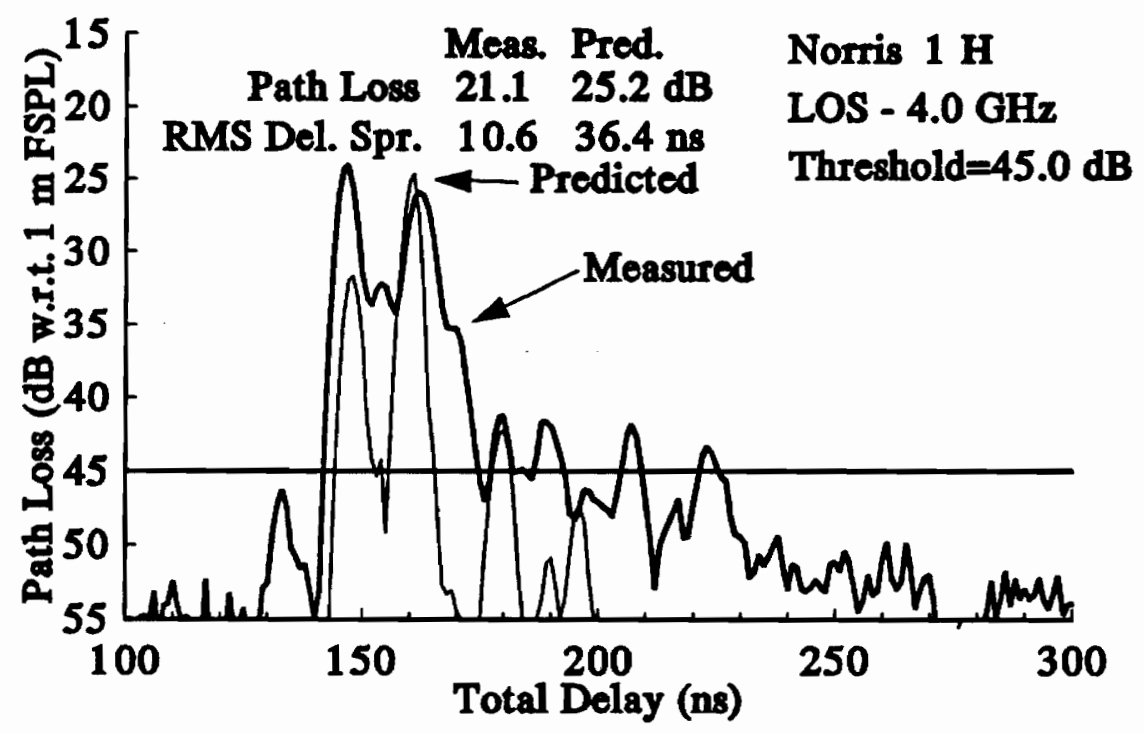

FIGURE 6.3-10. Measured and predicted power delay profiles for transmitter location ' $J$ ' and receiver location ' $H$ ' in Norris Hall at 4.0 GHz.

obstructed location, the predicted path loss remained within several decibels of the measured path loss over a path loss change of $30 \mathrm{~dB}$. Thus, changes in the propagation characteristics as a function of major changes in the surroundings of the transmitter and receiver have been accurately modeled with this ray tracing propagation method. A table of values for measured and predicted path loss, rms delay spread, and $90 \%$ delay interval is given in 
Table 6.3-3. The second digit in the name of the measurement location with the transmitter at location ' $\mathrm{J}$ ' is 1 , and the second digit in the name of the measurement location with the transmitter at location ' $\mathrm{B}$ ' is 2 . The third digit is the receiver location and the first digit represents that the measurement were made in Norris Hall.

TABLE 6.3-3: Measured and Predicted path loss, rms delay spread, and 90\% delay interval at 4.0 GHz on the first floor of Norris Hall.

\begin{tabular}{|c|c|c|c|c|c|c|}
\hline mogation & $\begin{array}{l}\text { Meas } \\
\text { patti } \\
\text { yoss } \\
(\mathrm{dib})\end{array}$ & $\begin{array}{l}\text { Pred } \\
\text { Path } \\
\text { ross } \\
(\mathrm{dBr})\end{array}$ & $\begin{array}{l}\text { Measured } \\
\text { Rims Delay } \\
\text { Sprear (n) }\end{array}$ & $\begin{array}{l}\text { Predicted } \\
\text { Rrms Delay } \\
\text { Spread (ns) }\end{array}$ & 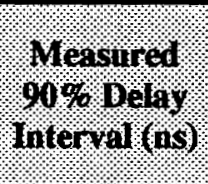 & $\begin{array}{l}\text { predicted } \\
\text { oro\% Delay } \\
\text { interval (ius) }\end{array}$ \\
\hline M1A & 25.6 & 23.3 & 10.2 & 19.1 & 27 & 15 \\
\hline M1B & 19.3 & 22.4 & 5.1 & 7.4 & 14 & 116 \\
\hline M1C & 19.0 & 12.0 & 12.0 & 5.4 & 27 & 11 \\
\hline M1D & 18.2 & 8.5 & 30.9 & 7.4 & 51 & 9 \\
\hline M1E & 13.8 & 14.7 & 16.4 & 37.1 & 19 & 18 \\
\hline M1F & 16.9 & 14.3 & 61.5 & 55.6 & 171 & 171 \\
\hline M1G & 21.1 & 18.5 & 46.1 & 34.4 & 105 & 100 \\
\hline M1H & 21.1 & 25.2 & 10.6 & 36.4 & 24 & 16 \\
\hline M2C & 19.9 & 15.6 & 18.6 & 14.6 & 52 & 37 \\
\hline M2D & 18.9 & 21.0 & 16.7 & 34.6 & 69 & 78 \\
\hline M2E & 55.0 & 58.3 & 34.4 & 41.0 & 72 & 148 \\
\hline M2F & 59.8 & 58.3 & 60.9 & 36.6 & 171 & 109 \\
\hline M2G & 59.1 & 61.8 & 22.4 & 20.5 & 69 & 60 \\
\hline $\mathrm{M} 2 \mathrm{H}$ & 59.2 & 58.8 & 17.7 & 32.2 & 53 & 80 \\
\hline M2J & 22.4 & 21.2 & 12.9 & 6.5 & 38 & 13 \\
\hline
\end{tabular}

\subsection{Comparison of Measured and Predicted Channel Parameters}

Although path loss, rms delay spread, and delay interval are not necessarily appropriate as optimization criteria, they are important channel parameters that are commonly used to characterize the propagation channel. Hence, a quantization of the accuracy of the model in these areas is required. The parameters are computed from the measured and predicted power delay profiles where the predicted power delay profiles were computed from the effective building materials optimized over the ensemble of locations in a given building at a given frequency. For each combination, a loss tangent of 0.0015 was used and the 


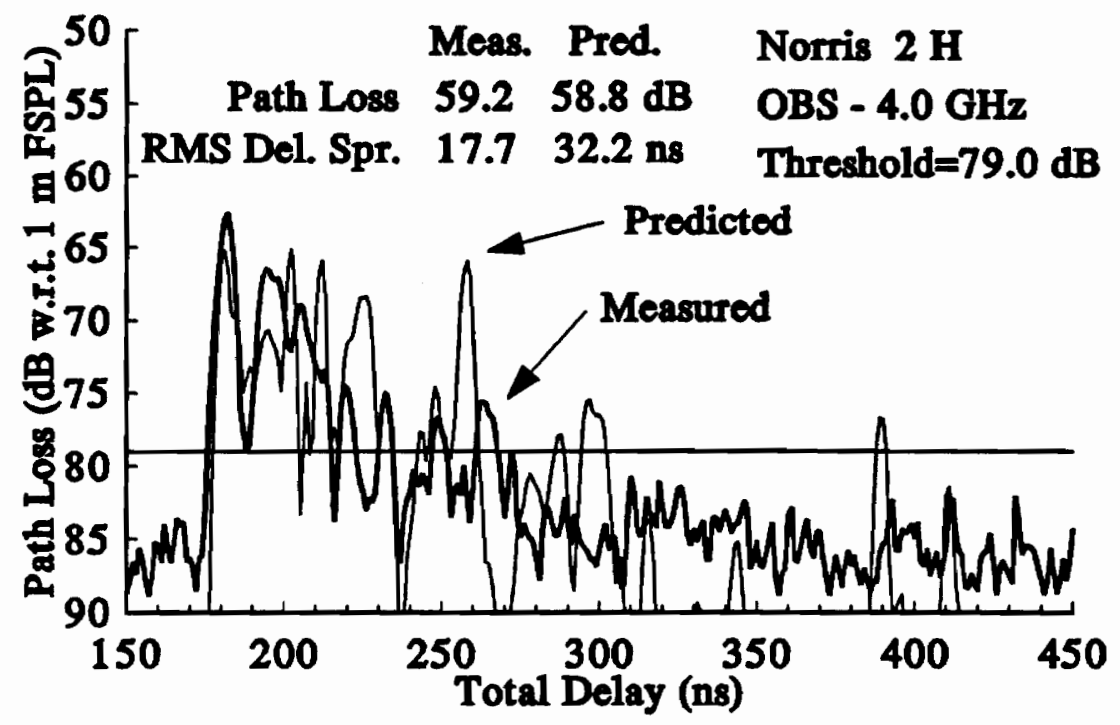

FIGURE 6.3-11. Measured and predicted power delay profiles in Norris Hall at 4.0 GHz. The transmitter was at location ' $B$ ', and the receiver was at location ' $H$ '. relative permittivity was 4.4 for both sets of predictions in Whittemore Hall and was 7.4 for predictions in Norris Hall. Figure 6.4-1 shows a scatter plot of measured and predicted path loss for all measurement locations. The standard deviation of path loss error is $4.8 \mathrm{~dB}$ for all measurement locations. This standard deviation is obtained over a dynamic range of path loss that ranges from $10 \mathrm{~dB}$ to $60 \mathrm{~dB}$ above the path loss along a one meter free space path. The individual path loss scatter points are clustered about the diagonal line that indicates where measured and predicted path loss are identical. This result shows the validity of the ray tracing propagation prediction model for path loss coverage prediction in a building. The model accurately tracks large changes in path loss as a function of the surroundings of the transmitter and receiver.

A scatter plot of the rms delay spread for the measured and predicted locations is shown in Figure 6.4-2. The rms delay spread calculations show large errors at some locations. However, rms delay spread can be extremely sensitive to small changes in the power delay profile. Only samples above the threshold were used to compute the statistics. If the predicted component is below the threshold, it is not counted, even though the difference between the measured and predicted power delay profiles is small. For example, examine the measured and predicted profiles for location WHL1EVV in Whittemore Hall shown in 


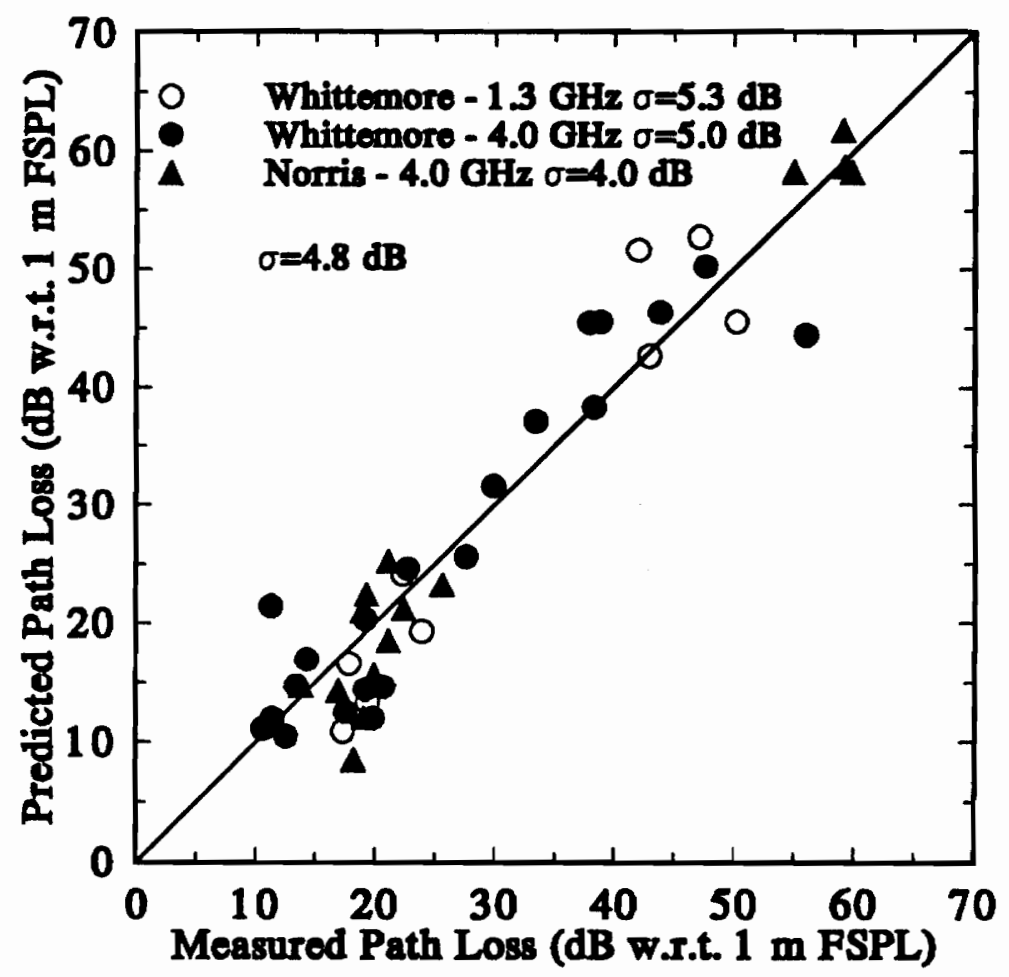

FIGURE 6.4-1. Scatter plot of measured and predicted path loss for the three sets of measurements in Whittemore Hall at 1.3 and 4.0 GHz and in Norris Hall at 4.0 GHz. The standard deviation of path loss prediction error is $4.8 \mathrm{~dB}$ over all measurement locations.

Figure 6.4-3. The rms delay spread error is large, but in Figure 6.4-3, it can be clearly seen that several multipath components are predicted with amplitudes below the threshold that must be used because of the dynamic range of the measurements. Hence, the difference between measured and predicted power delay profiles is not as large as the difference in rms delay spread indicates. Similar results can be seen at location WHL1FVV in Whittemore, and location M1E in Norris Hall.

Consider the COST 207 delay interval statistics. The delay interval is a measure of the span of excess delay that contains a certain portion of the received energy. This is a parameter that considers the arrival time and amplitude of the multipath energy that is less sensitive to small changes in power delay profiles than is rms delay spread. Delay intervals can be computed for any amount of received energy. Here, the measured and predicted delay intervals for $90 \%, 75 \%$, and $50 \%$ of the received energy are computed. Figure 6.4-4 gives the scatter plot of delay interval for $90 \%$ of the received energy. Most of the delay 


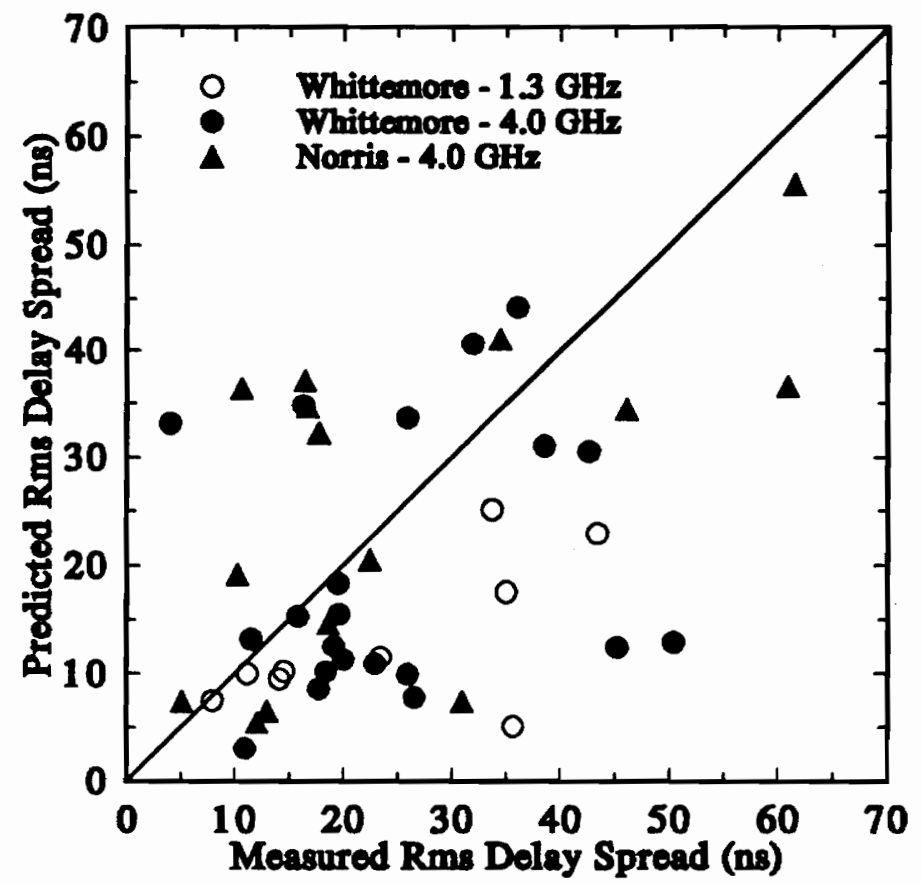

FIGURE 6.4-2. Scatter plot of measured and predicted rms delay spread for all three measurement combinations.

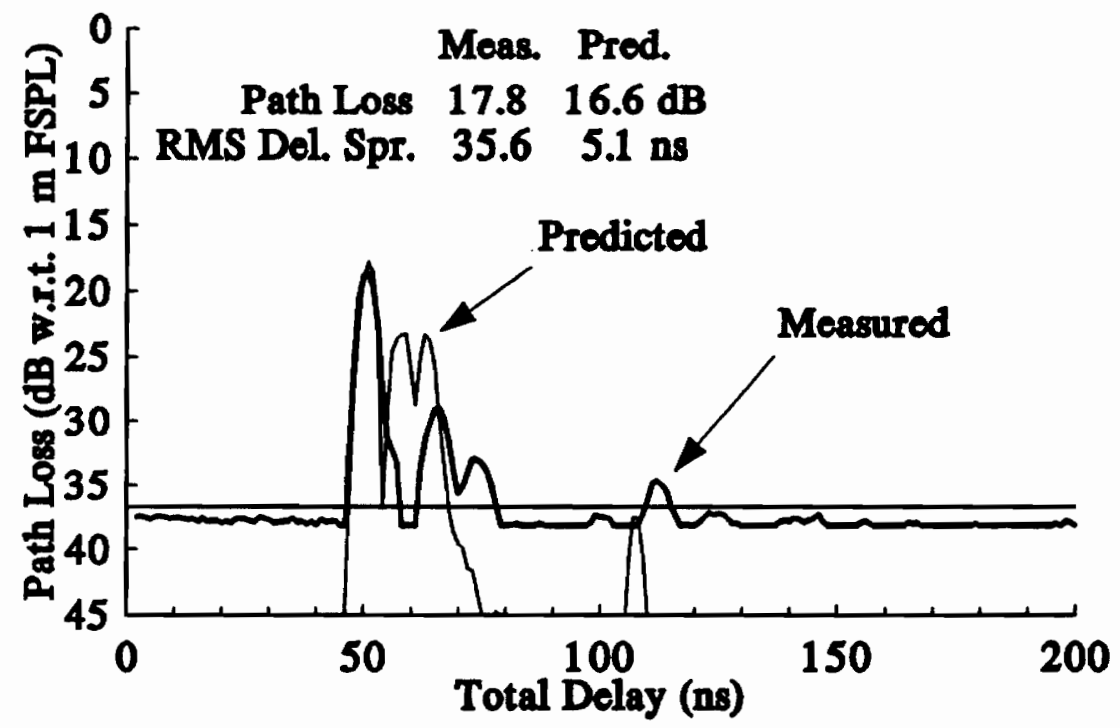

FIGURE 6.4-3. Measured and predicted power delay profiles for location WHL1EVV in Whittemore Hall. Notice that the predicted component at 110 ns total delay is below the threshold leading to a large discrepancy between the measured and predicted rms delay spread. 
intervals are less than $100 \mathrm{~ns}$ and are clustered about the diagonal line that indicates where measured and predicted delay interval window sizes are equal. There are several locations where the measured and predicted delay intervals differ significantly, but in general, the 90\% delay interval scatter plot is more clustered than the rms delay spread scatter plot shown in Figure 6.4-2. The scatter plot of 75\% of the received energy is given in Figure 6.4-5, and the scatter plot for 50\% of the received energy is given in Figure 6.4-6. The span of values for the $75 \%$ and $50 \%$ delay intervals decreases and becomes more clustered about the diagonal (measured=predicted) line as the amount of energy in the window decreases.

Window Size for $90 \%$ of Received Power

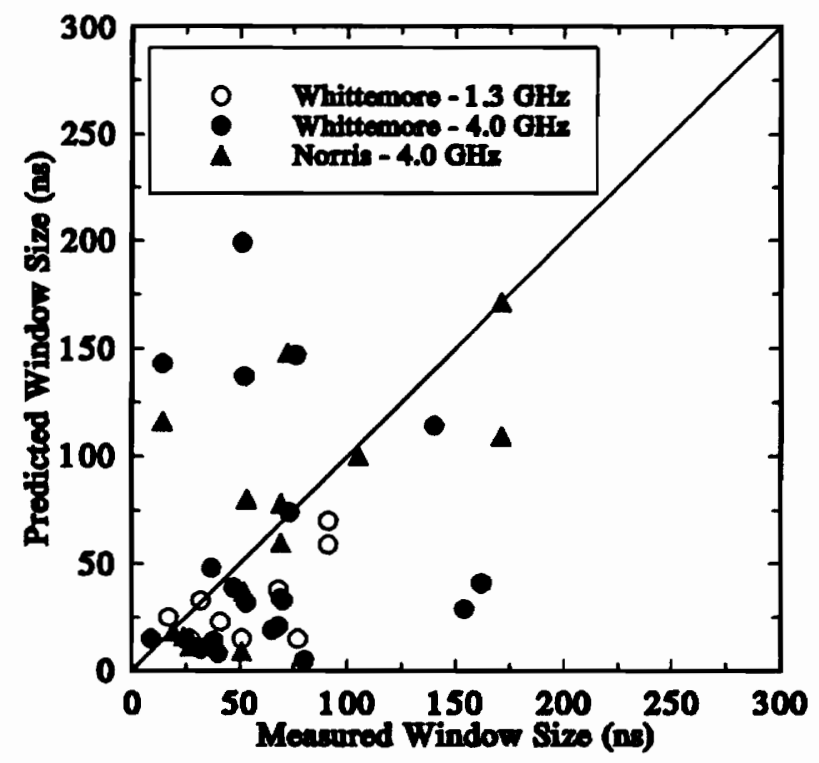

FIGURE 6.4-4. Scatter plot of the measured and predicted COST 207 delay interval for $90 \%$ of the received energy.

\subsection{Summary}

This chapter has detailed the results of the ray tracing site-specific propagation model. Previous work in propagation prediction has not focused on comparison of measured and predicted propagation data that includes the multipath arrival time and a specific location-by-location comparison of the measured and predicted data. Hence, a method for 
Window Size for $\mathbf{7 5} \%$ of Received Power

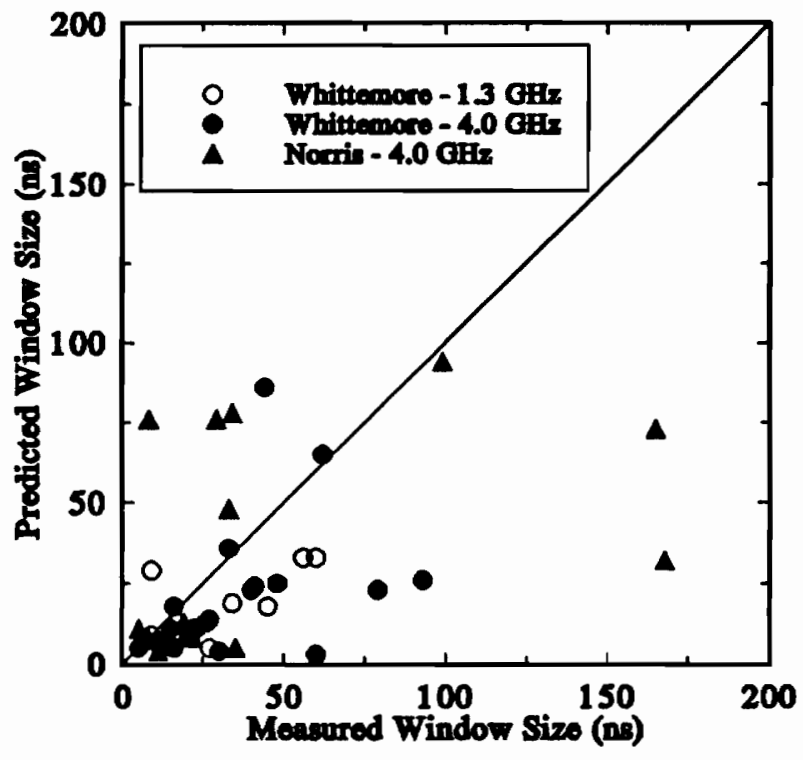

FIGURE 6.4-5. Scatter plot of the measured and predicted COST 207 delay interval for $75 \%$ of the received energy.

Window Size for $50 \%$ of Received Power

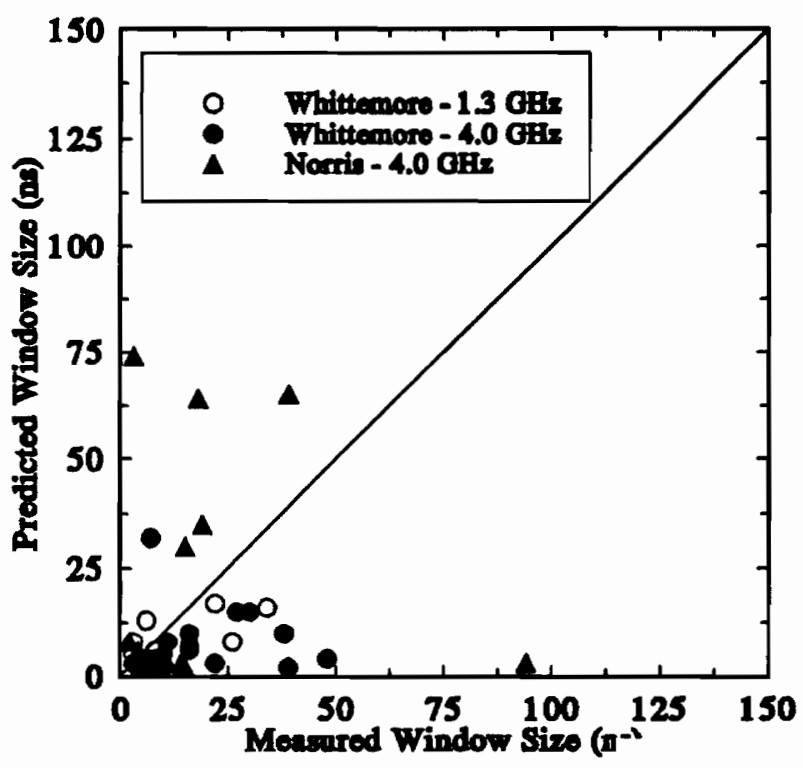

FIGURE 6.4-6. Scatter plot of the measured and predicted COST 207 delay interval for $50 \%$ of the received energy. 
quantifying the difference between measured and predicted power delay profiles has been developed. The area under the square error function as a function of excess delay has been minimized to provide predicted power delay profiles and determine effective building material properties. The effective building material properties were determined for each combination of building and frequency. Optimization revealed that the effective relative dielectric constants are 4.4 at both 1.3 and $4.0 \mathrm{GHz}$ in Whittemore Hall, and 7.4 at 4.0 $\mathrm{GHz}$ in Norris Hall. These values are similar to the values of other materials that could possibly used in building construction. However, the use of an effective relative dielectric constant is only a first-order approximation since all objects were considered homogeneous and identical. Ideally, the exact material properties for each object would be known.

Qualitative comparison of power delay profiles showed that the amplitudes and arrival times of individual multipath components were predicted in both line-of-sight and obstructed topographies. In both buildings, as a receiver was moved from a line-of-sight location to an obstructed location, the predicted power delay profiles accurately tracked the change in path loss. This change was $16 \mathrm{~dB}$ in one building and $30 \mathrm{~dB}$ in the other. Overall, the standard deviation of the path loss error is $4.8 \mathrm{~dB}$ over a dynamic range of 50 $\mathrm{dB}$. Comparison of the rms delay spread and COST 207 delay interval indicates that in many cases the time delay parameters of measured and predicted power delay profiles are similar. 


\section{Conclusions and Future Work}

\subsection{Conclusions}

This work has presented a site-specific ray tracing method for predicting radio wave propagation in buildings. Chapter 1 discussed the recent growth of wireless communications and why accurate propagation prediction models are needed. Chapter 2 described multipath propagation and its effects on transmitted signals. Several methods of measuring radiowave propagation in multipath radio channels were detailed. Both wide band and narrow band measurement techniques and data processing methods were discussed. Multipath channel parameters such as path loss, rms delay spread, and delay interval were defined.

Chapter 3 discussed propagation models based on measurements in many different building environments. Results for path loss and delay spread were presented as a function of building environment and frequency. The models discussed in Chapter 3 progressed from statistical models to site-specific models that incorporate elements of the building surroundings into the model. In particular, models for path loss caused by floors between transmitters and receivers, partitions, and other common obstructing objects were presented.

The fundamental theory for site-specific propagation modeling was outlined in Chapter 4. The theory of geometrical optics was presented for application in an automated ray tracing program. Scattering and diffraction from fiat plates were computed using the uniform geometrical theory of diffraction, physical optics, and a heuristic incoherent scattering model based on radar cross-section. The applicability of each model to site-specific propagation prediction was discussed. The uniform geometrical theory of diffraction was shown to be the most accurate for a wide range of scattering angles.

Chapter 5 described the ray tracing technique based on geometrical optics in detail for prediction of radiowave propagation in buildings. Line-of-sight paths, specularly reflected and transmitted paths, and diffracted paths were included in the model. A build- 
ing database manager and data processing of the raw ray tracing program output were detailed.

The comparison of measured and predicted propagation in Chapter 6 verified the use of this ray tracing propagation model for prediction power delay profiles for a transmitter and receiver located on a single floor within a building. Propagation parameters such as path loss, rms delay spread, and delay interval can be computed from the power delay profiles. The standard deviation of path loss error is $4.8 \mathrm{~dB}$ and qualitative comparison of measured and predicted power delay profiles show good time delay agreement. The results presented here indicate that this model can be used to predict power delay profiles in many different buildings based on site-specific information such as wall location and building materials. Suggestions for future efforts in ray tracing are given below.

\subsection{Future Work}

This work has presented a geometrical optics model for predicting multipath power delay profiles inside buildings using a site-specific building database. This dissertation has shown that this model is successful for predicting the propagation inside two buildings on the Virginia Tech campus. One extension of this work would be to test and refine this ray tracing method by measuring and predicting propagation in a large number of additional buildings. This model has been validated for single-floor measurements only. A suggested extension is to extend the region of validity of the ray tracing program to predict the case of propagation between a transmitter and receiver located on different floors within a building. Even for measurements on the same floor in the same building, there is still some discrepancy between the measured and predicted power delay profiles. A detailed investigation into the error mechanisms could prove useful for improving the prediction accuracy.

This work has been the first to compare the time information in measured and predicted power delay profiles for specific locations. Path loss, rms delay spread, and the delay interval have been used as parameters to quantify statistical differences between measurements and predictions. The optimization of effective building material properties 
has been performed based on a point-by-point error function as a function of excess delay. This optimization has attempted to reduce the 'distance' in some sense between the two curves. The development of a statistic, parameter, or function that quantifies the error between measured and predicted power delay profiles that provides insight into the source of the error would be extremely useful in the development of more accurate propagation models. This method should be able to identify arrival time as well as amplitude difference between the measured and predicted power delay profile.

There are a few specific areas of the model that could be improved to increase the prediction accuracy and model applicability. The polarization models used in this work are rather simple. Vertical polarization (TM) was assumed for interactions with the floor and ceiling, and horizontal polarization (TE) was assumed for interactions with vertical walls. While this first approximation has been used for the results presented here, this model does not account for depolarization upon reflection and transmission. Several researchers have studied the effects of polarization in buildings [Ber86], [Cox86], [Haw91], [Vil91], [Ho92], [McG92]. The inclusion of a larger amount of polarization dependence would likely increase the applicability and accuracy of the model.

This work has assumed homogeneous, infinitely thin, smooth planar surfaces infinite in extent for the development of the reflection and transmission coefficient models. Some improvements to these assumptions include phase effects due to wall thickness and cavities inside cinderblock walls, and models for the scattering from non-homogeneous walls such as drywall mounted on metal studs. Incorporation of these effects based on theoretical models may allow these constraints to be relaxed.

The uniform geometrical theory of diffraction model used here can only be used for single-diffraction paths. Multiple diffraction and reflection-diffraction are not included in the model. An expansion of the diffraction model could lead to more accurate prediction. A double wedge diffraction coefficient model is given in [Sch91].

Many of the procedures in the propagation prediction were performed manually. Full automation of the prediction process from building blueprint entry and transmitter 
and receiver placement to ray tracing and data processing would facilitate the use of this program as a tool for wireless communication system installation and design. Unmodeled objects such as furniture have not been included. Incorporation of additional site-specific information with the appropriate propagation models will likely increase the prediction accuracy.

Another critical aspect in the utility of this method is the computational complexity which leads to large execution times. Depending on the complexity of the building blueprint, the receiver thresholds, the tessellation frequency, and the efficiency of the bounding volume structure, the ray tracing can take anywhere from several hours to several days to complete. An automated bounding volume generation algorithm, an adaptable tessellation frequency, and an adaptable threshold could be used to decrease execution time. One way to circumvent the large run times would be to implement parallel processing where different rays could be traced on different machines. The raw output files could then be combined and processed in the same manner as the raw ray tracing output files described here. 


\section{References}

[Ale83a] S.E. Alexander, "900 MHz Propagation Within Buildings," IEE 2nd Intl. Conf. on Radio Spectrum Conservation Techniques, Birmingham, UK, 6-8 Sept. 1983, pp. 51-55.

[Ale83b] S.E. Alexander, "Characterising Buildings for Propagation at $900 \mathrm{MHz}$," Electronics Letters, Vol. 19, No. 29, September 29, 1983, p. 860.

[Ame53] W.S. Ament, "Toward a Theory of Reflection by a Rough Surface," Proceedings of the IRE, Vol. 41, No. 1, January 1953, pp. 142-146.

[Arn89] H.W. Arnold, R.R. Murray, and D.C. Cox, "815 MHz Radio Attenuation Measured Within Two Commercial Buildings," IEEE Transactions on Antennas and Propagation, Vol. 37, No. 10, October 1989, pp. 1335-1339

[Ba189] C.A. Balanis, Advanced Engineering Electromagnetics, John Wiley \& Sons, New York, 1989.

[Bec63] P. Beckmann and A. Spizzichino, The Scattering of Electromagnetic Waves from Rough Surfaces, Pergamon Press, Oxford, England, 1963.

[Bec68] P. Beckmann, The Depolarization of Electromagnetic Waves, Golem Press, Boulder, Colorado, 1968.

[Bel63] P.A. Bello and B.D. Nelin, "The Effect of Frequency Selective Fading on the Binary Error Probabilities of Incoherent and Differentially Coherent Matched Filter Receivers," IEEE Transactions on Communications Systems, Jun. 1963, Vol. CS-11, Dec. 1963, pp. 170-186.

[Ber86] S.A. Bergmann and H.W. Arnold, "Polarisation Diversity in Portable Communications Environment," Electronics Letters, Vol. 22, No. 11, May 22, 1986, pp. 609-610.

[Ber89] R.C. Bernhardt, "The Effect of Path Loss Models on the Simulated Performance of Portable Radio Systems," IEEE Globecom '89, Dallas, TX, pp. 1356-1360.

[Bis88] B. Bisceglia, G. Franceschetti, G. Mazzarella, I.M. Pinto, and C. Savarese, "Symbolic Code Approach to GTD Ray Tracing," IEEE Transactions on Antennas and Propagation, Vol. 36, No. 10, October 1988, pp. 1492-1495.

[Bor65] M.Born and E. Wolf, Principles of Optics, Pergammon Press, New York, 1965. 
[Bow69] J.J. Bowman, T.B.A. Senior, and P.L.E. Uslenghi, Eds., Electromagnetic and Acoustic Scattering by Simple Shapes, Elsevier Publishing Co., New York, 1969.

[Bro77] G.S. Brown, "The Average Impulse Response of a Rough Surface and Its Applications," IEEE Transactions on Antennas and Propagation, Vol. AP-25, No. 1, Jan. 1977, pp. 67-74.

[Bul87] R.J.C. Bultitude, "Measurement, Characterization and Modeling of Indoor 800/ $900 \mathrm{MHz}$ Radio Channels for Digital Communications," IEEE Communications Magazine, Vol. 25, No. 6, June 1987, pp. 5-12.

[Bul89] R.J.C. Bultitude, S.A. Mahmoud, and W.A. Sullivan, "A Comparison of Indoor Radio Propagation Characteristics at $910 \mathrm{MHz}$ and $1.75 \mathrm{GHz}$," IEEE Journal on Selected Areas in Communications, Vol. 7, No. 1, January 1989.

[Bur83] W.D. Burnside, "High Frequency Scattering by a Thin Lossless Dielectric Slab," IEEE Transactions on Antennas and Propagation, Vol. AP-31, No. 1, Jan. 1983, pp. 104-110.

[Che83] D.K. Cheng, Field and Wave Electromagnetics, Addison-Wesley, 1983.

[Chi89] S.T.S. Chia and A. Baran, "900 MHz Attenuation and Bit Error Ratio Measurements Inside a Modern Office Building," IEE 5th Intl. Conf. on Mobile Radio and Personal Communications, Warwick, UK, 11-14 Dec. 1989, pp. 33-37.

[Chu87a] J.C-I. Chuang, "The Effects of Time Delay Spread on Portable Radio Communications Channels with Digital Modulation," IEEE Journal on Selected Areas in Communications, Vol. SAC-5, No. 5, Jun. 1987, pp. 879-889.

[Chu87b]J.C-I. Chuang, "The Effects of Multipath Delay Spread on Timing Recovery," IEEE Transaction on Vehicular Technology, Vol. VT-35, No. 3, Aug. 1987, pp. 135-140.

[Col85] R.E. Collin, Antennas and Radiowave Propagation, McGraw-Hill, 1985.

[Cou90] L.W. Couch II, Digital and Analog Communication Systems, Macmillan, New York, 1990.

[Cox73] D.C. Cox, "910 MHz Urban Mobile Radio Propagation: Multipath Characteristics in New York City," IEEE Transactions on Communications, Vol. COM-21, No. 11, Nov. 1973, pp. 1188-1194. 
[Cox84] D.C. Cox, R.R. Murray, and A.W. Norris, "800 MHz Attenuation Measured In and Around Suburban Houses," AT\&T Bell Laboratories Technical Journal, Vol. 63, July/August, 1984, pp. 921-954.

[Cox85] D.C. Cox, R.R. Murray, and A.W. Norris, "Antenna Height Dependence of 800 $\mathrm{MHz}$ Attenuation Measured in Houses," IEEE Transactions on Vehicular Technology, Vol. VT-34, No. 2, May 1985, pp. 108-115.

[Cox86] D.C. Cox, R.R. Murray, H.W. Arnold, A.W. Norris, and M.F. Wazowicz, “CrossPolarization Coupling Measured for $800 \mathrm{MHz}$ Radio Transmission In and Around Houses and Large Buildings," IEEE Transactions on Antennas and Propagation, Vol. AP-34, No. 1, Jan. 1986, pp. 83-87.

[Des72] G.A. Deschamps, "Ray Techniques in Electromagnetics," Proceedings of the IEEE, Vol. 60, No. 9, Sep. 1972, pp. 1022-1035.

[Dev86] D.M.J. Devasirvatham, "Time Delay Spread and Signal Level Measurements of $850 \mathrm{MHz}$ Radio Waves in Building Environments," IEEE Transactions on Antennas and Propagation, Vol. AP-34, No. 11, Nov. 1986, pp. 1300-1305.

[Dev87a] D.M.J. Devasirvatham, "A Comparison of Time Delay Spread and Signal Level Measurements Within Two Dissimilar Office Buildings," IEEE Transactions on Antennas and Propagation, Vol. AP-35, No. 3, March 1987, pp. 319-324.

[Dev87b]D.M.J. Devasirvatham, "Multipath Time Delay Jitter Measured at $850 \mathrm{MHz}$ in the Portable Radio Environment," IEEE Journal on Selected Areas in Communications, Vol. SAC-5, No. 5, Jun. 1987, pp. 855-861.

[Dev87c] D.M.J. Devasirvatham "Multipath Time Delay Spread in the Digital Portable Radio Environment," IEEE Communications Magazine, Vol. 25, No. 6, June 1987, pp. 13-21.

[Dev89] D.M.J. Devasirvatham, R.R. Murray, and C. Banerjee, "Time Delay Spread Measurements at $850 \mathrm{MHz}$ and $1.7 \mathrm{GHz}$ Inside a Metropolitan Office Building," Electronics Letters, Vol. 25, No. 3, February 2, 1989, pp. 194-195.

[Dev90a] D.M.J. Devasirvatham, M.J. Krain, and D.A. Rappaport, "Radio Propagation Measurements at $850 \mathrm{MHz}, 1.7 \mathrm{GHz}$, and $4.0 \mathrm{GHz}$ inside two dissimilar office buildings," Electronics Letters, Vol. 26, no. 7, 1990, pp. 445-447.

[Dev90b]D.M.J. Devasirvatham, C. Banerjee, M.J. Krain, and D.A. Rappaport, "MultiFrequency Radiowave Propagation Measurements in The Portable Environment," IEEE ICC'90, Atlanta, GA, April 16-19, 1990. 
[Dev91a]D.M.J. Devasirvatham, "Multi-Frequency Propagation Measurements and Models in a Large Metropolitan Commercial Building for Personal Communications," IEEE Symposium on Personal Indoor and Mobile Communication, London, September 1991, pp. 98-103.

[Dev91b]D.M.J. Devasirvatham, C. Banerjee, R.R. Murray, and D.A. Rappaport, "FourFrequency Radiowave Propagation Measurements of the Indoor Environment in a Large Metropolitan Commercial Building," IEEE Globecom '91, Phoenix, AZ, December 1991, pp. 1282-1286.

[deW88] J.P. deWeck, P. Merki, and R. Lorenz, "Power Delay Profiles Measured in Mountainous Terrain, " $38^{\text {th }}$ IEEE Vehicular Technology Conference, June 15, 1988, Philadelphia, PA, pp. 105-111.

[Dix83] W.J. Dixon and F.J. Massey, Jr., Introduction to Statistical Analysis, McGrawHill, New York, 1983.

[Dri91] P.F. Driessen, "Development of a Propagation Model in the $20-60 \mathrm{GHz}$ Band for Wireless Indoor Communications," IEEE Pacific Rim Conference, Victoria, B.C., Canada, May 1991, pp. 59-62.

[Dri92] P.F. Driessen, et. al., "Ray Model of Indoor Propagation," in proceedings of 2nd Annual Virginia Tech Symposium on Wireless Personal Communications, Blacksburg, VA, June 17-19, 1992, pp. 17-1 through 17-12.

[Gan72] M.J. Gans, "A Power-Spectral Theory of Propagation in the Mobile-Radio Environment," IEEE Transactions on Vehicular Technology, Vol. VT-21, No. 1, Feb. 1972, pp. 27-38.

[Gan89] R. Ganesh and K. Pahlavan, "On the Modeling of Fading Multipath Indoor Radio Channels," IEEE Globecom '89, Dallas, TX, November 1989, pp. 13461350.

[Gan90] R. Ganesh and K. Pahlavan, "Effects of Traffic and Local Movements on Multipath Characteristics of an Indoor Radio Channel," Electronics Letters, Vol. 26, No. 12, June 7, 1990, pp. 810-812.

[Gan91] R. Ganesh and K. Pahlavan, "Statistics of Short Time Variations of Indoor Radio Propagation,” IEEE ICC ‘91, Denver, CO, June 1991, pp. 1-5.

[Gla89] A.S. Glassner, Ed., An Introduction to Ray Tracing, Academic Press, New York, 1989.

[Gri87] J. Griffiths, Radio Wave Propagation and Antennas, Prentice-Hall, London, 1987. 
[Har61] R.F. Harrington, Time-Harmonic Electromagnetic Fields, McGraw-Hill, New York, 1961.

[Haw90] D.A. Hawbaker, and T.S. Rappaport, "Indoor Wide band Radiowave Propagation Measurements at $1.3 \mathrm{GHz}$ and $4.0 \mathrm{GHz}$," Electronics Letters, Vol. 26, No. 21 , October 11, 1990, pp. 1800-1802.

[Haw91] D.A. Hawbaker, "Indoor Wide Band Radio Wave Propagation Measurements and Models at $1.3 \mathrm{GHz}$ and $4.0 \mathrm{GHz}$," Master's Thesis in Electrical Engineering, Virginia Polytechnic Institute and State University, May 15, 1991.

[Ho92] C.M.P. Ho and T.S. Rappaport, "Effects of Antenna Polarization and Beam Pattern on Multipath Delay Spread and Path Loss in Indoor Obstructed Wireless Channels," First International Conference on Universal Personal Communications, Dallas, TX, September 29-October2, 1992, pp. 92-96.

[Hon91] W. Honcharenko and H. L. Bertoni, "UHF Propagation in modern office buildings," poster presented at First Virginia Tech Symposium on Wireless Personal Communications, Blacksburg, VA, June 5, 1991.

[Hon92] W. Honcharenko, H.L. Bertoni, J. Dailing, J. Qian, and H.D. Yee, "Mechanisms Governing UHF Propagation on Single Floors in Modern Office Buildings," accepted to appear in IEEE Transactions on Vehicular Technology, 1992.

[Hor86] J. Horikoshi, K. Tanaka, and T. Morinaga, "1.2 GHz Band Wave Propagation Measurements in Concrete Building for Indoor Radio Communications," IEEE Transactions on Vehicular Technology, Vol. VT-35, No. 4, November 1986, pp. 146-152.

[Ike91] F. Ikegami, T. Takeuchi, and S. Yoshida., "Theoretical prediction of mean field strength for urban mobile radio," IEEE Transactions on Antennas and Propagation, Vol. 39, pp. 299-302, Mar. 1991.

[Jak74] W. Jakes, Jr., ed., Microwave Mobile Communications, Wiley, New York, 1974.

[Kel57] J.B. Keller, "Diffraction by an Aperture," Journal of Applied Physics, vol. 28, April 1957, pp. 426-444.

[Kel62] J.B. Keller, "Geometrical Theory of Diffraction," Journal of the Optical Society of America, Vol. 52, No. 2, Feb. 1962, pp. 116-130.

[Ken76] H. Kenner, Geodesic Math and How to Use It, University of California Press, Berkeley, 1976. 
[Kia92] J. Kiang, "Geometrical Ray Tracing Approach for Indoor Wave Propagation in a Corridor," First International Conference on Universal Personal Communications, Dallas, TX, September 29-October 2, 1992, pp. 106-111.

[Kno85] E.F. Knott, et. al., Radar Cross Section - Its Prediction, Measurement, and Reduction, Artech House, Dedham, MA, 1985.

[Kon90] J.A. Kong, Electromagnetic Wave Theory, Wiley, New York, 1990.

[Kou74] R.G. Kouyoumjian and P.H. Pathak, "A Uniform Geometrical Theory of Diffraction for an Edge in a Perfectly Conducting Surface," Proceedings of the IEEE, vol. 62, Nov. 1974, pp. 1448-1461.

[Laf90] J-F. Lafortune, and M. Lecours, "Measurement and Modeling of Propagation Losses in a Building at $900 \mathrm{MHz}$, , IEEE Transactions on Vehicular Technology, Vol. 39, No. 2, May 1990, pp. 101-108.

[Lan92] O. Landron, "Microwave Multipath Resolution in Microcellular Environments," Master's Thesis in Electrical Engineering, Virginia Polytechnic Institute and State University, Blacksburg, VA, August 1992.

[Lau92] D.I.Laurenson, A.U.H. Sheikh, and S. McLaughlin, "Characterisation of the Indoor Mobile Radio Channel Using a Ray Tracing Technique," 1992 IEEE International Conference on Selected Topics in Wireless Communications, Vancouver, B.C., Canada, June 1992, pp. 65-68.

[Law92] M.C. Lawton and J.P. McGeehan, "A Deterministic Ray Launching Algorithm for the Prediction of Radio Channel Characteristics in Small Cell Environments," submitted to IEEE Transactions on Vehicular Technology, July 1992.

[Lee82] W.C.Y. Lee, Mobile Communications Engineering, McGraw-Hill, New York, 1982.

[Lee89] W.C.Y. Lee, Mobile Cellular Telecommunications Systems, McGraw-Hill, New York, 1989.

[Lia85] S.Y. Liao, Microwave Devices and Circuits, Prentice-Hall, Englewood Cliffs, NJ, 1985.

[Lib92] J.C. Liberti and T.S. Rappaport, "Statistics of Shadowing in Indoor Radio Channels at 900 and $1900 \mathrm{MHz}$," IEEE MILCOM '92, San Diego, CA, October 1992, pp. 1066-1070. 
[Lue84a] R.J. Luebbers, "Finite Conductivity Uniform GTD Versus Knife Edge Diffraction in Prediction of Propagation Path Loss," IEEE Transactions on Antennas and Propagation, Vol. AP-32, No. 1, January 1984, pp. 70-76.

[Lue84b] R.J. Luebbers, "Propagation Prediction for Hilly Terrain Using GTD Wedge Diffraction," IEEE Transactions on Antennas and Propagation, Vol. AP-32, No. 9, September 1984, pp. 951-955.

[Lui90] J.T-K. Lui, "Narrow Band Microwave Propagation Measurement System for Indoor Communication," Undergraduate Thesis, MPRG-TR-90-2, May 1990.

[McG92] W.A. McGladdery and S. Stapleton, "Investigation of Polarization Effects in Indoor Radio Propagation," 1992 IEEE International Conference on Selected Topics in Wireless Communications, Vancouver, B.C., Canada, June 1992, pp. 53-56.

[McK91] J.W. McKown and R.L. Hamilton, "Ray Tracing as a Design Tool for Radio Networks," IEEE Network Magazine, Vol. 5, No. 6, Nov. 1991, pp. 27-30.

[Mic92] Microcell Report, Vol. 3, No. 9, September 1992.

[Mo191] D. Molkdar, "Review on radio propagation into and within buildings," IEE Proceedings- $H$, Vol. 138, No. 1, February 1991, pp. 61-73.

[Mor92] G. Morrison, M. Fattouche, and H. Zaghloul, "Statistical Analysis and Autoregressive Modeling of the Indoor Radio Propagation Channel," First International Conference on Universal Personal Communications, Dallas, TX, September 29-October 2, 1992, pp. 97-101.

[Mot88] A.J. Motley and J.M.P. Keenan, "Personal Communication Radio Coverage in Buildings at $900 \mathrm{MHz}$ and $1700 \mathrm{MHz}$," Electronics Letters, Vol. 24, No. 12, 1988, pp. 763-764.

[Mot90] A.J. Motley, and J.M. Keenan, "Radio coverage in buildings," British Telecom Technology Journal, Special Issue on Mobile Communications, Vol. 8, No. 1, Jan. 1990, pp. 19-24.

[Noe86] A.R. Noerpel, "Use of Physical Optics to Characterize Building Reflections," IEEE ICC '86, Toronto, Canada, June 1986, pp. 847-851.

[Ogi91] J.A. Ogilvy, Theory of Wave Scattering from Random Rough Surfaces, Adam Hilger, Philadelphia, 1991. 
[Owe89] F.C. Owen, and C.D. Pudney, "Radio Propagation for Digital Cordless Telephone at $1700 \mathrm{MHz}$ and $900 \mathrm{MHz}$," Electronics Letters, Vol. 25, No. 1, January 5,1989 , pp. 52-53.

[Pah89] K. Pahlavan, R. Ganesh, and T. Hotaling, "Multipath Propagation Measurements on Manufacturing Floors at $910 \mathrm{MHz}$," Electronics Letters, 2nd Feb., 1989, Vol. 25 , No. 3 , pp. $225-227$.

[Par89] J.D. Parsons, and J.G. Gardiner, Mobile Communication Systems, Blackie, London, 1989, pp. 72-75.

[Pat87] S.J. Patsiokas, B.K. Johnson, and J.L. Dailing, "The Effects of Buildings on the Propagation of Radio Frequency Signals," IEEE International. Communications Conference, Seattle, WA, June 1987, pp. 63-69.

[Pug76] A. Pugh, Polyhedra, A Visual Approach, University of California Press, Berkeley, 1976.

[Rae91] R.H. Raekken, H. Langaas, G. Lovnes, and S.E. Paulsen, "Wideband Impulse Response Measurements at $900 \mathrm{MHz}$ and $1.7 \mathrm{GHz}$," IEEE Globecom '91, Phoenix, AZ, December 1991, pp. 1303-1307.

[Rao82] S.M. Rao, D.R. Wilton, and A.W. Glisson, "Electromagnetic Scattering by Surfaces of Arbitrary Shape," IEEE Transactions on Antennas and Propagation, Vol. AP-30, No. 3, May 1982, pp. 409-418.

[Rap87] T.S. Rappaport, "Characterizing the UHF Factory Multipath Channel", Ph.D Dissertation in Electrical Engineering, Purdue University, Dec. 1987.

[Rap88a] T.S. Rappaport, “Wide-band Test Antennas,” RF Design, pp. 37-41, April 1988.

[Rap88b] T.S. Rappaport, "Delay Spread and Time Delay Jitter for the UHF Factory Multipath Channel," $38^{\text {th }}$ IEEE Vehicular Technology Conference, Philadelphia, May 1988, pp. 186-189.

[Rap89a] T.S. Rappaport, and C.D. McGillem, "UHF Fading in Factories," IEEE Journal on Selected Areas in Communications, Vol. 7, No. 1, January 1989, pp. 40-48.

[Rap89b] T.S. Rappaport, "Characterization of UHF Multipath Radio Channels in Factory Buildings," IEEE Transactions on Antennas and Propagation, Vol. 37, No. 8, August 1989, pp. 1058-1069.

[Rap90a] T.S. Rappaport, S.Y. Seidel, and R. Singh, "900-MHz Multipath Propagation Measurements for U.S. Digital Cellular Radiotelephone," IEEE Transactions on Vehicular Technology, Vol. 39, No. 2, May 1990, pp. 132-139. 
[Rap90b] T.S. Rappaport, S.Y. Seidel, "SIRCIM : Simulation of Indoor Radio Channel Impulse response Models,” 1990, VTIP, Inc.

[Rap90c] T.S. Rappaport, and L.B. Milstein, "Effects of Path Loss and Fringe User Distribution on CDMA Cellular Frequency Reuse Efficiency," IEEE Global Communications Conference, San Diego, CA, December 1990, pp. 500-506.

[Rap91a] T.S. Rappaport, S.Y. Seidel, and K. Takamizawa, "Statistical Channel Impulse Response Models for Factory and Open Plan Building Radio Communication System Design," IEEE Transactions on Communications, Vol. 39, No. 6, May 1991, pp. 794-897.

[Rap91b] T.S. Rappaport, "The Wireless Revolution," IEEE Communications Magazine, Vol. 29, No. 11, Nov. 1991, pp. 52-71.

[Rap91c] T.S. Rappaport and V. Fung, "Simulation of Bit Error Performance of FSK, BPSK, and $\pi / 4$ DQPSK in Flat Fading Indoor Radio Channels Using a Measurement-Based Channel Model," IEEE Transactions on Vehicular Technology, Vol. 40, No. 4, Nov. 1991 , pp. 731-740.

[Rap92] T.S. Rappaport and L.B. Milstein, "Effects of Radio Propagation Path Loss on DS-CDMA Cellular Frequency Reuse Efficiency for the Reverse Channel," IEEE Transactions on Vehicular Technology, Vol. 41, No. 3, August 1992, pp. 231-242.

[Ros66] R.A. Ross, "Radar Cross Section of Rectangular Flat Plates as a Function of Aspect Angle," IEEE Transactions on Antennas and Propagation, Vol. AP-14, May 1966, pp. 329-335.

[Ros91] J.P. Rossi, J.C. Bic, A.J. Levy, Y. Gabillet, and M. Rosen, "A Ray Launching Method for Radio-Mobile Propagation in Urban Area," IEEE Antennas and Propagation Symposium, London, Ontario, Canada, June 1991, pp. 1540-1543.

[Ros92] J.P. Rossi and A.J. Levy, "A Ray Model for Decimetric Radiowave Propagation in an Urban Area," Radio Science, Vol. 27, No. 6, Nov.-Dec. 1992, pp. 971-979.

[Ruc70] G.T. Ruck, ed., Radar Cross Section Handbook, Plenum Press, New York, 1970.

[Sal87] A.A.M. Saleh, and R.A. Valenzuela, "A Statistical Model for Indoor Multipath Propagation," IEEE Journal on Selected Areas in Communications, Vol. SAC-5, No. 2, February, 1987, pp. 128-137.

[Sch91] M. Schneider and R.J. Luebbers, “A General, Uniform Double Wedge Diffraction Coefficient," IEEE Transactions on Antennas and Propagation, Vol. 39, No. 1, January 1991, pp. 8-14. 
[Sch92] K.R. Schaubach, "Microcellular Radio Channel Prediction Using Ray Tracing," Master's Thesis in Electrical Engineering, Virginia Polytechnic Institute and State University, Blacksburg, VA August 1992.

[Sei89] S.Y. Seidel, "UHF Indoor Radio Channel Models for Manufacturing Environments," Masters Thesis in Electrical Engineering, Virginia Polytechnic Institute and State University, Blacksburg, VA, Aug. 1989.

[Sei91a] S.Y. Seidel and T.S. Rappaport, "900 MHz Path Loss Measurements and Prediction Techniques for In-Building Communication System Design," 41st IEEE Vehicular Technology Conference, St. Louis, MO, May 21, 1991, pp. 613-618.

[Sei91b] S.Y. Seidel, et.al, "Path Loss, Scattering, and Multipath Delay Statistics in Four European cities for Digital Cellular and Microcellular Radiotelephone," IEEE Transactions on Vehicular Technology, Vol. 40, No. 4, Nov. 1991, pp. 721-730.

[Sei92a] S.Y. Seidel and T.S. Rappaport, "914 MHz Path Loss Prediction Models for Wireless Communications in Multifloored Buildings," IEEE Transactions on Antennas and Propagation, Vol. 40, No. 2, Feb. 1992, pp. 1-11.

[Sei92b] S.Y. Seidel, "Site-Specific Propagation Prediction for Wireless In-Building Personal Communication System Design," report prepared for the Ph.D. Preliminary Examination, MPRG-TR-92-07, February 1992.

[Sei92c] S.Y. Seidel, et.al, "The Impact of Surrounding Buildings on Propagation for Wireless In-Building Personal Communications System Design", $42^{\text {nd }}$ IEEE Vehicular Technology Society Conference, Denver, CO, May 1992.

[Sie58] K.M. Siegel, "Far Field Scattering From Bodies of Revolution," Applied Science Research, sec. B, vol. 7, 1958, pp. 293-328.

[Sko70] M.I. Skolnik, ed., Radar Handbook, McGraw-Hill, New York, 1970.

[Sko80] M.I. Skolnik, Introduction to Radar Systems, McGraw-Hill, New York, 1980.

[Smi75] J.I. Smith, "A Computer Generated Multipath Fading Simulation for Mobile Radio," IEEE Transactions on Vehicular Technology, Vol. VT-24, No. 3, Aug. 1975, pp. 39-40.

[Som54] A.J.W. Sommerfeld, Optics, Academic Press, Inc., New York, 1954.

[Sou92] C.K. Sou, O. Landron, and M.J. Feuerstein, "Characterization of Electromagnetic Properties of Building Materials for use in Site-Specific Propagation Prediction," MPRG-TR-92-12, June 1992. 
[Ste90] R.T. Stevens, Fractal Programming and Ray Tracing with C++, M\&T Books, Redwood City, CA, 1990.

[Ste91] R. Steele, "The Importance of Propagation Phenomena in Personal Communication Networks," IEE Conference on Antennas and Propagation, York, UK, April 1991, pp. 1-5.

[Stu81] W.L. Stutzman and G.A. Thiele, Antenna Theory and Design, Wiley, New York, 1981.

[Tak91] T. Takeuchi, M. Sako, and S. Yoshida, "Multipath Delay Prediction on a Workstation for Urban Mobile Radio Environment," IEEE Globecom '91, Phoenix, AZ, December 1991, pp. 1308-1312.

[Te191] Telesis Technologies Laboratory, Experimental License Report to the FCC, Aug. 1991.

[Tho92] B. Thoma, T.S. Rappaport, and M.D. Keitz, "Simulation of Bit Error Performance and Outage Probability of p/4 DQPSK in Frequency-selective Indoor Radio Channels Using a Measurement-Based Channel Model," IEEE Globecom, Orlando, FL, December 1992, pp. 1825-1829.

[Tod92] S.R. Todd, M.S. El-Tanany, and S.A. Mahmoud, "Space and Frequency Diversity Measurements of the $1.7 \mathrm{GHz}$ Indoor Radio Channel for Wireless Personal Communications," First International Conference on Universal Personal Communications, Dallas, TX, September 29-October 2, 1992, pp. 87-91.

[Uri91] A. Urie, "Errors in Estimating Local Average Power of Multipath Signals," Electronics Letters, 14th February 1991, Vol. 27, No. 4, pp. 315-317.

[Van85] J. Van Bladel, Electromagnetic Fields, Hemisphere Publishing Corporation, New York, 1985.

[van87] J. van Rees, "Measurements of the Wide-Band Radio Channel Characteristics for Rural, Residential, and Suburban Areas," IEEE Trans. on Veh. Tech., Vol. VT-36, No. 1, Feb. 1987, pp. 2-6.

[Vil91] E.Vilar, "Depolarisation and Field Transmittances in Indoor Communications," Electronics Letters, Vol. 27, No. 9, April 25, 1991, pp. 732-733.

[Von54] A.R. Von Hippel, ed., Dielectric Materials and Applications, Publication of MIT Press, Cambridge, MA, 1954.

[Wen79] M.J. Wenninger, Spherical Models, Cambridge University Press, New York, 1979. 


\section{APPENDIX A: Fresnel Plane Wave Reflection Coefficients}

Consider a uniform plane wave with perpendicular (TE) polarization obliquely incident upon a planar dielectric boundary shown in Figure A. This derivation is paraphrased from [Che83]. The polarization is perpendicular because the $\vec{E}$ field is perpendicular to the plane of incidence. The plane of incidence is the plane that contains the vectors $\hat{k}_{i}$ and $\hat{n}$. Note that the entire region below the boundary is made up of the dielectric material and that the interface extends infinitely in the positive and negative $z$ directions. Table A summarizes the variables used to describe the fields.

\section{Perpendicular (TE) Polarization}

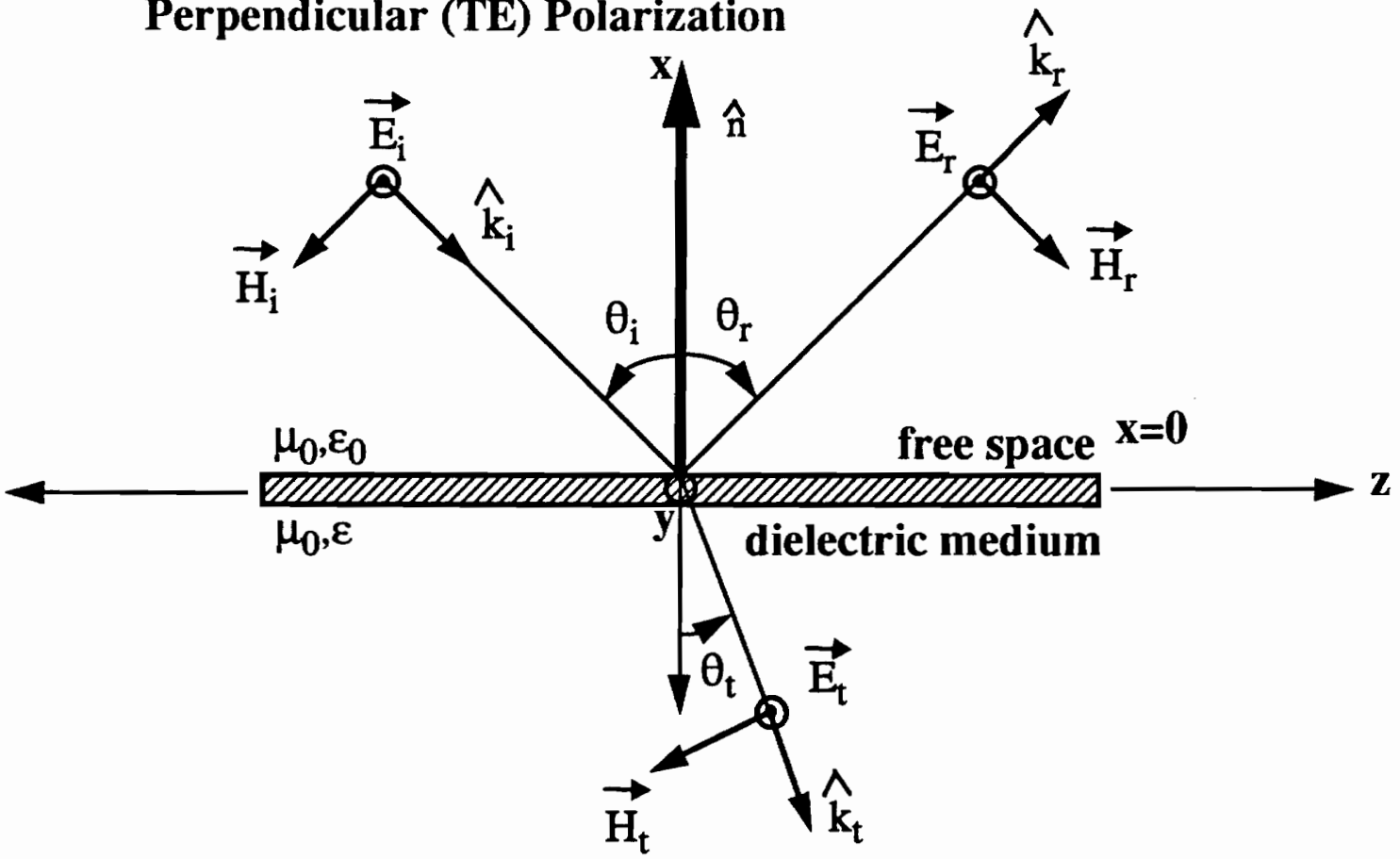

FIGURE A : Reflection and transmission field polarization of a perpendicularly (horizontally) polarized plane wave obliquely incident upon a planar dielectric boundary. Note that the entire region below the boundary is made up of the dielectric material and that the interface extends infinitely in the positive and negative $\mathrm{z}$ direction. 
TABLE A : Summary of variables used to describe plane wave reflection.

\begin{tabular}{|c|c|}
\hline Marioble & Bescription [initis] \\
\hline$\mu_{0}$ & Permeability of free space $[\mathrm{H} / \mathrm{m}]$ \\
\hline$\varepsilon_{0}$ & Permittivity of free space $[\mathrm{F} / \mathrm{m}]$ \\
\hline$\varepsilon=\varepsilon_{r} \varepsilon_{0}$ & Permittivity of dielectric material [F/m] \\
\hline$\sigma$ & Conductivity of dielectric material [S/m] \\
\hline$\varepsilon_{r}^{*}$ & Complex relative dielectric constant of material \\
\hline$\theta_{\mathrm{i}}$ & Angle of incidence (measured from surface normal) [radians] \\
\hline$\vec{E}_{i}$ & Incident electric field [V/m] \\
\hline$\vec{H}_{i}$ & Incident magnetic field $[\mathrm{A} / \mathrm{m}]$ \\
\hline$\hat{k}_{i}$ & Propagation direction of incident field \\
\hline$\theta_{\mathrm{r}}$ & Angle of reflection (measured from surface normal) [radians] \\
\hline$\vec{E}_{r}$ & Reflected electric field [V/m] \\
\hline$\vec{H}_{r}$ & Reflected magnetic field $[\mathrm{A} / \mathrm{m}]$ \\
\hline$\hat{k}_{r}$ & Propagation direction of reflected field \\
\hline$\theta_{1}$ & Angle of transmission (measured from surface normal) [radians] \\
\hline$\vec{E}_{t}$ & Transmitted electric field $[\mathrm{V} / \mathrm{m}]$ \\
\hline$\vec{H}_{t}$ & Transmitted magnetic field $[\mathrm{A} / \mathrm{m}]$ \\
\hline$\vec{k}_{t}$ & Propagation direction of transmitted field \\
\hline$\eta=\sqrt{\mu / \varepsilon}$ & Wave impedance in dielectric medium $[\Omega]$ \\
\hline$\eta_{0}$ & Wave impedance in free space $[\Omega]$ \\
\hline$k=2 \pi / \lambda=\omega \sqrt{\mu \varepsilon}$ & Wave number in dielectric medium [radians/m] \\
\hline$n_{2}=\sqrt{\varepsilon_{r}}$ & Index of refraction in dielectric medium \\
\hline$\hat{x}$ & Unit vector in the coordinate direction $x$ \\
\hline$\hat{y}$ & Unit vector in the coordinate direction $y$ \\
\hline
\end{tabular}




\begin{tabular}{|c|c|}
\hline Wariable & Description [units] \\
\hline$\hat{z}$ & Unit vector in the coordinate direction $\mathrm{z}$ \\
\hline$\Gamma_{\perp}$ & Reflection coefficient for perpendicular polarization \\
\hline$T_{\perp}$ & Transmission coefficient for perpendicular polarization \\
\hline$\Gamma_{\|}$ & Reflection coefficient for parallel polarization \\
\hline$T_{\|}$ & Transmission coefficient for parallel polarization \\
\hline
\end{tabular}

The incident electric and magnetic field phasors of the time-harmonic fields in the free space source-free region are [Che83]

$$
\begin{gathered}
\vec{E}_{i}(x, z)=\hat{y} E_{i o} e^{-j k_{0}\left(x \sin \theta_{i}+z \cos \theta_{i}\right)} \\
\vec{H}_{i}(x, z)=\frac{E_{i o}}{\eta}\left(-\hat{x} \cos \theta_{i}+\hat{z} \sin \theta_{i}\right) e^{-j k_{0}\left(x \sin \theta_{i}+z \cos \theta_{i}\right)}
\end{gathered}
$$

The reflected fields in the free space region are

$$
\begin{gathered}
\vec{E}_{r}(x, z)=\hat{y} E_{r o} e^{-j k_{0}\left(x \sin \theta_{r}-z \cos \theta_{r}\right)} \\
\vec{H}_{r}(x, z)=\frac{E_{r o}}{\eta}\left(\hat{x} \cos \theta_{r}+\hat{z} \sin \theta_{r}\right) e^{-j k_{0}\left(x \sin \theta_{r}-z \cos \theta_{r}\right)}
\end{gathered}
$$

In the dielectric region, the transmitted fields are represented by

$$
\begin{gathered}
\vec{E}_{t}(x, z)=\hat{y} E_{t o} e^{-j k\left(x \sin \theta_{t}+z \cos \theta_{t}\right)} \\
\vec{H}_{t}(x, z)=\frac{E_{t o}}{\eta}\left(-\hat{x} \cos \theta_{t}+\hat{z} \sin \theta_{t}\right) e^{-j k\left(x \sin \theta_{t}+z \cos \theta_{t}\right)}
\end{gathered}
$$

In order to satisfy Maxwell's equations, the tangential components of the $\vec{E}$ and $\vec{H}$ fields must be continuous at the boundary. Snell's laws of reflection and refraction give 


$$
\theta_{r}=\theta_{i} ; \frac{\sin \theta_{t}}{\sin \theta_{i}}=\frac{k_{0}}{k}=\frac{\omega \sqrt{\mu_{0} \varepsilon_{0}}}{\omega \sqrt{\mu_{0} \varepsilon}}=\frac{1}{\sqrt{\varepsilon_{r}}}=\frac{1}{n_{2}}
$$

Satisfaction of the boundary conditions on the surface require

$$
\begin{gathered}
E_{i o}+E_{r o}=E_{t o} \\
\frac{1}{\eta_{0}}\left(E_{i o}-E_{r o}\right) \cos \theta_{i}=\frac{E_{t o}}{\eta} \cos \theta_{t}
\end{gathered}
$$

Solving for the reflected and transmitted field amplitudes in terms of the incident field amplitudes gives the Fresnel reflection and transmission coefficients for perpendicular (TE) polarization.

$$
\begin{aligned}
& \Gamma_{\perp}=\frac{E_{\text {ro }}}{E_{i o}}=\frac{\left(\eta / \cos \theta_{t}\right)-\left(\eta_{0} / \cos \theta_{i}\right)}{\left(\eta / \cos \theta_{t}\right)+\left(\eta_{0} / \cos \theta_{i}\right)} \\
& T_{\perp}=\frac{E_{t o}}{E_{i o}}=\frac{2\left(\eta / \cos \theta_{t}\right)}{\left(\eta / \cos \theta_{t}\right)+\left(\eta_{0} / \cos \theta_{i}\right)}
\end{aligned}
$$

The reflection coefficient $\Gamma_{\perp}$ and the transmission coefficient $\tau_{\perp}$ are related as follows.

$$
1+\Gamma_{\perp}=T_{\perp}
$$

A similar analysis follows for parallel (TM) polarization shown in Figure B.

$$
\begin{gathered}
\Gamma_{\|}=\frac{E_{r o}}{E_{i o}}=\frac{\left(\eta \cos \theta_{t}-\eta_{0} \cos \theta_{i}\right)}{\left(\eta \cos \theta_{t}+\eta_{0} \cos \theta_{i}\right)} \\
T_{\|}=\frac{E_{t o}}{E_{i o}}=\frac{2 \eta \cos \theta_{i}}{\left(\eta \cos \theta_{t}+\eta_{0} \cos \theta_{i}\right)} \\
1+\Gamma_{\|}=T_{\|}\left(\frac{\cos \theta_{t}}{\cos \theta_{i}}\right)
\end{gathered}
$$




\section{Parallel (TM) Polarization}

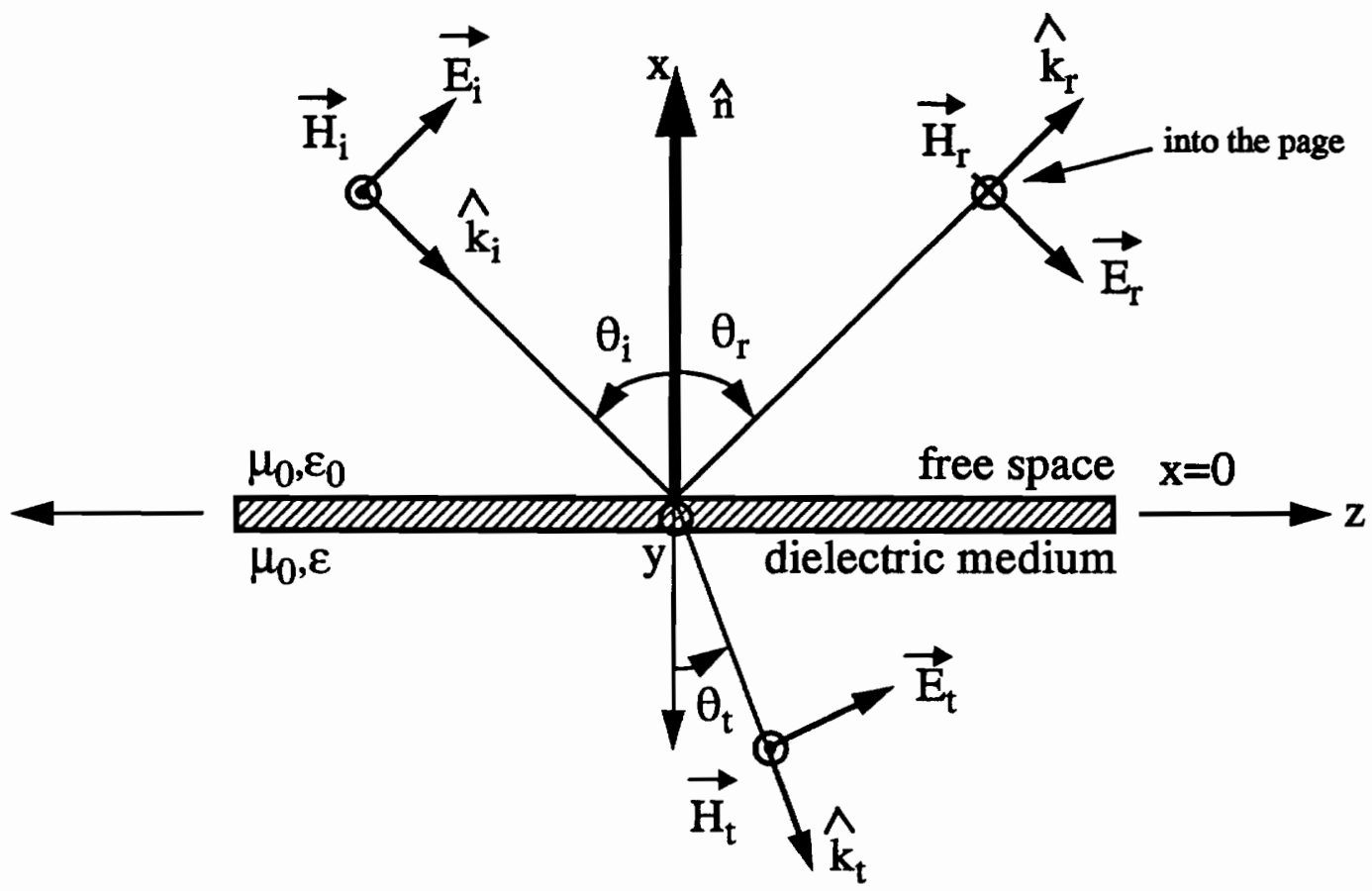

FIGURE B : Reflection and transmission field polarization of a vertically polarized plane wave incident upon a planar dielectric boundary. Note that the entire surface below the interface is made up of the dielectric medium and that the surface boundary extends to positive and negative infinity in the $\mathrm{z}$ direction.

Lossy materials may be considered by letting the relative permittivity of the dielectric constant be complex. The term $\frac{\sigma}{\omega \varepsilon}$ is referred to as the loss tangent.

$$
\varepsilon_{r}^{*}=\varepsilon_{r}\left(1-j \frac{\sigma}{\omega \varepsilon}\right)
$$

Polarizations other than vertical and horizontal may be divided into vertically and horizontally polarized components [Bec68]. Conductivity, permittivity, frequency, and angle of incidence influence the Fresnel reflection coefficients. 


\section{APPENDIX B: Physical Optics}

Consider a flat planar surface of area $a b$ as shown in Figure C. An electromagnetic

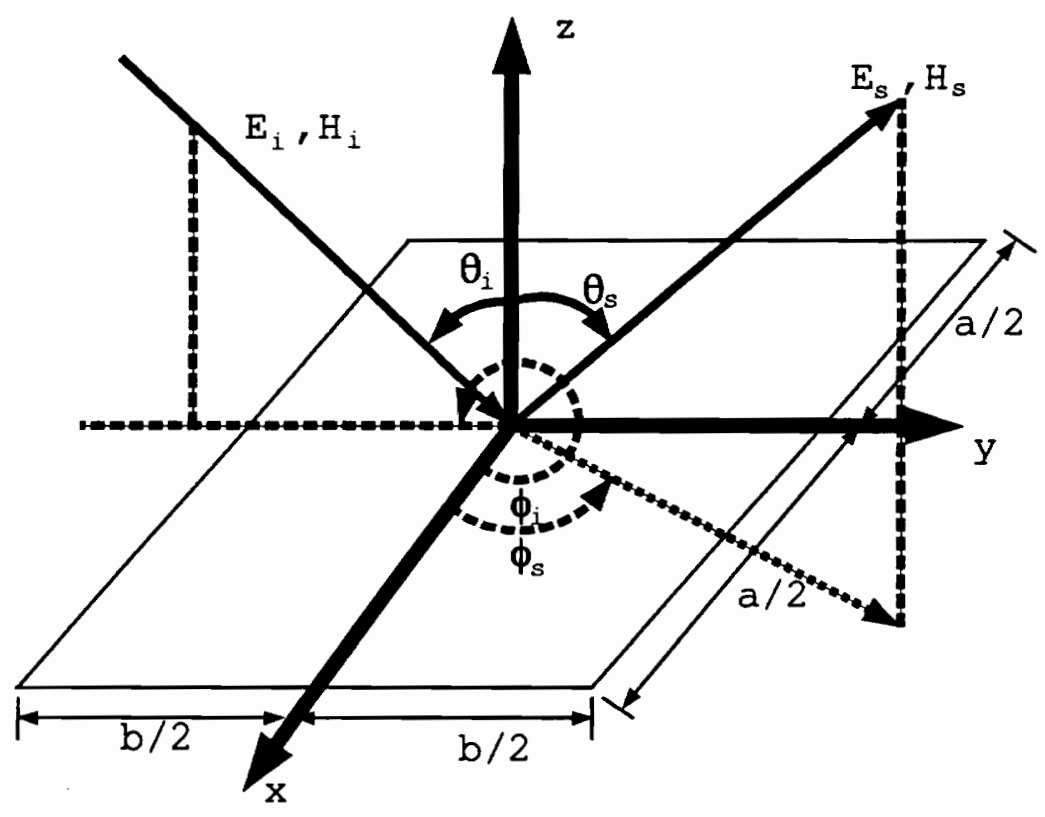

FIGURE C : Geometry for scattering by a perfectly conducting flat plate with area $a b$. An electromagnetic wave is incident at an angle $\theta_{i}$ from the surface normal, and $\phi_{\mathrm{i}}$ from the $\mathrm{x}$-axis as indicated in the figure.

wave is incident at the angles $\theta_{i}, \phi_{i}$ with respect to the center of the surface. This wave is TE with respect to the propagation direction. The polarization is shown in Figure D. Using the notation of [Bal89], the incident $\vec{E}$ and $\vec{H}$ fields are

$$
\begin{gathered}
\vec{E}_{i}=\left[-\hat{x} \sin \phi_{i}+\hat{y} \cos \phi_{i}\right] \frac{E_{0}}{r_{1}} e^{-j k r_{1}} \\
\vec{H}_{i}=\left[\hat{x} \cos \theta_{i} \cos \phi_{i}+\hat{y} \cos \theta_{i} \sin \phi_{i}-\hat{z} \sin \theta_{i}\right] \frac{E_{0}}{\eta r_{1}} e^{-j k r_{1}}
\end{gathered}
$$

where $E_{0}$ is the electric field strength at 1 meter and $r_{l}$ is the distance from the source to the exact location on the plate in three dimensions. For all calculations $E_{0}$ is the electric field strength for free space propagation at one meter from the source. Although the total 


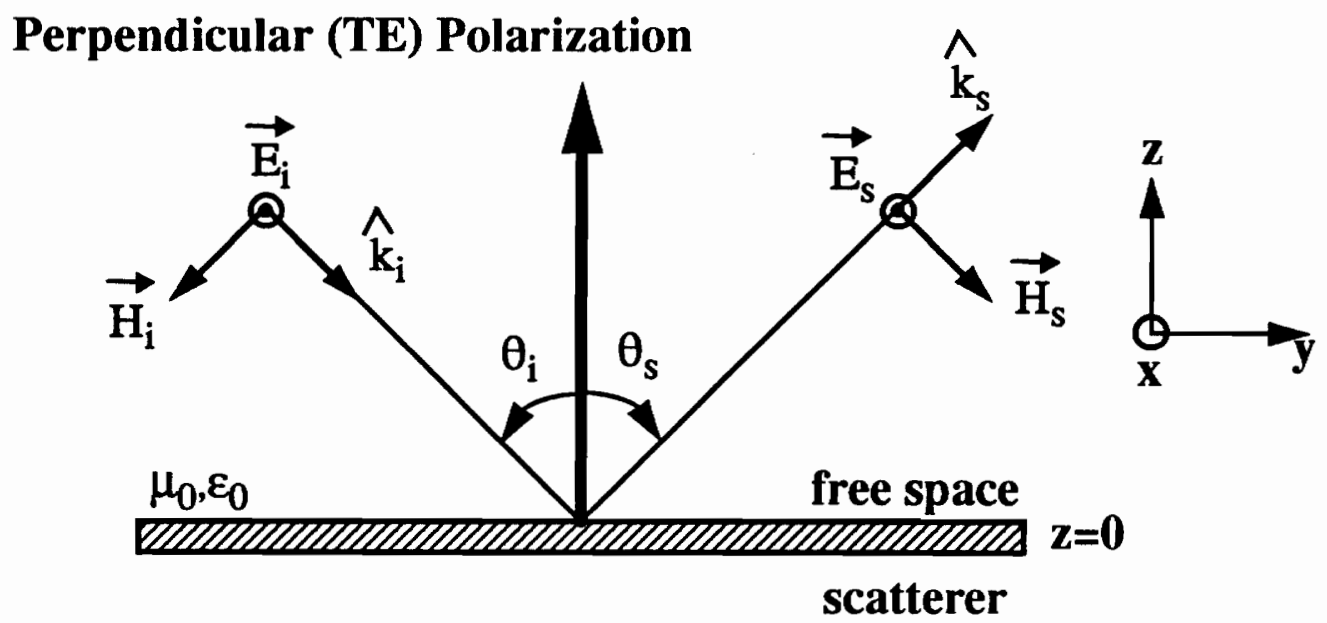

FIGURE D : Incident and scattered field polarization of a perpendicularly (TE) polarized plane wave incident upon a finite flat plate scatterer $\left(\phi_{i}=270, \phi_{s}=90\right)$.

field strength at one meter may not be equal to the free space value, the free space value is used as the reference field strength for all calculations. The equivalent problem is determined by using the appropriate surface currents to produce the correct scattered field everywhere due to the presence of the scatterer. The appropriate scattered fields are computed by free space radiation of the surface currents in the absence of the scatterer. Physical optics assumes that the current on the surface of the plate is proportional to the total field at the surface as determined by geometrical optics. For a dielectric surface scatterer and TE polarization, the induced electric and magnetic surface currents are proportional to the total tangential fields on the surface.

$$
\begin{gathered}
\vec{J}_{s} \cong \hat{n} \times\left.\vec{H}_{T}\right|_{x=x^{\prime}, y=y^{\prime}, z=0} \\
\vec{M}_{s} \cong-\hat{n} \times\left.\vec{E}_{T}\right|_{x=x^{\prime}, y=y^{\prime}, z=0}
\end{gathered}
$$

The total tangential fields are the sum of the incident and reflected fields on the surface of the scatterer. Hence, the surface currents become

$$
\vec{J}_{s} \cong \frac{E_{0}}{\eta r_{1}}\left(1-\Gamma_{\perp}\left(\dot{r}^{\prime}\right)\right)\left[\cos \theta_{i} \cos \phi_{i} \hat{y}-\cos \theta_{i} \sin \phi_{l} \hat{x}\right] e^{-j k r_{1}}
$$




$$
\vec{M}_{s} \cong \frac{E_{0}}{r_{1}}\left(1+\Gamma_{\perp}\left(\vec{r}^{\prime}\right)\right)\left[\sin \phi_{i} \hat{y}+\cos \phi_{i} \hat{x}\right] e^{-j k r_{1}}
$$

For a perfect electric conductor (PEC), $\Gamma_{\perp}=-1$ and the magnetic surface current is zero. If a dielectric scatterer is modeled, the Fresnel plane wave reflection coefficients on the surface of the scatterer are used in equations (21) and (22). These currents are exact for an infinite plane, but approximate for the scatterer in Figure $\mathrm{C}$, due to the neglect of the edges. These currents are the physical optics approximations for the true currents.

The scattered fields are determined by the radiation of the currents on the surface of the plate. The vector potentials are

$$
\begin{aligned}
& \vec{A}(\vec{r})=\frac{\mu}{4 \pi} \iint_{S^{\prime}} \vec{J}_{s}\left(\vec{r}^{\prime}\right) \frac{e^{-j k R}}{R} d s^{\prime} \\
& \vec{F}(\vec{r})=\frac{\varepsilon}{4 \pi} \iint_{S^{\prime}} \vec{M}_{s}\left(\vec{r}^{\prime}\right) \frac{e^{-j k R}}{R} d s^{\prime}
\end{aligned}
$$

where $R=|\vec{r}-\vec{r}|$ is the distance from a point on the surface to the observation point, and the integration is performed over the entire surface of the plate $S$ '. The total scattered electric field is the superposition of the electric field due to each of the vector potentials. According to [Bal89, p. 283], the magnetic field due to the vector potential $\vec{A}$ is

$$
\vec{H}_{A}(\vec{R})=-\frac{1}{4 \pi} \iint_{S^{\prime}}(\hat{R} \times \vec{J}) \frac{1+j k R}{R^{2}} e^{-j k R} d s^{\prime}
$$

where $\hat{R}$ is the unit vector in the direction of $\vec{R}$. The electric field due to the electric vector potential in the $\hat{x}$ direction can be written as [Bal89]

$$
E_{A_{x}}=-\frac{j \eta E_{0}}{4 \pi k} \iint_{S^{\prime}}\left\{G_{1} J_{x}+\left(x-x^{\prime}\right) G_{2} \times\left[\left(x-x^{\prime}\right) J_{x}+\left(y-y^{\prime}\right) J_{y}\right]\right\} e^{-j k R} d s^{\prime}
$$

with no approximations where

$$
G_{1}=\frac{-1-j k R+k^{2} R^{2}}{R^{3}} \text { and } G_{2}=\frac{3+j 3 k R-k^{2} R^{2}}{R^{5}}
$$


The electric field due to the magnetic vector potential $\vec{F}$ is

$$
E_{F_{x}}=-\frac{1}{4 \pi} \iint_{S^{\prime}}\left(z-z^{\prime}\right) M_{y}\left(\frac{1+j k R}{R^{3}}\right) e^{-j k R} d s^{\prime}
$$

and the total electric field which is polarized in the $\hat{x}$ direction is $E_{T_{x}}=E_{A_{x}}+E_{F_{x}}$. The electric field in the other directions are zero, and the magnetic fields can be determined by equations similar to (26) and (28) that can be found in [Bal89].

Equations (26) and (28) are the exact expressions for the scattered field due to a surface current and these expressions are valid in the near field. Its accuracy is determined by the accuracy of the physical optics assumption for the electric and magnetic currents on the surface of the plate in equations (21) and (22). 


\section{APPENDIX C: Measured and Predicted Power Delay Profiles}

Whittemore Hall 1.3 GHz - 9 locations

Whittemore Hall 4.0 GHz - 21 locations

Norris Hall 4.0 GHz - 15 locations 

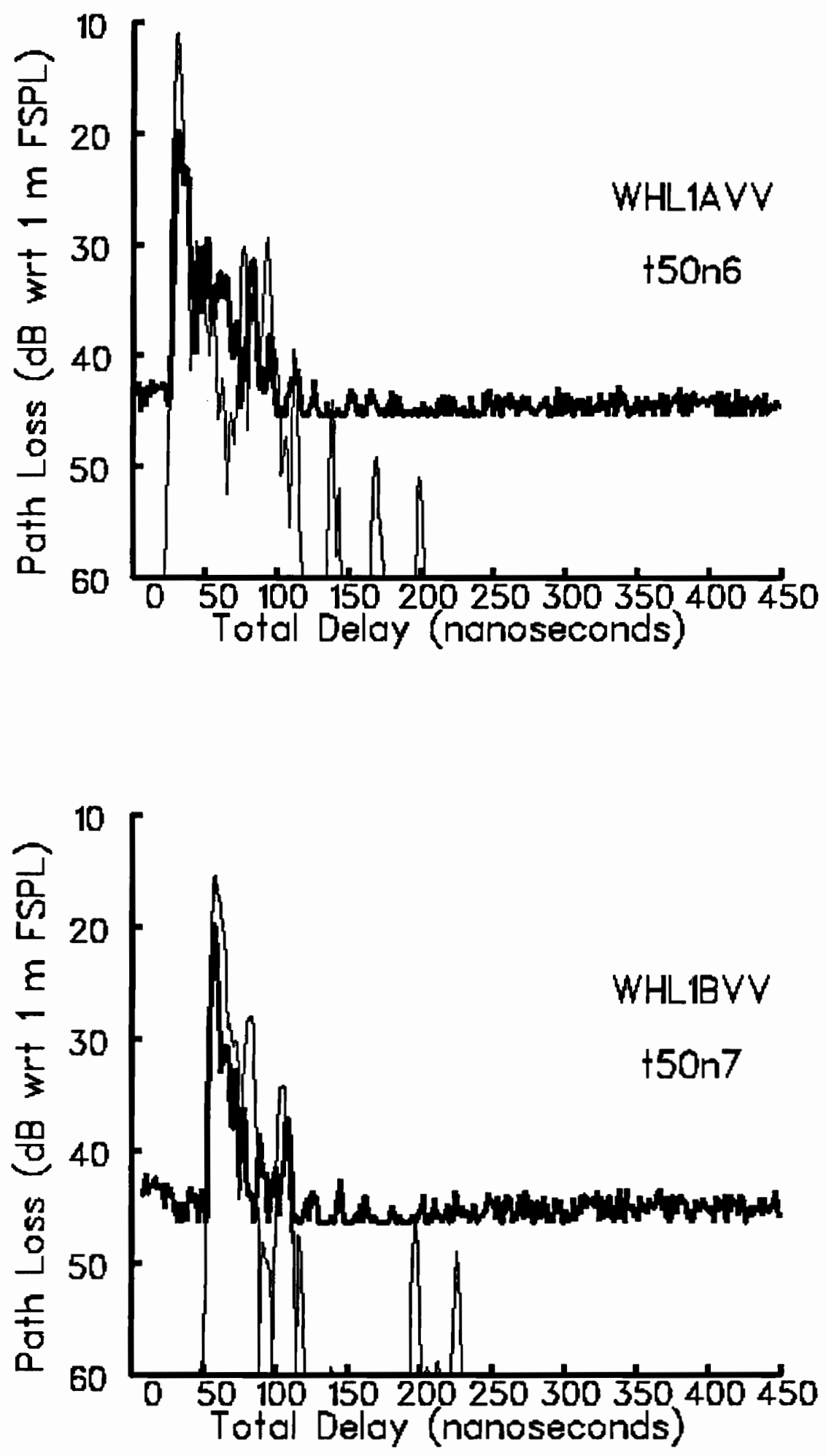

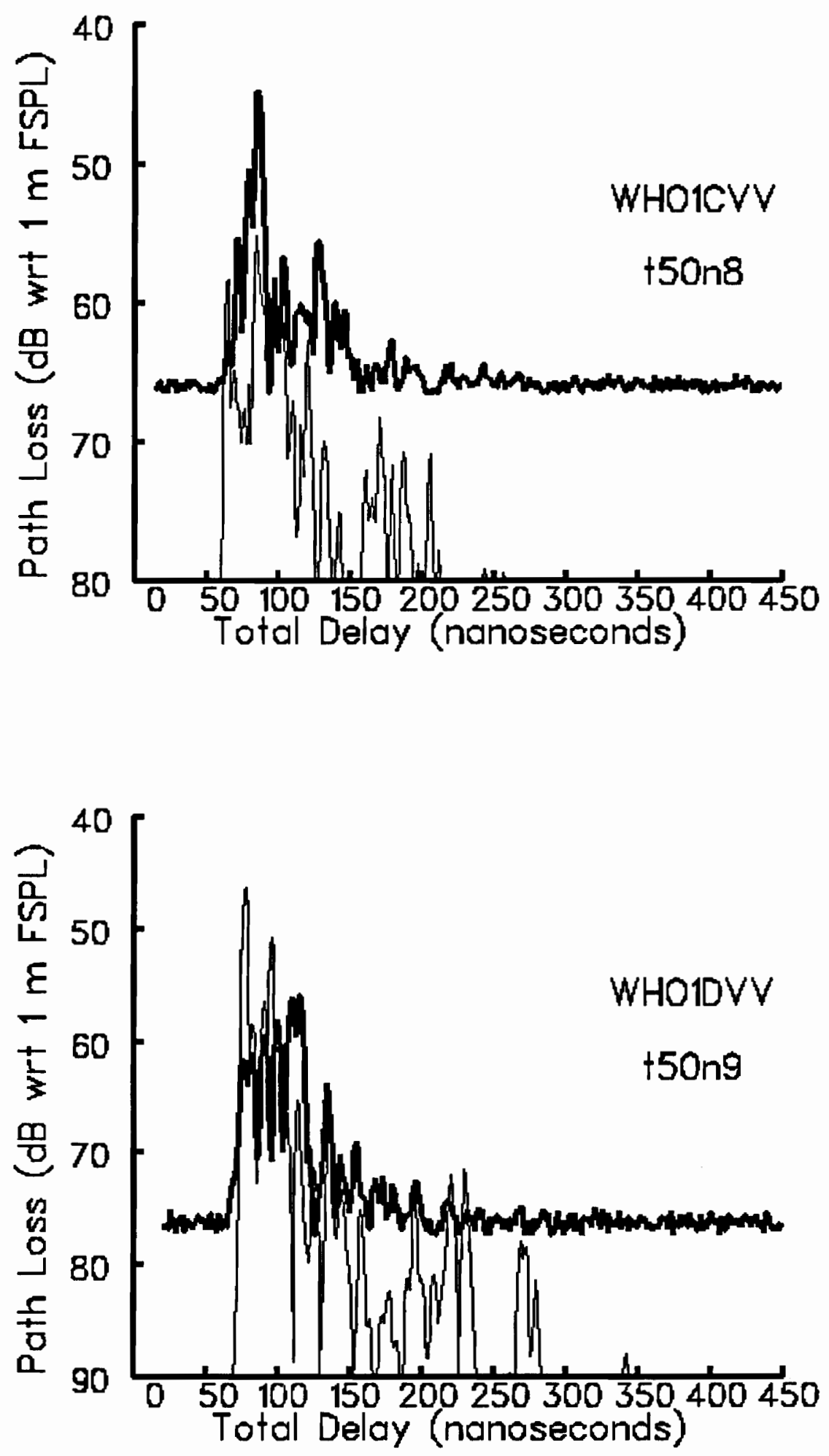

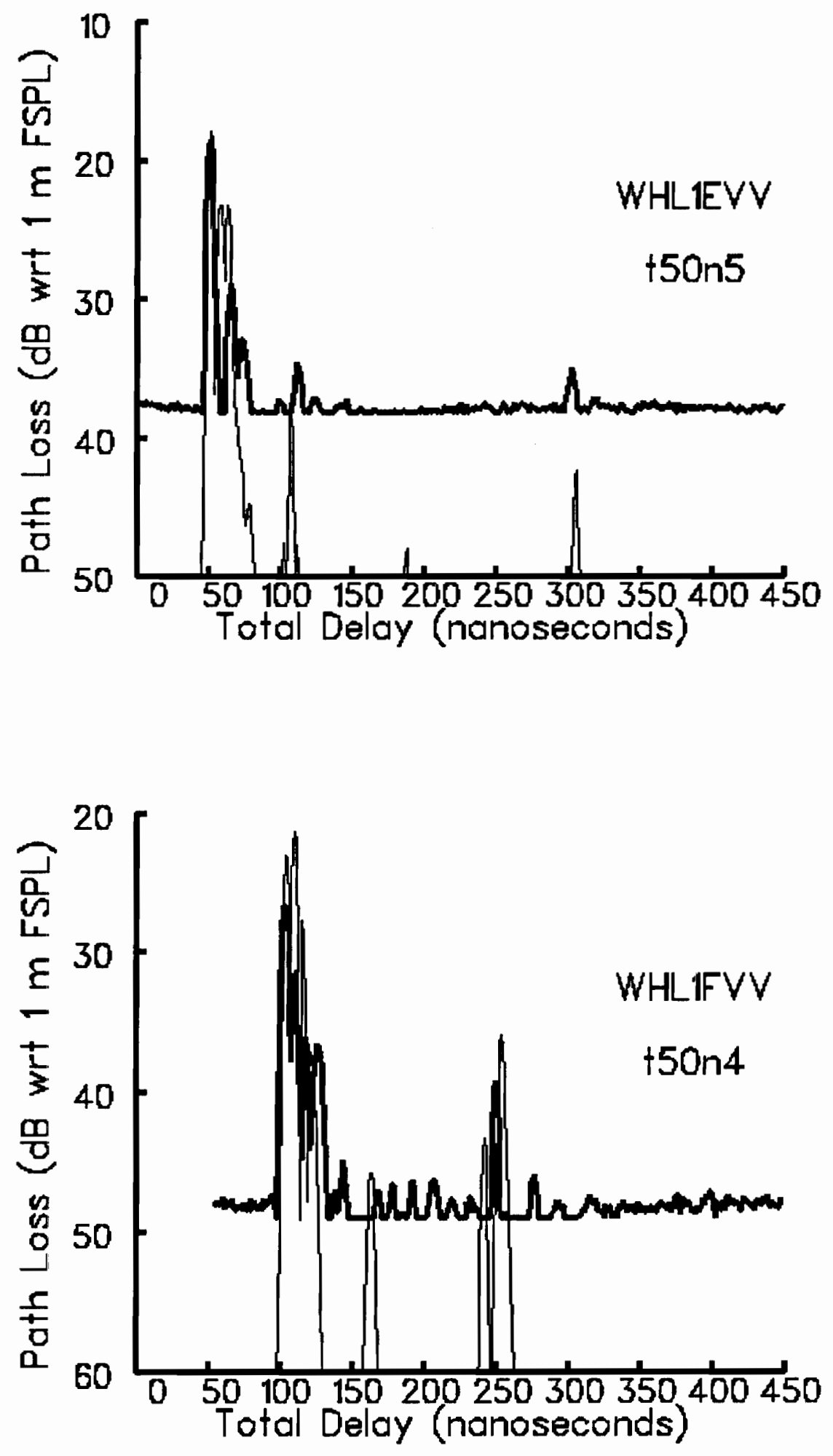

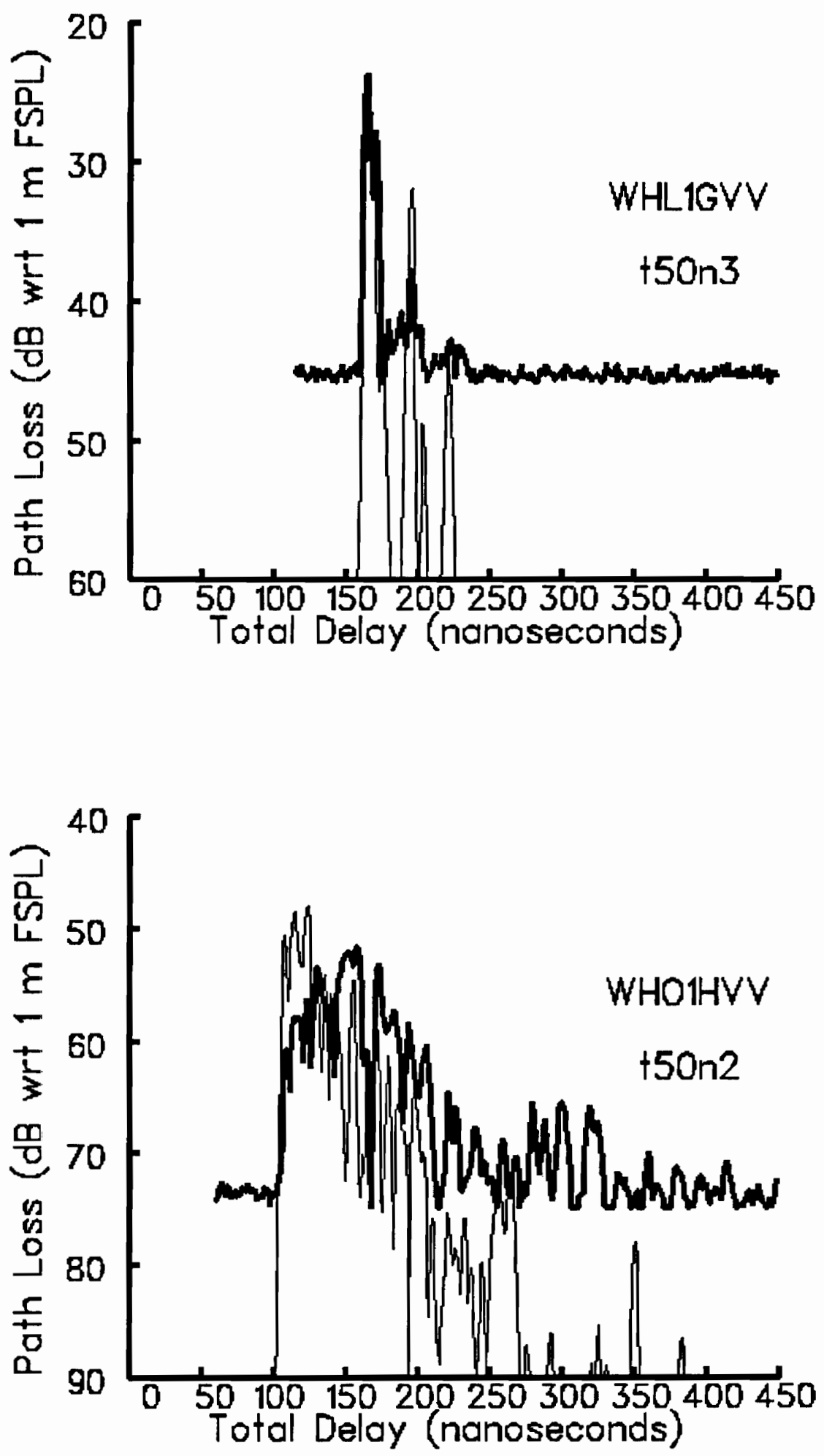


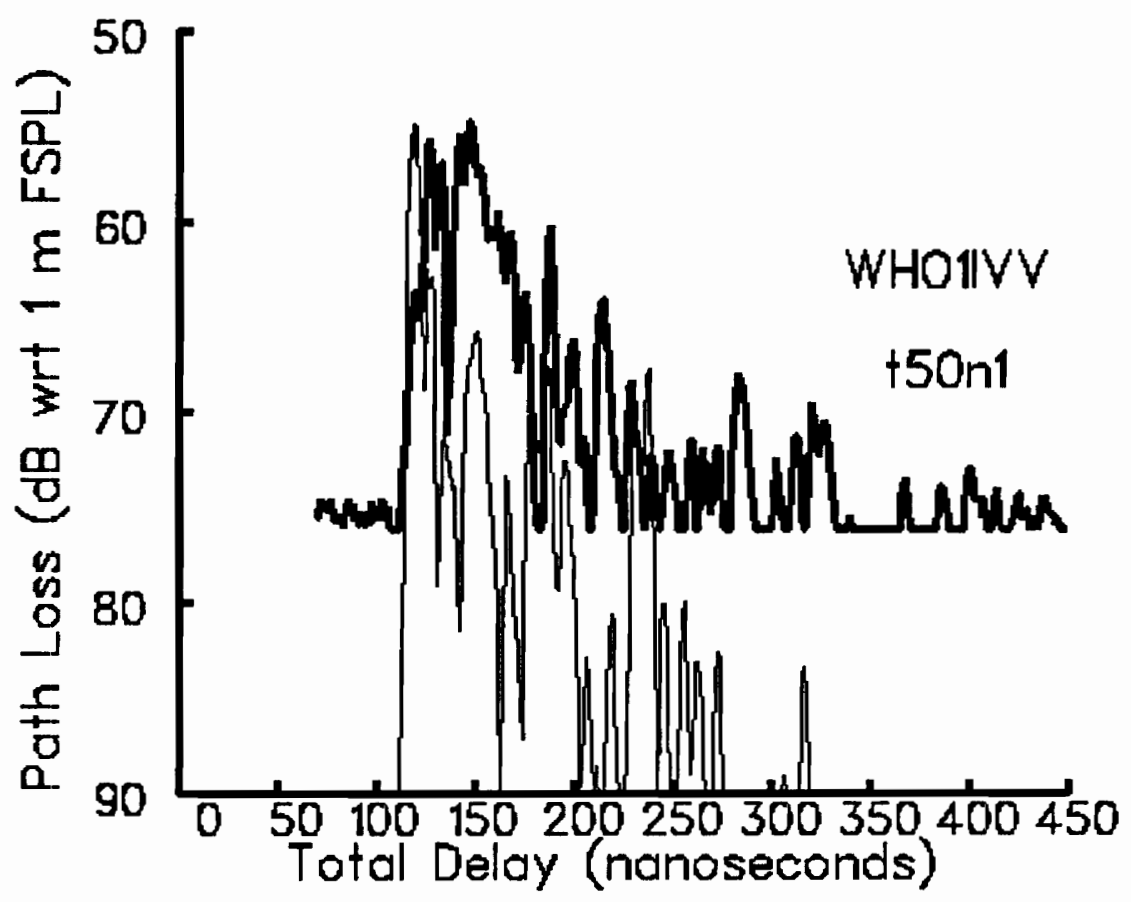



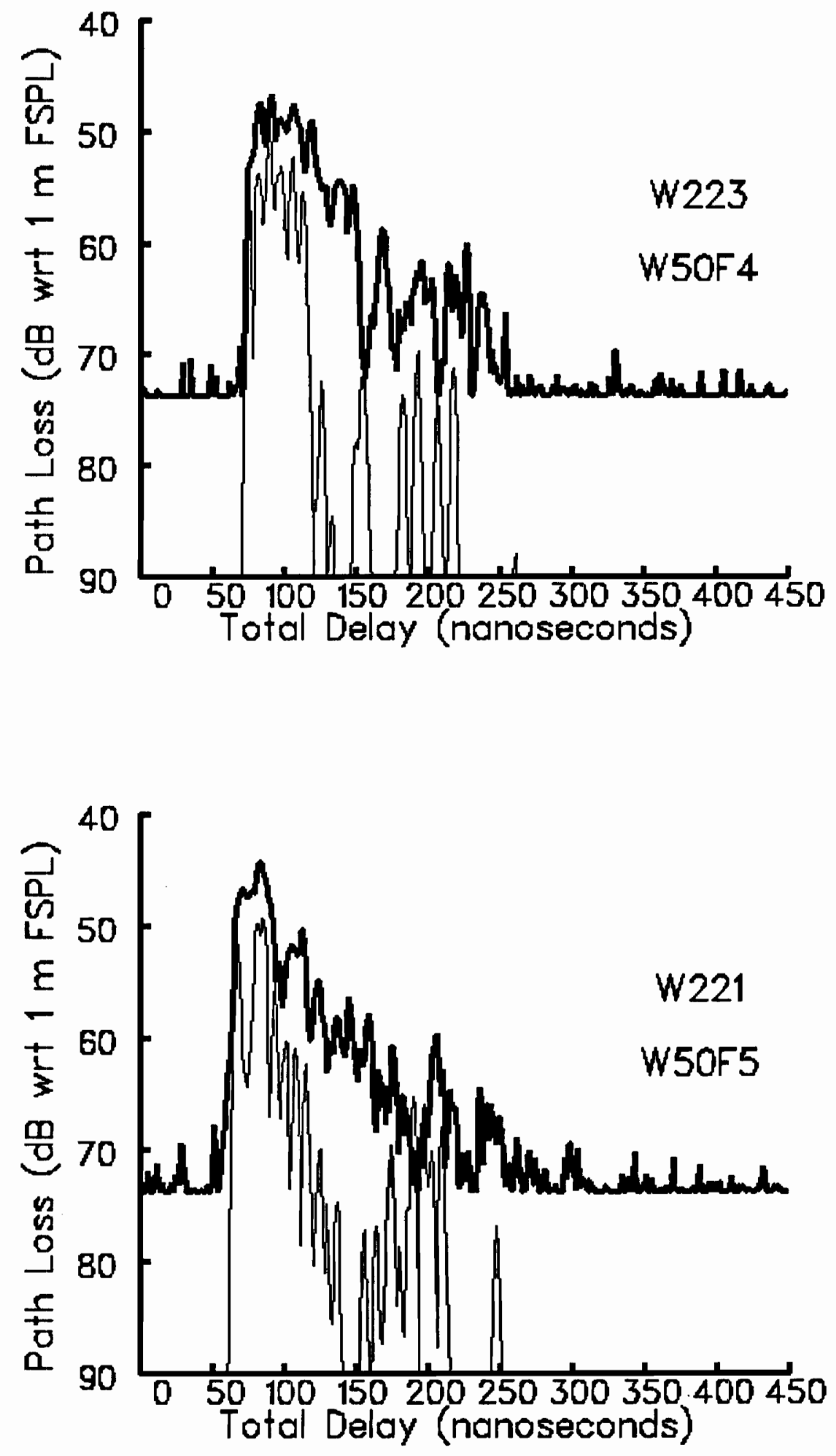

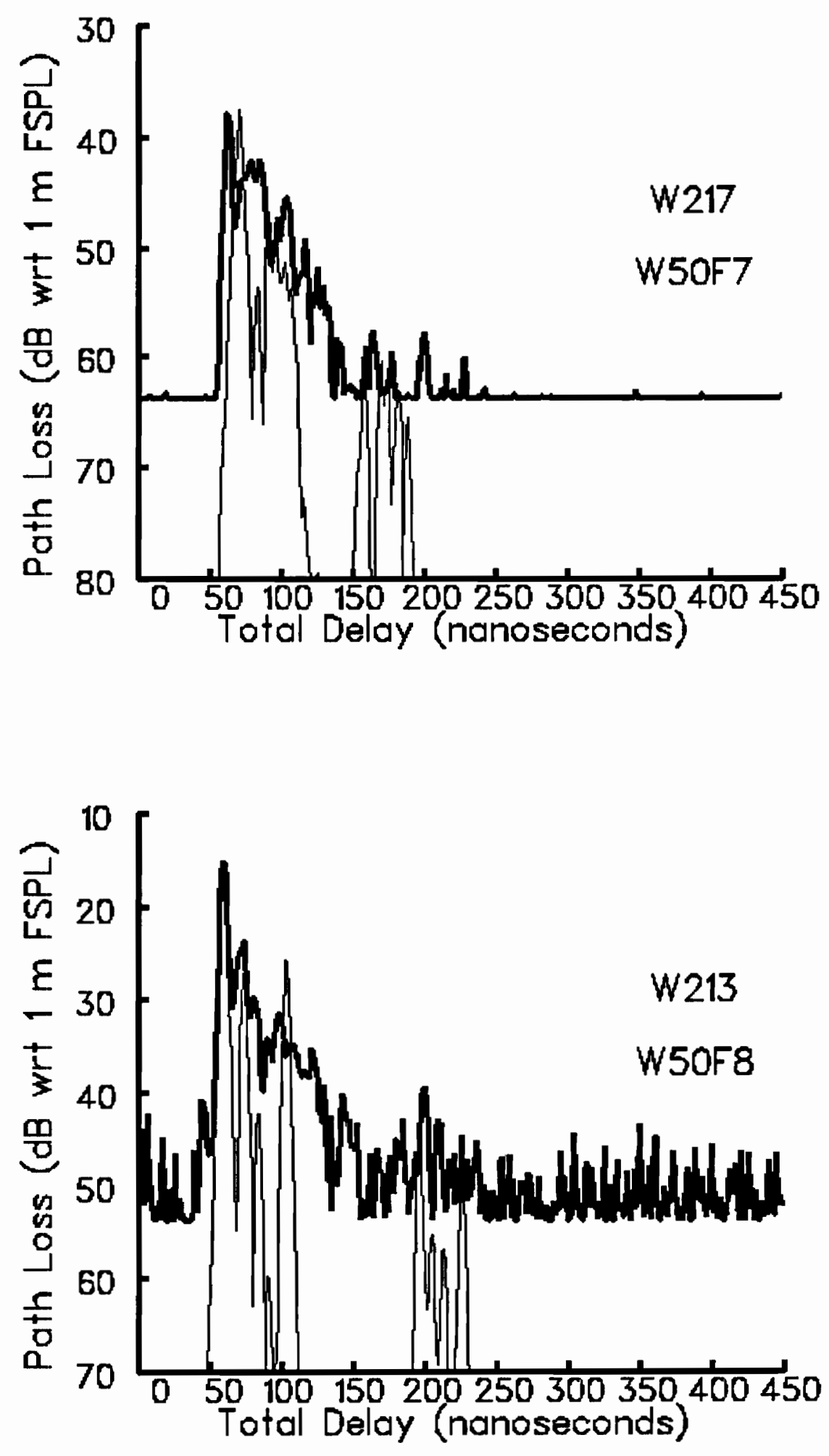

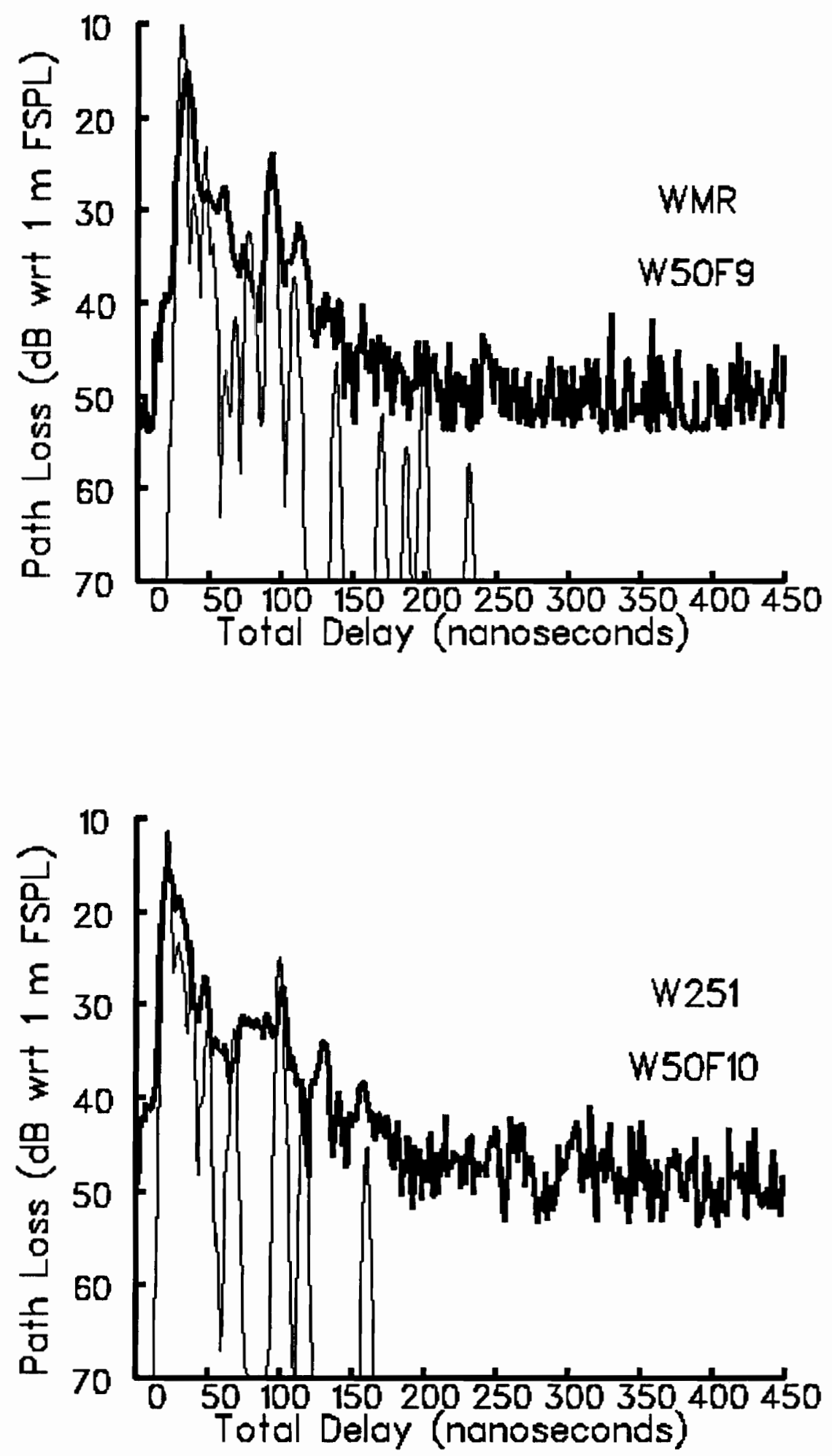

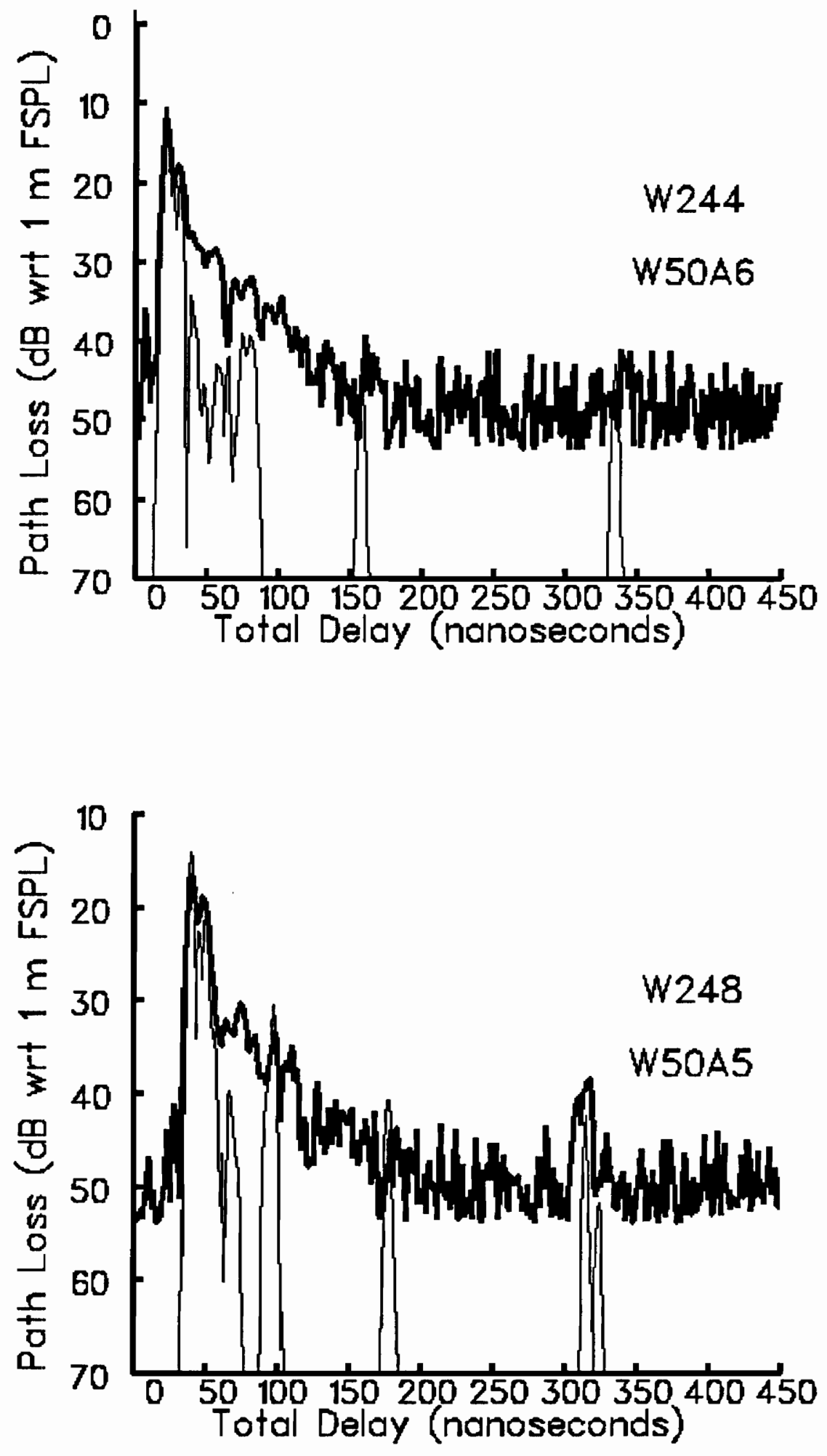

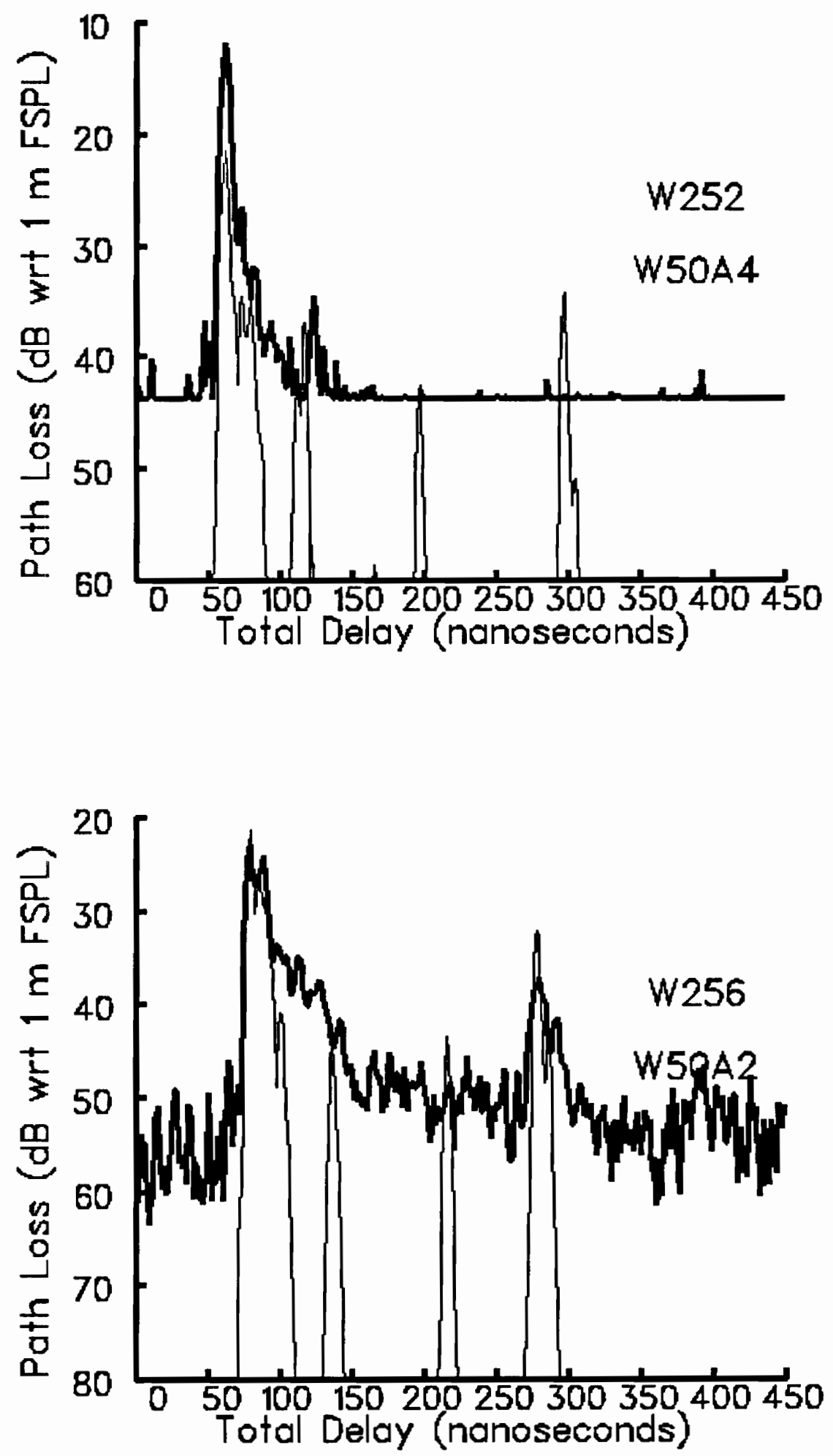

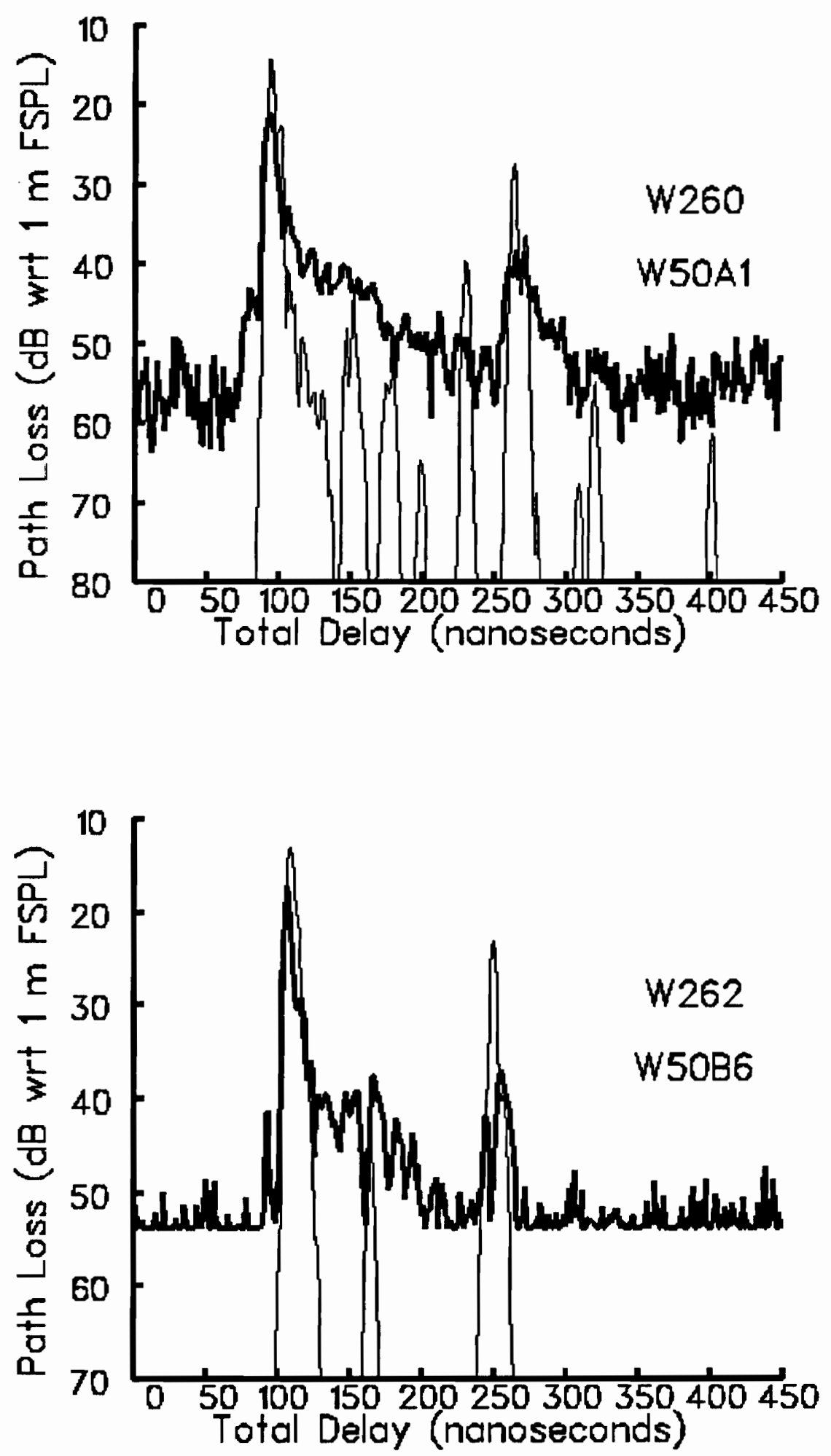

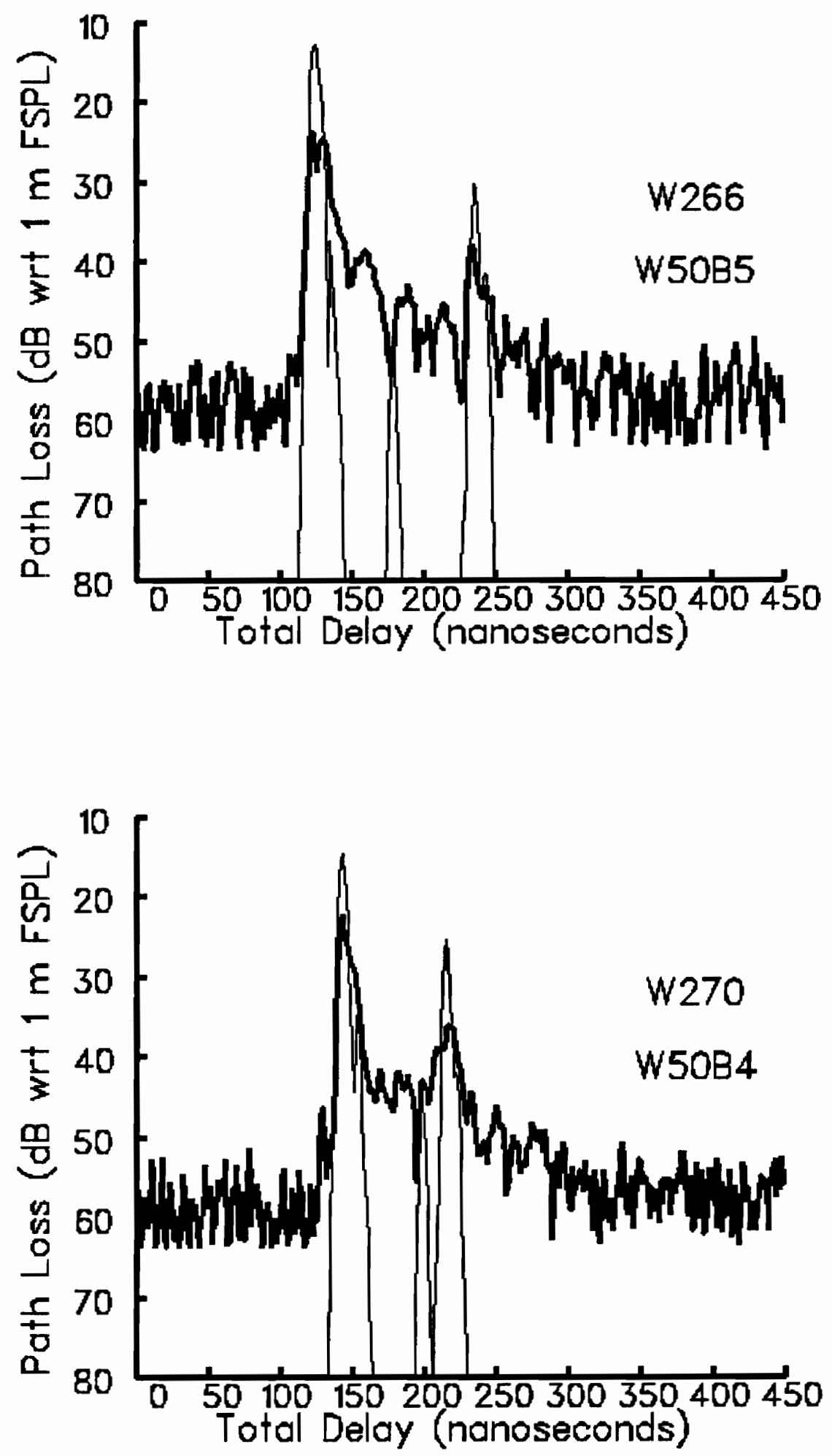

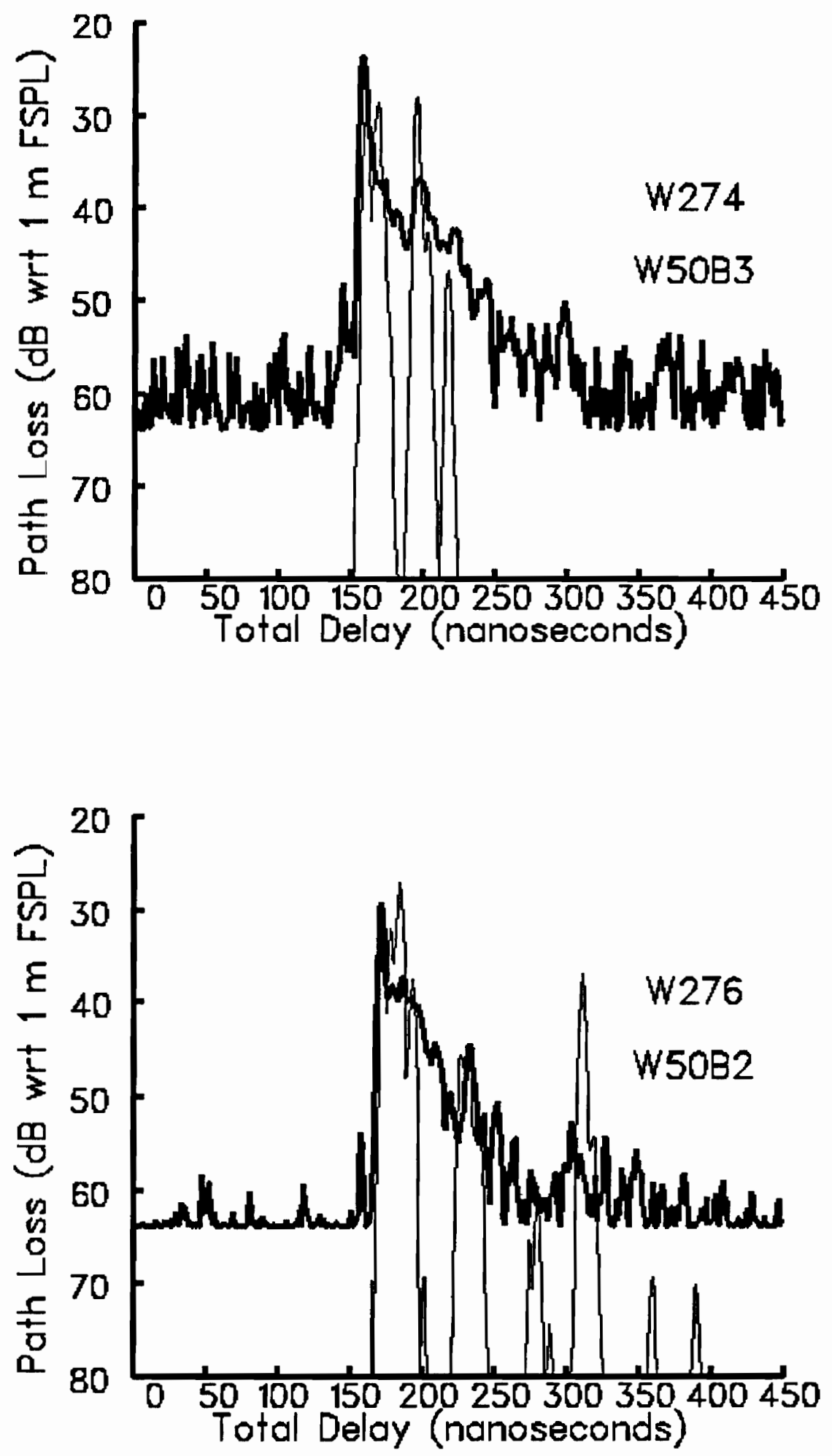

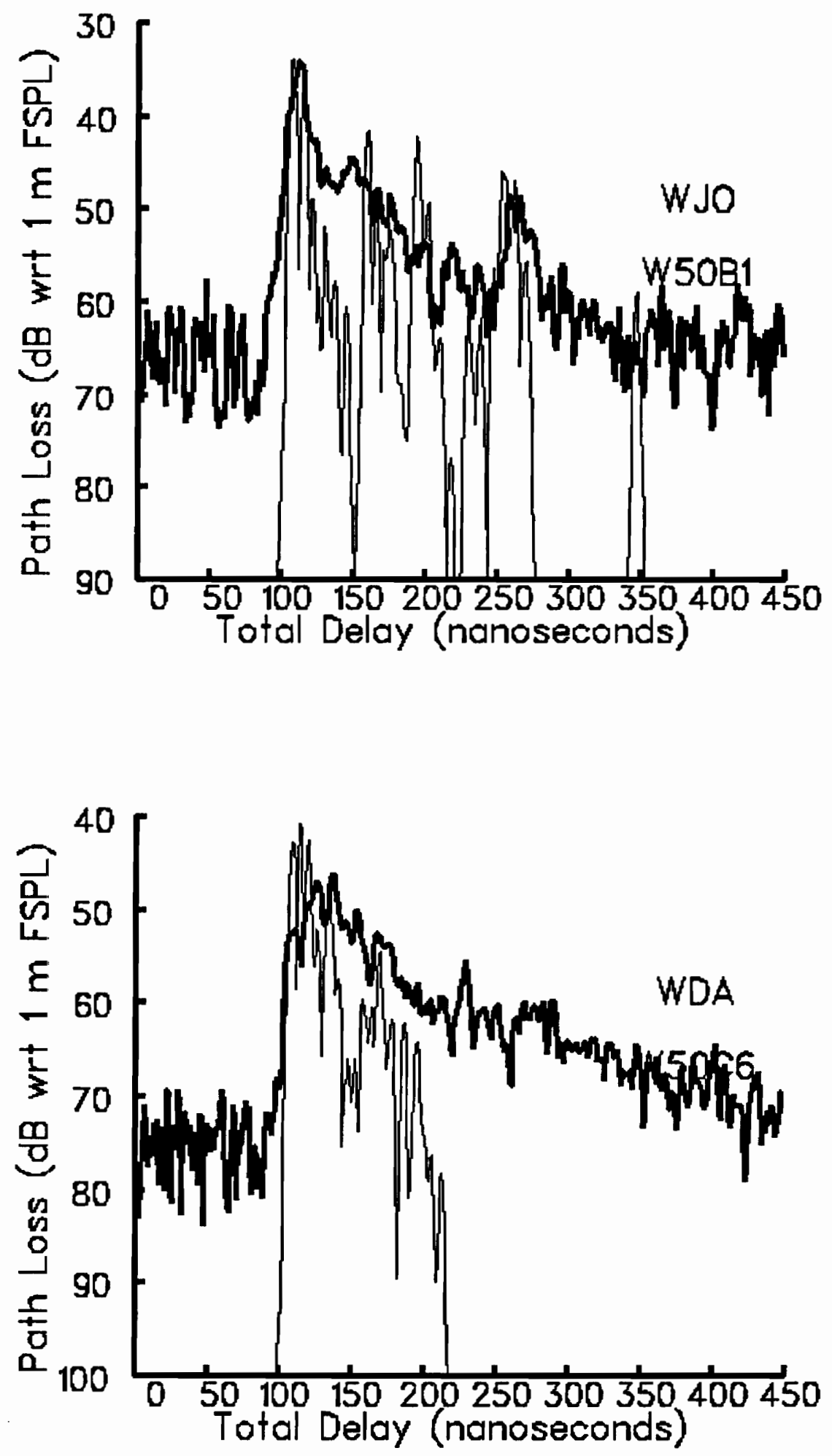

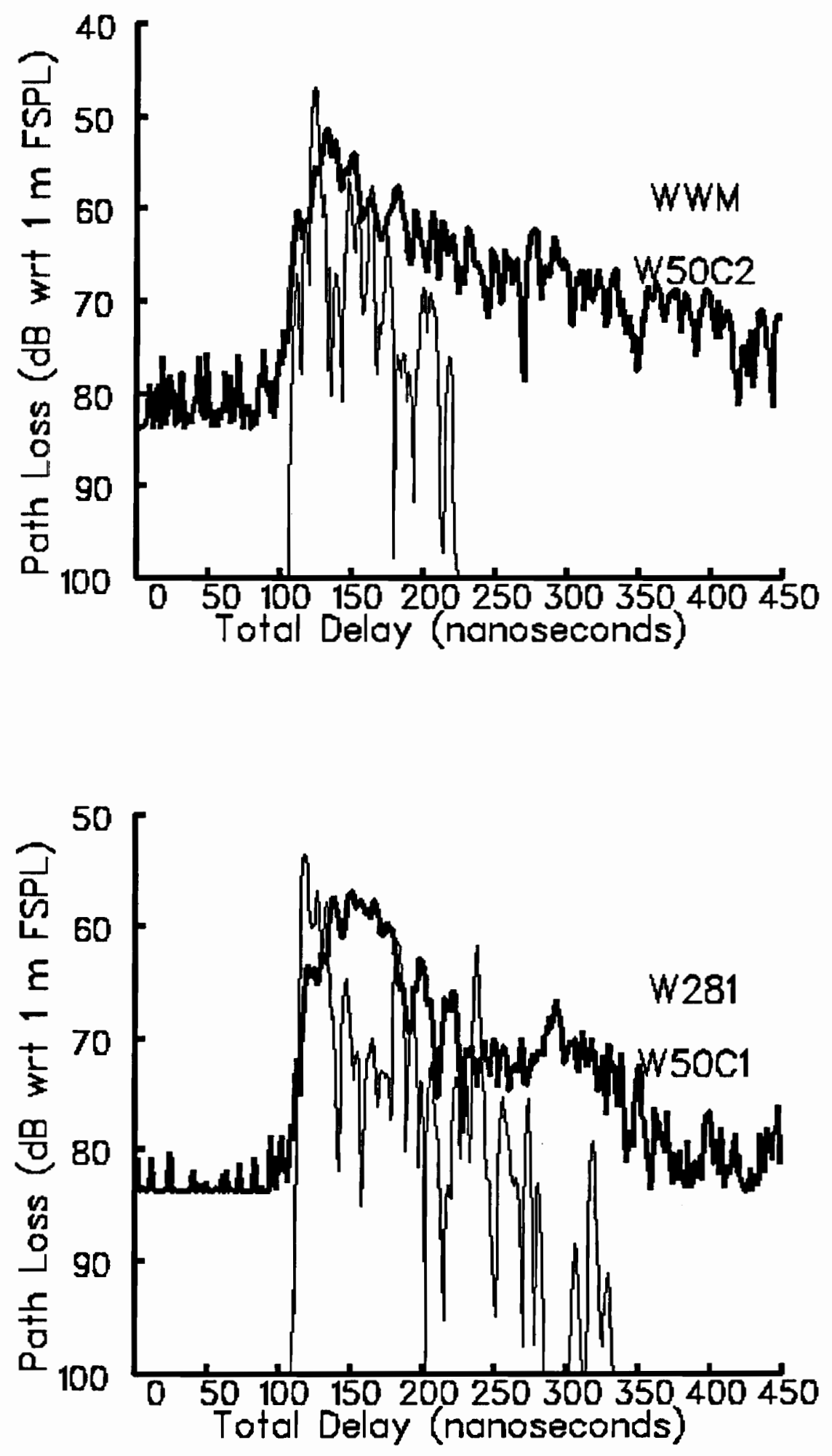


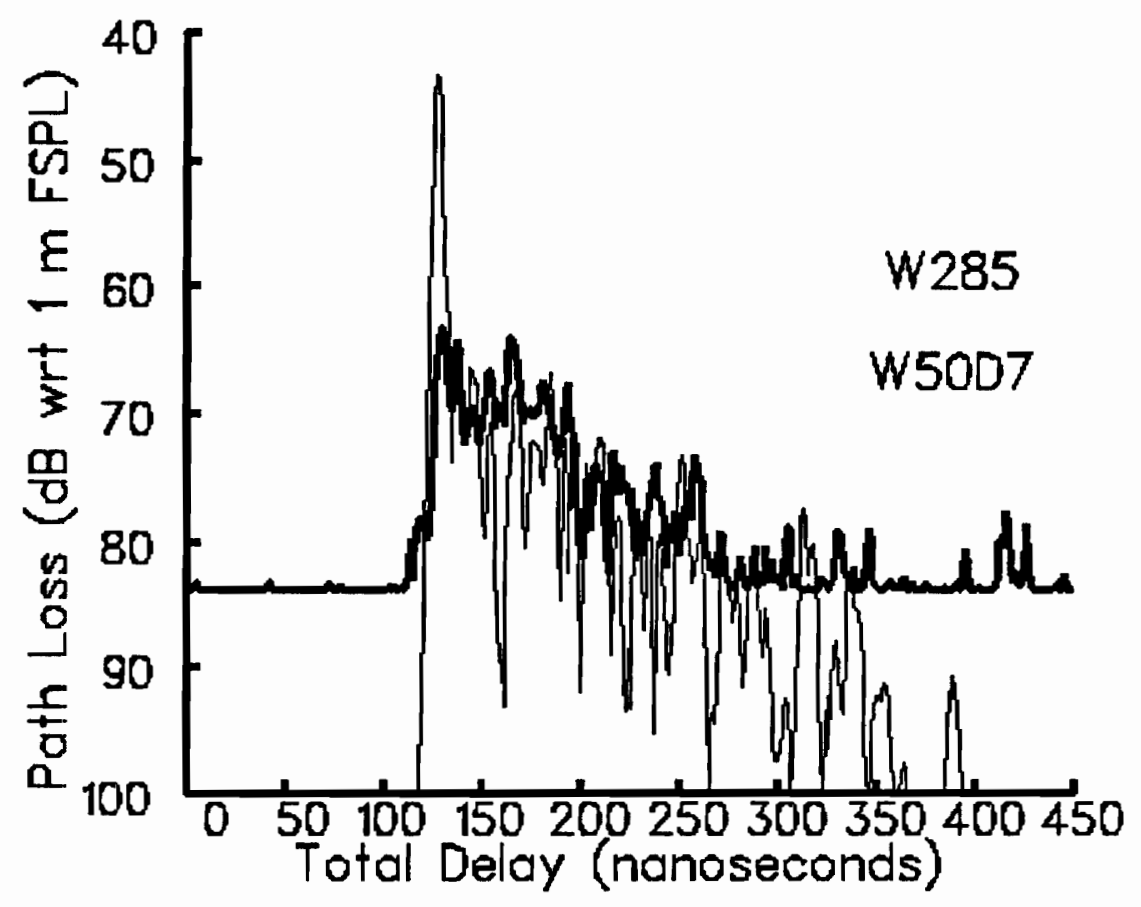



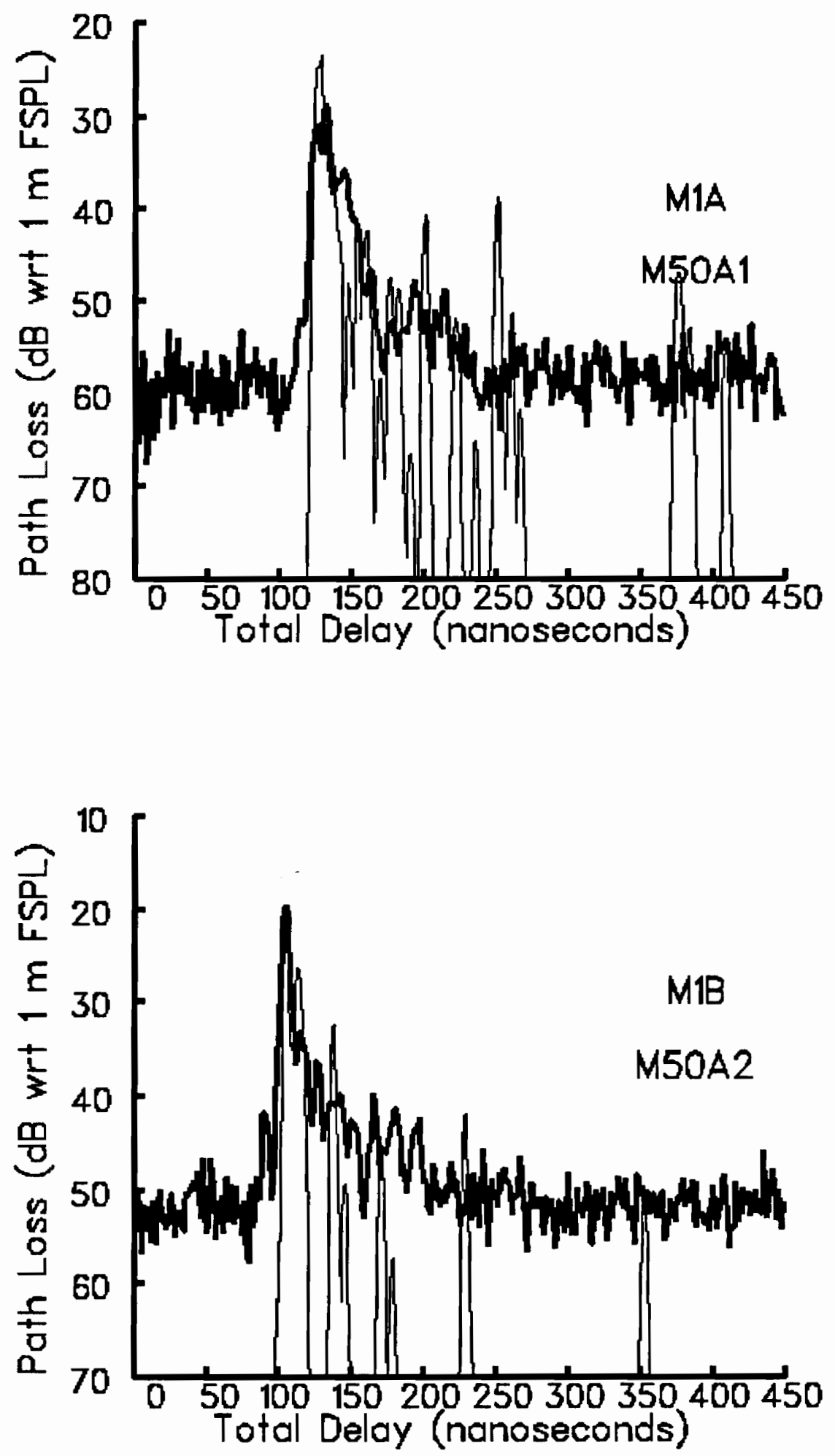

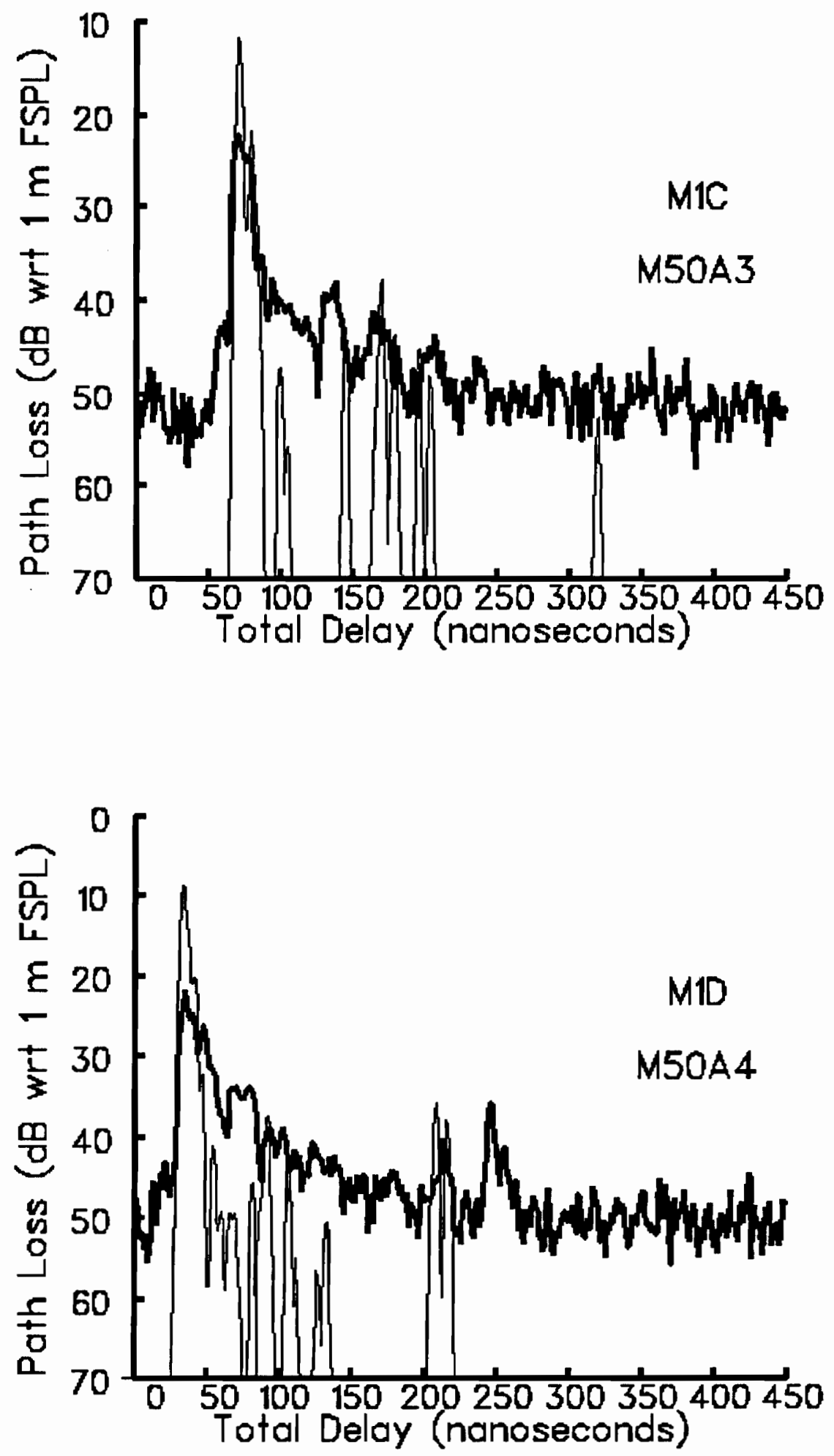

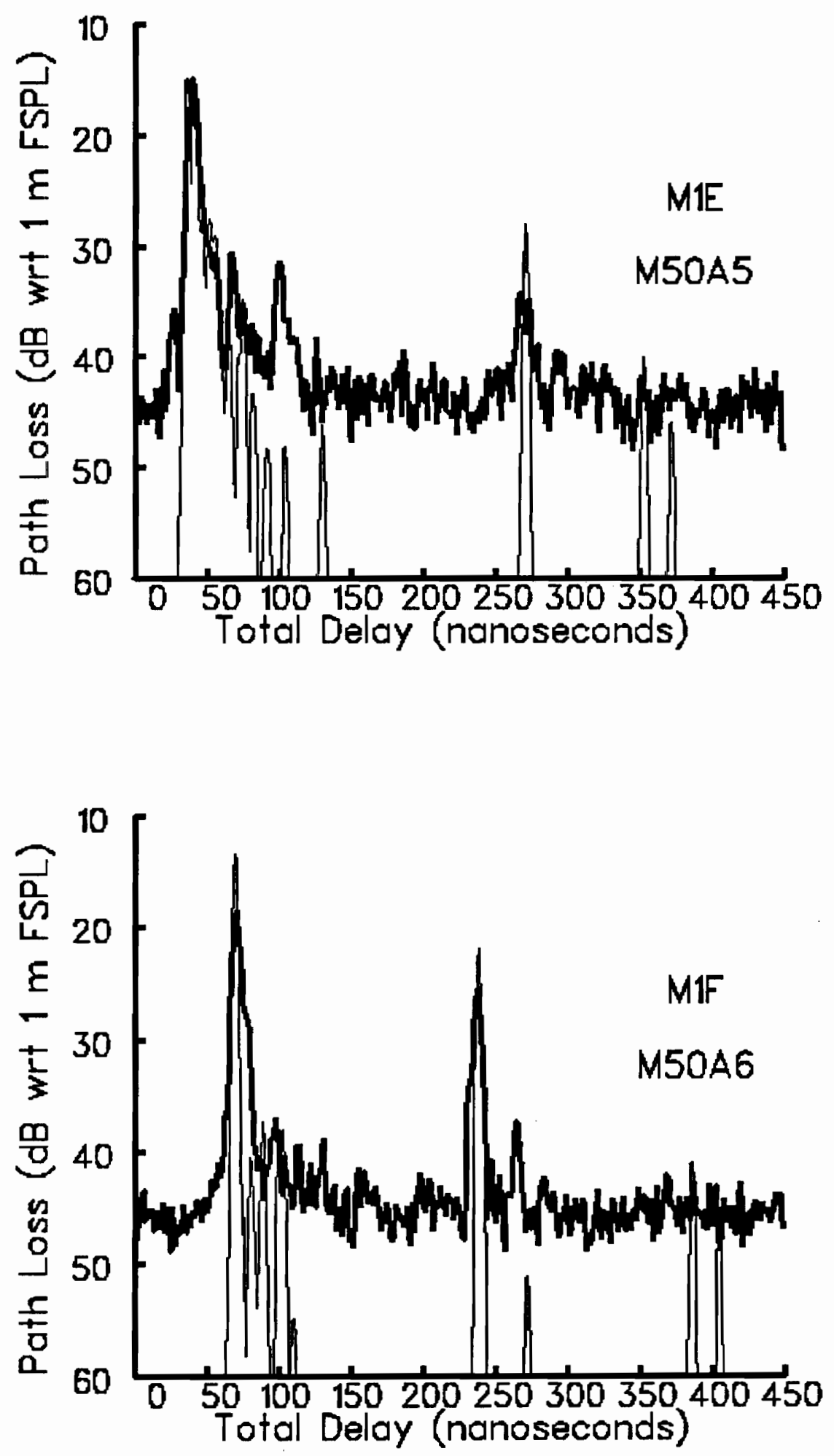

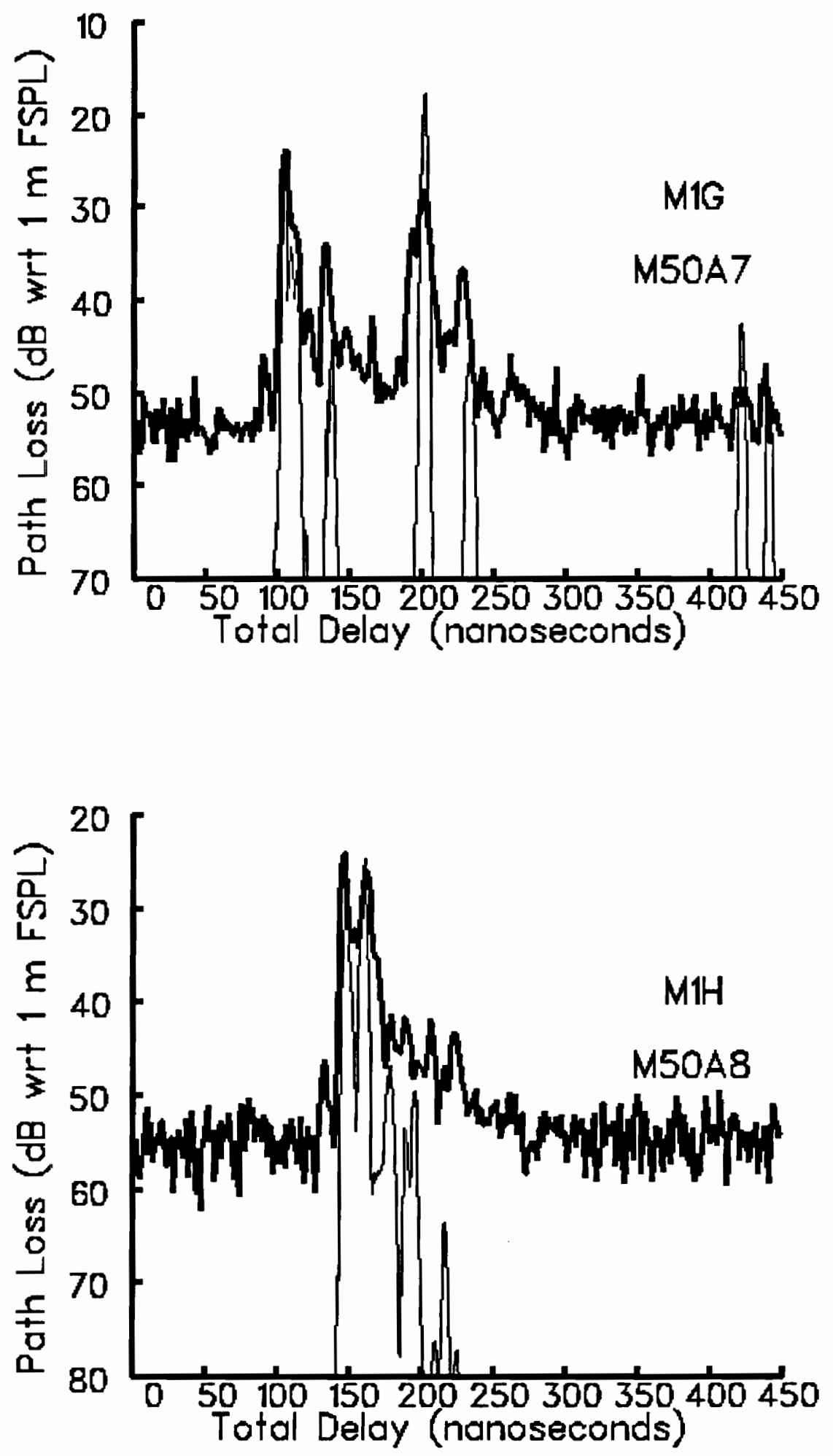

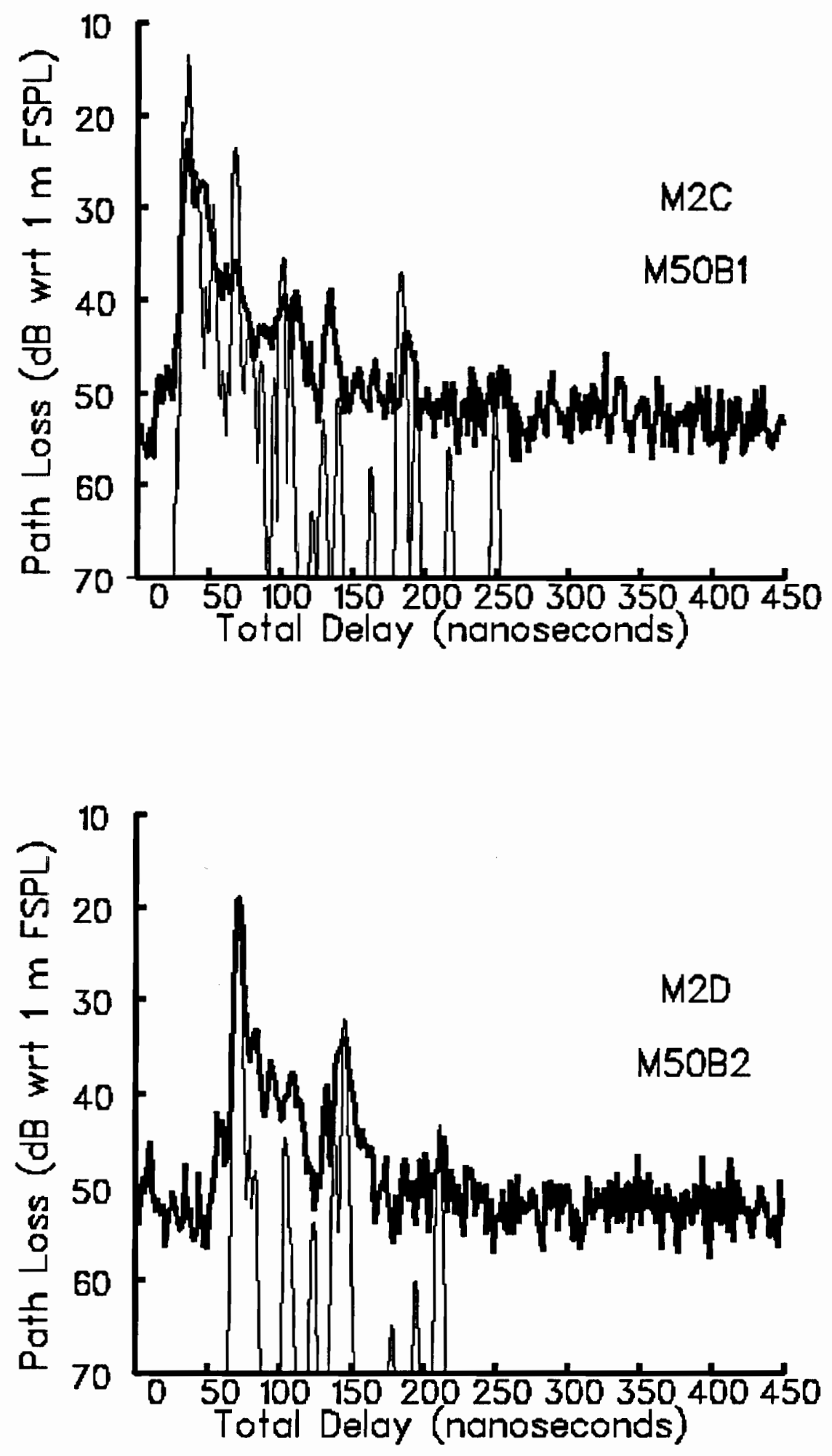

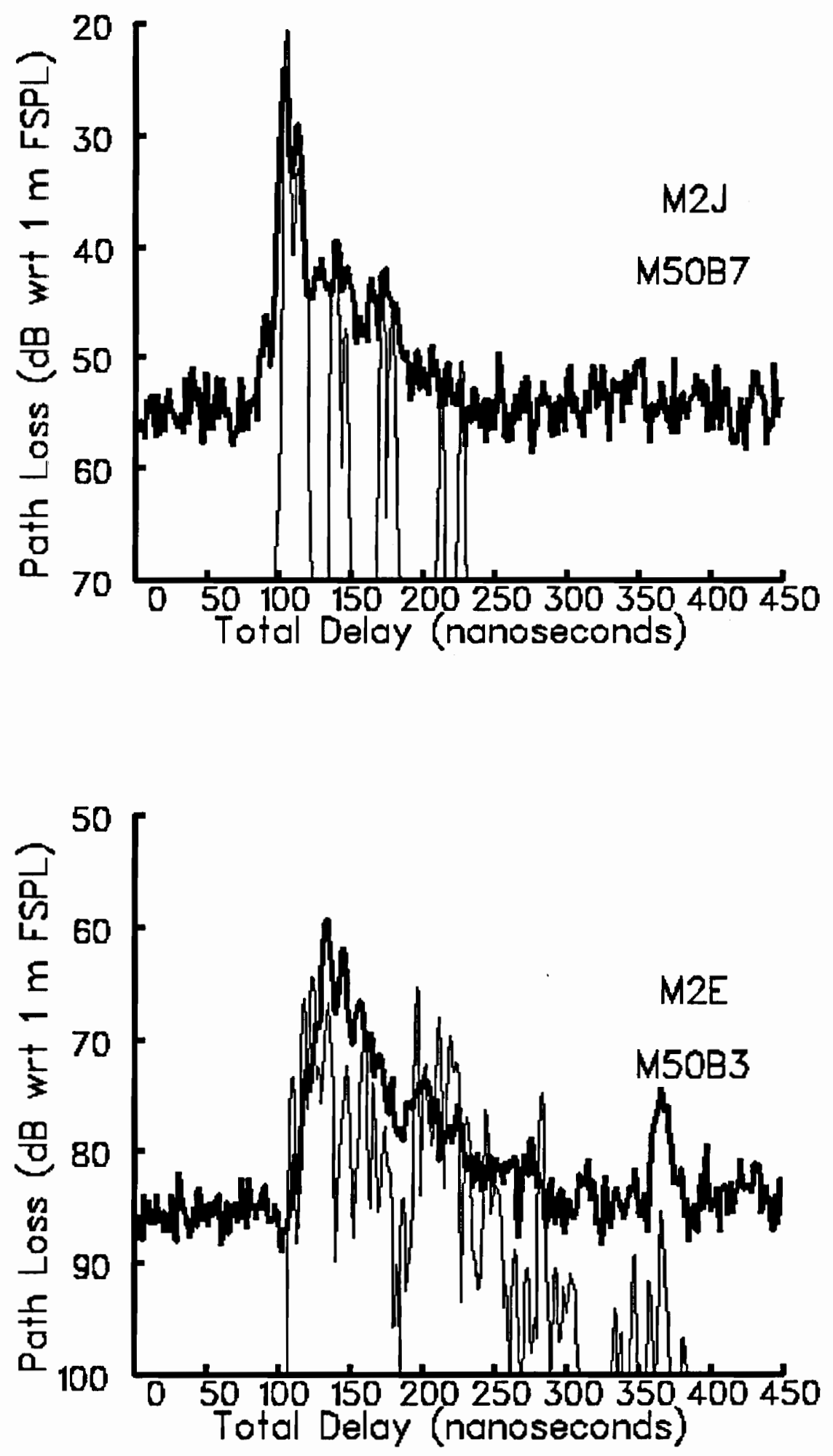

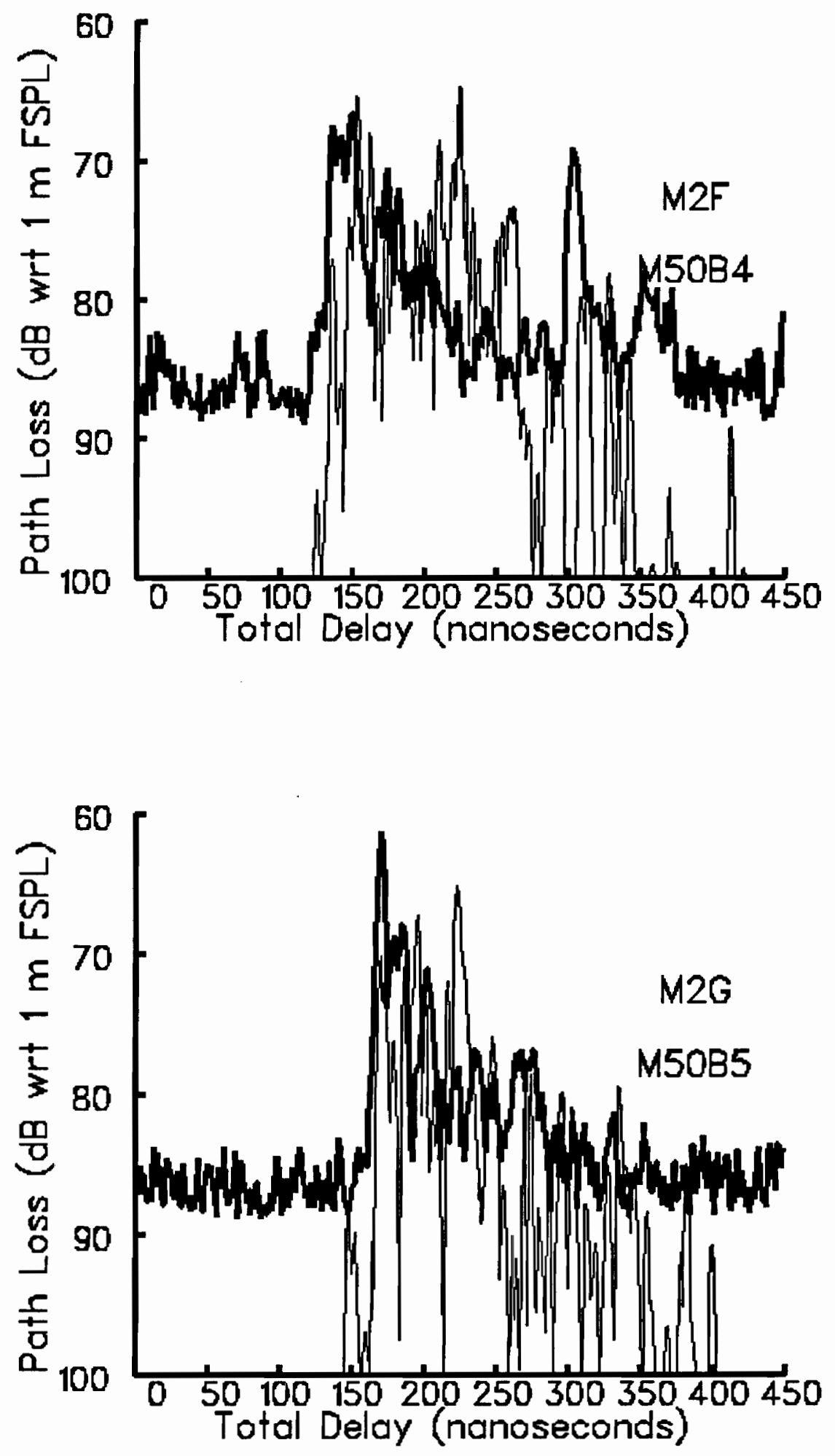


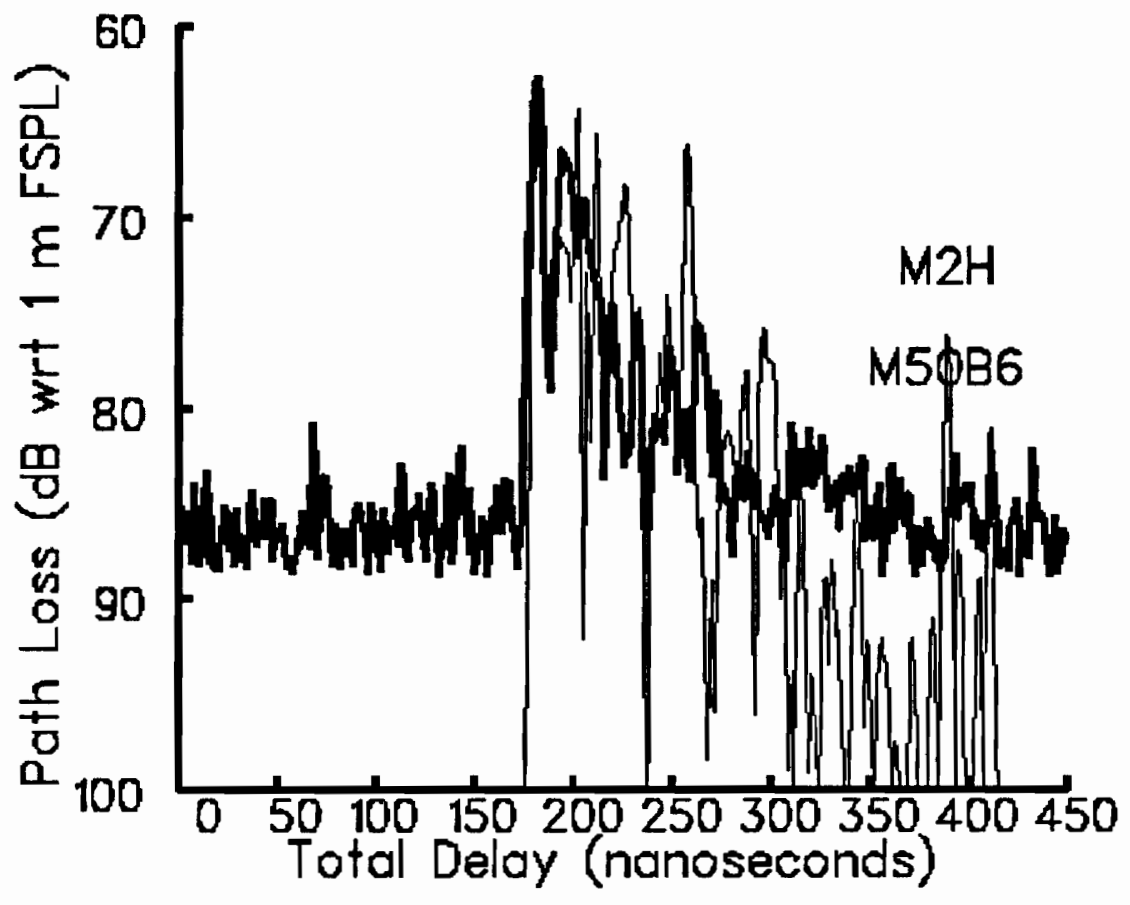




\section{Vita}

Scott Yates Seidel was born on March 2, 1966, in Falls Church, VA. He received the B.S. and the M.S. degrees in Electrical Engineering from Virginia Polytechnic Institute and State University, Blacksburg, VA, in 1988 and 1989, respectively. While an undergraduate student, he worked summers at Bendix Field Engineering, Columbia, MD. In 1989, he was a founding student member of the Mobile and Portable Radio Research Group at Virginia Tech where his research focused on propagation measurements, modeling, and prediction. During this time, Mr. Seidel was a National Science Foundation Graduate Research Fellow. In March, 1993, he begins employment at Bell Communications Research in Red Bank, NJ.

Mr. Seidel is a member of the IEEE and the Eta Kappa Nu, Tau Beta Pi, and Phi Kappa Phi honor societies.

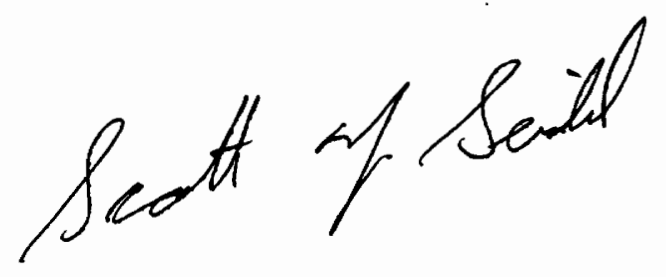

